Wall-bounded turbulence with streamwise curvature

Pieter Berghout 


\section{Graduation committee:}

Prof. Dr. J. Lynn Herek (chair)

Prof. Dr. rer. nat. D. Lohse (supervisor)

Prof. Dr. R. Verzicco (supervisor)

Dr. Ir. R. J. A. M. Stevens (co-supervisor)

Prof. Dr. Ir. H. Hoeijmakers

Prof. Dr. W. Briels

Prof. Dr. Ir. W. van Saarloos

Prof. Dr. Ir. B. Jan Boersma

Dr. D. Chung
Universiteit Twente

Universiteit Twente University of Rome Tor Vergata

Universiteit Twente

Universiteit Twente

Universiteit Twente

Universiteit Leiden

Technische Universiteit Delft

University of Melbourne

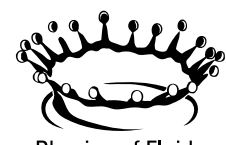

Physics of Fluids
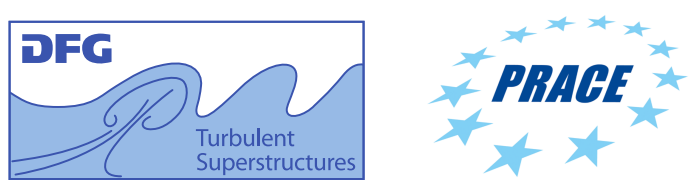

The work in this thesis was carried out at the Physics of Fluids group of the Faculty of Science and Technology of the University of Twente. This thesis was financially supported by the Priority Programme SPP 1881 Turbulent Superstructures of the Deutsche Forschungsgemeinschaft. Numerical simulations were carried out with various grants of the Partnership for Advanced Computing in Europe.

Dutch title:

Turbulente stromingen langs een gekromde wand

Publisher:

Pieter Berghout, Physics of Fluids, University of Twente, P.O. Box 217, 7500 AE Enschede, The Netherlands

Copyright (c) 2021. All rights reserved.

No part of this work may be reproduced or transmitted for commercial purposes, in any form or by any means, electronic or mechanical, including photocopying and recording, or by any information storage or retrieval system, except as expressly permitted by the publisher.

ISBN: 978-90-365-5101-4

DOI: $10.3990 / 1.9789036551014$ 


\title{
WALL-BOUNDED TURBULENCE WITH STREAMWISE CURVATURE
}

\section{DISSERTATION}

\author{
to obtain \\ the degree of doctor at the University of Twente, \\ on the authority of the rector magnificus, \\ Prof. Dr. Ir. A. Veldkamp, \\ on account of the decision of the Doctorate Board, \\ to be publicly defended \\ on Friday the $15^{\text {th }}$ of January 2021 at $16: 45$ \\ by \\ Pieter Berghout \\ Born on the $31^{\text {st }}$ of January 1992 \\ in Gouda, The Netherlands
}


This dissertation has been approved by the promotors:

Prof. Dr. rer. nat. D. Lohse

Prof. Dr. R. Verzicco

and the copromotor:

Dr. Ir. R. J. A. M. Stevens 
To my father 



\section{Contents}

1 Direct numerical simulations of Taylor-Couette turbulence: the effects of sand grain roughness 9

1.1 Introduction . . . . . . . . . . . . . . . . . . . . 10

1.2 Taylor-Couette flow . . . . . . . . . . . . . . . . 13

1.3 Numerical procedure . . . . . . . . . . . . . . . . 16

1.4 Results . . . . . . . . . . . . . . . . . . . . . . . . 19

1.5 Summary and conclusions . . . . . . . . . . . . 39

2 Calculation of the mean velocity profile for strongly turbulent Taylor-Couette flow 43

2.1 Introduction . . . . . . . . . . . . . . . . 44

2.2 Navier-Stokes equations for Taylor-Couette flow . . . . . . . 47

2.3 Employed datasets . . . . . . . . . . . . . . . . . . . . 49

2.4 Velocity profiles in Taylor-Couette turbulence . . . . . . . . . 50

2.5 The effects of the radius ratio $\eta \ldots \ldots \ldots \ldots$

2.6 The $\mathrm{Nu}(\mathrm{Ta})$ and $\mathrm{C}_{\mathrm{f}}\left(\mathrm{Re}_{\mathrm{i}}\right)$ relationships $\ldots \ldots \ldots \ldots . \ldots 63$

2.7 Summary and conclusions . . . . . . . . . . . . . 66

3 Characterizing the drag properties of rough surfaces with a $\begin{array}{ll}\text { Taylor-Couette setup } & 71\end{array}$

3.1 Introduction . . . . . . . . . . . . . . . . 72

3.2 Experimental setup and methods . . . . . . . . . . . . 77

3.3 Curvature effects . . . . . . . . . . . . . . . . . . 79

3.4 Mean velocity profiles of the inner cylinder BL . . . . . . . 83

3.5 The fully rough asymptote . . . . . . . . . . . 85

3.6 The equivalent sand grain roughness height . . . . . . . . 86

3.7 The constant angular momentum region . . . . . . . . . 88 
3.8 Calculation of $\mathrm{Nu}(\mathrm{Ta})$ and $\mathrm{C}_{\mathrm{f}}\left(\mathrm{Re}_{\mathrm{i}}\right) \ldots \ldots \ldots \ldots \ldots$

3.9 Summary, conclusions, and outlook . . . . . . . . . 93

4 Controlling secondary flow in Taylor-Couette turbulence $\begin{array}{ll}\text { through spanwise-varying roughness } & 95\end{array}$

4.1 Introduction . . . . . . . . . . . . . . . . . 96

4.2 Methods . . . . . . . . . . . . . . . . . . . 100

4.3 Results . . . . . . . . . . . . . . . . . . . . 109

4.4 Conclusions and outlook . . . . . . . . . . . . . . . 121

5 Direct numerical simulations of spiral Taylor-Couette $\begin{array}{ll}\text { turbulence } & 125\end{array}$

5.1 Introduction . . . . . . . . . . . . . . . 126

5.2 Taylor-Couette flow and numerical procedure . . . . . . . 130

5.3 Results and discussion . . . . . . . . . . . . . . . . . 131

5.4 Summary and conclusions . . . . . . . . . . . . . . . 142

6 The large-scale footprint in small-scale Rayleigh-Bénard $\begin{array}{ll}\text { turbulence } & 145\end{array}$

6.1 Introduction . . . . . . . . . . . . . . . . . . 146

6.2 Governing equations and parameters . . . . . . . . . . . . 149

6.3 Results . . . . . . . . . . . . . . . . . . . . . . . . 150

6.4 Concluding remarks . . . . . . . . . . . . 165

$\begin{array}{ll}\text { Conclusions } & 169\end{array}$

$\begin{array}{ll}\text { References } & 173\end{array}$

$\begin{array}{ll}\text { Summary } & 191\end{array}$

$\begin{array}{ll}\text { Samenvatting } & 193\end{array}$

$\begin{array}{ll}\text { Acknowledgements } & 197\end{array}$ 


\section{Introduction}

This thesis, which is a collection of independent research chapters, revolves around the topic of turbulent flows. Central, subject of the first four chapters, is the treatment of turbulent flows over rough and/or curved surfaces. Related topics of partly turbulent flows and scale interaction in turbulence are discussed in chapters five and six.

\section{Motivation}

The flow of fluids (dynamics of gases and liquids) can be categorized into two distinct classes; those flows that are orderly and well-behaved (laminar), and those flows that are chaotic, irregular and strongly fluctuating (turbulent) [1]. Both flows are described by the Navier-Stokes equations but occur when varying terms in the equation dominate. Physically spoken, when viscosity dominates inertia the flow is laminar, and when inertia dominates viscosity, the flow becomes turbulent.

In nature and engineering, the vast majority of flows is turbulent and laminar flows are only a rare exception. Examples of these turbulent flows and their relevance are: atmospheric flows and ocean currents (see figure 1) that play crucial roles in the climate and weather patterns around the globe, flows around air planes, boats, cars, bikes and many more, that play a decisive role in determining the drag, and convective flows in closed environments that govern the transport of tiny droplets that contain viruses. By studying turbulent flows, we aim to identify and understand those relationships and correlations that govern the flow and help us to ultimately better predict the climate (and weather), the drag losses in transport or the spreading of a virus. The necessity to progress our understanding of turbulent flows is thus evident.

In this thesis, we study turbulent flows that are bounded by a solid, rigid, wall. The wall imposes a no-slip boundary condition, meaning that the fluid velocity is zero at the wall. At some distance from the wall, the fluid will be 


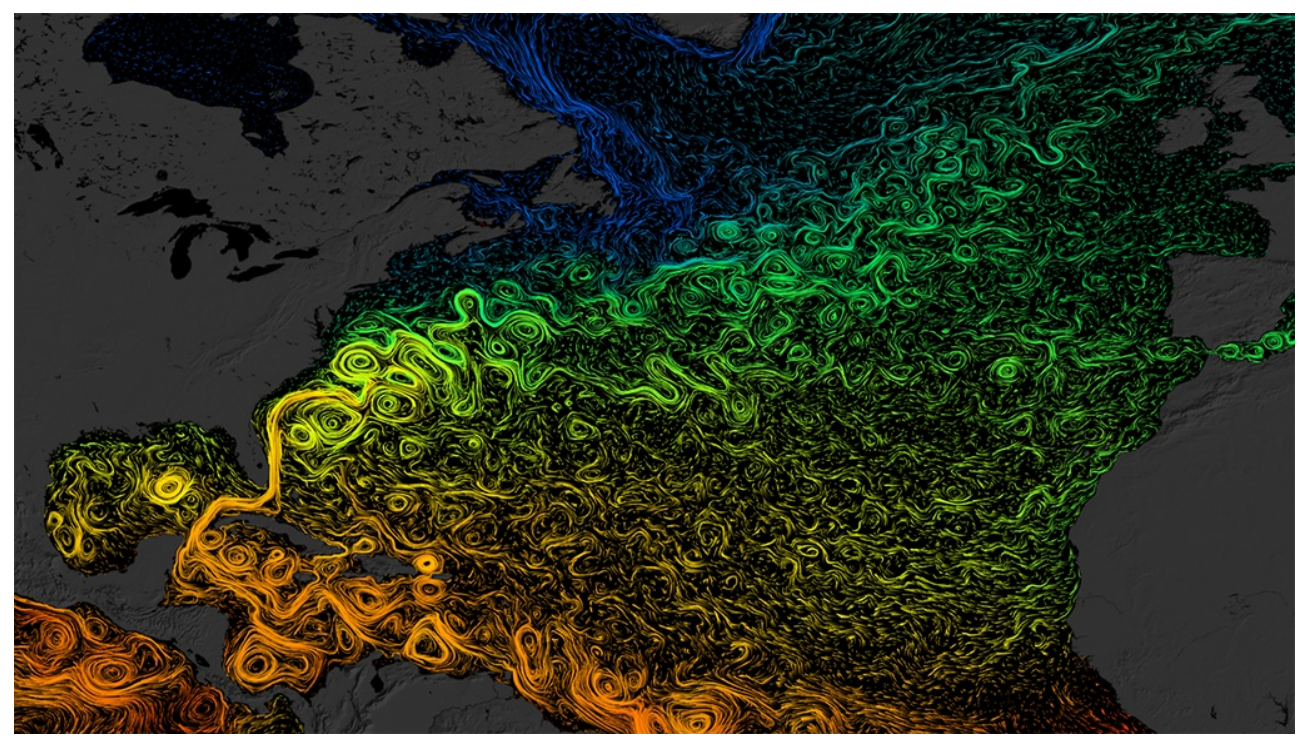

Figure 1: Visualization of very large-scale turbulent motions in the Atlantic Ocean. NASA satellite data with field measurements to present a modelled view of surface flows and gyres in the Northern Hemisphere from March 2007 to March 2008. Longer streamlines represent faster flowing fluid.

flowing freely with a certain mean velocity. Hence, there is a region directly adjacent to the wall which contains a strong gradient of the mean velocity, the so-called 'boundary layer' [2]. From a dynamical point of view, the boundary layer is arguably the most interesting region. Whereas it only occupies a small region in space, it is responsible for producing nearly all turbulent motions, and dissipating $50 \%$ of the energy that is spent in transporting fluids through pipes, or vehicles through air [3].

Researchers have spent significant time and efforts into understanding the dynamics and vortical structure of turbulent boundary layers [4]. In particular, literature focused on the idealized case of a boundary layer above a smooth, straight, wall. Given the complexity of the problem, and the many open questions that are remaining, an understandable choice. In practice however, the wall under the turbulent boundary layer is rarely smooth, and often not straight. For example, ship hulls are strongly curved near the bow, and the growth of barnacles make them very rough. Both effects have strong implications for the drag that the ship experiences and motivates our study on the dynamics of turbulent flows over curved and/or rough walls. 


\section{Roughness}

The Oxford English dictionary defines roughness as 'the quality or state of having an uneven or irregular surface'. Wikipedia mentions that 'it [the roughness] is quantified by the deviations [irregularities] in the direction of the normal vector of a real surface from its ideal form'. In the context of fluid mechanics however, whether the irregularities are large enough to bring a surface to the realm of real (i.e. rough) surfaces, or small enough to consider it an ideal (i.e. smooth) surface, depends on the fluid response. When the characteristic size of the irregularities on the surface are large enough to influence the smallest vortical motions (eddies) in the turbulent fluid flow, the surface is considered rough. For container ship hulls at cruising speed, this amounts to irregularities on the order of $10 \mu \mathrm{m}$ [5], well below the dimensions of a barnacle.

To understand the effects of roughness, and how to quantify these effects, we first zoom in on a smooth wall turbulent boundary layer. When the separation of eddie length scales in a turbulent boundary layer is large enough, there exists an inertial region where the dynamics are neither influenced by the presence of the wall, nor by the dynamics of the largest eddies. In other words, the ratio between length scales of the largest flow structure (outer length scale $\delta$ ) versus the smallest flow structure (viscous length scale $\nu / u_{\tau}$, with $\nu$ the kinematic viscosity and $u_{\tau}$ the friction velocity) has to be large, so that $R e_{\tau}=u_{\tau} d / \nu \gg 1$, for the inertial region to exist. Dimensional analysis gives that in this inertial region $\left(\nu / u_{\tau} \ll y \ll \delta\right)$, the mean velocity gradient $\frac{d U_{s}}{d y}$ is a function of the amount of momentum transfer (the wall shear stress $\tau$ ), the density of the fluid $\rho$ and the wall normal distance $y$; $\frac{d U_{s}}{d y}=K f(\tau, \rho, y)=K \tau^{1 / 2} \rho^{-1 / 2} y^{-1}$. Integration results in a logarithmic equation for the mean streamwise velocity profile,

$$
U_{s}^{+}\left(y^{+}\right)=\frac{1}{\kappa} \log y^{+}+A
$$

where $A \approx 5.0$ is the smooth wall intercept and $\kappa \approx 0.40$ is the von Kármán constant. Viscous normalization is denoted with 'plus' units, so that $U_{s}^{+}=$ $U_{s} / u_{\tau}=U_{s} / \sqrt{\tau / \rho}$ and $y^{+}=y /\left(\nu / u_{\tau}\right)=y \sqrt{\tau / \rho} / \nu$.

When the wall is rough in the fluid mechanical sense, the eddies are disturbed. In near all cases (except for riblets), this leads to extra viscous dissipation and thus a higher drag. The disturbances, often referred to as form-induced or dispersive fluctuations and stresses, are highest close to the roughness. At a sufficient distance from the wall, the dispersive fluctuations die out and the structure or dynamics of the local turbulence is identical to that of a smooth 
wall. The region in which dispersive fluctuations still play a role is called the roughness sublayer, and typically amounts to several roughness heights $k$. The notion of similarity between smooth and rough walls in the outer layer [4] is crucial for the characterization of roughness effects, and is thus often investigated in literature [6].

Since the roughness does not dynamically influence the flow when $y \gg k$, roughness only sets the boundary condition of the inertial region. For a rough wall boundary layer, we can employ a similar dimensional analysis as for a smooth wall [7] and obtain for the rough wall mean velocity $U_{r}^{+}$,

$$
U_{r}^{+}=\frac{1}{\kappa} \log (y / k)+B\left(k^{+}\right)
$$

where $B\left(k^{+}\right)$is a function of the viscous scaled roughness height and the roughness geometry. When the viscous scaled roughness height is sufficiently large $\left(k^{+} \gg 1\right)$, so that pressure forces that act on the roughness are much larger than viscous forces that act on the roughness, $B$ becomes independent of the roughness height. With equations 1 and 2 , and taking $k^{+} \gg 1$, we can define a velocity deficit $\Delta U^{+}$(or velocity shift) of the rough wall mean streamwise velocity with respect to the smooth wall streamwise velocity profile $[8,9]$, and we obtain $\Delta U^{+}=U_{s}^{+}-U_{r}^{+}=\frac{1}{\kappa} \log \left(k^{+}\right)+A-B$. In this equation (the so-called fully rough asymptote) $B$ is still a function of the roughness geometry and the key question is how this function looks like.

The convention however is to compare the drag of any rough surface to the benchmark experiments of Nikuradse, who measured the drag of a sandgrain surface in a turbulent pipe flow [10]. The sandgrain surface of Nikuradse can be considered to play the same role as a currency plays in the economy, where drag plays the role of purchasing power. In comparing to the effect of sandgrains, the fully rough asymptote is rewritten to,

$$
\Delta U^{+}=\frac{1}{\kappa} \log \left(k_{s}^{+}\right)+A-\tilde{B}
$$

where $\tilde{B} \approx 8.50$ is the Nikuradse constant and $k_{s}^{+}$is the sandgrain roughness height $k_{s}^{+}=c k^{+}$, where $c$ is a constant. For pipe flow, straightforward integration of the mean velocity profile leads to an expression for the skinfriction coefficient versus the Reynolds number (the Moody chart), which is the relevant engineering tool for predicting the drag [11].

In conclusion, in knowing $k_{s}$ of any homogeneously rough surface, we know the drag at any Reynolds number. The central question in the field of rough, wall-bounded turbulent flows is therefore: How does the value of $k_{s}$ relate to the properties of a homogeneously rough surface? 


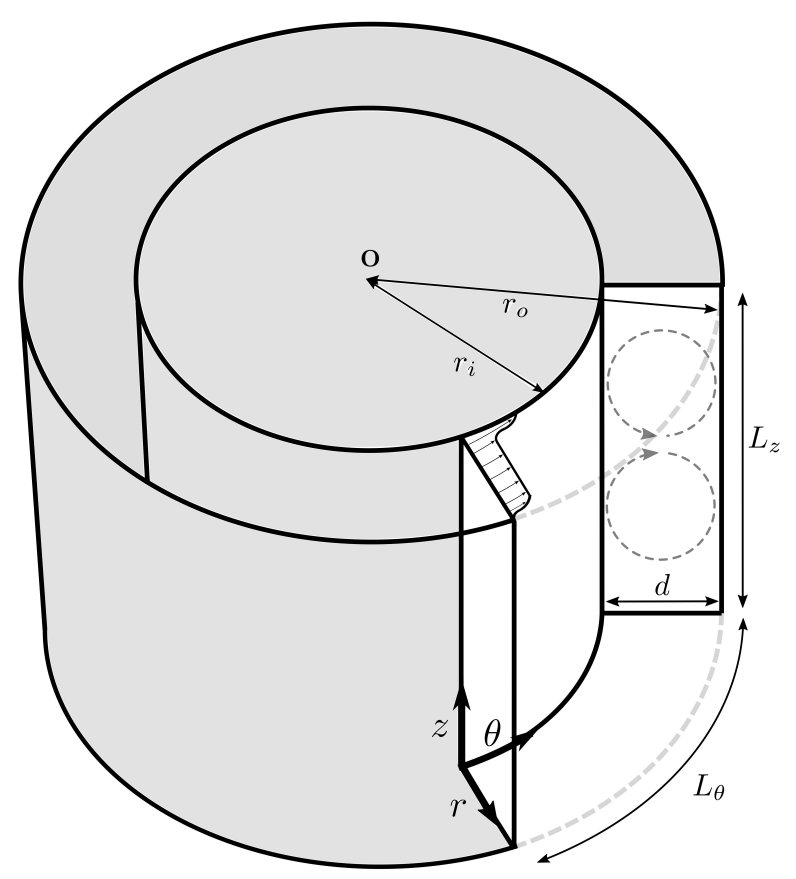

Figure 2: Schematic of TC flow including the coordinate directions $(\theta, z, r)$, inner cylinder (IC) radius $r_{i}$, outer cylinder (OC) radius $r_{o}$, gap width $d$, the spanwise (axial) extent of the flow domain $L_{z}$, and the streamwise extent of the flow domain $L_{\theta}$, which is used in DNSs that employ periodic boundary conditions in the azimuthal directions. $\eta=r_{i} / r_{o}$ is the radius ratio. The grey dashed circular arrows represent the turbulent Taylor vortices. (Reproduced in chapter 2)

\section{Taylor-Couette flow}

To investigate the effects of roughness and curvature on a turbulent boundary layer, we study one of the canonical systems of fluid mechanics, Taylor-Couette (TC) flow $[12,13]$, see figure 2. Conceptually, the system is very simple. The fluid in between two concentric and independently rotating cylinders with radii $r_{i}<r_{o}$ is driven by the shear on the rotating cylinder. Since the domain is closed, global balances can easily be derived and monitored, giving room for extensive comparison between theory, experiments and simulations. Compared to other canonical systems as pipe flow and turbulent boundary layer, the system is very small and very long spatial transients, as found in open 
systems, are bypassed by the circumferential restrictions. Most importantly for the purpose of roughness research, the TC systems allows for direct and accurate measurements of the the drag, which is much more cumbersome for e.g. turbulent boundary layers.

\section{Guide through the thesis}

The seminal experiments of Nikuradse, in which he measured the drag of turbulent flows over sand grain surfaces, serve as the reference case for the study of rough wall-bounded turbulence without streamwise curvature (e.g. pipe flow). In chapter 1, we investigate how the effects of sand grains on wallbounded turbulence with streamwise curvature (e.g. Taylor-Couette flow) compare to the experiments of Nikuradse. We do this by simulating a rough surface that resembles the surface that Nikuradse used, followed by direct numerical simulations of turbulent Taylor-Couette flow at varying rotation rates. The findings in chapter 1 , and especially the differences between rough pipe flow and rough TC flow, directly lead to the hypotheses that lay basis for chapters 2 and $3^{1}$.

Turbulent flow over a smooth wall serves as the reference case to quantify the drag penalty induced by a rough wall. However, in chapter 1 we find that even the mean velocity profiles of smooth TC flow and pipe flow differ, and prohibit a comparison of drag between the 2 systems a priori. Hence, in chapter 2, we shift our focus to smooth TC turbulence, and in particular, we aim to understand the shape of the mean velocity profile. Decisive in our efforts is the theory laid out by Peter Bradshaw in a 1969 paper on curvature effects [14]. With the help of experimental and numerical data gathered by foregoing research in the Physics of Fluids group, we derive an equation that satisfactorily describes the shape of the mean velocity profile in smooth wall TC turbulence.

We then move back to study the effects of roughness in chapter 3. This time however we carry out experiments at very high rotation rates, in a collaborative study with experimentalist from Munich and the Physics of fluids group. In the experiments, as compared to numerical simulations, we are able to achieve a far higher length scale separation between the smallest structures in the turbulent flow field, the roughness height and the gap width of the apparatus. This scale separation is mandatory to apply many of the basic modelling assumptions of turbulent boundary layers. With the newly derived mean velocity profile for

\footnotetext{
${ }^{1}$ Chapters 2 and 3 are at the heart of this thesis.
} 
smooth wall Taylor-Couette turbulence in chapter 2 and the measurements of the velocity statistics of turbulent Taylor-Couette flow with a rough wall, we are now able to much better understand the effects of roughness on TC turbulence and how it compares to pipe flow. Finally, following the recent theory of Cheng et al. [15], we calculate the torque from the mean velocity profiles.

The remaining 3 chapters $(4,5$ and 6$)$ contain less mutual coherence and no chronological order. Rather, they link to the overarching themes of roughness, Taylor-Couette turbulence or even turbulence in general. Chapter 4 deals with 'unusual' roughness. Unusual, non-homogeneous, roughness means in this context that the roughness contains a characteristic length scale that is not smaller than the gap width, but is of the same order. This leads to the invalidation of a classic modelling assumptions (outer layer similarity), and consequently adds majorly to the complexity of the problem.

In chapter 5 we shift the focus again to Taylor-Couette flow with smooth walls, and study the emergence and dynamics of very large structures at the onset of turbulence (or better, the onset of laminarization). These structures, already observed by D. Coles (1965) [16], manifest themselves as large turbulent spirals that slowly disappear when the cylinders rotate slower and slower. Prigent et al. (2000) [17] found that the formation of these spirals fits the phenomenology of the Complex Ginzburg-Landau model and this suggests that they are a finite wavelength instability. In our simulations, we show that this description holds when curvature effects do play a role.

Finally, we study Rayleigh-Bénard flow, the flow between a heated and cooled plate, in chapter 6 . In particular we focus on the frequency and amplitude modulation of small-scale turbulence by the large-scale convection role. 


\section{Chapter 1}

\section{Direct numerical simulations of Taylor-Couette turbulence: the effects of sand grain roughness}

Progress in roughness research, mapping any given roughness geometry to its fluid dynamic behaviour, has been hampered by the lack of accurate and direct measurements of skin-friction drag, especially in open systems. The TaylorCouette (TC) system has the benefit of being a closed system, but its potential for characterizing irregular, realistic, 3-D roughness has not been previously considered in depth. Here, we present direct numerical simulations (DNSs) of TC turbulence with sand grain roughness mounted on the inner cylinder. The model proposed by Scotti [18] has been modified to simulate a random rough surface of monodisperse sand grains. Taylor numbers range from $T a=$ $1.0 \times 10^{7}$ (corresponding to $\left.R e_{\tau}=82\right)$ to $T a=1.0 \times 10^{9}\left(R e_{\tau}=635\right)$. We focus on the influence of the roughness height $k_{s}^{+}$in the transitionally rough regime, through simulations of TC with rough surfaces, ranging from $k_{s}^{+}=5$ up to $k_{s}^{+}=92$. We find that the downwards shift of the logarithmic layer, due to transitionally rough sand grains exhibits remarkably similar behavior to that of the Nikuradse [10] data of sand grain roughness in pipe flow, regardless of the Taylor number dependent constants of the logarithmic layer. Furthermore, we find that the dynamical effects of the sand grains are contained to the roughness sublayer $h_{r}$ with $h_{r}=2.78 k_{s}$.

Published as: P. Berghout, X. Zhu, D. Chung, R. Verzicco, R.J.A.M. Stevens, and D. Lohse, Direct numerical simulations of Taylor-Couette turbulence: the effects of sand grain roughness, J. Fluid Mech. 873, 260-286 (2019). 


\subsection{Introduction}

Many turbulent flows in nature and industry are bounded by rough boundaries. Examples are the surface of planet earth with respect to geophysical flows or fouling on ship hulls with respect to open waters. Such rough boundaries strongly influence the total drag, with often adverse consequences in the form of higher transport costs. Therefore, it becomes of paramount importance to understand the physics behind such changes in drag, ultimately leading to better informed management of the problem. One key recurring question concerns the influence of the roughness topology on the drag coefficient.

Seminal work by [10] investigated the influence of the height of closely packed, monodisperse, sand grains in pipe flow. This work has become one of the pillars in the field. Later, a vast amount of research was carried out to study the influence of roughness on the canonical systems of turbulence - pipe, channel, and boundary layer flows - aiming for a better understanding of the roughness effects on turbulent flows [19-22]; also see [6], and [23] for comprehensive reviews.

Next to pipe flow, Taylor-Couette (TC) flow - the flow between two coaxial, independently rotating cylinders - is another canonical system in turbulence [13]. Closely related to its 'twin' of Rayleigh-Bénard (RB) turbulence [24,25], it serves as an ideal system to study the interaction between boundary layer and bulk flow. Very long spatial transients, as found in open systems, are bypassed by the circumferential restrictions. Since the domain is closed, global balances can easily be derived and monitored, giving room for extensive comparison between theory, experiments and simulations. Further, the streamwise curvature effects find many applications in industry. For these reasons, we set out to investigate the effects of roughness on the turbulent fluid flow in the TC system.

Over the last century, much work has been carried out with the aim of understanding the effect of the roughness topology on fluid flow. One of the consequences of roughness is the change of the wall drag, which can be expressed as a shift of the mean streamwise velocity profile $\Delta u^{+} \equiv\left(u_{s}-u_{r}\right) / u_{\tau}$, where $\Delta u^{+}$is known as the Hama roughness function [8] and $u_{s}, u_{r}$ are the smooth-wall and the rough-wall mean streamwise velocities, respectively. [9] and [8] observed that roughness effects are confined to the inner region of the boundary layer. This idea was postulated by [4], who referred to it as Reynolds number similarity. The hypothesis states that outside the roughness sublayer, the structure of the flow is independent of the wall roughness, except for the 
role of the wall in setting the wall stress $\tau_{w}$. The hypothesis, now known as Townsend's outer layer similarity, has found strong support over time [7,26]. The logarithmic region is thus dynamically not influenced by the roughness and the mean streamwise velocity profile $u(y)$ there becomes [11]

$$
u^{+}\left(y^{+}\right)=\frac{1}{\kappa} \log y^{+}+A-\Delta u^{+}
$$

As usual, the superscript ' + ' indicates a scaling in viscous units (i.e. length $y^{+}=y u_{\tau} / \nu$ and velocity $\left.u^{+}=u / u_{\tau}\right)$ and $u_{\tau}$ is the friction velocity, $u_{\tau}=$ $\sqrt{\tau_{w} / \rho}$ with $\tau_{w}$ being the total stress at the wall, and $\rho$ the fluid density. We calculate $\tau_{w}$ at the outer wall, which is smooth. Because the torque is the same on both cylinders, we can calculate the wall shear stress on the inner cylinder by $\tau_{w, i}=\tau_{w, o} / \eta^{2}$, where $\eta$ is the radius ratio. Note that in this representation, the skin-friction coefficient $C_{f}$ is related to the friction velocity by $C_{f}=2\left(u_{\tau} / U_{0}\right)^{2}$, where $U_{0}$ is the centerline velocity [11]. It has been found that for TC turbulence $\kappa$ and $A$ are not constant anymore, but are functions of the inner cylinder Reynolds number $R e_{i}$ at least until $R e_{i}=10^{6}$ [27]. Therefore, for TC we here suggest the generalization;

$$
u^{+}\left(y^{+}\right)=f_{1}\left(R e_{i}\right) \log \left(y^{+}\right)+f_{2}\left(R e_{i}\right)-\Delta u^{+}
$$

with $f_{1}\left(R e_{i}\right)$ and $f_{2}\left(R e_{i}\right)$ being unknown functions. The questions now are: i) How does $\Delta u^{+}$depend on the parameters that characterize the surface geometry. ii) Can $\Delta u^{+}$be generalized to other flows.

Although many parameters influence the Hama roughness function $\Delta u^{+}[21$, 28-31], the most relevant parameter is the characteristic height of the roughness $k^{+}$. In a regime in which the pressure forces dominate the drag force, any surface can be collapsed onto the Nikuradse data by rescaling the roughness height to the so-called 'equivalent sandgrain roughness height' $k_{s}^{+}$. [10] found that three regimes of the characteristic roughness height $k_{s}^{+}$can be identified with respect to the effect of roughness [32]. For $k_{s}^{+} \lesssim 5$, the rough wall appears to be hydrodynamically smooth and the roughness function $\Delta u^{+}$goes to zero. For $k_{s}^{+} \gtrsim 70$, the wall drag scales quadratically with the fluid velocity and the friction factor $C_{f}$ is independent of the Reynolds number, indicating that hydrodynamic pressure drag (also called form drag) on the roughness dominates the total drag. The transitionally rough regime is in between these two regimes. Where in the fully rough regime, a surface is fully determined by $k_{s}^{+}$to give a collapse onto the fully rough asymptote [23], in the transitionally rough regime, different surfaces give rise to different roughness functions, see 
e.g. figure 3 in [6]. This can be attributed to the delicate interplay between pressure drag, viscous drag, and the weakening of the so-called turbulence generation cycle [6].

An intriguing feature of the data from [10] is at $k_{s}^{+} \approx 5$, where roughness effects suddenly result in an inflectional increase of $\Delta u^{+}$, as compared to the gradual increase of the roughness function found by [33] who extracted an empirical relationship from many industrial surfaces [28]. Later, this inflectional behavior was also observed for tightly packed spheres [19], honed surfaces [34], and grit-blasted surfaces [35]. [36] had too few points to find the inflectional behavior; however, their two simulations of monodisperse spheres in regular arrangement collapsed on the Nikuradse curve. In the Moody [37] representation, this inflectional behavior manifests itself as a dip in the friction factor $C_{f}$, leading to a significantly lower drag coefficient $(\approx 20 \%[38])$ in comparison to the monotonic behavior of [33], on which the original Moody diagram is based. Although it is proposed that the inflectional behavior has to do with the monodispersity of the roughness leading to a critical Reynolds number at which the elements become active [6], recent simulations by [35] for a polydisperse surface (containing irregularities with a range of sizes) also show this inflectional behavior. In a broader sense, the DNS by [35] are interesting since they show, for the first time, a surface that very closely resembles the [10] roughness function in all regimes, the authors found $k_{s}^{+}=0.87 k_{t}^{+}$, where $k_{t}$ is the mean peak-to-valley height.

Regarding TC flow, only a few studies have looked at the effect of roughness $[39,40]$. Recently, the effect of regular roughness on TC turbulence has also been investigated by means of DNS [41-43]. [41] looked at the effect of axisymmetric grooves on the torque scaling, boundary layer thickness, and plume ejections. They find that enhanced plume ejection from the roughness tips can lead to an ultimate torque scaling exponent of $N u \propto T a^{0.5}$, although for higher $T a$ the exponent saturates back to the ultimate scaling effective exponent of 0.38. [42] then simulate transverse bar roughness elements on the inner cylinder to disentangle the separate effects of viscosity and pressure, and find that the ultimate torque scaling exponent of $N u \propto T a^{0.5}$ is only possible when the pressure forces dominate at the rough boundary [43].

In contrast to the above mentioned previous work, in which the roughness consisted of well-defined transverse bars with constant distance and heights $[42,43]$, in this research we set out to investigate the effects of irregular, monodisperse roughness, resembling the sand grain roughness reported by [10]. We model the roughness as randomly rotated and semi-randomly translated 
ellipsoids of constant volume and aspect ratio, based on the roughness model (subgrid-scale) of [18]. Previously, a fully resolved version of the model by [18] was used for large-eddy simulations in channel flow [44]. Taylor numbers in our DNS range from $T a=1.0 \times 10^{7}\left(R e_{\tau}=82\right)$ to $T a=1.0 \times 10^{9}\left(R e_{\tau}=635\right)$; therefore, we capture both classical (laminar-like boundary layers) and ultimate (turbulent boundary layers) regimes $[13,43,45]$. Moreover, whereas previous research on roughness in TC flow focussed on the torque scaling, we now look at the effects of the roughness height on the Hama roughness function $\Delta u^{+}$in the transitionally rough and fully rough regimes, ranging from $k_{s}^{+}=5$ to $k_{s}^{+}=92$.

This chapter is structured as follows. In section $1.2 \&$ section 1.3 , we elaborate on the TC setup, the roughness model and the numerical procedure. In section 1.4.1, we study the velocity profiles and present the effects of the roughness height on the Hama roughness function. In section 1.4.2, we present the global response of the system. In section 1.4 .3 we study the flow structures. In section 1.4.4 the fluctuations close to the roughness are studied and in section 1.4.5 we present radial profiles of various other quantities. Finally, in section 1.5 we draw our conclusions and propose future research directions.

\subsection{Taylor-Couette flow}

The TC setup, as shown in figure 1.1, comprises independently co- or counterrotating concentric cylinders around their vertical axis. The flow, driven by the shear on both of the cylinders, fills the gap between the cylinders. $r_{i}$ is the inner cylinder radius, $r_{o}$ is the outer cylinder radius, and the radius ratio is defined as $\eta=r_{i} / r_{o}$. For this research, we set $\eta \approx 0.714$, to match the experimental Twente Turbulent Taylor-Couette $\left(\mathrm{T}^{3} \mathrm{C}\right)$ setup [27], and previous simulations [42]. $\Gamma=L / d$ is the aspect ratio, where $L$ is the height of the cylinders, and $d=r_{o}-r_{i}=0.4 r_{i}$ is the gap width. Here, $\Gamma \approx 2$ such that one pair of Taylor vortices fits in the gap, and the mean flow statistics become independent of the aspect ratio [46]. In the azimuthal direction we employ a rotational symmetry of order 6 to save on computational expense such that the streamwise aspect ratio of our simulations becomes $L_{\theta} / d=\left(r_{i} 2 \pi / 6\right) / d=2.62$. [47] and [46] found that this reduction of the streamwise extent does not affect the mean flow statistics. This gives $L_{\theta} /(d / 2)=5.24$ and $L /(d / 2) \approx 4.0$.

To maintain convenient boundary conditions, we solve the Navier-Stokes (NS) equations in a reference frame rotating with the inner cylinder $\left(\omega_{i} \mathbf{e}_{z}\right)$ The NS equations, with $(u, v, w)$ the (streamwise/azimuthal, spanwise/axial, wall- 
(a)

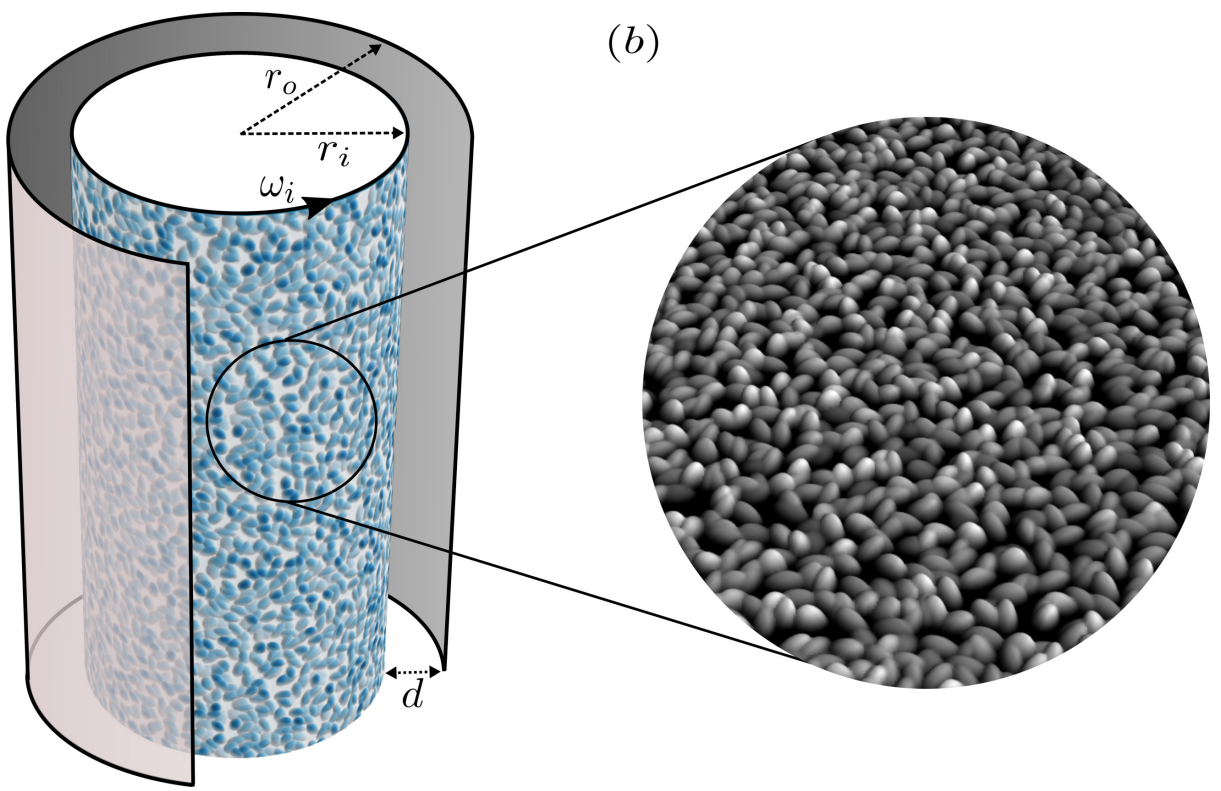

Figure 1.1: Illustration of the Taylor-Couette (TC) setup. (a) TC setup with inner cylinder sand grain roughness. $\omega_{i}$ is the inner cylinder angular velocity, $r_{i}$ is the inner cylinder radius, $r_{o}$ is the outer cylinder radius, and $d=r_{o}-r_{i}$ the gap width. (b) A zoom of the sand grain roughness that is modeled. The outer cylinder is stationary and smooth. 
normal/radial) velocity components respectively, in that reference frame become

$$
\begin{gathered}
\partial_{\hat{t}} \hat{w}+\hat{\mathbf{u}} \cdot \hat{\nabla} \hat{w}-\frac{\hat{u}^{2}}{\hat{r}}=-\partial_{\hat{r}} \hat{P}+\frac{f(\eta)}{T a^{1 / 2}}\left(\hat{\nabla}^{2} \hat{w}-\frac{\hat{w}}{\hat{r}^{2}}-\frac{2}{\hat{r}^{2}} \partial_{\theta} \hat{u}\right)-R o^{-1} \hat{u} \\
\partial_{\hat{t}} \hat{u}+\hat{\mathbf{u}} \cdot \hat{\nabla} \hat{u}-\frac{\hat{u} \hat{w}}{\hat{r}}=-\frac{1}{\hat{r}} \partial_{\hat{\theta}} \hat{P}+\frac{f(\eta)}{T a^{1 / 2}}\left(\hat{\nabla}^{2} \hat{u}-\frac{\hat{u}}{\hat{r}^{2}}+\frac{2}{\hat{r}^{2}} \partial_{\theta} \hat{w}\right)+R o^{-1} \hat{w} \\
\partial_{\hat{t}} \hat{v}+\hat{\mathbf{u}} \cdot \hat{\nabla} \hat{v}=-\partial_{\hat{z}} \hat{P}+\frac{f(\eta)}{T a^{1 / 2}}\left(\hat{\nabla}^{2} \hat{v}\right) \\
\hat{\nabla} \cdot \hat{\mathbf{u}}=0
\end{gathered}
$$

with no-slip boundary conditions $\left.u\right|_{r=r_{i}}=0,\left.u\right|_{r=r_{o}}=r_{o}\left(\omega_{o}-\omega_{i}\right)$. Equation (1.6) expresses the incompressible restriction. Hatted symbols indicate the respective dimensionless variables, with $\mathbf{u}=r_{i}\left|\omega_{i}-\omega_{o}\right| \hat{\mathbf{u}}, r=d \hat{r}$ and $t=\frac{d}{r_{i}\left|\omega_{i}-\omega_{o}\right|} \hat{t} \cdot \frac{f(\eta)}{T a^{1 / 2}}=R e^{-1}$ where $f(\eta)$ is the so-called 'geometric Prandtl' number (highlighting the analogy with the Prandtl number in the dimensionless Navier-Stokes equations of Raleigh-Bénard convection) [48]:

$$
f(\eta)=\frac{(1+\eta)^{3}}{8 \eta^{2}}
$$

here $f(0.714) \approx 1.23 . T a$ is the Taylor number, which is a measure of the driving strength of the system,

$$
T a=\frac{(1+\eta)^{4}}{64 \eta^{2}} \frac{\left(r_{o}-r_{i}\right)^{2}\left(r_{i}+r_{o}\right)^{2}\left(\omega_{i}-\omega_{o}\right)^{2}}{\nu^{2}} .
$$

Note that the pressure in the equations above represents the 'reduced pressure' that incorporates the centrifugal term; $\hat{P}=p^{\prime}-\frac{\omega_{i}^{2} d^{2} \hat{r}^{2}}{2 r_{i}^{2}\left|\omega_{i}-\omega_{o}\right|^{2}} \mathbf{e}_{r}$ with $p=\rho r_{i}^{2} \mid \omega_{i}-$ $\left.\omega_{o}\right|^{2} p^{\prime}$ and $p$ is the physical pressure. It is directly clear that the centrifugal force in TC flow is analogous to the gravitational force in RB flow [48]. The final term on the right-hand side of eq. (1.3) and (1.4) gives the Coriolis force, with $R o^{-1}$ being the inverse Rossby number

$$
R o^{-1}=\frac{2 \omega_{i} d}{r_{i}\left|\omega_{i}-\omega_{o}\right|} .
$$

Analogous to RB flow, the global response of TC flow can be expressed in terms of a Nusselt number. In the former, the Nusselt number expresses the 
dimensionless conserved heat flux, whereas in the latter the Nusselt number expresses the dimensionless conserved angular velocity flux $J^{\omega}$, calculated by:

$$
J^{\omega}=r^{3}\left(\langle w \omega\rangle_{\theta, z, t}-\nu \partial_{r}\langle\omega\rangle_{\theta, z, t}\right)
$$

with the laminar flux given by $J_{l a m}^{\omega}=2 \nu r_{i}^{2} r_{o}^{2} \frac{\omega_{i}-\omega_{o}}{r_{o}^{2}-r_{i}^{2}}$ where $\nu$ is the kinematic viscosity and $\langle\cdot\rangle_{\theta, z, t}$ indicates averaging over the spatial directions $\theta, z$ and time $t$. For incompressible flows, it can be derived from the NS equations that $J^{\omega}$ is conserved in the radial direction, $\partial_{r} J^{\omega}=0$ [48]. In both cases, the values are made dimensionless by their respective laminar, conducting, values. For TC flow the Nusselt number becomes:

$$
N u_{\omega}=\frac{J^{\omega}}{J_{l a m}^{\omega}}
$$

The angular velocity flux $J^{\omega}$ can be written in terms of the torque $\mathcal{T}$ on any of the cylinders: $J^{\omega}=\mathcal{T}(2 \pi L \rho)^{-1}$ with $\rho$ being the fluid density. Consequently, the shear stress on the inner cylinder $\tau_{w, i}$ is related to the angular velocity flux by $\tau_{w, i}=\frac{\rho J^{\omega}}{r_{i}^{2}}$.

Since part of our endeavour is to compare the effects of sand grain roughness on TC turbulence with the effects of sand grain roughness in other canonical systems (e.g. pipe flow), where the use of $N u_{\omega}$ is not conventional, we choose to also present the global response in terms of the friction factor $C_{f}$. Here we follow [49], and define $C_{f} \equiv G / R e_{i}^{2}$, where $G$ is the dimensionless torque $G=\mathcal{T} /\left(\rho \nu^{2} L\right)$ and $R e_{i}$ is the inner cylinder Reynolds number $R e_{i}=\frac{r_{i} \omega_{i} d}{\nu}$. The translation between $N u_{\omega}$ and $C_{f}$ is straightforward;

$$
C_{f} \equiv \frac{2 \pi \tau_{w, i}}{\rho d^{2}\left|\omega_{i}-\omega_{o}\right|^{2}}=2 \pi N u_{\omega} J_{\text {lam }}^{\omega}\left(\nu R e_{i}\right)^{-2}
$$

Note that one can also define $C_{f} \equiv 2 \tau_{w, i} /\left(\rho\left(r_{i} \omega_{i}\right)^{2}\right)=\frac{(1-\eta)^{2}}{\pi \eta^{2}} \frac{G}{R e_{i}^{2}}$, which is different from eq. (1.12) by a factor which depends on the radius ratio $\eta$ [49]. Here we use the definition of $C_{f}$ of eq. 1.12.

\subsection{Numerical procedure}

\subsubsection{Roughness model}

Figure 1.2 exhibits the setup of the 'virtual' sand grain roughness model that is used in this research. The inner cylinder is divided up into square tiles of 
(a)

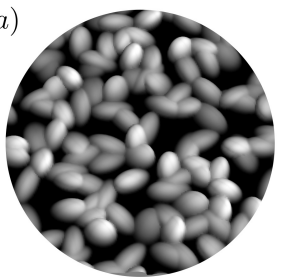

$(d)$ (b)

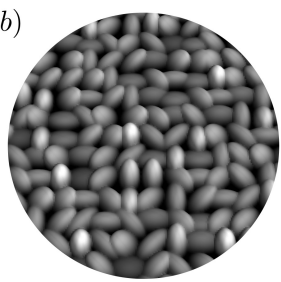

(c)

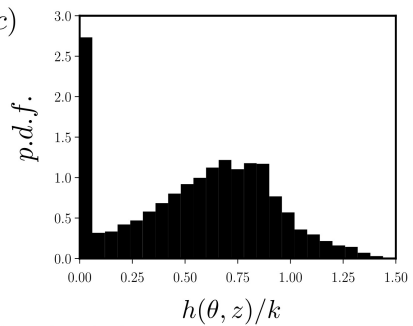

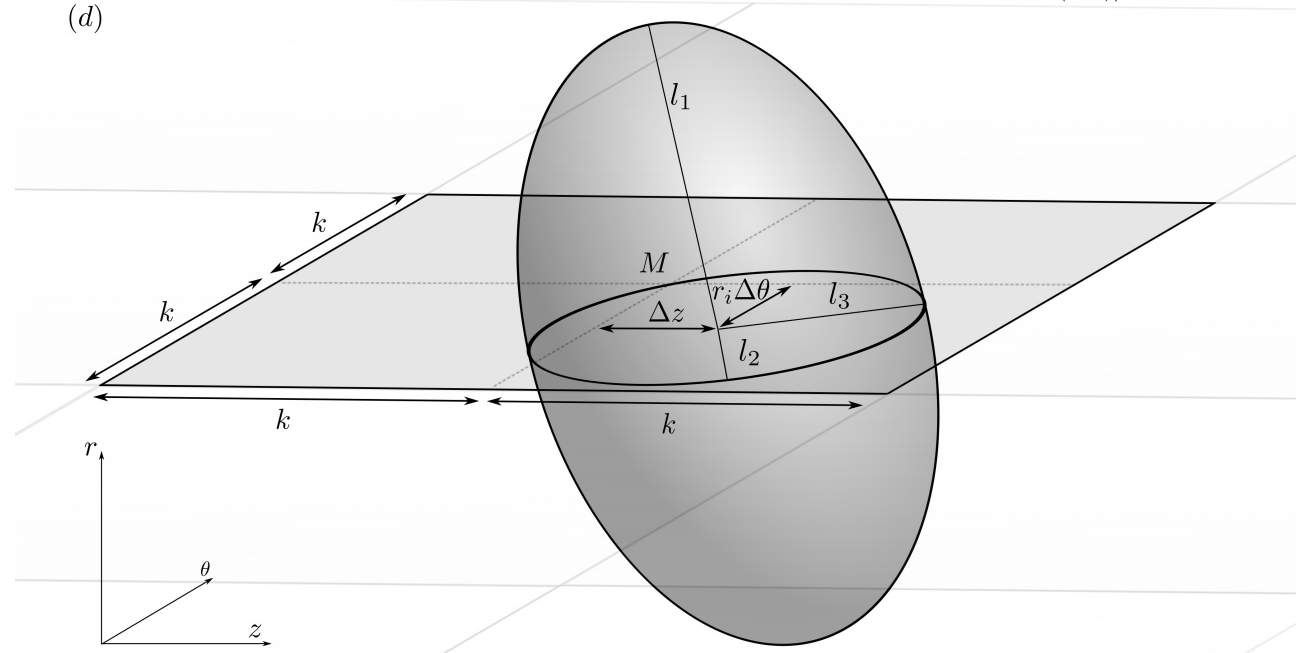

Figure 1.2: (a) Visual comparison between a surface with translational degrees of freedom as employed presently and $(b)$ the original model by [18] without translational degrees of freedom. (c) Probability density function (p.d.f.) of the surface height $h(\theta, z) / k$ distribution of a rough surface with 952 roughness elements (B2). For the statistics of all rough surfaces used in this study, we refer the reader to the Appendix. (d) Schematic of an ellipsoidal building block of the rough surface. Every rectangular tile of size $2 k \times 2 k$ contains exactly one ellipsoid. $M$ indicates the center of the tile. The radii $l_{1}=2.0 k, l_{2}=$ $1.4 k, l_{3}=1.0 k$ are kept constant for each ellipsoid to maintain a monodisperse rough surface. Randomness is ensured by giving the ellipsoid 5 degrees of freedom; 2 translational shifts of the center of the ellipsoid from the center of the tile $M,\left(\Delta z\right.$ and $\left.r_{i} \Delta \theta\right)$ and 3 rotational degrees of freedom $\left(\alpha_{1}, \alpha_{2}, \alpha_{3}\right)$ from $(r, \theta, z)$ to $\left(l_{1}, l_{2}, l_{3}\right)$. We also employ a constant translation of the center of the ellipsoid in the radial direction, with $\Delta r=-0.5 k$ from $r=r_{i}$. 
size $2 k \times 2 k$, each tile containing exactly 1 ellipsoid, with $k$ the length of the minor radius of the ellipsoid. The height $L$ is slightly varied $\left(0.85 r_{i} \pm 0.03 r_{i}\right)$ to ensure that an integer amount of tiles fits into the domain. Unlike in the original model by [18], we also introduce a random translation of the center of the ellipsoid by applying $r_{i} \Delta \theta$ and $\Delta z$, where $r_{i} \Delta \theta$, and $\Delta z$ are random uniform translations from the center of the $2 k \times 2 k$ tile. This random translation allows for the surface to be more irregular and as such to relate more closely to a realistic sand grain surface. As also introduced by [18], we employ a constant translation of the center of the ellipsoid in the radial direction, with $\Delta r=-0.5 k$ from $r=r_{i}$. It is shown in figure 1.1(a) that part of the cylinder $(\approx 15 \%)$ is not covered by rough elements. The projected area of the ellipsoids equals the area of the inner cylinder that is rough. This means that the surface is not 'overhanging', resulting from the offset of the center of the ellipsoid in the radial direction $\Delta r=-0.5 k$. This makes the computations significantly less involved. By saying that part of the surface is not covered by rough elements, we mean that the neighbouring ellipsoids don't close the entire surface. This could be achieved by stacking multiple layers of ellipsoids. Statistics of the rough surfaces are found in the Appendix, Table 2.

\subsubsection{Numerical method}

The NS equations are spatially discretized by using a central second-order finite-difference scheme and solved in cylindrical coordinates by means of a semi-implicit procedure $[50,51]$. The staggered grid is homogeneous in both the spanwise and streamwise directions (the axial and azimuthal directions respectively). We apply no-slip boundary conditions at the cylinder walls and axially periodic boundary conditions at the top and bottom.

The wall-normal grid consists of a double cosine (Chebychev-type) grid stretching. Below the maximum roughness height, we employ a cosine stretching such that the maximum grid spacing is always smaller than 0.5 times the viscous length scale. In the bulk of the fluid, we employ a second stretching, such that the maximum grid spacing in the bulk is approximately 1.5 times the viscous length scale. The minimum grid spacing is located at the position of the maximum roughness height, where we expect the highest shear stress, see table 1.1 for the exact values.

Time advancement is performed by using a fractional-step third-order RungeKutta scheme in combination with a Crank-Nicolson scheme for the implicit terms. The Courant-Friedrichs-Lewy (CFL) $\left(\frac{U \Delta t}{\Delta x}<0.8\right.$, with $\Delta x$ being the grid size, and $U$ the velocity, both in the respective coordinate direction) 
condition is tested in all directions and accordingly the time-step constraint for the non-linear terms is enforced to ensure stability.

Sand grain roughness is implemented in the code by an Immersed Boundary Method (IBM) [52]. In the IBM, the boundary conditions are enforced by adding a body force $\mathbf{f}$ to the momentum equations $(1.3-1.5)$. A regular, non-body fitting, mesh can thus be used, even though the rough boundary has a very complex geometry. We perform linear interpolation in the spatial direction for which the component of the normal surface vector is largest. The IBM has been validated previously [41-43, 52-54].

Simulations run until they become statistically stationary, such that Nusselt $N u_{\omega}$ number remains constant to within $\approx 1 \%$, which requires $\hat{t} \approx 200$. Thereafter, we gather statistics until the results converge, which requires $\hat{t} \approx$ 50. The resolution constraints of the domain are typically derived from the literature and are based on the grid spacing in '+' (viscous) units. Grid independence checks of the time-averaged statistics are performed by ensuring that $N u_{\omega}$ remains constant with increasing grid resolution in all directions and presented along with the results in Table 1.1. Throughout the chapter we employ superficial averaging - both over solid and fluid regions - unless stated otherwise.

\subsection{Results}




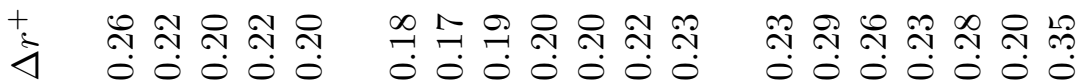

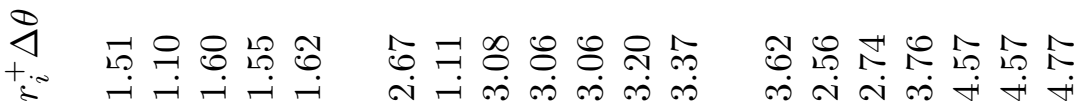

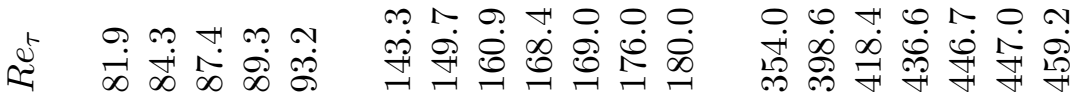

+

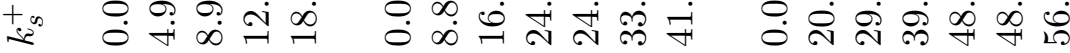

\}

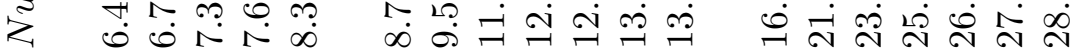

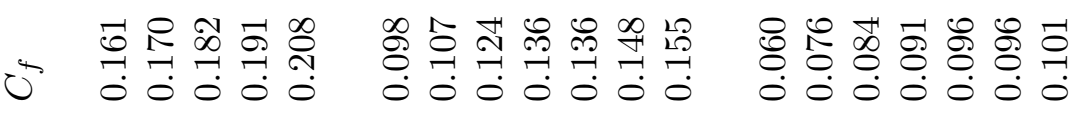

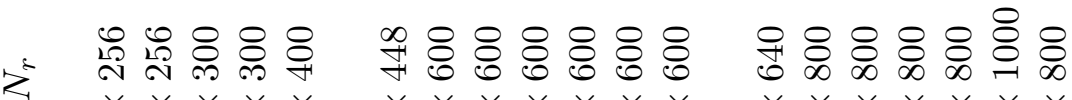

$\times \times \times \times \times \times \times \times \times \times \times \times \times \times \times \times \times \times \times \times$

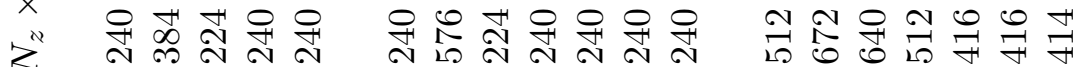

$\times \times \times \times \times \times \times \times \times \times \times \times \times \times \times \times \times \times$

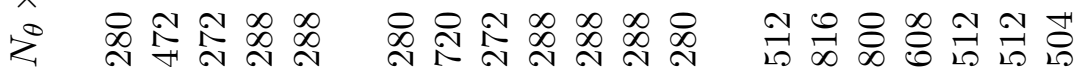

z $\quad$ |

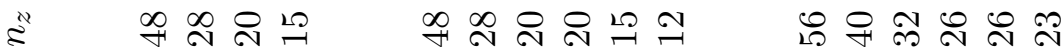

$\times \quad \times \times \times \times \times \times \times \times \times \times \times \times \times \times$

¿ 1 है

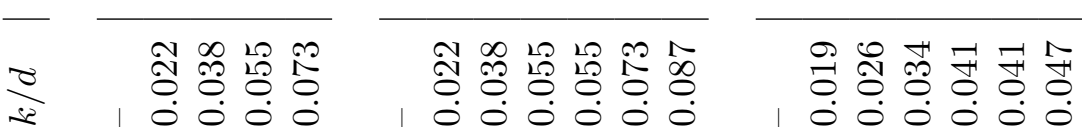

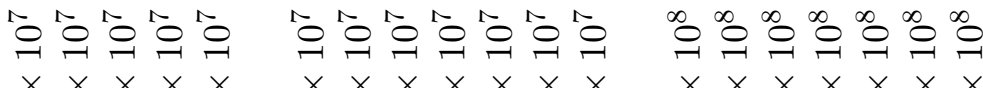

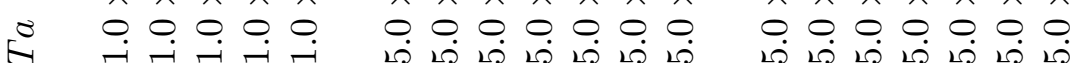

ঠ் 


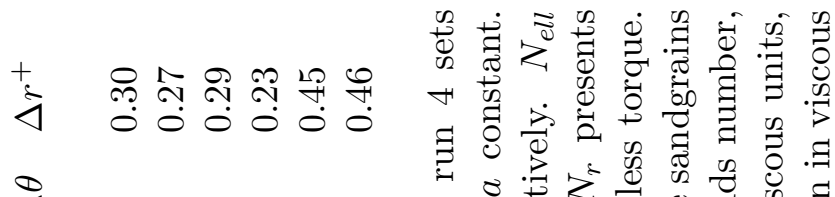

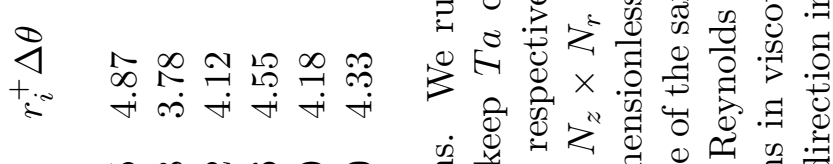

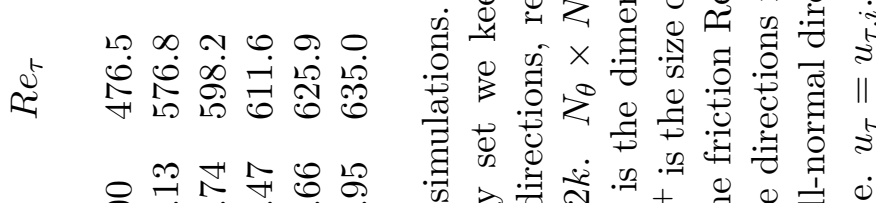

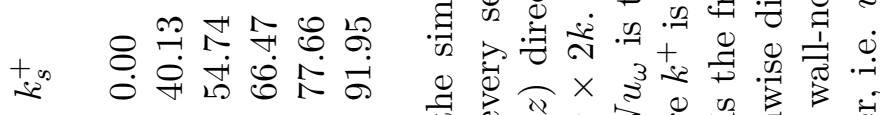

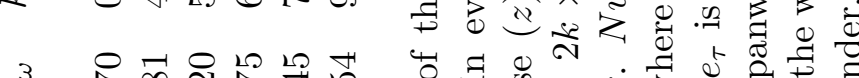

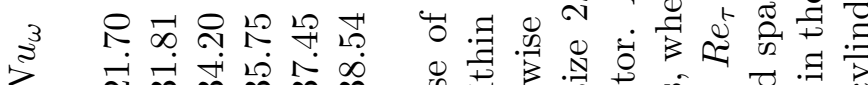

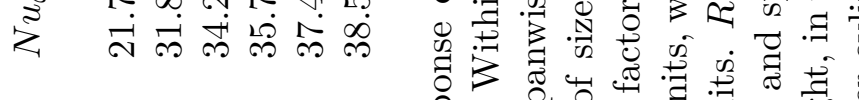

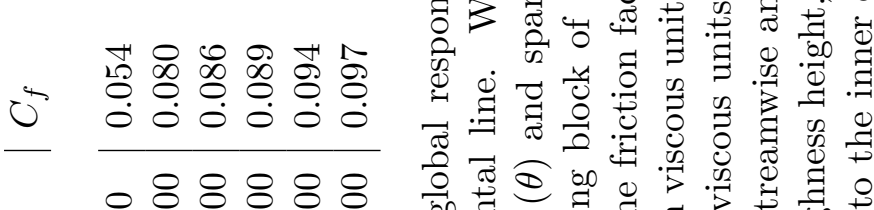

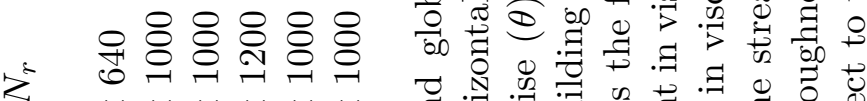

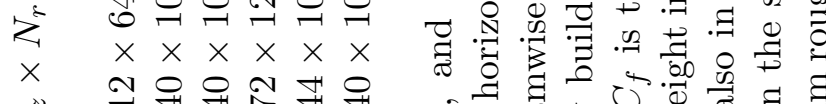

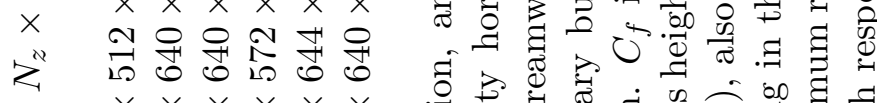

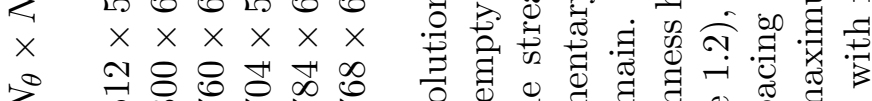

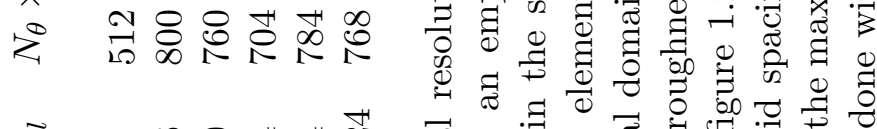

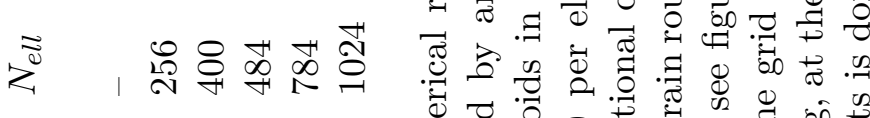

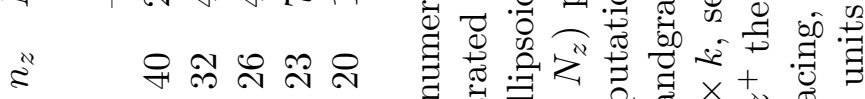

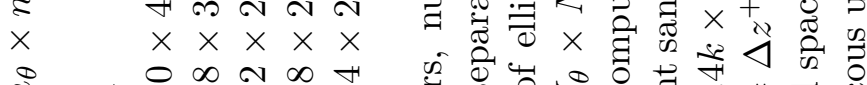

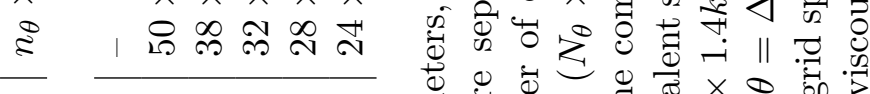

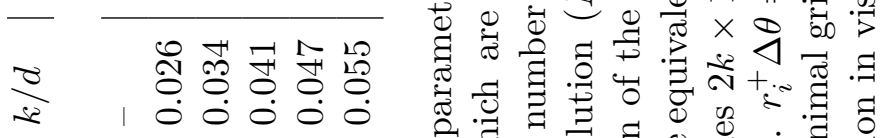

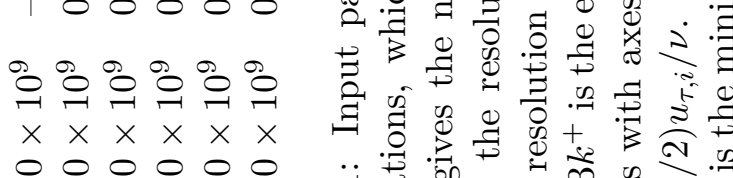
\& OO

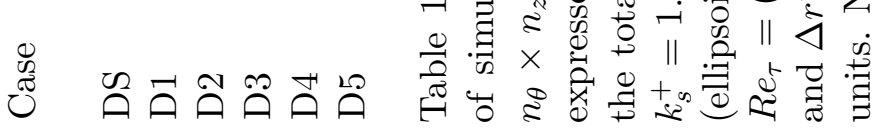




\subsubsection{Roughness function}

Figure 1.2 (left column) presents the streamwise (i.e. azimuthal) velocity profiles $u^{+}=\left\langle u(r)-u\left(r_{i}\right)\right\rangle_{\theta, z, t} / u_{\tau}$ (solid) and angular velocity profiles $\omega^{+}=$ $\left\langle u\left(r_{i}\right)-r_{i} u(r) / r\right\rangle_{\theta, z, t} / u_{\tau}$ (dashed) versus the wall normal distance $y^{+}=r^{+}-$ $r_{i}^{+}-h_{m}^{+}$, where $\langle.\rangle_{\theta, z, t}$ indicates averaging over the streamwise and spanwise directions and in time, and $h_{m}^{+}$is the mean roughness height. Every row corresponds to simulations at constant rotation rate of the inner cylinder (Taylor number), and increasing roughness height.

In line with the previous observations of [27] and [45], we also find that the logarithmic profiles of the streamwise velocity $u^{+}$in smooth-wall TC do not fit the $\kappa=0.4$ slope, as found in other wall bounded flows (e.g. pipe, boundary layer, channel) - for similar values of the friction Reynolds number $R e_{\tau}$. However, this asymptotic value is experimentally observed at very high shear rates of $T a=O\left(10^{12}\right)$ and $R e_{\tau}=O\left(10^{4}\right)$ [27], much higher than can be obtained by the present DNS. The logarithmic profiles of angular velocity $\omega^{+}$have a slope that is closer to the $\kappa=0.4$ asymptote [55], especially for the higher Ta figure $1.2(g, h)$. Here we investigate the effects of roughness on both $u^{+}$and $\omega^{+}$.

For rough wall simulations, the logarithmic region shifts downwards - a hallmark effect of a drag increasing surface. Figure 1.2 (right column) present the shifts, where $\Delta u^{+}=u_{s}^{+}-u_{r}^{+}$and $\Delta \omega^{+}=\omega_{s}^{+}-\omega_{r}^{+}$. All shifts of the angular and azimuthal profiles are calculated at the center of the gap, that is at $y^{+}+h_{m}^{+}=R e_{\tau}$. As one can see in figures 3 (b,d,f,h) of the chapter, the values of $\Delta u^{+}$and $\Delta \omega^{+}$do not depend on $y^{+}$if one is sufficiently far away from the wall. For lower $T a$, there is a small but observable difference between $\Delta u^{+}$and $\Delta \omega^{+}$, see figure $1.2(b)$, whereas for the higher $T a$, this difference diminishes, see figure $1.2(f, h)$.

Figure 1.3 $(a, b)$ presents the shift of the streamwise and angular velocity profiles, respectively, versus the equivalent roughness height $k_{s}^{+}$, for all $T a$. Care is taken to ensure overlap for varying $T a$ and similar $k^{+}$, to study the $T a$ dependence of $\Delta u^{+}$and $\Delta \omega^{+}$. However, despite the varying Ta numbers, all data collapse onto a single curve, with some scatter. Note that scatter is expected due to the randomness of the surfaces, which are reproduced for every simulation. To obtain an estimate of the expected scatter, we run two simulations with statistically similar surfaces. These are indicated by B3 and BY in table 1.1 and the velocity profiles are found in figures $1.2(c, d)$. We find a difference between the two cases of $\lesssim 0.2 \Delta u^{+}, 0.2 \Delta \omega^{+}$. The measure of this scatter is indicated in figure 4 as a vertical bar (= spread). We conclude that 

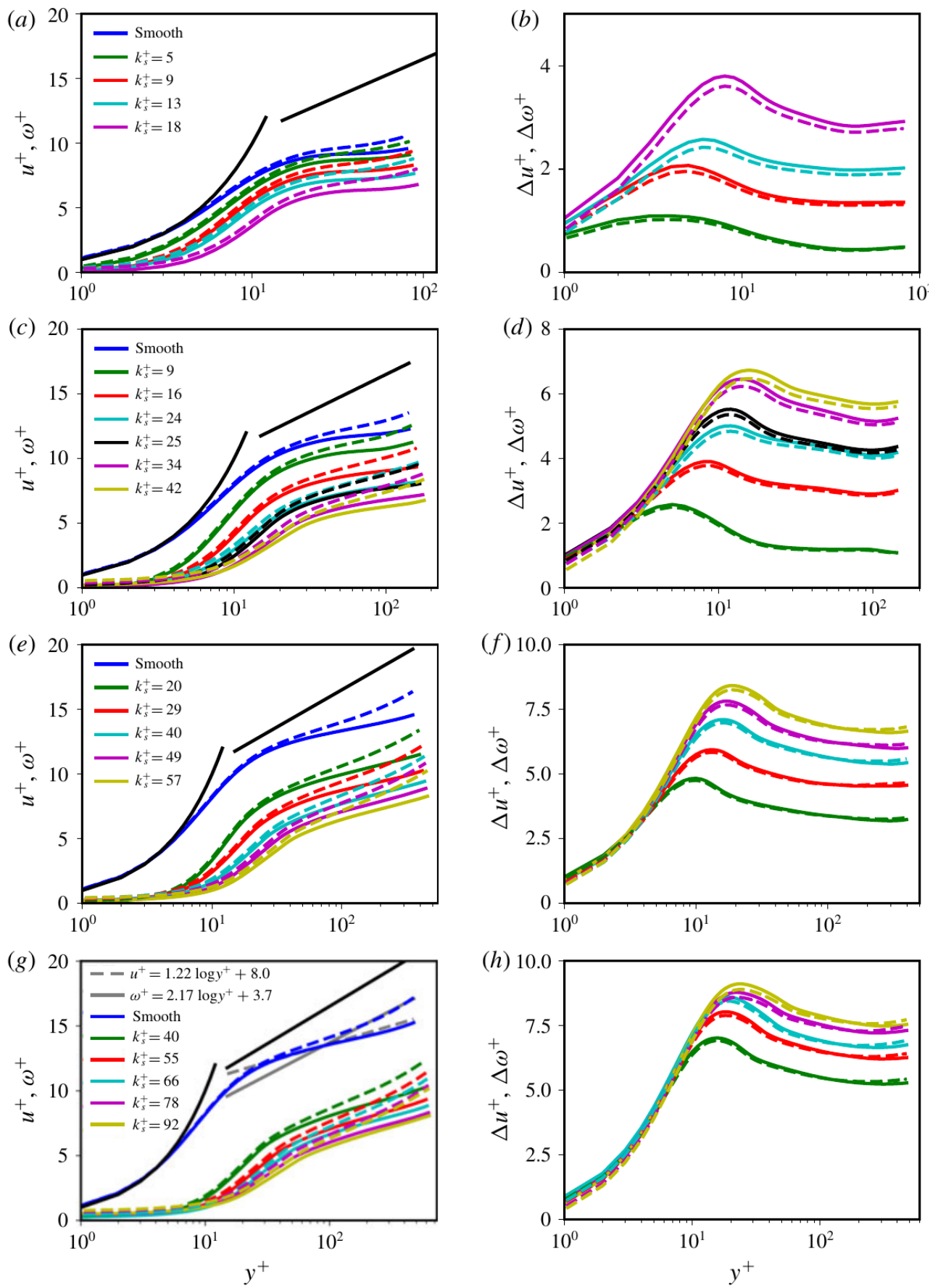
Figure 1.2: $(a, c, e, g)$ Profiles of the streamwise - azimuthal - velocity $u^{+}($solid $)$ and the angular velocity $\omega^{+}$(dashed) versus the wall normal distance $y^{+}$. Black solid lines indicate the viscous sublayer profile $u^{+}=y^{+}$and the logarithmic law $u^{+}=\kappa^{-1} \log y^{+}+B$, with $\kappa=0.4$ and $B=5.0 . \quad(b, d, f, h)$ Profiles of the streamwise velocity shift $\Delta u^{+}$(solid) and the angular velocity shift $\Delta \omega^{+}$(dashed). Every row corresponds to a constant Taylor number, $(a, b)$ $T a=1.0 \times 10^{7},(c, d) T a=5.0 \times 10^{7},(e, f) T a=5.0 \times 10^{8}$ and $(g, h)$ $T a=1.0 \times 10^{9}$, see Table 1.1. The grey lines in $(g)$ are logarithmic fits to the smooth profiles for $y^{+}=[150,500]$.

the variability in $\Delta u^{+}$and $\Delta \omega^{+}$at constant $k^{+}$and varying $T a$ falls within the size of that vertical bar, and such conclude that $\Delta u^{+}$and $\Delta \omega^{+}$and thus the equivalent roughness height $k_{s}^{+}$shows little dependence on $\mathrm{Ta}$.

A comparison with the findings of [10] can be carried out by scaling the fully rough regime to obtain $k_{s}^{+}=C k^{+}$, where $C$ is a constant that depends on the surface topology. In figure 1.4(a) we plot the velocity profiles versus $(r-$ $\left.h_{m}\right) / k_{s}$ for the highest roughness (D3, D4 and D5 respectively, see Table 1.1). Excellent collapse of the D4 and D5 profiles indicates that those simulations are indeed fully rough. In this fully rough regime viscosity can be neglected $\left(y \gg k \gg \delta_{\nu}\right)$, whereas the velocity profile is also independent of the system outer length scales $(y \ll d)$ i.e. the overlap argument [11]. The gradient of the velocity profile becomes; $\frac{d\langle U\rangle}{d y}=\frac{u_{\tau}}{y} \Phi(y / k)$, where $\Phi(y / k)$ is a universal function that will go to $1 / \kappa$. Integration then gives;

$$
u_{r}^{+}=\frac{1}{\kappa} \log (y / k)+B=\frac{1}{\kappa} \log \left(y / k_{s}\right)+\tilde{B}
$$

where $B$ is a constant and $y=r-h_{m}$. $\tilde{B}$ is the Nikuradse constant. The roughness function in the fully rough regime, (i.e. the fully rough asymptote), is obtained by subtracting (1.13) from the smooth wall profile $u_{s}^{+}=$ $(1 / \kappa) \log \left(y^{+}\right)+A$ and rescaling it to overlap with the Nikuradse data:

$$
\Delta u^{+}=\frac{1}{\kappa} \log \left(k_{s}^{+}\right)+A-\tilde{B} .
$$

In figure $1.3(a)$, the blue solid line is the fully rough asymptote, with $\kappa, A, \tilde{B}$ as found in pipe flow [11]. The green solid line is the fully rough asymptote as obtained from our simulations. $\kappa_{u}^{-1}=1.22$ and $A_{u}=8.0$ are taken from the fit of the smooth wall simulation at identical $T a$ as the fully rough cases 

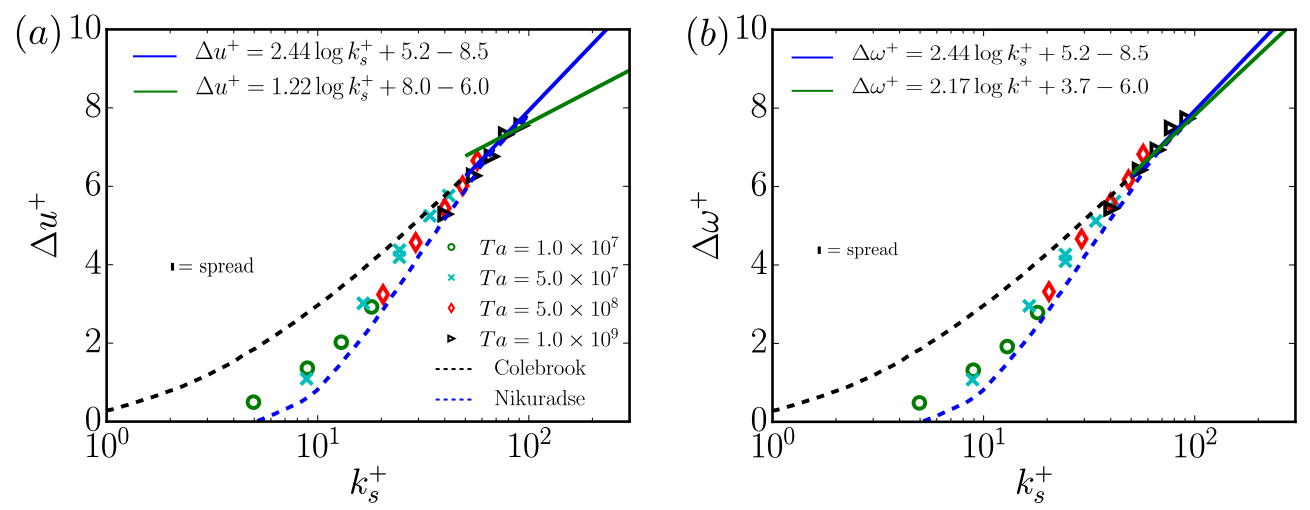

Figure 1.3: (a) Azimuthal velocity shift (Hama roughness function) $\Delta u^{+}$versus the equivalent sand grain roughness height $k_{s}^{+}$, where $k_{s}^{+}=1.33 k^{+}$. (b) Angular velocity shift $\omega^{+}$versus $k_{s}^{+}$. Close overlap with the Nikuradse curve is observed in the transitionally rough regime. The overlap is slightly better for the angular velocity shift, for which we also obtain $k_{s}^{+}=1.33 k^{+}$. The solid blue line represents the fully rough asymptote; $\Delta u^{+}=2.44 \log \left(k_{s}^{+}\right)+5.2-8.5$. The green lines represent the fully rough asymptotes obtained from the simulations, with $\kappa_{u}, \kappa_{\omega}, A_{u}$ and $A_{\omega}$ extracted from figure $1.2(\mathrm{~g})$. The spread between statistically similar surfaces, with similar mean and maximum heights, is indicated by the vertical bar. $k_{s}$ is determinded by a best fit between the two data points in the fully rough regime (i.e. Case D4 and D5, see table 1) and the Nikuradse fully rough asymptote. 

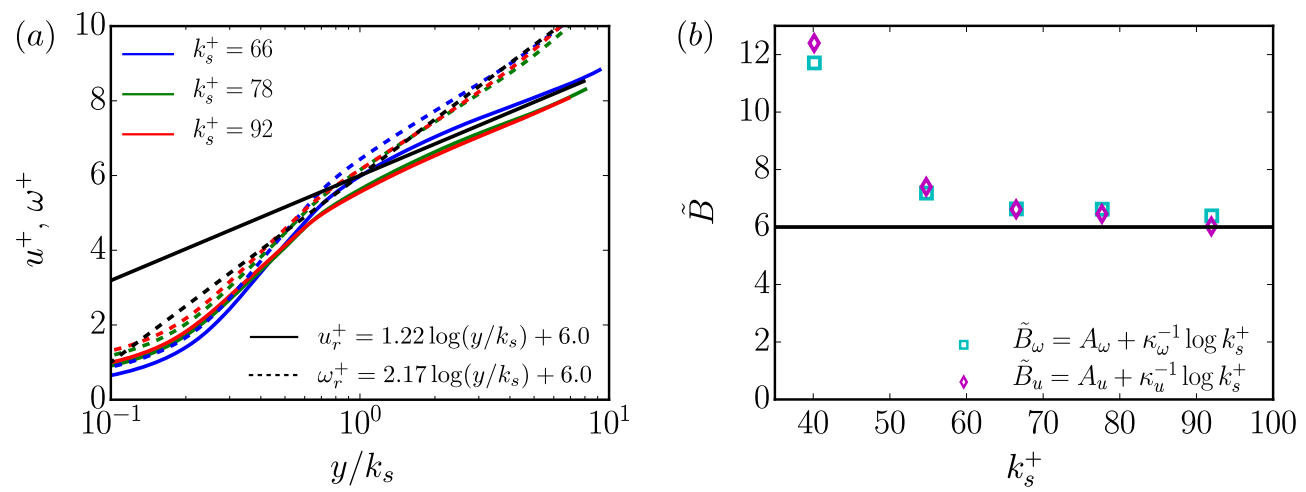

Figure 1.4: (a) Azimuthal velocity $u^{+}$(solid) and the angular velocity $\omega^{+}$ (dashed) versus the wall normal distance $y / k_{s}$, where $y=\left(r-h_{m}\right)$ and $h_{m}$ is the mean roughness height. The three simulations with the highest roughness are plotted (D3, D4 and $D 5$ respectively, see Table 1.1) to convey collapse of the profiles for the fully rough cases. (b) The Nikuradse constant $\tilde{B}$ versus the equivalent sand grain roughness height $k_{s}^{+}$for both the azimuthal velocity (squares) and the angular velocity (diamonds). Horizontal black line at $\tilde{B}=$ 6.0 gives the asymptotic value that is observed for fully rough behavior.

(figure $1.2 g$ ). The fits are in the domain $y^{+}=[150,500]$, as there the slope becomes approximately constant (figure $1.2 h$ ). $\tilde{B}$ is plotted in figure $1.4(b)$, where we find that $\tilde{B} \approx 6.0$ for the fully rough cases. The mismatch of the slopes in the fully rough regime makes a rescaling to find $k_{s}^{+}$a priori impossible - a statement that we wish to emphasize. However, to proceed with the comparison of the transitionally rough cases in TC and pipe flow, we choose to rescale the fully rough cases (D4 and D5) with the Nikuradse fully rough asymptote in figure 1.3 . We find that $k_{s}^{+}=1.33 k^{+}$and very close collapse of our data with the Nikuradse data.

In parallel, we analyse the behavior of $\Delta \omega^{+}$versus $k_{s}^{+}$, shown in figure 1.3(b). Again, the blue solid line represents the fully rough asymptote of Nikuradse. The green solid line is the fully rough asymptote obtained from fits $\left(y^{+}=\right.$ $[150,500])$ of the smooth wall angular velocity profile at identical $T a$ as the fully rough cases, see figure $1.2(\mathrm{~g})$. We find $\kappa_{\omega}^{-1}=2.17\left(A_{\omega}=3.7\right)$, close to the asymptotic value $\kappa^{-1}=2.44$. Although the differences are marginal, $\Delta \omega^{+}$ fits to the Nikuradse data slightly better than $\Delta u^{+}$(note that also here the rescaling is, $k_{s}=1.33 k$ ). However, the major difference is the closeness of the fully rough asymptotes. 
These results suggest that the near-wall effects of transitionally rough sand grains (and other rough surfaces) in TC flow are similar to the effects of transitionally rough sand grains (and other rough surfaces) in pipe flow (and other canonical systems). Further, we find that these transitionally rough effects are independent of slope of the velocity profile in the logarithmic region, whereas in the fully rough regime, they, a priori, depend on this slope. This is confirmed with similar values of $\Delta u^{+}$at similar $k_{s}^{+}$, for varying $T a$, see figure 1.3. Also the similarity between $\Delta u^{+}$and $\Delta \omega^{+}$in the transitionally rough regime confirms this, whereas the fully rough asymptotes are very dissimilar. We like to point out that simulations (or experiments) at high enough $T a$ $\left(=10^{12}[27]\right)$, where $\kappa=0.4$, then are expected to also give a collapse to the Nikuradse data in the fully rough regime.

\subsubsection{Global response}

Figure 1.5 $(a)$ presents the friction factor $C_{f}$ versus the dimensionless roughness height $k / d$ for varying $R e_{i}$. For lower $T a$ numbers, the friction factor $C_{f}$ decreases with increasing $T a$, indicating the relevance of viscous drag. For the two highest $T a$ numbers, representing the higher $k_{s}^{+}$cases, the friction factor almost collapses onto one line. This tells that $\tau_{w} \propto u^{2}$, and thus that pressure drag is dominant over viscous drag, in accordance with the overlap argument presented above. For constant $T a$, as expected, the friction factor increases for increasing roughness height. Figure 1.5(b) presents the global response in terms of $N u_{\omega}$. We observe an increase in $N u_{\omega}$ for increasing $T a$, corresponding to the increased transport of the angular velocity that is due to the increased turbulent mixing. Higher roughness leads to increased $J^{\omega}$ as compared to the smooth wall at the same $T a$, which also relates to a higher intensity of the turbulent mixing (the $r^{3}\left(\langle w \omega\rangle_{\theta, z, t}\right)$ term of equation (1.10)) and more plumes ejecting from the boundary layer radially outwards [42], on which we will elaborate in $§ 1.4 .3$.

By assuming a logarithmic profile, and integrating this profile over the entire gap (thereby neglecting the contributions of the viscous sublayer), we arrive at an implicit equation for the friction factor $C_{f}$, namely the celebrated Prandtl's friction law:

$$
\left(C_{f} / 2\right)^{-1 / 2}=C_{1} \log \left(\left(C_{f} / 2\right)^{1 / 2} R e_{i}\right)+C_{2}
$$

where $C_{1}\left(R e_{i}\right)$ and $C_{2}\left(R e_{i}\right)$ for TC at these $R e_{i}$. Figure 1.6 shows the friction factor $C_{f}$ versus the inner cylinder Reynolds number $R e_{i}$, for both smooth wall (solid) as the rough wall (symbols) simulations. An upward shift of the 

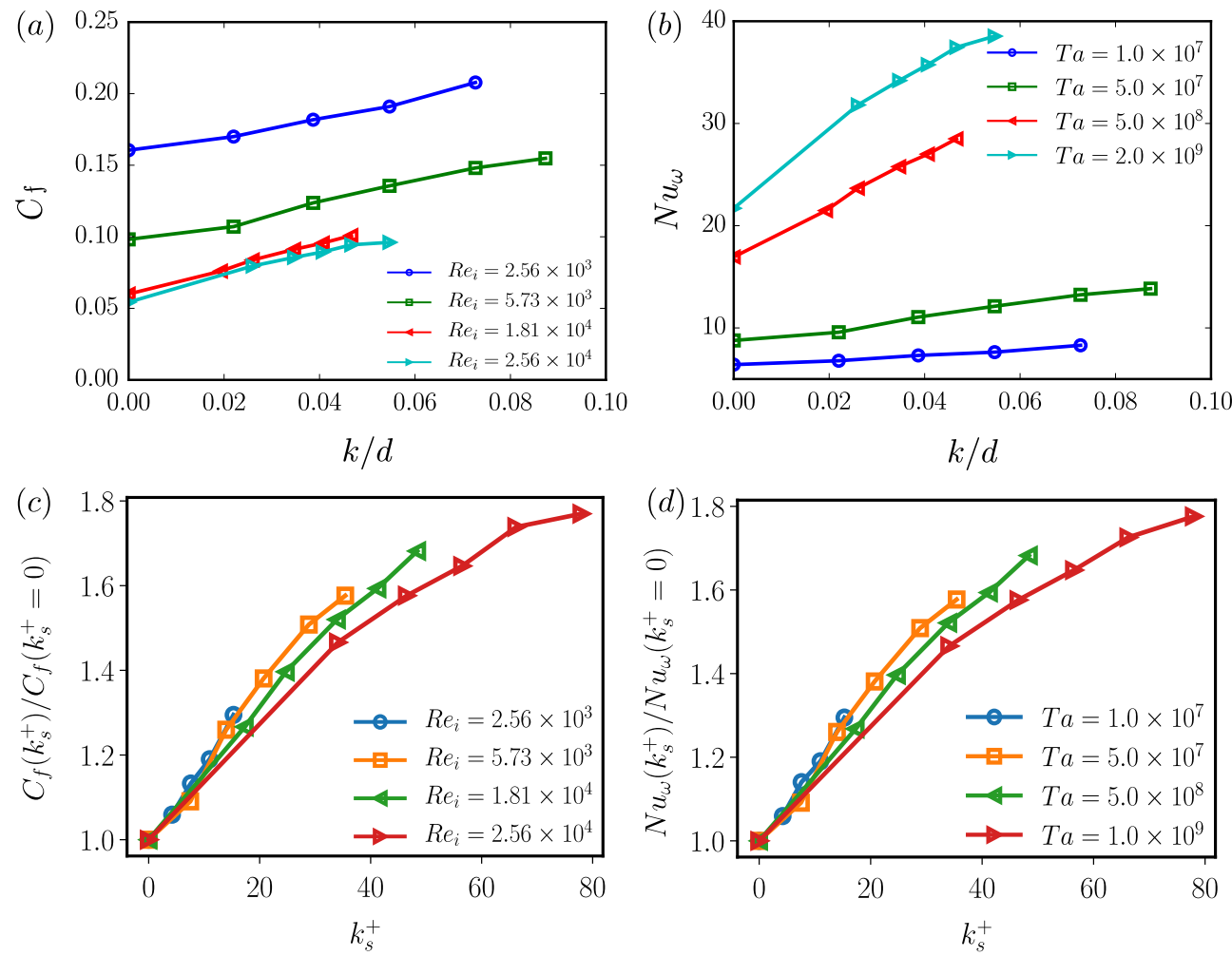

Figure 1.5: Profiles of $(a)$ the friction factor $C_{f}$, and $(b)$ the Nusselt number $N u_{\omega}$ versus the roughness height. $k / d$ is the roughness height $k$ relative to the gap width $d$. (c) Normalized friction coefficient $C_{f}\left(k_{s}^{+}\right) / C_{f}\left(k_{s}^{+}=0\right)$ versus the equivalent sand grain roughness height $k_{s}^{+}$. (d) Normalized Nusselt number $N u_{\omega}\left(k_{s}^{+}\right) / N u_{\omega}\left(k_{s}^{+}=0\right)$ versus the equivalent sand grain roughness height $k_{s}^{+}$. 

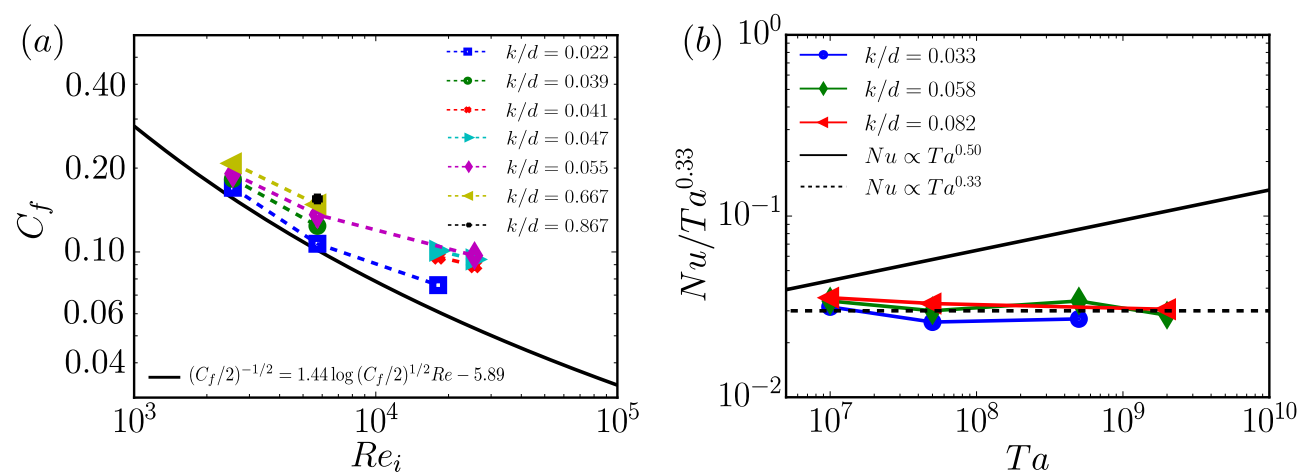

Figure 1.6: (a) Moody representation, showing the friction factor $C_{f}$ as a function of the inner cylinder Reynolds number $R e_{i}$ for varying roughness height $k / d$. The solid line is the fit of the Prandtl friction law to the smooth wall simulation data. (b) Compensated plot of the Nusselt number versus the Taylor number for constant $k / d$. In this regime, $N u \propto T a^{0.33}$. The solid black line indicates the assymptotic scaling of $N u \propto T a^{0.50}$.

friction factor for increasing roughness height is consistent with what is observed for sand grains in pipe flow [10] and recently also for tranverse ribs in Taylor-Couette flow [43]. Note that this upward shift, is directly related to the downward shift of the mean streamwise velocity profile (the roughness function). Since the friction factor and the Nusselt number are related, as expressed in equation (1.12), we expect the Nusselt number to increase, for increasing roughness height. This is confirmed in figure 1.6(b), where we plot the $\mathrm{Nu}$ number versus the Ta number. The number of simulations with constant $k / d$ is limited, and we vary the Ta number over 2 decades only. However, we observe that the asymptotic, ultimate scaling of $N u_{\omega} \propto T a^{0.5}$, as found for fully rough transverse ribs in [43], is not reached. This is expected, as only the inner cylinder is covered with roughness.

\subsubsection{Flow structures}

To obtain a qualitative understanding of the effect of inner cylinder roughness on the turbulent flow in the gap, we present two series of snapshots of the streamwise azimuthal velocity $u(r, \theta, z, t)$. Figure $1.7(a-c)$ exhibit the snapshots for $T a=5.0 \times 10^{7}$. It is known, and observed here, that for this Taylor number the coherence length of the dominant flow structures becomes smaller than the gap width $d$, and turbulence develops in the bulk [13]. On the 

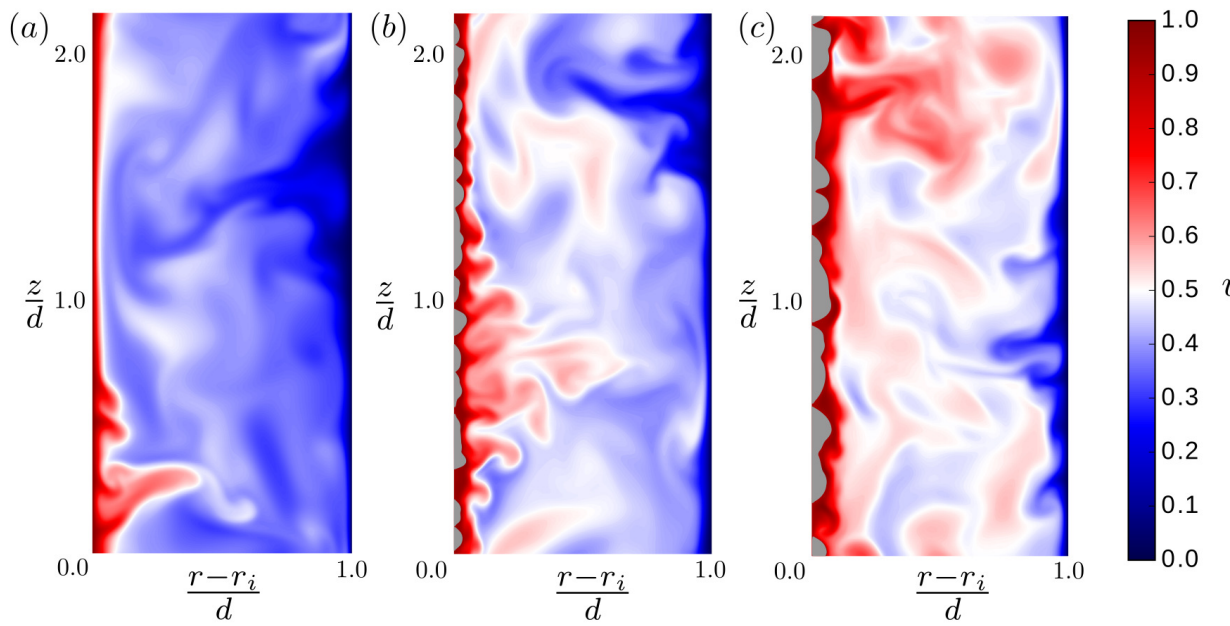

Classical turbulent state Contour fields of the instanteneous azimuthal velocity $u(r, \theta, z, t)$ for $T a=5.0 \times 10^{7}$ in the meridional plane. (a) Smooth wall simulation (BS) in which we observe one ejecting plume and one impacting plume. (b) Rough inner cylinder $k / d=0.039$ (B2), with the roughness indicated in grey, exhibiting more plumes ejecting from the inner cylinder radially outwards. (c) Rough inner cylinder $k / d=0.073$, with the roughness indicated in grey, (B4) leading to a more chaotic flow field, with enhanced mixing and enhanced radial transport of the conserved angular velocity flux.
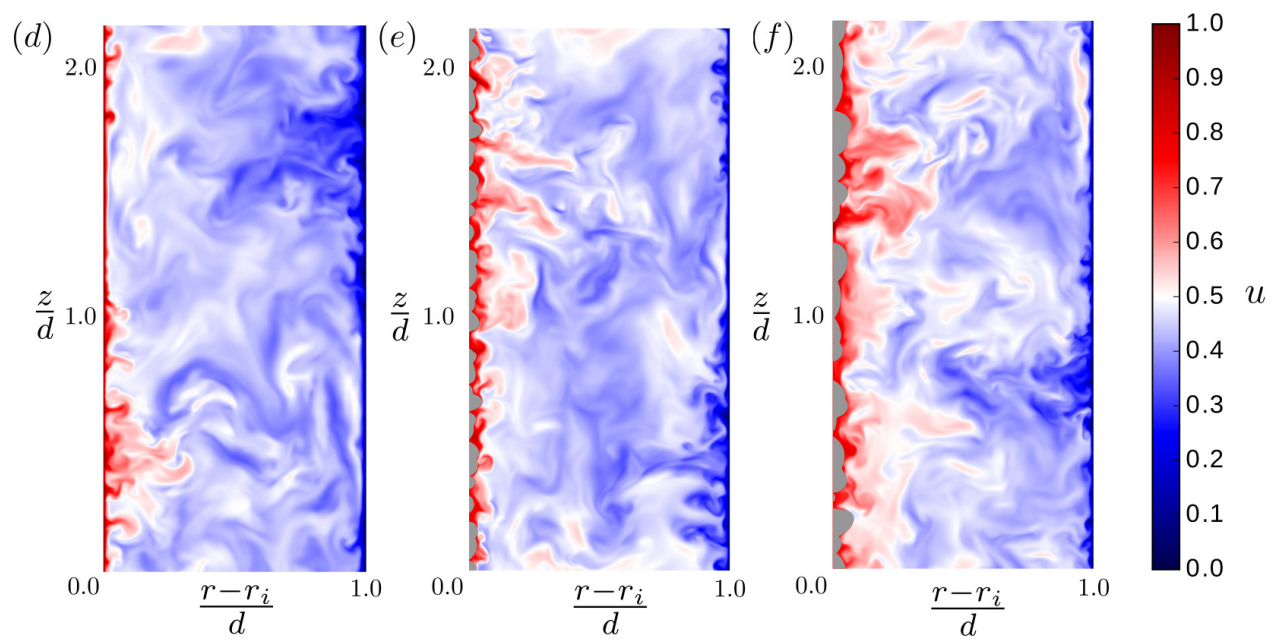

Ultimate turbulent state Contour fields of the instanteneous azimuthal velocity $u(r, \theta, z, t)$ for $T a=1.0 \times 10^{9}$ in the meridional plane. (d) Smooth inner cylinder (DS) with many plumes ejecting, considerably more chaotic than in (a). (e) Inner cylinder wall roughness (indicated in grey) $k / d=0.035$ (D2) and $(f)$ inner wall roughness $k / d=0.055$ (D5). For the rough cases, we observe more plumes ejecting and more mixing in the bulk, leading to enhanced radial transport of the angular velocity $J^{\omega}$, expressed in a higher $N u_{\omega}$. 
other hand, the boundary layers remain predominantly laminar and as such the regime is referred to as the 'classical regime' of TC turbulence. A divergent colormap is chosen to highlight the turbulent structures in the bulk. A snapshot for the smooth inner cylinder simulation is presented in figure 1.7(a). At $z / d \approx 0.3$, one observes an ejecting structure (plume) that detaches from the inner cylinder laminar boundary layer at the location of an adverse pressure gradient. Locally, where this ejecting plume detaches, the flow will be different (i.e. more chaotic) to that in the other parts of the boundary layer. As such, one also expects the local variables (e.g. skin friction, turbulence intensity) to be different. Later we will therefore employ local averaging, to investigate the spatial differences in the flow associated with this structure ( [55-57]). The ejecting and impacting (located at $z / d \approx 1.3$ ) plumes have very strong radial velocity components $w$. From the first term on the right-hand side of (1.10), $r^{3}\left(\langle w \omega\rangle_{\theta, z, t}\right)$, we then directly see that they strongly contribute to $N u_{\omega}$. This brings us to the remaining (figure $1.7 b$ and $c$ ) snapshots. Many more, small, plumes are seen to eject from the inner cylinder. The roughness there promotes the detachment of ejecting structures and in that way contributes to a higher $N u_{\omega}$. An increase in the level of turbulence, as suggested by the increased level of turbulence dissipation, is quantitatively reflected by a decrease in the Kolmogrov scale $\left(\eta=\left(\nu^{3} / \epsilon\right)^{1 / 4}\right)$, namely $\eta / d=7.1 \times 10^{-3}$ for the smooth wall case BS and $\eta / d=6.5 \times 10^{-3}$ for the highest roughness case B5. Note that the decrease in the Kolmogorov scale $\eta$ is only small, since $\eta / d \propto\left(\epsilon d^{4} / \nu^{3}\right)^{-1 / 4}$. For TC flow, the volume averaged dissipation rate $\epsilon$ is related to the angular velocity transport $N u_{\omega}$ with: $\epsilon=\nu^{3} d^{-4} \sigma^{-2}\left(N u_{\omega}-1\right) T a+\epsilon_{\text {lam }}$, where $\epsilon_{\text {lam }}$ is the laminar volume averaged dissipation rate, $d$ is the gap width of the setup and $\sigma=\left(\frac{(1+\eta) / 2}{\sqrt{\eta}}\right)^{4}$ a geometric parameter [48]. As such, we see that $\eta / d \propto N u_{\omega}^{-1 / 4}$ only.

Figure 1.7 $(d-f)$ presents snapshots of a flow in the ultimate turbulent state at $T a=1.0 \times 10^{9}[13]$. Although less pronounced than for $T a=5.0 \times 10^{7}$, we still observe distinct ejecting and impacting regions, indicating the survival of the turbulent Taylor rolls. A similar rationale as applied above, to the classical turbulence case, can also be used to explain the enhancement of the Nusselt number for rough inner cylinders in the ultimate turbulent state. In fact, we can also observe more intense plumes for the highest roughness (D5, figure $1.7 f$ ), in comparison to a lower roughness case (D2, figure 1.7e). Note that here we do not observe the stable vortex formation in between roughness elements and the associated ejection of plumes from sharp peaks, as was reported by [41] for grooved cylinders, for similar Taylor numbers and roughness heights. The 

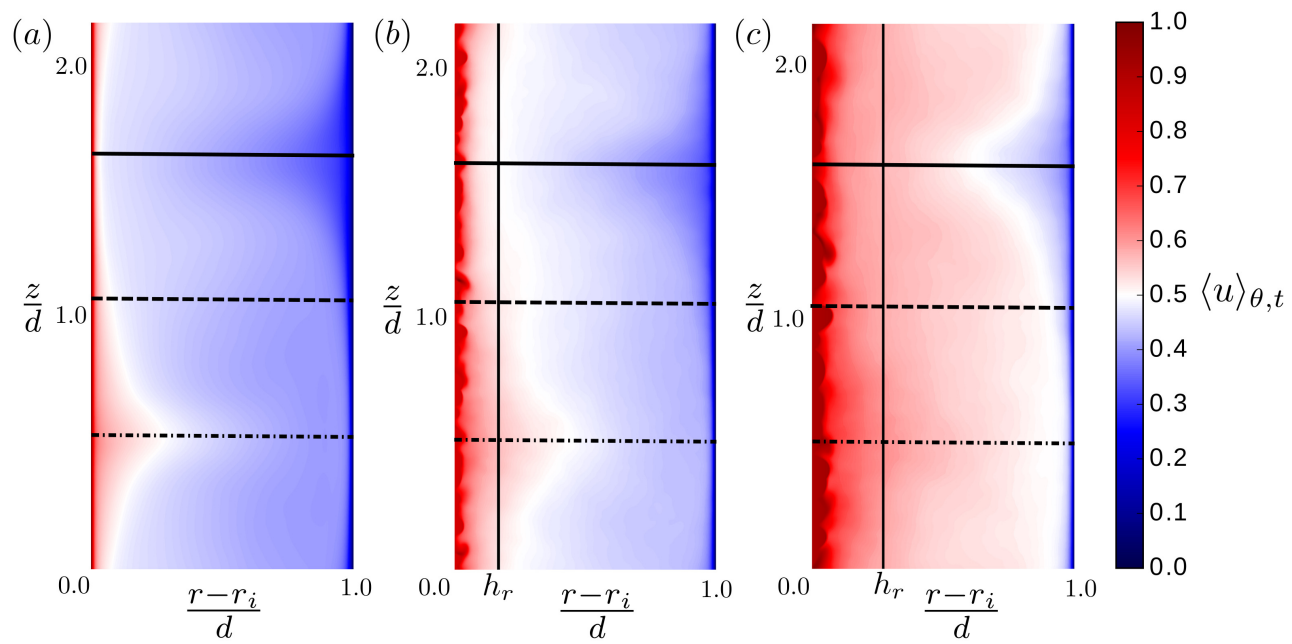

Figure 1.8: Contour field of the mean azimuthal velocity $\langle u\rangle_{\theta, t}$ in the meridional plane for $T a=1.0 \times 10^{9}$. (a) Smooth inner cylinder (DS) (b) Inner cylinder wall roughness $k / d=0.0517$ (D2) and (c) inner wall roughness $k / d=0.0818$ (D5). The solid vertical lines indicate the height of the roughness sublayer $h_{r}$, calculated over the entire cylinder height. -..... plume ejection regions, ----- sheared regions, — plume impacting regions.

increase in the turbulence level is also quantitatively confirmed by a decrease in the Kolmogorov scale here, $\eta / d=2.7 \times 10^{-3}$ for the smooth wall case DS and $\eta / d=2.1 \times 10^{-3}$ for the highest roughness case D5.

\subsubsection{Roughness sublayer}

The existence of Taylor roll structures is already anticipated in the snapshots of the instantaneous flow in figure 1.7, from which we observe the ejecting and impacting plume regions. Contour plots of the time and azimuthally averaged azimuthal velocity field, as presented in figure 1.8, confirm this. Note that the Taylor roll is spatially fixed, allowing for convenient averaging over impacting (solid line), shearing (dashed line) and ejecting (dashed dotted line) regions, a method that we also employed in RB flow [57]. For an increasing roughness height, the white region (representing $\langle u\rangle_{\theta, t} \approx 0.5$ ) shifts radially outwards and the azimuthal velocity in the bulk increases. This process previously has been seen in [43], where it is referred to as the bulk velocity 'getting slaved to' to the velocity of a cylinder covered with roughness, reflecting the enhanced 

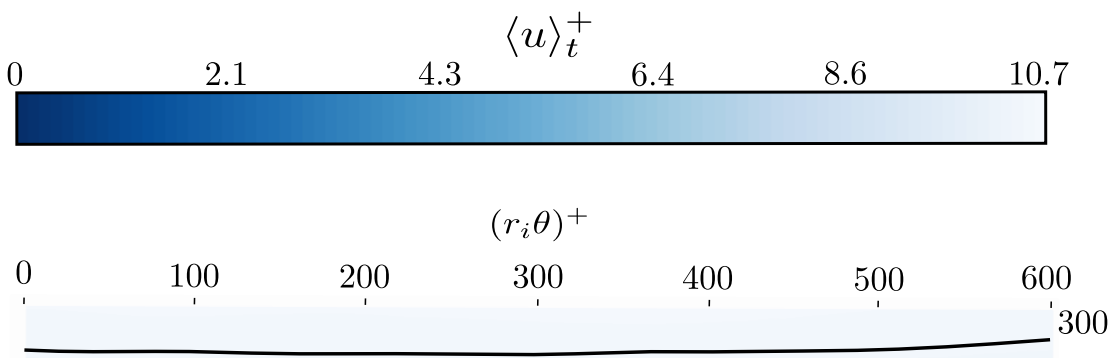

0.2

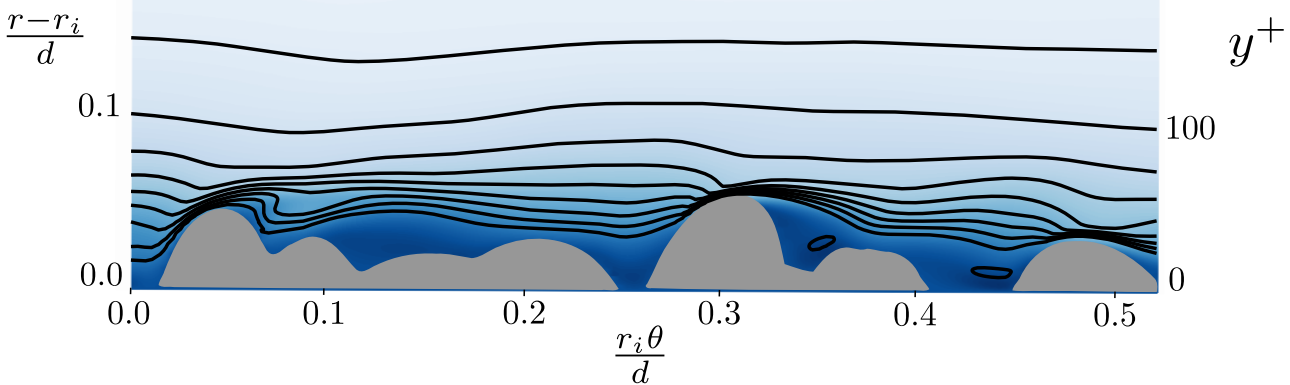

Figure 1.9: Contour of the time-averaged azimuthal velocity $\langle u\rangle_{t}^{+}$, zoomed in on only a few roughness elements, indicated in grey, for $T a=1.0 \times 10^{9}$ (D2). Flow is from left to right. Isolines of $\langle u\rangle_{t}^{+}$overlay the contour. On the vertical axis, we display the wall normal coordinate normalized by the gap width $d$ (left) and in viscous units (right). On the horizontal axis, we display the azimuthal coordinate, normalized by the gap width $d$ (below) and in viscous units (above).

drag on the rough side.

A better impression of the local fluid flow disturbances induced by the roughness elements, is obtained from the time-averaged azimuthal velocity $\langle u\rangle_{t}$, a contour of which we show in figure 1.9. We zoom in on only a few roughness elements and overlay the contour with isolines of $\langle u\rangle_{t}^{+}$. We observe that local disturbances are limited to a region of only a few times the roughness height, above which the isolines relax to approximate horizontal lines. We observe small recirculation zones (closed isolines) in open regions behind high roughness elements. To quantify the degree of roughness-induced velocity disturbances, we apply a triple decomposition to the instanteneous azimuthal 

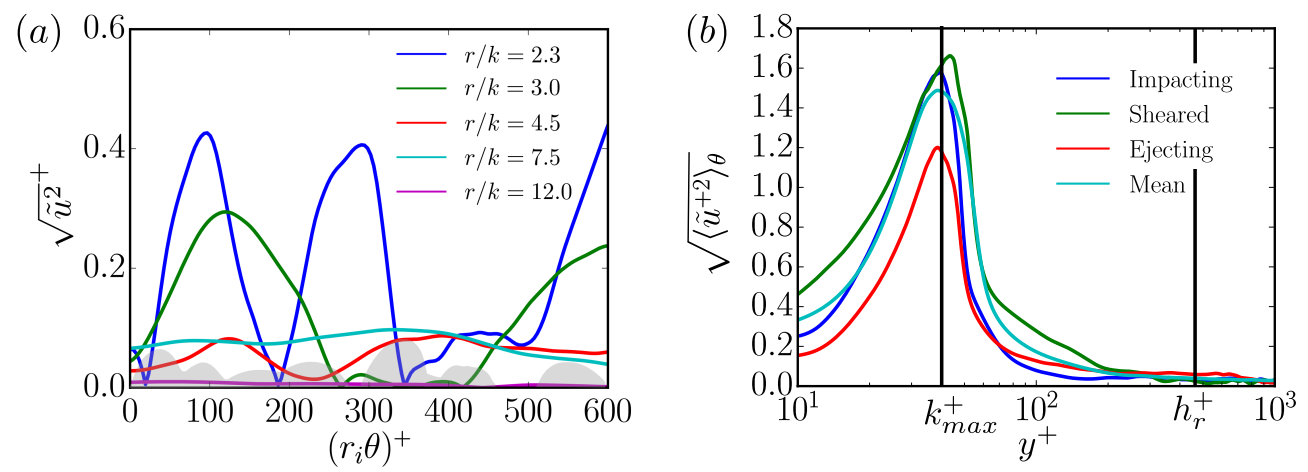

Figure 1.10: (a) Profiles of the root mean square of the form-induced azimuthal velocity fluctuations $\tilde{u}^{+}=\left(\langle u\rangle_{t}-\langle u\rangle_{\theta, t}\right) / u_{\tau}$ at incremental heights above the roughness for identical location and conditions as in figure 1.8. The radial coordinate $r^{+}$is made dimensionless by the roughness height $k^{+}$. The horizontal axis represents the azimuthal coordinate in viscous units. (b) Root means square of the azimuthally averaged form-induced velocity fluctuations. The profiles are obtained at three heights, namely where the Taylor roll impacts, ejects and in the center (sheared region) - the extent of the regions are estimated from time averaged velocity fields. The cyan line gives the mean over the entire height of the cylinder. The solid black lines indicate the maximum roughness height $k_{\text {max }}^{+}$and the height of the roughness sublayer $h_{r}^{+}$. 
velocity $u(r, \theta, z, t)[58]$ :

$$
u(r, \theta, z, t)=\langle u\rangle_{\theta, t}(r, z)+\tilde{u}(r, \theta, z)+u^{\prime}(r, \theta, z, t)
$$

where $u^{\prime}(r, \theta, z, t)=u(r, \theta, z, t)-\langle u\rangle_{t}(r, \theta, z)$, the temporal fluctuation and $\tilde{u}(r, \theta, z)=\langle u\rangle_{t}(r, \theta, z)-\langle u\rangle_{\theta, t}(r, z)$, the component that is strongly related to the roughness induced disturbances and therefore termed the form-induced (or dispersive) velocity fluctuation. Note that by applying the triple decomposition to the full NS equations and then averaging in $\theta$ and $t$, one will recover the related form-induced stress tensor $\left\langle\tilde{u}_{i} \tilde{u}_{j}\right\rangle_{\theta, t}$. However, here we only discuss $\tilde{u}(r, \theta, z)$. The root mean square of the form-induced fluctuations $\sqrt{\tilde{u}(r, \theta, z)^{2}}+$ at various heights above the roughness is given in figure 1.10(a). The horizontal axis corresponds to the roughness elements shown in figure 1.9. Already for $r / k=4.5$ (with $k$ being the roughness height, i.e. the smallest radius of the ellipsoidal building block) we find it hard to detect spatial fluctuations along $\theta$. For $r / k=12.0$, the line is barely distinguishable from the horizontal axis. If we average the lines over the azimuthal direction, we obtain the behavior of the dispersive fluctations as a function of the wall normal distance; see figure 1.10(b). We plot the lines for three respective axial locations, that is the impacting, sheared and ejecting regions, and find little variance in the wall normal extent of the form-induced fluctuations, for the varying locations. The vertical black line represent the maximum roughness height, below wich we average only over fluid regions.

Rough elements on the inner cylinder destroy the streamwise homogeneity of the flow, and thus the root mean square of the dispersive fluctuation is non-zero. Some distance from the wall, the turbulence is well able to 'mix out' the form-induced structures and the flow regains streamwise homogeneity. The distance from the wall at which the dispersive fluctuations are zero (or reasonably close to zero), is called the roughness sublayer height $h_{r}$. In this research, we define $y^{+}=h_{r}^{+}$where $\sqrt{\left\langle\tilde{u}^{+2}\right\rangle_{\theta, z}}(r)=0.01\left\langle u^{+}\right\rangle_{\theta, z, t}$ and find $h_{r}=3.70 k\left(h_{r}=2.78 k_{s}\right)$. This value agrees well with a roughness sublayer height of $2 \lesssim k_{s} \lesssim 5$, as typically found in other canonical flows [58].

The existence of a finite height of only a few times the roughness height, at which the dynamical effects of the roughness on the fluid flow vanish, is an important finding in wall-bounded turbulence. As written in [26]: '..the utility of the roughness function itself hinges on similarity of the flow'. This idea ('wall similarity') is already heavily tested in other systems $[7,26,59]$ and in the vast majority of the research found to be correct. However, in a heavily stratified system as TC, where wall-attached structures result from the unsta- 

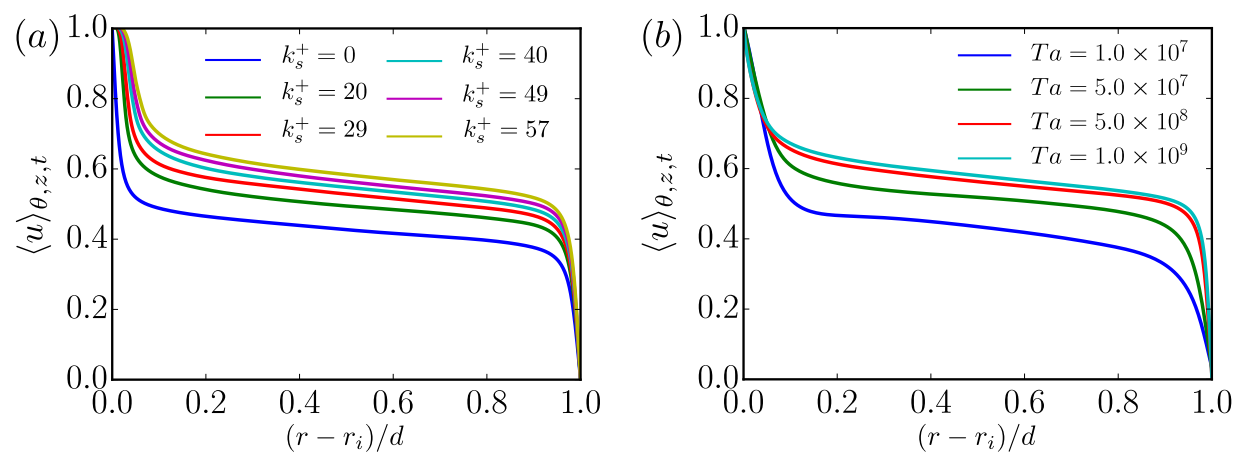

Figure 1.11: Profiles of the mean streamwise velocity $\langle u\rangle_{\theta, z, t}$ versus the radial coordinate $\left(r-r_{i}\right) / d$, where $r_{i}$ the radius of the inner cylinder and $d$ is the gap width. (a) Constant Taylor number $T a=5.0 \times 10^{8}$ and increasing roughness heights. The profiles exhibit clear 'slaving', i.e. the bulk velocity moves towards the velocity of the roughened cylinder. (b) Constant roughness height $k / d=0.060 \pm 0.002$ for increasing Taylor number. Increased slaving of the bulk velocity is observed for increasing viscous scaled roughness height $k_{s}^{+}$, respectively; (blue) $k_{s}^{+}=9$, (green) $k_{s}^{+}=24,($ red $) k_{s}^{+}=47$ and (cyan) $k_{s}^{+}=65$.

ble stratification of centrifugal pressure, the notion of wall similarity is not yet investigated. As such, the findings presented here, that such similarity exists in TC, strengthen our believe that TC could be a valued system to characterize the equivalent sandgrain roughness height of a given rough surface.

\subsubsection{Radial profiles}

The effects of roughness on a turbulent shear-driven flow, where the shear rate is constant, can be separated into two effects. The first is the change in the wall shear stress $\tau_{w}$. The second is the change in the structure of the turbulent flow at a given wall shear stress. Sand grains increase $\tau_{w}$ and therefore we find an increased momentum transfer to the bulk region with respect to a smooth wall TC case, at fixed Taylor number. This increased momentum transfer to the bulk (through plumes, very similar to Rayleigh-Bénard flow) leads to a more intense turbulence in the bulk flow, and also to higher velocity fluctuations.

We plot the time and azimuthally averaged azimuthal velocity for $T a=5.0 \times$ $10^{8}$ in figure 1.11(a). The roughness covers the inner cylinder, i.e. $\left(r-r_{i}\right) / d \leq$ 0.07. In this section we focus on the behavior of the statistics above the 

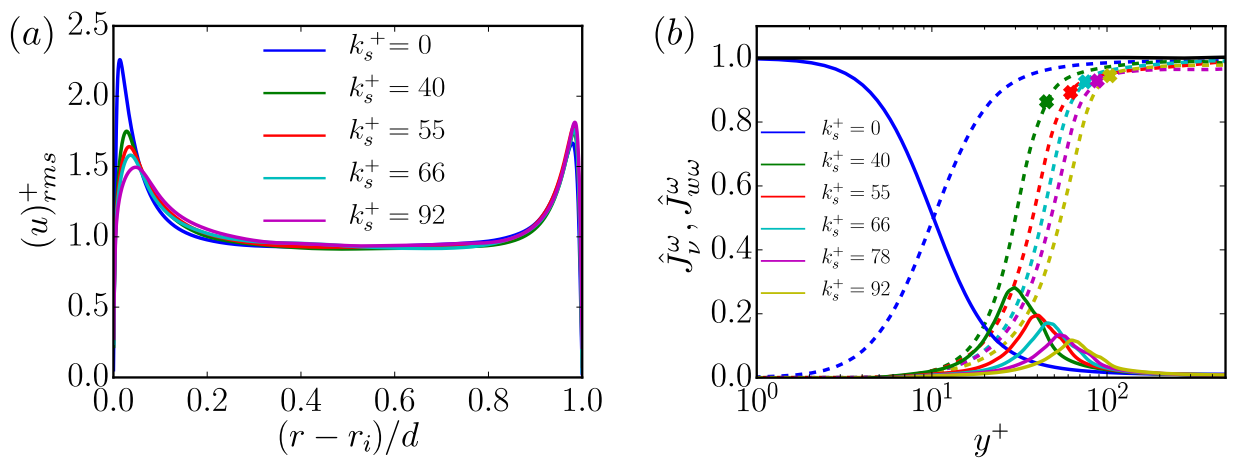

Figure 1.12: $(a)$ Root mean square of the mean streamwise velocity $(u)_{r m s}^{+}=$ $\left\langle\left\langle u^{2}\right\rangle_{\theta, z, t}-\langle u\rangle_{\theta, z, t}^{2}\right\rangle^{1 / 2} / u_{\tau}$ versus the radius $\left(r-r_{i}\right) / d$ for $T a=1.0 \times 10^{9}$. We observe an overall decrease of the viscous scaled turbulence intensity for increasing roughness height $k_{s}^{+}$. (b) The viscous $\hat{J}_{\nu}^{\omega}\left(y^{+}\right) \equiv-r^{3} \nu \partial_{r}\langle\omega\rangle_{\theta, z, t} / J_{\nu}^{\omega}\left(r_{o}\right)$ (solid line) and Reynolds stress $\hat{J}_{w \omega}^{\omega}\left(y^{+}\right) \equiv r^{3}\langle w \omega\rangle_{\theta, z, t} / J_{\nu}^{\omega}\left(r_{o}\right)$ (dashed line) terms of the conserved angular velocity flux $\hat{J}^{\omega}$ versus the wall normal distance $y^{+}$for $T a=1.0 \times 10^{9}$. The sum of the individual terms represents the total conserved angular velocity flux radially outwards. The black horizontal line at $\hat{J}^{\omega}=1.0$ is the sum of the two blue lines for the smooth wall case. It is observed that $\hat{J}^{\omega}$ is conserved above the maximum roughness height (indicated by cross markers). 
roughness. Therefore, averaging over both solid and fluid regions is carried out. For increasing roughness height, see the legend for viscous roughness heights, the azimuthal velocity in the bulk increases. Figure 1.11(b) then presents the corresponding azimuthal velocity profiles for constant $k / d=0.060 \pm 0.002$ and varying Taylor number.

Figure 1.12(a) shows the double-averaged radial profiles $(u)_{r m s}^{+}=\left\langle\left\langle u^{2}\right\rangle_{\theta, z, t}-\right.$ $\left.\langle u\rangle_{\theta, z, t}^{2}\right\rangle^{1 / 2} / u_{\tau}$ for constant Taylor number $T a=1.0 \times 10^{9}$. For the smooth wall case, the peak of $\left(u^{+}\right)_{r m s}$ is located at $y^{+} \approx 10$. This agrees with the smooth case in [55] for TC flow, but is closer to the solid boundary than is found in channel flow; $y^{+} \approx 12$. We observe a slight decrease in the viscous scaled turbulence intensity $(u)_{r m s}^{+}$for increasing roughness heights, close to the inner cylinder. Further outside the profiles, the profiles collapse.

The reason why we choose to make the variables dimensionless using their friction equivalents is to assess those changes to the flow that go beyond the effects coming from 'simply' increasing the momentum input to that region, i.e. to study the structural changes of the turbulent flow for the same momentum input. We can thus infer from figure 13(a) that the bulk of the TC flow is not affected by the roughness elements, other than increasing the momentum input into the bulk would do. A gedankenexperiment (see e.g. [59]) would make this clear. If one would be placed into the bulk of a rough TC flow, without being able to look into the boundary layer, one could not tell whether the wall is rough or smooth. The reason is that a smooth wall with a higher Taylor number can produce the same $\tau_{w}$ as a rough wall with a lower Taylor number. This idea is, in fact, Townsend's outer-layer similarity hypothesis [4]. (Note: We can only be sure that this holds for the relatively small sand grain surface under scrutiny here, owing to the sufficient scale separation!) As such, we conclude that the influence of the roughness elements pertains only to a region close to the wall.

It has already been mentioned that the angular velocity flux $J^{\omega}\left(y^{+}\right)$is conserved in the radial direction, see section 1.4.2. However, the individual components - i.e. the viscous $J_{\nu}^{\omega}\left(y^{+}\right)$and Reynolds stress $J_{w \omega}^{\omega}\left(y^{+}\right)$terms - are functions of the wall normal (radial) coordinate. The profiles of these terms are shown in figure 1.12(b). We normalize all terms with $J_{\nu}^{\omega}\left(r_{o}\right)$. The viscous stress terms are presented as solid lines and the Reynolds stress terms as dashed lines. The blue lines represent the smooth wall case. Very close to the inner cyliner $\left(y^{+}=1\right), J^{\omega} \approx J_{\nu}^{\omega}$; this is expected, since there the gradient of the mean streamwise velocity is maximum and the wall normal component of the velocity vector goes to zero. On the far right of figure 1.12(b), the 
gradient of the mean velocity approaches zero in the bulk, and the correlation function between the wall normal and angular velocity goes to a maximum; as such $J^{\omega} \approx J_{w \omega}^{\omega}$. The black line represents the sum of the viscous and Reynold stress terms, for the smooth (blue) case, and is independent of $y^{+}$. The situation for the rough cylinder cases is more complex. For increasing roughness height, the viscous stress reaches a maximum below the maximum roughness height. This can be explained by the recirculation zones behind the roughness elements. Then, above the roughness, the viscous stress goes to zero in the bulk. For the Reynolds stress terms the increase is monotonic, and similar to the smooth wall case. However, we observe a steeper increase for the rough cases. Note that under the maximum roughness height, the viscous and Reynolds stress terms do not add up to unity, since the rough surface acts as a radial dependent momentum source term to the NS equations there.

\subsection{Summary and conclusions}

We have performed Direct Numerical Simulations of turbulent Taylor-Couette flow with inner cylinder rotation and inner cylinder sand grain roughness. The Taylor number ranges from $T a=1.0 \times 10^{7}\left(R e_{\tau}=82\right)$ up to $T a=1.0 \times 10^{9}$ $\left(R e_{\tau}=635\right)$, covering thereby both the classical and the ultimate regimes of turbulent TC flow. In particular, we studied the effects of the roughness height on the fluid flow in the transitionally rough and fully rough regimes, with the equivalent sand grain roughness height ranging from $k_{s}^{+}=5$ to $k_{s}^{+}=92$. We modelled the sand grains as randomly rotated and translated ellipsoids of constant size and shape (monodisperse), similar to the model proposed by [18]. The surface was implemented into a second order finite difference code by means of the Immersed Boundary Method.

We confirm an increase in the dimensionless torque, expressed as the Nusselt number, for increasing roughness height. This is attributed to the enhanced boundary layer detachment, resulting in plume ejection regions, which we observed in snapshots of the azimuthal velocity field. The plumes contain a strong radial velocity component, and as such contribute strongly to the Reynolds stress term of the angular velocity flux. This mechanism is analogous to what was found for the $\mathrm{Nu}$ increase for grooved TC turbulence by [41].

To quantify the degree of roughness induced disturbances to the velocity field, we measured the dispersive fluctuations of the azimuthal velocity component, $\tilde{u}=\langle u\rangle_{t}-\langle u\rangle_{\theta, t}$. This dispersive term was obtained by means of a triple decomposition to the azimuthal velocity. We defined the height of the roughness 
sublayer $h_{r}$ there where the dispersive fluctuations become very small, such that $\sqrt{\left\langle\tilde{u}^{+2}\right\rangle_{\theta, z}}=0.01\left\langle u^{+}\right\rangle_{\theta, z, t}$ and found the height of the roughness sublayer to be $h_{r}=2.78 k_{s}$. This height of the roughness sublayer compares well with values found for other canonical systems [58]. The low height of the roughness sublayer in TC flow, and the existence of similarity of the flow above this sublayer, leads us to believe that we can utilize the shift of the logarithmic region to find an equivalent sand grain roughness height.

The hallmark of turbulent flows over rough walls is the shift of the logarithmic streamwise velocity profile $\Delta u^{+}$. The shift is a function of any parameters describing the roughness topology. Here we focused in particular on the effect of the sand grain size $k$ and the roughness function becomes $\Delta u^{+}\left(k^{+}\right)$. It was shown in [27] that the constants of the logarithmic law are not constant in the Taylor number range of our simulations. Hence we proposed the generalization; $u^{+}=f_{1}\left(R e_{i}\right) \log \left(y^{+}\right)+f_{2}\left(R e_{i}\right)-\Delta u^{+}$.

We performed simulations at four Ta and various roughness heights and ensured that the $k^{+}$range for the various Ta numbers overlaps. First, we concluded that the velocity shift is independent of Ta, despite the Ta dependence of the constant in the logarithmic layer. As such, all simulations collapse onto a single curve. Second, we saw a strong overlap between the roughness function calculated from our DNS in TC flow and the seminal work by [10] on monodisperse sand grains in pipe flow, in the transitionally rough regime. We found $k_{s}^{+}=1.33 k$. Only for very low $k_{s}^{+}$values, close to the hydrodynamically smooth regime, we found that the simulations slightly differ from the Nikuradse curve.

It is remarkable that the Hama roughness function appears to be universal for similar surfaces in such different systems. Note in particular that we have a streamwise curvature and strong secondary motions (Taylor rolls), which were absent in the pipe flow experiments of Nikuradse. As such, our findings point towards a universal behavior of the roughness function for very different fluid flow systems. However, many more comparison studies, of identical rough surfaces and varying fluid flow systems, are needed to confirm this notion.

\section{Appendix}




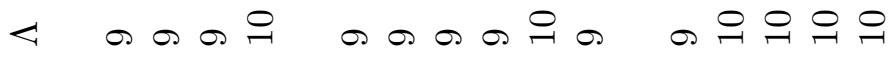

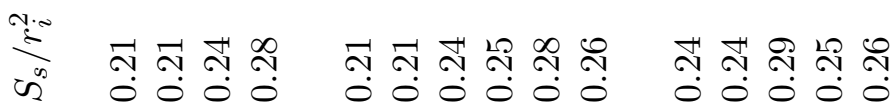

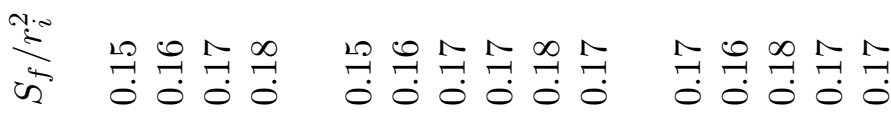

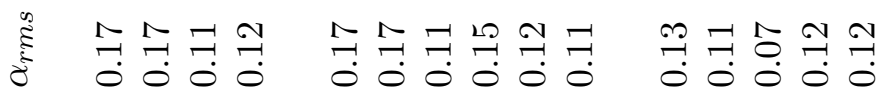

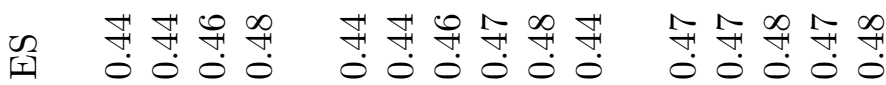

ڤ

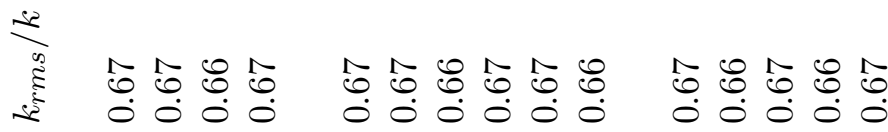

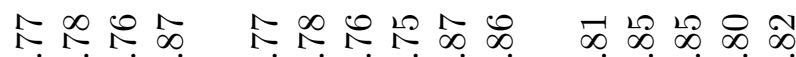

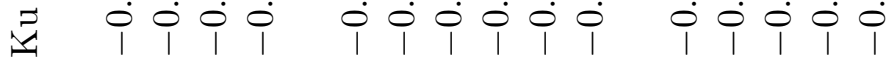

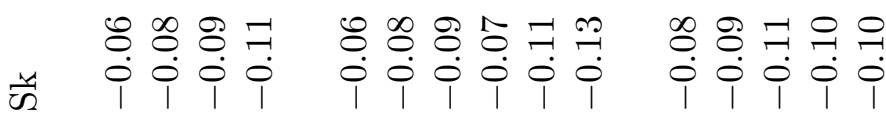

萬

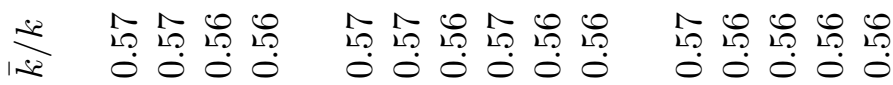

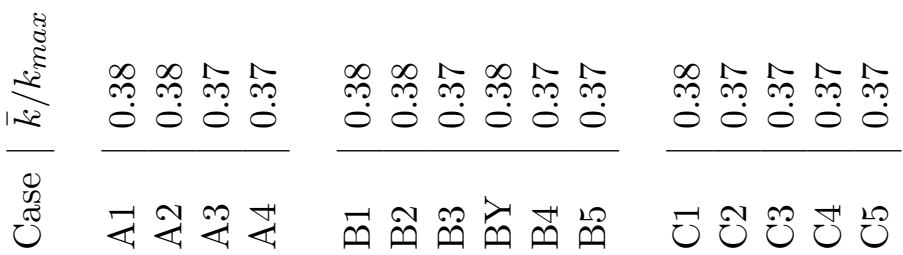




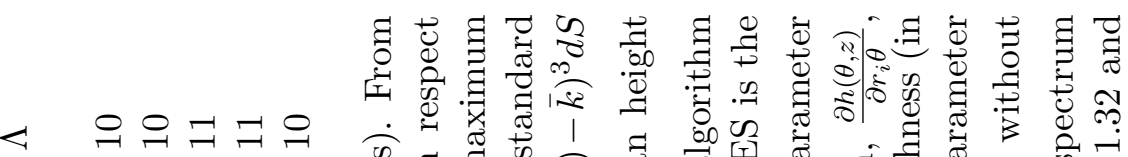

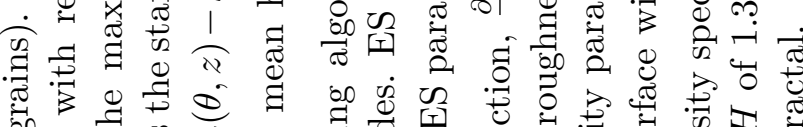
ঋ w

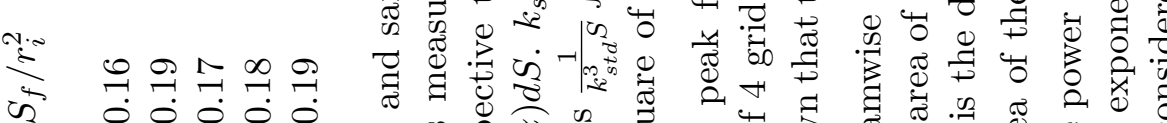
in

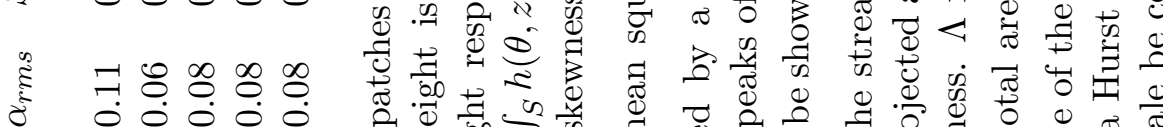
o

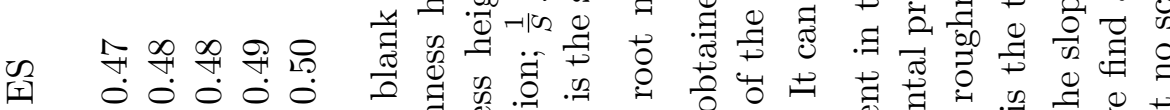

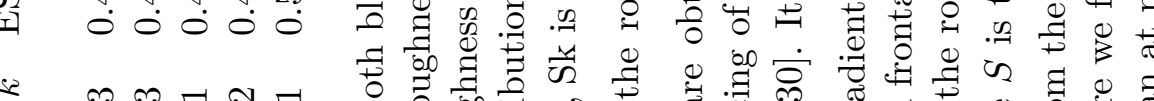
ঋ

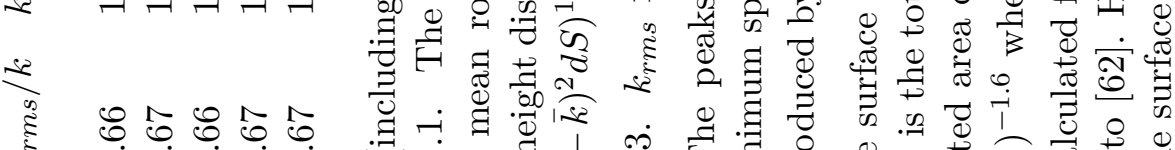

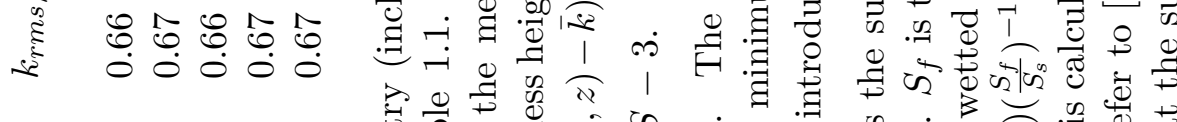

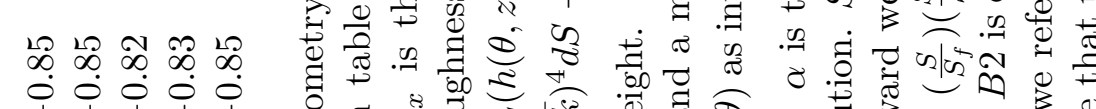

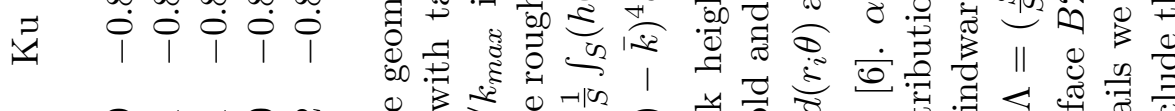

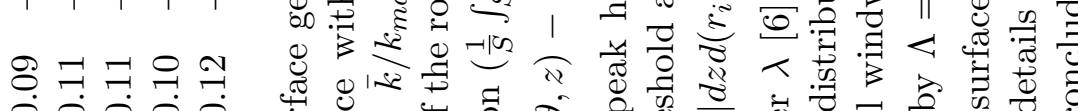

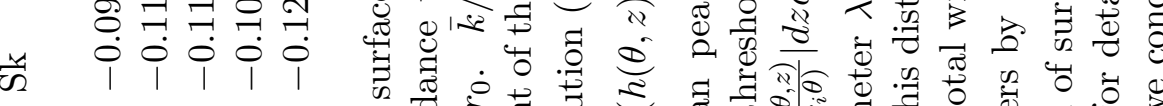

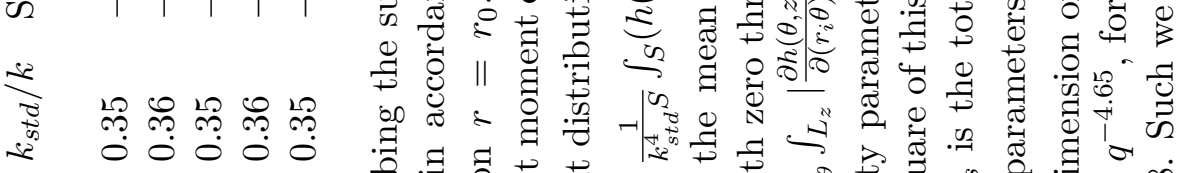

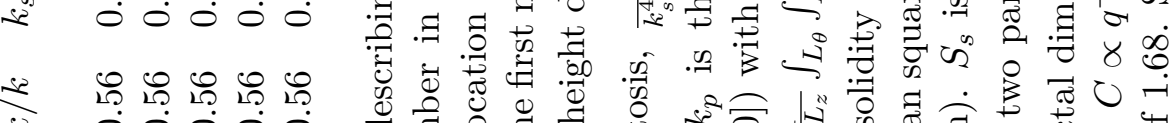

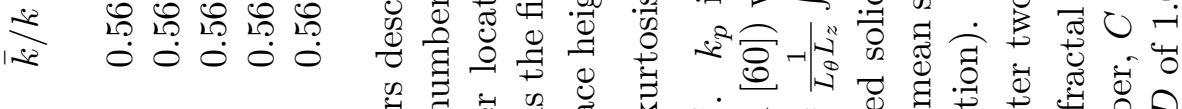

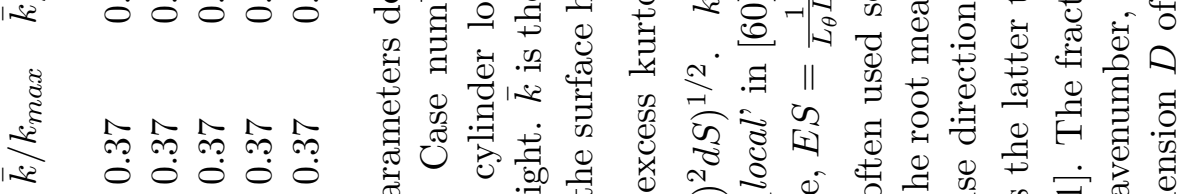

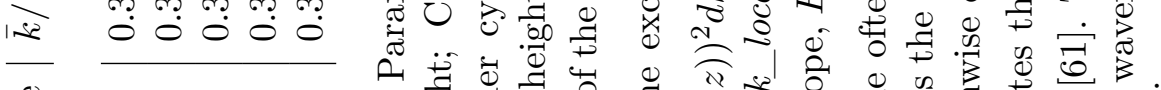
嵌

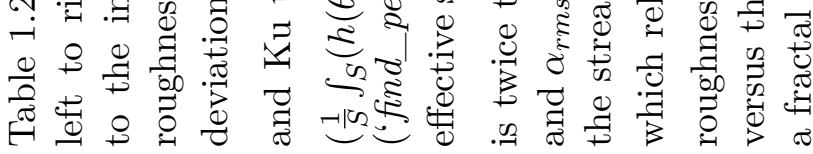




\section{Chapter 2}

\section{Calculation of the mean velocity profile for strongly turbulent Taylor-Couette flow}

Taylor-Couette (TC) flow is the shear-driven flow between two coaxial independently rotating cylinders. In recent years, high-fidelity simulations and experiments revealed the shape of the streamwise and angular velocity profiles up to very high Reynolds numbers. However, due to curvature effects, so far no theory has been able to correctly describe the turbulent streamwise velocity profile for given radius ratio, as the classical Prandtl-von Kármán logarithmic law for turbulent boundary layers over a flat surface at most fits in a limited spatial region. Here we address this deficiency by applying the idea of a Monin-Obukhov curvature length to turbulent TC flow. This length separates the flow regions where the production of turbulent kinetic energy is governed by pure shear from that where it acts in combination with the curvature of the streamlines. We demonstrate that for all Reynolds numbers and radius ratios, the mean streamwise and angular velocity profiles collapse according to this separation. We then develop the functional form of the velocity profile. Finally, using the newly developed angular velocity profiles, we show that these lead to an alternative contant in the model proposed by [15] for the dependence of the torque on the Reynolds number, or, in other words, of the generalized Nusselt number (i.e., the dimensionless angular velocity transport) on the Taylor number.

Published as: P. Berghout, R. Verzicco, R. J. A. M. Stevens, D. Lohse and D. Chung, Calculation of the mean velocity profile for strongly turbulent Taylor-Couette flow, J. Fluid Mech. 905, A11 (2020). 


\section{$2.1 \quad$ Introduction}

Most flows in nature and engineering are bounded by solid walls. In general, the flow in the immediate vicinity - at a molecular scale distance - from the wall has the velocity of the wall, the so-called no-slip boundary condition. As a consequence, a steep gradient in the mean streamwise velocity profiles exists within the boundary layer $(\mathrm{BL})$ region between the wall and the freely flowing fluid above. In the BL, the action of viscosity against the gradient of the streamwise velocity results in viscous dissipation, the conversion of kinetic energy into heat.

\subsubsection{Turbulent flow over a flat plate: Prandtl-von Kárman BL theory}

For slowly flowing fluids (low Reynolds numbers), the edge of the BL remains smooth, and the fluid flow in the BL is two-dimensional. This laminar BL is described by the famous Prandtl-Blasius self-similar solution [63]. However, for fast flowing fluids (high Reynolds numbers), the BL becomes turbulent, and the flow inside the BL becomes vortical and three-dimensional. Although exact solutions of these turbulent BLs do not exist, a well-established functional form of the mean streamwise velocity can be obtained based on simple dimensional arguments [63]. The hallmark result therefrom can be obtained from realizing that the mean streamwise velocity gradient in the wall-normal direction $\left(\frac{d u}{d y}\right)$ is a function of two dimensionless parameters only [11],

$$
\frac{d u}{d y}=\frac{u_{\tau}}{y} \Phi\left(\frac{y}{\delta_{\nu}}, \frac{y}{\delta}\right)
$$

where $u_{\tau}$ is the friction velocity defined as $u_{\tau}=\sqrt{\tau_{w} / \rho}, \tau_{w}$ is the mean wall shear stress, $\rho$ is the fluid density, $\delta$ is the outer length scale (e.g. the BL thickness), and $\delta_{\nu}$ is the viscous length scale $\delta_{\nu}=\nu / u_{\tau}$, with $\nu$ the kinematic viscosity of the fluid. Non-dimensionalization by the viscous scales $u_{\tau}$ and $\delta_{\nu}$ is indicated by a superscript ' + '. The friction Reynolds number based on these viscous quantities is $R e_{\tau}=\frac{u_{\tau} \delta}{\nu}=\frac{\delta}{\delta_{\nu}}$, and $u_{\tau}=u_{\tau, i}$, where the subscript $i$ refers to the inner cylinder. For TC turbulence $\delta=d / 2$, with $d$ the gap width between the two rotating cylinders. If we assume that the dependence of the gradient of the mean velocity on viscosity vanishes with increasing $R e_{\tau}$, the yet undefined function $\Phi\left(\frac{y}{\delta_{\nu}}, \frac{y}{\delta}\right)$ must go to a constant $\left(=\kappa^{-1}\right)$ when $\delta_{\nu} \ll y \ll \delta$, which is known as the inertial sublayer. In this limit, we can integrate (2.1) 
and arrive at the celebrated logarithmic law of the wall for turbulent BLs over a flat surface:

$$
u^{+}=\kappa^{-1} \log y^{+}+B .
$$

This law is connected with the names of Prandtl and von Kármán. It is supported by overwhelming experimental and numerical evidence (e.g. [64]). The values of the two parameters are $\kappa \approx 0.39$ and $B \approx 5.0$.

An important extension of the theory concerns buoyancy stratified BLs, where an additional forcing acts on the wall-normal momentum component. A prominent example of such a system is the atmospheric surface layer, where thermal forcing stabilizes or destabilizes the flow. The thermal stratification introduces, aside from $\delta_{\nu}$ and $\delta$, a third relevant length scale: the Obukhov length $L_{o b}$ (introduced in the year $1946 \mathrm{cf}$. [65]). This length $L_{o b}$ is proportional to the distance from the wall above which the production of turbulence is significantly affected by buoyancy, and below which the production of turbulence is governed purely by shear. With the introduction of this length $L_{o b},(2.1)$ becomes:

$$
\frac{d u}{d y}=\frac{u_{\tau}}{y} \Phi\left(\frac{y}{\delta_{\nu}}, \frac{y}{\delta}, \frac{y}{L_{o b}}\right),
$$

which was first proposed by [66]. For the inertial sublayer viscous effects and the domain size effects are negligible $\left(\delta_{\nu} \ll y \ll \delta\right)$ and only the dependence on $\frac{y}{L_{o b}}$ remains. Various empirical fits exist for $\Phi\left(\frac{y}{L_{o b}}\right)$. Evidently, in the limit of $\frac{y}{L_{o b}} \ll 1$ they must obey $\Phi\left(\frac{y}{L_{o b}}\right)=\kappa^{-1}$, thus indicating that buoyancy plays no role. We point to section 4 of [1] for an in-depth analysis of stratified BLs.

\subsubsection{Turbulent flow with streamwise curvature: Taylor-Couette turbulence}

Whereas flat plate BLs are often studied, and the existence of a logarithmic profile of the mean streamwise velocity is well established, the study of flows with streamwise curvature is less developed, despite its ubiquity, e.g. ship hulls or turbomachinery. In this chapter, we attempt to narrow this gap. One canonical system for flow in a curved geometry is Taylor-Couette (TC) flow. TC flow is the shear-driven flow in between two coaxial, independently rotating cylinders. Since the physical system is closed, one can derive a global balance between the differential rotation of the cylinders and the total energy dissipation in the flow, which is directly related to the torque $(T)$ on any of the cylinders [13].

The dimensionless torque $G$ is defined as $G \equiv T /\left(\rho \nu^{2} L_{z}\right)$, where $L_{z}$ is the height of the cylinder. It depends on the Reynolds number of the inner and 
outer cylinder, defined as $R e_{i, o}=\omega_{i, o} r_{i, o} d / \nu$. Here, $r_{i, o}$ is the radius of the inner (outer) cylinder and $\omega_{i, o}$ is the angular velocity of the inner (outer) cylinder. The relation $G\left(R e_{i}, R e_{o}, \eta\right)$ is directly connected to the structure of the mean velocity profile. Uncovering this relation - for its fundamental implications and practical relevance - can be considered the primary research question.

In this chapter we consider pure inner cylinder rotation (i.e. outer cylinder Reynolds number is zero $R e_{o}=0$ ), for which in the laminar case [12] derived that $G \propto R e$. For intermediate $R e,[67]$ - in analogy to the work of [68] on Rayleigh-Bénard (RB) flow - argued by exploring marginal stability arguments that $G \propto R e^{5 / 3}$. He modelled the flow domain as being partitioned into a turbulent bulk region with constant angular momentum L [4] and two laminar BLs. For high but finite Re, the BLs become turbulent $[55,69,70]$, and the effective scaling exponent increases with increasing $R e[49,71]$. Analogous to the interpretation of the strongly turbulent regime by [72] and [73] in RB flow, [74] derived logarithmic corrections to the $G(R e)$ scaling, coming from the turbulent BLs, such that $G \propto R e^{2} \times \log (\mathrm{Re})$-corrections. Recently, [15] obtained an accurate calculation of the torque by matching the BL and bulk velocity profiles (here referred to as the CPS model).

High-fidelity data on the structure of the BL are essential for testing all proposed scaling relationships. Therefore, much work has been carried out to determine the mean streamwise velocity profile at high Re. [75] used particle image velocimetry (PIV) and laser doppler velocimetry (LDA) to study the turbulent BL at an unprecedented resolution. For $\eta=0.716$, where $\eta$ is the radius ratio, they find that for high $R e_{i}$, i.e. $R e_{i}=O\left(10^{6}\right)$, the classical logarithmic BL exists only in a very limited spatial region of $50<y^{+}<600$. [76] employed PIV to study the velocity profiles at low radius ratio of $\eta=0.50$, for which the curvature effects are stronger, and find no von Kármán type logarithmic BL. For $\eta=0.91$, [45] and [55] employed direct numerical simulations (DNSs) and find that the slope of the mean streamwise velocity profile is ever changing with $R e_{i}$, at least up to $R e_{i}=O\left(10^{5}\right)$. We further note that [77] argue that the appropriate velocity that obeys the classical von Kármán profile is the angular velocity, rather than the streamwise velocity, based on conservation laws of the Navier-Stokes equations in this axial symmetry.

In this chapter we will explain that the introduction of a curvature length scale delineates the region where one can expect a shear-dominated turbulent BL and another region where curvature effects will alter the structure of the flow, similar as the Obukhov length in stratified shear flow separates the 
shear dominated regime from the buoyancy dominated regime. This chapter is organized as follows: In section 2 we will give the Navier-Stokes equations and boundary conditions for TC flow. In section 3 we will discuss the used datasets. We will then, in section 2.4, derive a functional form for the angular velocity throughout the entire BL for arbitrary Reynolds numbers but only for pure inner cylinder (IC) rotation. We extend the theory towards varying radius ratios in section 2.5 . Finally, we match the $\mathrm{BL}$ and bulk velocity profiles and arrive at a new functional form for $\mathrm{Nu}(\mathrm{Ta})$ and $\mathrm{C}_{\mathrm{f}}\left(\mathrm{Re}_{i}\right)$ for $\mathrm{TC}$ in section 2.6. The chapter ends with conclusions and an outlook.

\subsection{Navier-Stokes equations for Taylor-Couette flow}

When the inner cylinder rotates and the outer cylinder (OC) remains stationary (the case to which we restrict in this chapter), TC flow is linearly unstable [78]. The ratio between the destabilizing centrifugal force and the stabilizing viscous force is expressed by the Taylor number [12],

$$
\mathrm{Ta}=\frac{(1+\eta)^{4}}{64 \eta^{2}} \frac{\left(r_{o}-r_{i}\right)^{2}\left(r_{i}+r_{o}\right)^{2}\left(\omega_{i}-\omega_{o}\right)^{2}}{\nu^{2}} .
$$

The Reynolds number $\operatorname{Re}_{i, o}$ is related to Ta via the relation $\operatorname{Re}_{i}-\eta \operatorname{Re}_{o}=\frac{\mathrm{Ta}^{1 / 2}}{f(\eta)}$ with $f(\eta)=\frac{(1+\eta)^{3}}{8 \eta^{2}}$. [25] showed that the mean angular velocity flux

$$
J^{\omega}=r^{3}\left[\left\langle u_{r} \omega\right\rangle_{A(r), t}-\nu \partial_{r}\langle\omega\rangle_{A(r), t}\right]
$$

is independent of $r$, where $\langle\cdot\rangle_{A(r), t}$ refers to averaging over a cylindrical surface $A(r)$ and time $t$. The torque $T$ per unit length is related to $J^{\omega}$ by $T=2 \pi \rho J^{\omega}$. Therefore also $T$ is constant with $r$.

TC flow, see the schematic in figure 2.1, is described by the three components of the Navier-Stokes equations in an inertial frame in cylindrical coordinates as [79], with $w_{r}$ the radial velocity, $u_{\theta}$ the azimuthal velocity and $v_{z}$ the axial velocity

$$
\begin{gathered}
\partial_{t} w_{r}+(\mathbf{u} \cdot \nabla) w_{r}-\frac{u_{\theta}^{2}}{r}=-\partial_{r} P_{t}+\nu\left\{\triangle w_{r}-\frac{2}{r^{2}} \partial_{\theta} u_{\theta}-\frac{w_{r}}{r^{2}}\right\}, \\
\partial_{t} u_{\theta}+(\mathbf{u} \cdot \nabla) u_{\theta}+\frac{w_{r} u_{\theta}}{r}=-\frac{1}{r} \partial_{\theta} P_{t}+\nu\left\{\triangle u_{\theta}+\frac{2}{r^{2}} \partial_{\theta} w_{r}-\frac{u_{\theta}}{r^{2}}\right\}, \\
\partial_{t} v_{z}+(\mathbf{u} \cdot \nabla) v_{z}=-\partial_{z} P_{t}+\nu \triangle v_{z},
\end{gathered}
$$




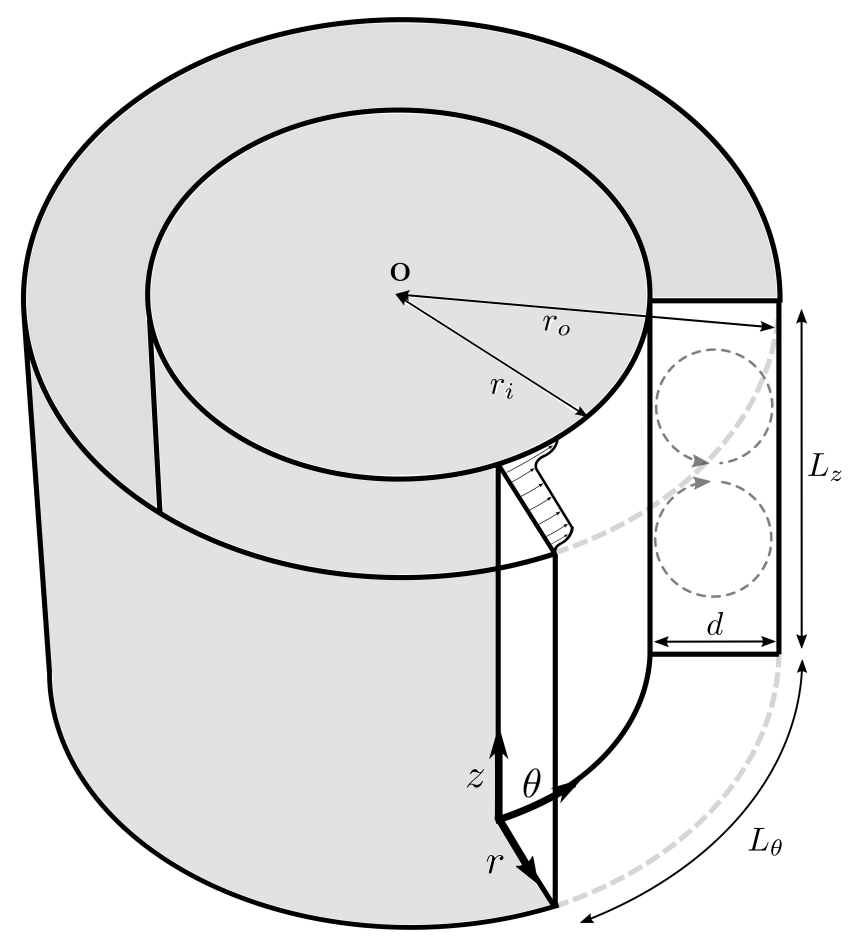

Figure 2.1: Schematic of TC flow including the coordinate directions $(\theta, z, r)$, IC radius $r_{i}$, OC radius $r_{o}$, gap width $d$, the spanwise (axial) extent of the flow domain $L_{z}$, and the streamwise extent of the flow domain $L_{\theta}$, which is used in DNSs that employ periodic boundary conditions in the azimuthal directions. $\eta=r_{i} / r_{o}$ is the radius ratio. The grey dashed circular arrows represent the turbulent Taylor vortices. 
where the operators are,

$$
(\mathbf{u} \cdot \nabla) f=w_{r} \partial_{r} f+\frac{u_{\theta}}{r} \partial_{\theta} f+v_{z} \partial_{z} f
$$

and

$$
\triangle f=\frac{1}{r} \partial_{r}\left(r \partial_{r} f\right)+\frac{1}{r^{2}} \partial_{\theta}^{2} f+\partial_{z}^{2} f,
$$

with for IC rotation only, the boundary conditions $w_{r}\left(r_{i}\right)=w_{r}\left(r_{o}\right)=0$, $v_{z}\left(r_{i}\right)=v_{z}\left(r_{o}\right)=0, u_{\theta}\left(r_{i}\right)=r_{i} \omega_{i}$ and $u_{\theta}\left(r_{o}\right)=r_{o} \omega_{o}=0$. Note that $P_{t}$ is the kinematic pressure, and $\rho P_{t}$ is the physical pressure. The continuity equation reads

$$
\frac{1}{r} \partial_{r}\left(r w_{r}\right)+\frac{1}{r} \partial_{\theta} u_{\theta}+\partial_{z} v_{z}=0 .
$$

\subsection{Employed datasets}

In this chapter we apply our analysis to published datasets with varying radius ratio, see Table 2.1 in the appendix. We now briefly describe the techniques that are used to acquire these datasets. However, we refer to the original papers for more details.

[75] did experiments on highly turbulent inner cylinder rotating TC flow with the Twente turbulent TC facility $\left(T^{3} C\right)$ [80], with the radius ratio $\eta=0.716$ and the aspect ratio $\Gamma=11.7$. In particular, they carried out PIV and particle tracking velocimetry (PTV) to measure the mean and the variance of the streamwise velocity profiles at $9.9 \times 10^{8} \leq \mathrm{Ta} \leq 6.2 \times 10^{12}$, for both the IC $\mathrm{BL}$ and the OC BL.

[76] performed experiments on turbulent TC flow in the classical turbulent regime (i.e., before the BLs become turbulent) with the Cottbus TC facility [81], with radius ratio $\eta=0.50$ and aspect ratio $\Gamma=20$. They carried out PIV to measure the mean streamwise and wall-normal velocity profiles at $5.8 \times 10^{7} \leq \mathrm{Ta} \leq 6.2 \times 10^{9}$. Although [76] carried out both counter rotation and pure IC rotation experiments, we will discuss here the latter dataset only.

[55] carried out DNSs of highly turbulent IC rotating TC flow by using a second-order finite-difference scheme [50,51]. With a radius ratio of $\eta=0.909$ they simulated three cases with $1.1 \times 10^{10} \leq \mathrm{Ta} \leq 1.0 \times 10^{11}$. Additionally, they simulated a large gap case, $\eta=0.5$, with $\mathrm{Ta}=1.1 \times 10^{11}$. For all cases the aspect ratio was fixed at $\Gamma=2 \pi / 3$. We refer to [46] who found that the aspect ratios of the numerical simulations are sufficiently large to obtain the correct velocity profiles. 


\subsection{Velocity profiles in Taylor-Couette turbulence}

Whereas effects of spanwise curvature on the velocity profiles in pipe flow were investigated before [82], in this section we set out to develop a new functional form of the mean angular velocity profile $\omega^{+}\left(y^{+}\right)$(with $\omega^{+}=\omega / \omega_{\tau}$, $\omega_{\tau,(i, o)}=u_{\tau,(i, o)} / r_{(i, o)}, \omega=\omega_{i}-u_{\theta} / r$ for the IC BL and $\omega=u_{\theta} / r$ for the OC $\mathrm{BL}$ ) in that part of the IC BL and $\mathrm{OC} \mathrm{BL}$ where the streamwise curvature effects are significant. Note that (2.1) can also be postulated for $\omega(y)$, so that the gradient becomes

$$
\frac{d \omega}{d y}=\frac{\omega_{\tau}}{y} \Phi_{\omega}\left(\frac{y}{\delta_{\nu}}, \frac{y}{\delta}\right)
$$

where $\Phi_{\omega}\left(\frac{y}{\delta_{\nu}}, \frac{y}{\delta}\right)$ goes to a constant in the inertial region $\delta_{\nu} \ll y \ll \delta$. We follow the conclusion of [77], namely that near the wall the angular velocity $\omega^{+}\left(y^{+}\right)$fits to a logarithmic form closer than the azimuthal velocity $u^{+}\left(y^{+}\right)$, and we apply our analysis to $\omega^{+}\left(y^{+}\right)$. For reference we have added figure 2.12 in the appendix, where we apply the analysis (see following pages) to the azimuthal velocity profile.

In section 2.4.1 we first derive the curvature Obukhov length and then apply our analysis to the highest Re dataset available [75]. Subsequently, we analyse both the IC BL (section 2.4.2) and OC BL (section 2.4.4) and in section 2.4.3 also the constant angular momentum region in the bulk.

\subsubsection{Derivation of the curvature Obukhov length $L_{c}$}

Following [14], we draw the analogy between the effects of buoyancy and streamline curvature on turbulent shear flow. Therefore it is informative to assess the balance of turbulent kinetic energy (TKE) in the flow. To do so, we first Reynolds-decompose the velocity and pressure field ((2.6) to (2.8)), such that $\mathbf{v}=\mathbf{U}+\mathbf{u}$, where $\mathbf{v}=\left(w_{r}, u_{\theta}, v_{z}\right)$ is the full velocity, $\mathbf{U}=(W, U, V)$ is the time averaged velocity and $\mathbf{u}=(w, u, v)$ is the fluctuating component. Upon multiplying the decomposed Navier-Stokes equations by $\mathbf{u}$, and then taking the time average, we arrive at the TKE equations. In vector notation, with the definition of TKE (per unit mass) being $q=\frac{1}{2}\left(\overline{u^{2}}+\overline{v^{2}}+\overline{w^{2}}\right)$, the 
(a)

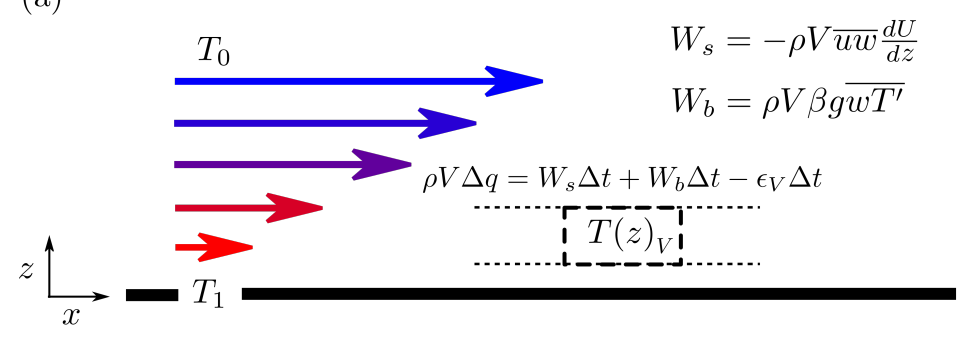

(b)

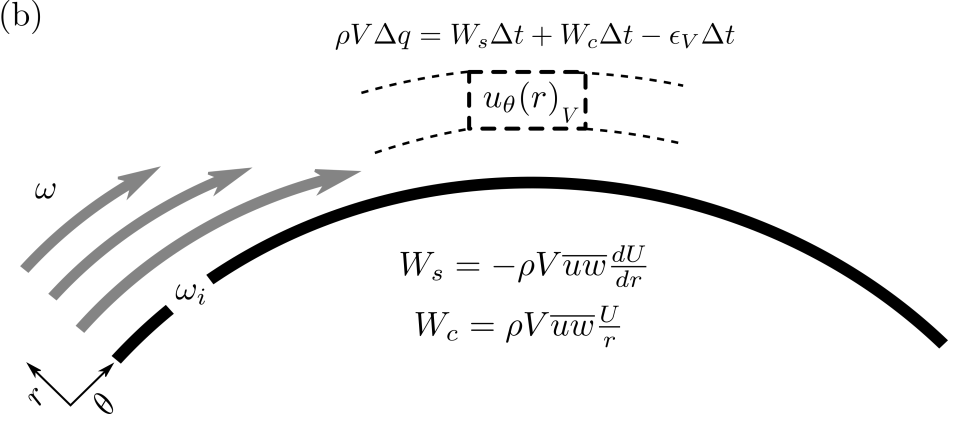

Figure 2.2: A schematic representation of the analogy between the effects of buoyancy and streamline curvature on a BL. (a) A flat plate unstably stratified $\mathrm{BL}$. The change in energy production is governed by the work carried out on a volume element $V$ by buoyancy $W_{b}$ and shear $W_{s} . \beta$ is the thermal expansion coefficient, $g$ is the gravitational acceleration that is defined positive in the $-z$ direction, $T^{\prime}$ is the temperate fluctuation and $\epsilon_{V}$ is the volumetric dissipation rate. (b) A side view of a BL over a curved surface (or the top view of TC IC). In analogy to positive work carried out by buoyancy fluctuations in an unstably stratified thermal BL (a), the rate of work done by centrifugal forces $W_{c}$ in the case of IC rotation is also positive. 
TKE equation reads (see also [83]):

$$
\begin{gathered}
\partial_{t} q+\nabla \cdot(q \mathbf{U})+\frac{1}{2} \nabla \cdot \overline{\mathbf{u}(\mathbf{u} \cdot \mathbf{u})}=-\nabla \cdot \overline{p \mathbf{u}}-\frac{\overline{p w}}{r}-\overline{\mathbf{u u}}: \nabla \mathbf{U} \\
-\frac{1}{2 r}\{2 W q+\overline{w(\mathbf{u} \cdot \mathbf{u})}\}-\frac{2 \bar{u}^{2} W}{2 r}+\overline{u w} \frac{U}{r}-\nu \overline{\nabla \mathbf{u}: \nabla \mathbf{u}} \\
+\nu\left\{\triangle q-\frac{\left(\overline{u^{2}}+\overline{w^{2}}\right)}{r^{2}}+\frac{2}{r^{2}}\left(\overline{u \partial_{\theta} w}-\overline{w \partial_{\theta} u}\right)\right\},
\end{gathered}
$$

where : is the double dot product. We consider a statistically stationary flow that is homogeneous in the wall-parallel directions. Further, we assume that the net radial transport of TKE over the boundaries of a thin cylindrical cell in the turbulent $\mathrm{BL}$ is zero for $\delta_{\nu} \ll y \ll \delta$. We then arrive at a reduced form of (2.13), where the net local production of TKE is equal to the local dissipation $\epsilon$ [11].

$$
\overline{u w} \partial_{r} U-\frac{1}{r} \overline{u w} U=-\epsilon,
$$

The first term on the left-hand-side of equation (2.14) represents the production of TKE due to a gradient of the mean streamwise velocity profile, i.e. shear. The curvilinear coordinate system gives rise to an additional production term (the second term), as compared to turbulent shear flow over a flat boundary. In fact, such additional production terms due to curvature appear both in the $u_{\theta}$-component equation and in the $w_{r}$-component equation, and are respectively, $\frac{1}{r} \overline{u w} U$ and $-\frac{2}{r} \overline{u w} U$. Together, they sum up to the second term on the left-hand-side in (2.14).

The process of additional production of TKE by curvature of the streamlines may be explained by the conservation of angular momentum $\mathrm{L}=\operatorname{Ur}[4,78]$. If one considers a vortex that exchanges two fluid elements from $r_{1}$ to $r_{2}$ where $r_{1}<r_{2}$ so that the vorticity vector points in the streamwise direction, e.g. the Taylor vortex, the change in kinetic energy whilst conserving $\mathrm{L}$ is $\Delta E_{k}=\frac{1}{2}\left(U_{2}^{2} r_{2}^{2}-U_{1}^{2} r_{1}^{2}\right)\left(\frac{1}{r_{1}^{2}}-\frac{1}{r_{2}^{2}}\right)$. For $\left(r_{2}-r_{1}\right) / r_{1} \ll 1$, the change in $E_{k}$ can be rewritten as

$$
\delta E_{k}=\frac{1}{r^{3}} \frac{d \mathrm{~L}^{2}}{d r}(\delta r)^{2}
$$

where $\delta r \approx r_{2}-r_{1}$ and $r \approx r_{1} \approx r_{2}$. This is a very similar energy exchange as for buoyancy stratified flows, where $\delta E_{k}=\beta g \frac{d T}{d z}(\delta z)^{2}$ [84]. In fact, we see that if $d \mathrm{~L}^{2} / d r<0$, the work carried out by the vortex is negative and the IC rotating and stationary OC TC flow might be called unstably stratified $[78,85]$, 
whereas for $d \mathrm{~L}^{2} / d r>0$ (OC rotating, IC stationary) the work carried out by the vortex is positive and the flow is stably stratified.

In pursuing this analogy, which we illustrate in figure 2.2 , we expect a region in the flow where $\left(\partial_{r} U \gg U / r\right)$ from (2.14) such that the production of TKE is governed solely by shear, and the flow there behaves identical to flat plate BLs. Next to this, another region might exist where the production of TKE is governed solely by curvature effects $\left(U / r \gg \partial_{r} U\right)$ and curvature stratification effects dominate. The demarcation line that separates the two regions is the location where both mechanisms are of comparable magnitude. [14] recognized the similarity between buoyancy effects and streamline curvature, and defined the curvature analogy of the Obukhov length, here called $L_{c}$, with

$$
L_{c}=\frac{\overline{u w} \partial_{r} U}{\frac{1}{r} \overline{u w} U} y,
$$

where $y=r-r_{i}$. Hence, the curvature Obukhov length $L_{c}(y)$ is the distance from the wall $(y)$ where production of turbulence by shear and curvature balance. We realize that $\overline{u w} \approx u_{\tau}^{2}$ and the gradient of the streamwise velocity in the shear dominated region is $\partial_{r} U=\frac{u_{\tau}}{\kappa y}$, see (2.1), which we take for reference in defining $L_{c}$. We approximate the curvature production by $U / r=$ $\omega_{i}$, and $L_{c}$ then becomes

$$
L_{c}=\frac{u_{\tau}}{\kappa \omega_{i}} .
$$

We use $\kappa=0.39$ throughout the chapter, which is consistent with the data of [75], see figure 2.3, and also agrees with measurements of $\kappa$ in turbulent BLs and turbulent channel flows [86]. However, we note that a range of $\kappa$ are reported in literature [64], and the employed data here is not conclusive on the second decimal. A subtle difference with the definition of [14] resides in the definition of the curvature production term. [14] uses the wall normal production only (i.e. $-\frac{2}{r} \overline{u w} U$ ), in strict analogy with the buoyancy production, that contains no streamwise production term. Here, however, we decide to use to sum of the streamwise and wall-normal curvature production terms (i.e. $-\frac{1}{r} \overline{u w} U$ ) to account for the total effects of streamline curvature. Finally, we note that a similar length scale can be derived to account for the effects of spanwise rotation on the flow over a flat wall $[14,87,88]$.

\subsubsection{Development of the functional form of $\omega^{+}\left(y^{+}\right)$}

Figure 2.3(a) shows the angular velocity profiles for turbulent TC flow. For very high $\mathrm{Re}$ of $\mathrm{O}\left(10^{6}\right)$, [75] observed the existence of a logarithmic form of the 

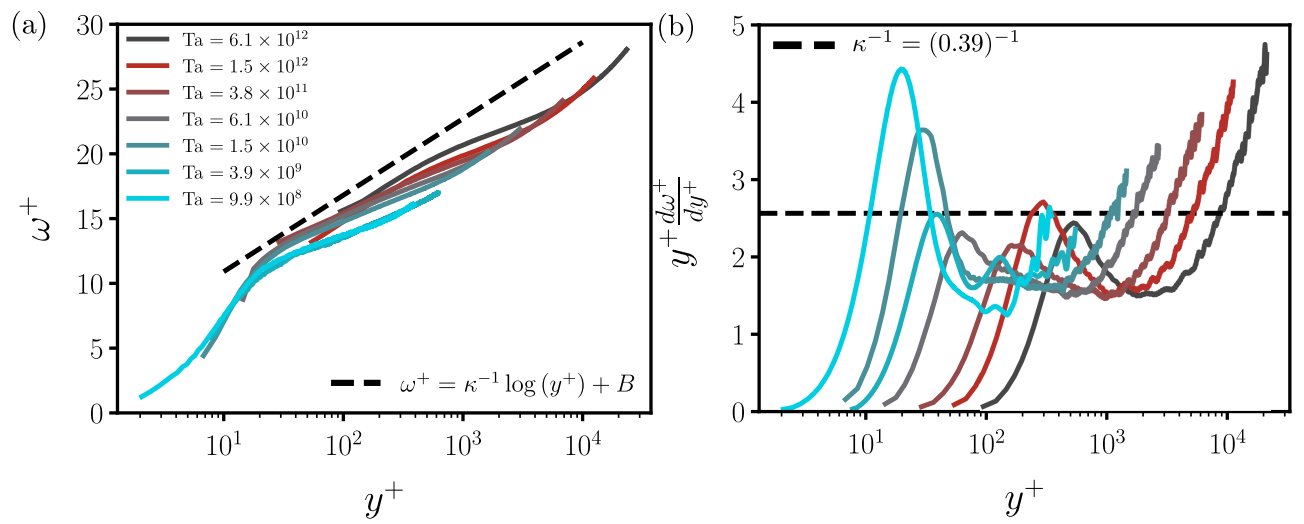

Figure 2.3: The IC BL angular velocity profiles for $\eta=0.716$. (a) Mean angular velocity $\omega^{+}=\left(\omega_{i}-\langle\omega(r)\rangle_{A(r), t}\right) / \omega_{\tau, i}$ versus the wall normal distance $y^{+}=\left(r-r_{i}\right) / \delta_{\nu, i}$. A logarithmic velocity profile with slope $\kappa^{-1}$ is observed in a limited spatial region at the highest Taylor numbers. (b) The diagnostic function reveals a very limited spatial region in which $y^{+} \frac{d \omega^{+}}{d y^{+}}=\kappa^{-1}$, indicated by the dashed line. Data from the PIV measurements of [75].

angular velocity profile with $\kappa \approx 0.39$ and $B \approx 5$, in accordance with (2.12). However, the extent of the profile is very limited, namely $50<y^{+}<600$, covering a much smaller spatial range than it would in canonical wall-turbulence systems as channel flow and flat plate turbulent boundary layers [11] at similar $R e_{\tau}$. Figure 2.3(b) presents the so-called diagnostic function, $y^{+} \frac{d \omega^{+}}{d y^{+}}$, which allows for a more detailed investigation of the log slope of $\omega^{+}\left(y^{+}\right)$. Even for these high Re flows, only a very small region of the profile coincides with the straight line with slope $\kappa^{-1}$, which in this representation represents the log-layer.

Following the analysis in section 2.4.1, we expect the velocity profile to behave differently in the region where curvature effects play a role - in close analogy with the Monin-Obukhov similarity theory. Therefore, we plot the compensated gradient of the velocity profile versus the ratio of turbulence production terms, see equation (2.16), in figure 2.4(a). For clarity we include only the highest three Ta number cases from the dataset of [75]. Indeed, we find that the gradient of the velocity correlates strongly with the relative effects of shear and curvature. Where turbulence production is governed by shear alone, we find that the gradient approximates $\kappa^{-1}$, albeit marginally. However, where curvature effects become significant, i.e. for $10^{0} \leq \frac{r}{U} \frac{d U}{d r} \leq 10^{1}$, we find that 

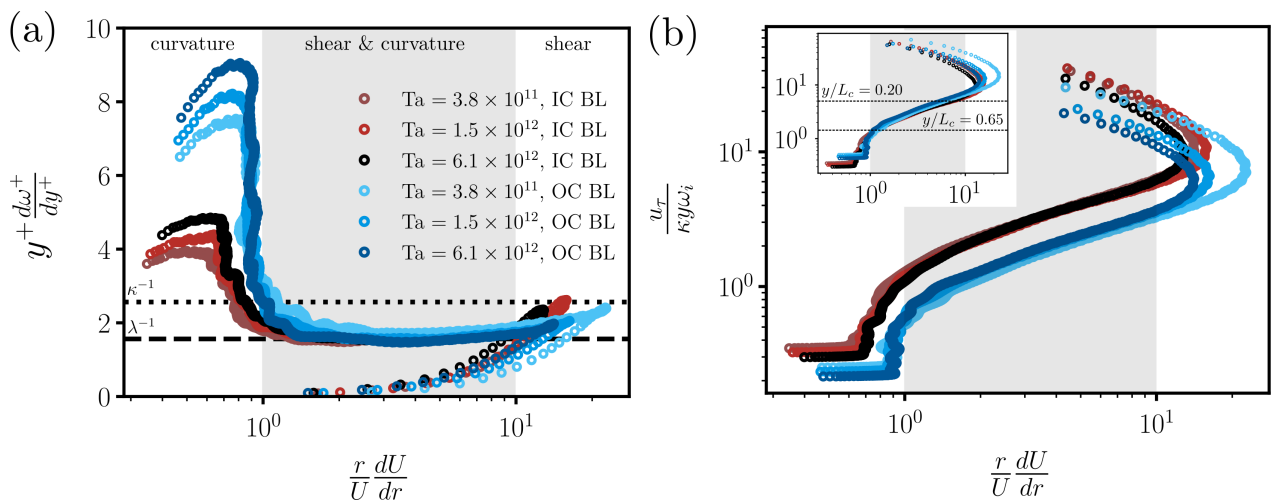

Figure 2.4: (a) Compensated gradient of the mean angular velocity versus the ratio of shear production of turbulence over curvature production of turbulence (see equation (2.16)). (b) The approximation the Obukhov curvature length $L_{c}(y)$ (equation 2.17) versus the exact calculation of the Obukhov curvature length (equation 2.16). Inset of (b) highlights the collapse of IC and OC approximations with the use of different velocity scales (axis labels are the same as figure (b)), respectively $\omega_{i} r_{i}$ for the IC and $0.50 \omega_{i} r_{i}$ for OC. Data from the PIV measurements of [75].

the gradient is $\lambda^{-1}$. It is remarkable that the gradient is constant over such an extended range over which the relative effects of curvature and shear change. For $\frac{r}{U} \frac{d U}{d r} \leq 10^{0}$ curvature effects are dominant and the bulk velocity profile sets in (see section 2.4.3).

Consequently, we make the wall-normal distance dimensionless with $L_{c}$, see (2.17). This is done in figure 2.5(b) where we plot the diagnostic function versus $y / L_{c}$. Similar to figure 2.4 , we find a collapse of the angular velocity profiles, directly justifying the use of $L_{c}$ in turbulent TC flow. The profiles not only collapse with respect to their wall-normal location, but also all plateau at $y^{+} \frac{d \omega^{+}}{d y^{+}}=\lambda^{-1}$, i.e. the slope (in a semi-logarithmic representation) of $\omega^{+}\left(y^{+}\right)$. This secondary flat regime with slope $\lambda^{-1}$ exists for larger $r>L_{c}$, than the $\kappa^{-1}$ regime. We find that $\lambda=0.64$.

From these observations in figure 2.5 we obtain the unknown function $\Phi_{\omega}\left(\frac{y}{L_{c}}\right)$ in $(2.3)$ for $0.20<y / L_{c}<0.65$ :

$$
\Phi_{\omega}\left(\frac{y}{L_{c}}\right)=\frac{1}{\lambda} \approx \frac{1}{0.64} ; \quad 0.20 \lesssim y / L_{c} \lesssim 0.65
$$


Consequently, we integrate $\frac{d \omega^{+}}{d\left(y / L_{c}\right)}=\frac{1}{\left(y / L_{c}\right) \lambda}$ and arrive at

$$
\omega^{+}=\lambda^{-1} \log \left(y / L_{c}\right)+K,
$$

where $K$ is an integration constant and $\log$ is the natural logarithm. The offset $K$ of this second regime at larger $r$ is related to the height at which the first logarithmic regime at smaller $r$ peels off to the second log regime. We thus expect that $K=\kappa^{-1} \log L_{c}^{+}+C$ which results in,

$$
\omega^{+}=\lambda^{-1} \log \left(y^{+}\right)+\left(\kappa^{-1}-\lambda^{-1}\right) \log \left(L_{c}^{+}\right)+C,
$$

where $C$ is a constant equal to 1.0 (obtained by fitting to the highest Taylor number data). If the transition from a shear logarithmic regime (with slope $\kappa^{-1}$ ) to a curvature logarithmic regime (with slope $\lambda^{-1}$ ) occurs exactly at $y^{+}=L_{c}^{+}$, and if this transition is sharp, we would expect to recover the offset of the curvature logarithmic regime as $K=\kappa^{-1} \log L_{c}^{+}+B$. Hence, we would obtain the constant $C=B=5$. However, we see in figure 2.3(a) that the transition between the two logarithmic regions in the flow is not sharp, but gradual. The gradual transition from the shear dominated region to the curvature affected region and the long 'blending' region in between made us decide not to simply equate (4.9) with the von Kármán profile to obtain the lower bound of the curvature logarithmic region (4.7). Instead, as explained, we employ a stricter empirical condition from which we find $y=0.20 L_{c}$. In figure 2.5(a) we plot $\omega^{+}$versus $y / L_{c}$ and subtract $K$ to highlight the collapse. Indeed, we observe a collapse of the profiles in the range $0.20 \lesssim y / L_{c} \lesssim 0.65$.

\subsubsection{The constant angular momentum region in the bulk}

In the previous section we discussed the shape of the mean streamwise velocity profile in the IC BL, culminating in a new functional form which includes the stratification length $L_{c}$. However, to arrive at a $\mathrm{Nu}(\mathrm{Ta})$ relationship, we need to assess the velocity profile in the bulk region, too. [89] already observed that for unstable flows (i.e. IC rotation and a stationary OC) the bulk flow obeys a constant angular momentum $\mathrm{L}=\mathrm{M}_{\mathrm{o}}$. Later, [4] came to a similar conclusion and found that $\mathrm{M}_{\mathrm{o}}=\omega_{i} r_{i}^{2} / 2$ for pure IC rotation. In recent years this finding is often confirmed by new datasets, see e.g. [55], [90], and [15]. This region of constant angular momentum in IC rotating TC flow is reminiscent to a linear mean flow scaling in the bulk of spanwise rotating channel flow $[87,88,91]$. Here, we plot the constant angular momentum region in figure 2.5. We find that the transition from a $\lambda^{-1}$ region into a constant angular momentum 

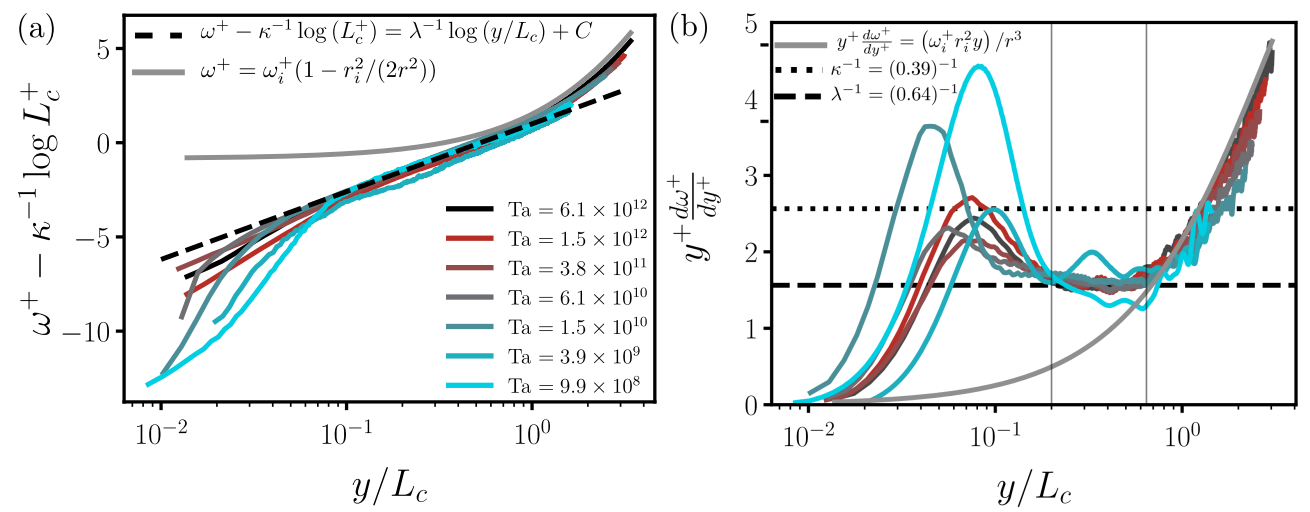

Figure 2.5: The IC BL mean angular velocity profiles for $\eta=0.716$. (a) Mean angular velocity $\omega^{+}=\left(\omega_{i}-\langle\omega(r)\rangle_{A(r), t}\right) / \omega_{\tau, i}$ with the $L_{c}^{+}$dependent offset $\kappa^{-1} \log \left(L_{c}^{+}\right)$subtracted to highlight collapse of the profiles. The curved, thick, grey line is the constant angular momentum $\mathrm{M}_{\mathrm{o}}=\omega_{i} r_{i}^{2} / 2$, as derived by [4], which very closely fits the data at $y>L_{c}$. (b) Diagnostic function versus the rescaled wall normal distance $y / L_{c}=\left(r-r_{i}\right) / L_{c}$, where $L_{c}=u_{\tau, i} /\left(\kappa \omega_{i}\right)$ is the curvature Obukhov length. The vertical grey lines indicate the bounds of the second log region. Data from the PIV measurements of [75].

$\omega^{+}=\omega_{i}^{+}\left(1-r_{i}^{2} /\left(2 r^{2}\right)\right)$ region occurs at $y=L_{c}$. As such, the bulk region is entirely dominated by curvature effects of the streamlines. Consequently, the IC BL thickness $\delta_{i}$ is equal to the curvature Obukhov length, $\delta_{i} \approx L_{c}$ (and OC BL thickness $\delta_{o}=2.5 L_{c}$ ). Recently, a very similar thickness of the BL was empirically found by [15].

\subsubsection{The outer cylinder boundary layer}

Analogous to the IC BL we can analyse the OC BL, with IC rotation only, in the spirit of the Monin-Obukhov similarity theory. As mentioned in section 2.4, [75] also obtained velocity profiles of the OC BL for the highest five Ta number experiments. From (2.16) we derive that the relevant length scale for the $\mathrm{OC} \mathrm{BL}$ is $L_{c, o}=r u_{\tau, o} /(\kappa U)$ with $y=r_{o}-r$. We approximate the velocity $U$ with the scale $\omega_{i} r_{i}$ and the radius of curvature $r$ with $r_{o}$, so that with $u_{\tau, o} r_{o}=u_{\tau, i} r_{i}, L_{c, o}=u_{\tau, i} /\left(\kappa \omega_{i}\right)$. The length scale is the same as $L_{c, i}$. The definition of the Obukhov curvature length $L_{c}=\left(u_{\tau, i} r_{i}\right) /(\kappa U)$ contains a velocity $U$. Although this velocity is a function of the wall normal coordinate, we approximate it by the velocity scale $u_{i}$ of the IC throughout. The difference 

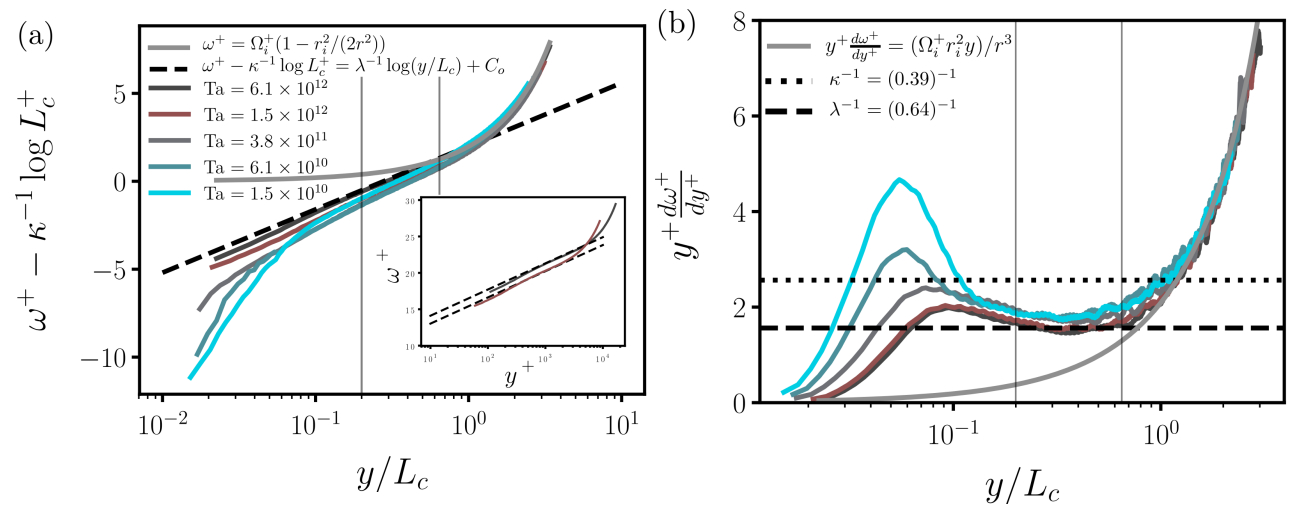

Figure 2.6: The OC BL angular velocity profiles for $\eta=0.716$. (a) Mean angular velocity $\omega^{+}=\langle\omega(r)\rangle_{A(r), t} / \omega_{\tau, o}$ with the $L_{c}^{+}$dependent offset $\kappa^{-1} \log L_{c}^{+}+C_{o}$ subtracted to convey collapse of the profiles. The vertical grey lines indicate the bounds of the second log region. The curved, thick, grey line is the constant angular momentum $\mathrm{M}_{\mathrm{o}}=\omega_{i} r_{i}^{2} / 2$, as derived by [4], which very closely fits the data at $y>L_{c}$. (b) Diagnostic function versus the rescaled wall normal distance $y / L_{c}=\left(r_{o}-r\right) / L_{c}$, where $L_{c}=u_{\tau, i} /\left(\kappa \omega_{i}\right)$ is the curvature Obukhov length. For lower $y\left(y<0.20 L_{c}\right)$ the shear dominated logarithmic regime with slope $\kappa^{-1}$ peels off into a second logarithmic regime with slope $\lambda^{-1}$. The inset to (a) shows the mean angular velocity versus the wall normal distance $y^{+}=\left(r_{o}-r\right) / \delta_{\nu, o}$, where the dashed line is the curvature logarithmic relation. Data from the PIV measurements of [75]. 
between the actual velocity in the IC boundary layer and $u_{i}$ is different from the difference between the actual velocity in the outer cylinder boundary layer and $u_{i}$. In figure 2.4(b) we find that indeed the approximation of $L_{c}$ in equation (2.17) is not consistent for IC and OC when we employ $U=\omega_{i} r_{i}$. However the inset shows that when we use $U=0.50 \omega_{i} r_{i}$ as the velocity scale for the OC (and $U=\omega_{i} r_{i}$ for the IC), the approximation of $L_{c}$ is consistent.

Figure 2.6(b) presents the gradient of the OC BL velocity profiles versus the dimensionless wall-distance $y / L_{c}$. Again, we observe collapse of the profiles in both the vertical direction and the horizontal direction. In the range $0.20<$ $y / L_{c}<0.65$ the gradient of the profiles is $\lambda^{-1}$, whose value is identical to the IC BL profiles. Since the findings in figure 2.6(b) are the same as in figure $2.5(\mathrm{~b})$, we derive the velocity profile for the $\mathrm{OC} \mathrm{BL}$ in the same manner as (2.18-2.20) and arrive at

$$
\omega_{o}^{+}=\lambda^{-1} \log \left(y^{+}\right)+\left(\kappa^{-1}-\lambda^{-1}\right) \log \left(L_{c}^{+}\right)+C_{o},
$$

where $C_{o}=2.0$ is obtained from fits in figure 2.6(a). Again, the profiles in figure 2.6(a) exhibit fair overlap between (2.21) and the experimental data, especially at the highest two Ta numbers (see inset). We note that $R e_{\tau, o}$ at the OC BL is smaller than $R e_{\tau, i}$ at the IC BL, and consequently, we expect that the data at lower Ta still suffers from insufficient scale separation.

\subsection{The effects of the radius ratio $\eta$}

Up to this point, we have shown that one can treat IC rotating TC flow as an unstably stratified turbulent shear flow, in close analogy with temperature stratified flows. We proposed a new functional form of the mean angular velocity in (2.20) that well describes the experimental profiles measured by [75] in both inner and outer BL for all Re at $\eta=0.716$. The question arises what implications of the theory of stratified flows - and consequently (2.20) - bring to TC turbulence at varying radius ratios. To answer this question we first analyse DNS data of [55] and PIV data of [76] at a lower radius ratio of $\eta=0.50$ (corresponding to larger curvature effects), followed by the analysis of the DNS [55] data at a high radius ratio of $\eta=0.909$.

\subsubsection{Radius ratio $\eta=0.5$}

Figure 2.7 presents the velocity profiles at $\eta=0.5$. The black solid line represents DNS data at a remarkable high Ta of $1.1 \times 10^{11}$ resulting in a 

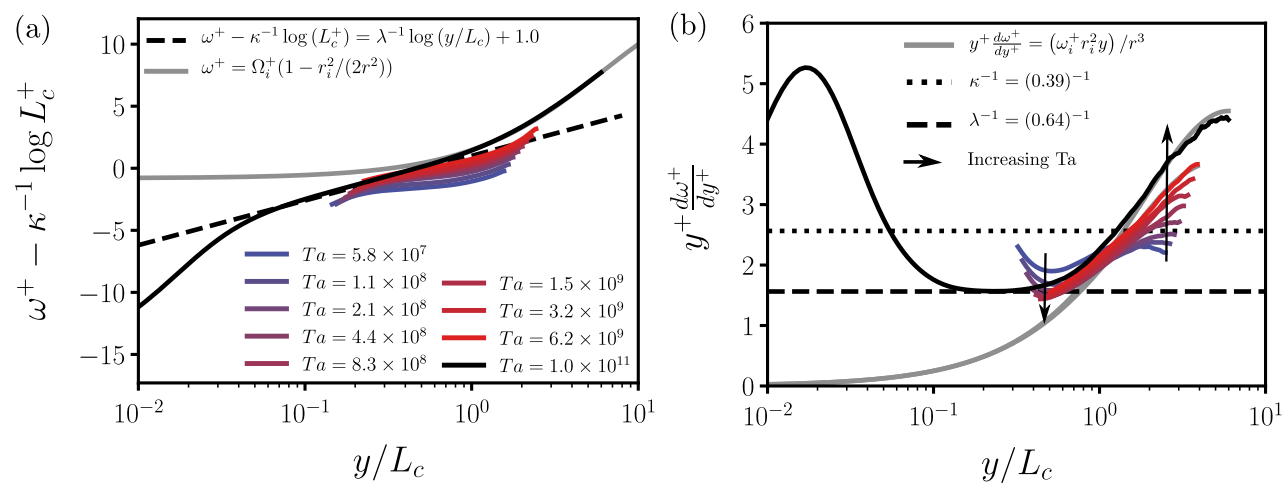

Figure 2.7: The IC BL mean angular velocity profile at $\eta=0.50$. (a) Mean angular velocity $\omega^{+}=\left(\omega_{i}-\langle\omega(r)\rangle_{A(r), t}\right) / \omega_{\tau, i}$ with the $L_{c}^{+}$dependent offset $\kappa^{-1} \log \left(L_{c}^{+}\right)$subtracted to convey collapse of the profiles. The curved, thick, grey line is the constant angular momentum $\mathrm{M}_{\mathrm{o}}=\omega_{i} r_{i}^{2} / 2$, as derived by [4], which very closely fits the data at $y>L_{c}$. The black solid line represents DNS data of [55] whereas the colored lines represent the PIV data by [76]. (b) Diagnostic function versus the rescaled wall normal distance $y / L_{c}=(r-$ $\left.r_{i}\right) / L_{c}$, where $L_{c}=u_{\tau, i} /\left(\kappa \omega_{i}\right)$ is the curvature Obukhov length.

significant scale separation; $R e_{\tau}=3257$, see table 2.1. Nevertheless, the diagnostic function in figure 2.7(b) does not portray a shear dominated $\kappa^{-1}$ regime, i.e. the solid black line never follows the black dotted line. However, between $y / L_{c} \approx 0.20$ and $y / L_{c} \approx 0.65$ the $\lambda^{-1}$ regime is obtained. Note that we do not fit $\lambda^{-1}$ to the data, but only use the value $(\lambda=0.64)$ as obtained in section 2.4. The dark grey solid line departs from the $\lambda^{-1}$ region around $y / L_{c} \approx 0.65$, to follow the $\mathrm{M}_{\mathrm{o}}=\omega_{i} r_{i}^{2} / 2$ scaling of the bulk. This is in agreement with the observations at $\eta=0.716$.

To understand the absence of a $\kappa^{-1}$ region for this low $\eta$, we refer to the scale separation in table 1 . A $\kappa^{-1}$ slope requires that $30<y^{+} \ll 0.20 L_{c}^{+}$. However, for $\eta=0.50$ at $\mathrm{Ta}=1.1 \times 10^{11}$ we find that $0.20 L_{c}^{+}=109$. This marginal scale separation is insufficient to find a logarithmic velocity profile with slope $\kappa^{-1}$. However, the scale separation seems to be sufficient to determine the offset of the curvature logarithmic part of the velocity profile, i.e. $K=1 / \kappa \log \left(L_{c}^{+}\right)+$ 1.0 in equation (2.19) shown in figure 2.7(a). A large separation of scales between $L_{c}^{+}$and $R e_{\tau}$ results in a large curvature dominated flow region where the angular momentum becomes constant, see figure 2.7(a).

Figure 2.7 also presents PIV data for low Ta from [76]. Although the scale 

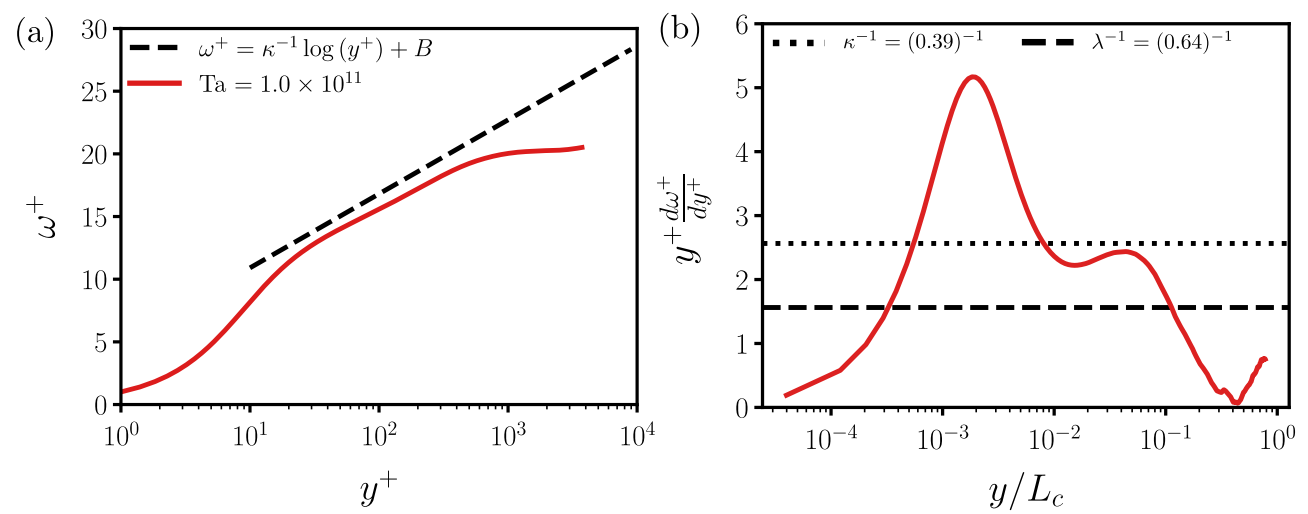

Figure 2.8: (a) Mean angular velocity $\omega^{+}=\left(\omega_{i}-\langle\omega(r)\rangle_{A(r), t}\right) / \omega_{\tau, i}$ versus the wall normal distance $y^{+}$. The red solid line is DNS data taken from [55]. (b) Diagnostic function versus the rescaled wall normal distance $y / L_{c}=(r-$ $\left.r_{i}\right) / L_{c}$, where $L_{c}=u_{\tau, i} /\left(\kappa \omega_{i}\right)$ is the curvature Obukhov length, for $\eta=0.909$.

separation is limited for $R e_{\tau}<1000$ we find a that with increasing Ta the profiles convergence to the $\lambda^{-1}$ region. For the very low Taylor number cases, the offset is too low to reach the dashed line, indicating that the shear logarithmic region is absent. This is confirmed by the absence of sufficient scale separation (i.e. $0.2 L_{c}^{+}<30$, with $y^{+}=30$ the conventional start of the shear logarithmic region [11]) to form a shear logarithmic regime, see table 1 in the appendix. However, for $0.2 L_{c}^{+}>30$ (at $T a \geq 3.2 \times 10^{9}$ ), the offset of the curvature region is correctly set by the shear velocity logarithmic profile. Hence, the profiles follow the prediction.

\subsubsection{Radius ratio $\eta=0.909$}

Figure 2.8 shows data from a DNS at high $\eta=0.909$ (corresponding to small curvature effects) and $\mathrm{Ta}=1.0 \times 10^{11}$. Interestingly, we observe a pronounced $\kappa^{-1}$ region. However there is a total absence of the $\lambda^{-1}$ and the $\mathrm{M}_{\mathrm{o}}$ region. Once again this is understood with the scale separation argument. In this case $L_{c}^{+}>R e_{\tau}$, and therefore there is no location in the flow where the curvature effects are significant, see table 2.1. 


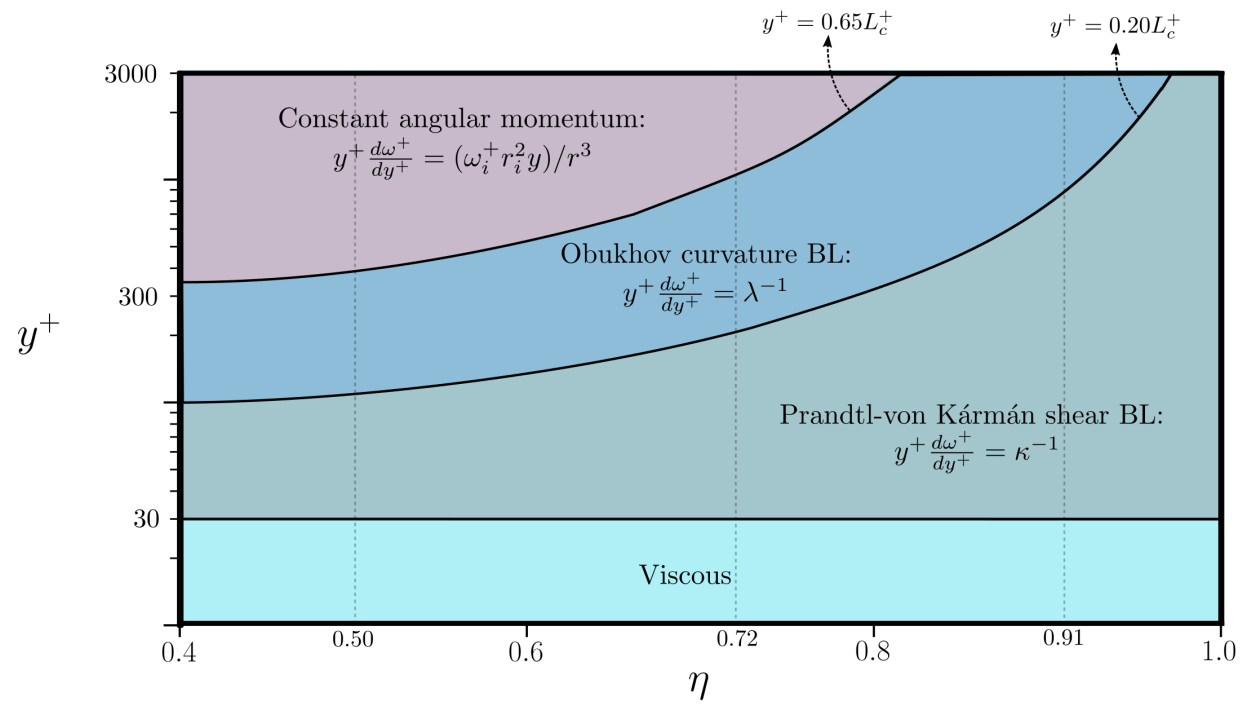

Figure 2.9: Varying regimes in between the solid boundary (here the IC wall at $y^{+}=0$ ) and the outer length scale at $y^{+}=R e_{\tau}$ for increasing radius ratio $\eta$, from $\eta=0.4$ (strong curvature) to $\eta=1.0$ (no curvature). The diagram is based on the values of $L_{c}^{+}$at $R e_{\tau} \approx 3000$ for $\eta=(0.500,0.716,0.909)$, see table 2.1 in the appendix. 


\subsubsection{General radius ratio $\eta$}

To close this section, we provide a phase diagram of the scale separation at $R e_{\tau} \approx 3000$ for varying $\eta$, in order to illustrate where one would expect to see $\kappa^{-1}, \lambda^{-1}$, and constant angular momentum regions of the angular velocity profile, in figure 2.9. We base the phase diagram on three cases for $\eta=$ $(0.500,0.716,0.909)$ and $R e_{\tau} \approx 3000$, for which we have the phase boundaries, see table 2.1. Note that the boundaries are not sharp, and gradual changes in the relative importance of TKE production by shear and curvature lead to new regions. However, we now immediately see from the diagram that for high $\eta$ the Obukhov curvature BL is only expected to appear distinctly at extremely high $R e_{\tau}$ (higher than $R e_{\tau}=3000$ ). In contrast, for low $\eta$, we need extremely high $R e_{\tau}$ (higher than $R e_{\tau}=3000$ ) to observe the Prandtl-von Kármán turbulent BL type.

\subsection{The $\mathrm{Nu}(\mathrm{Ta})$ and $\mathrm{C}_{\mathrm{f}}\left(\mathrm{Re}_{\mathrm{i}}\right)$ relationships}

The derivation of the angular velocity profile in a turbulent BL with strong curvature effects, see (2.20), allows us to obtain a functional form that relates the dimensionless torque $\mathrm{Nu}$ to the dimensionless driving $\mathrm{Ta}$ at $R e_{o}=0$. To do so, we follow the very recent work by [15]. Therein, the BL profile (the conventional shear dominated von Kármán type) is matched with the constant angular momentum bulk profile at the edge of the BL. With a fitting constant for the BL thickness, [15] arrive at a very accurate calculation of $\mathrm{Nu}$ over a wide range of Ta. Here, we match the angular velocity profiles in the bulk and the $\mathrm{BL}$ at the BL height $\delta=\alpha L_{c}$. Note that the constant $\alpha$ is easily extracted from figure 2.5, where it refers to the outer bound of the $\lambda^{-1}$ region - where the BL and bulk meet.

$$
\frac{\omega_{i}}{\omega_{\tau, i}}-\frac{1}{\lambda} \log \alpha L_{c}^{+}-\left(\frac{1}{\kappa}-\frac{1}{\lambda}\right) \log L_{c}^{+}-C=\frac{\omega_{i} r_{i}^{2}}{2 \omega_{\tau, i}\left(r_{i}+\alpha L_{c}\right)^{2}} .
$$

Note that equation (6.1) is equivalent of [15] (4.5-4.7) with differences of $O\left(R e_{\tau} / R e_{i}\right)$, and with a different constant. Upon closely following [15] including $R e_{\tau}<<R e_{i}$, equation (6.3) results,

$\mathrm{Nu}=\frac{\kappa^{2} \eta^{3} \mathrm{Ta}^{1 / 2}}{4(1+\eta)^{2} W(Z)^{2}}, \quad Z=\sqrt{\frac{\kappa \eta^{3} \mathrm{Ta}^{1 / 2}}{2(1-\eta)(1+\eta)^{3}}} \exp \left(\frac{\kappa\left(C+\frac{1}{\lambda} \log \alpha\right)}{2}\right)$, 


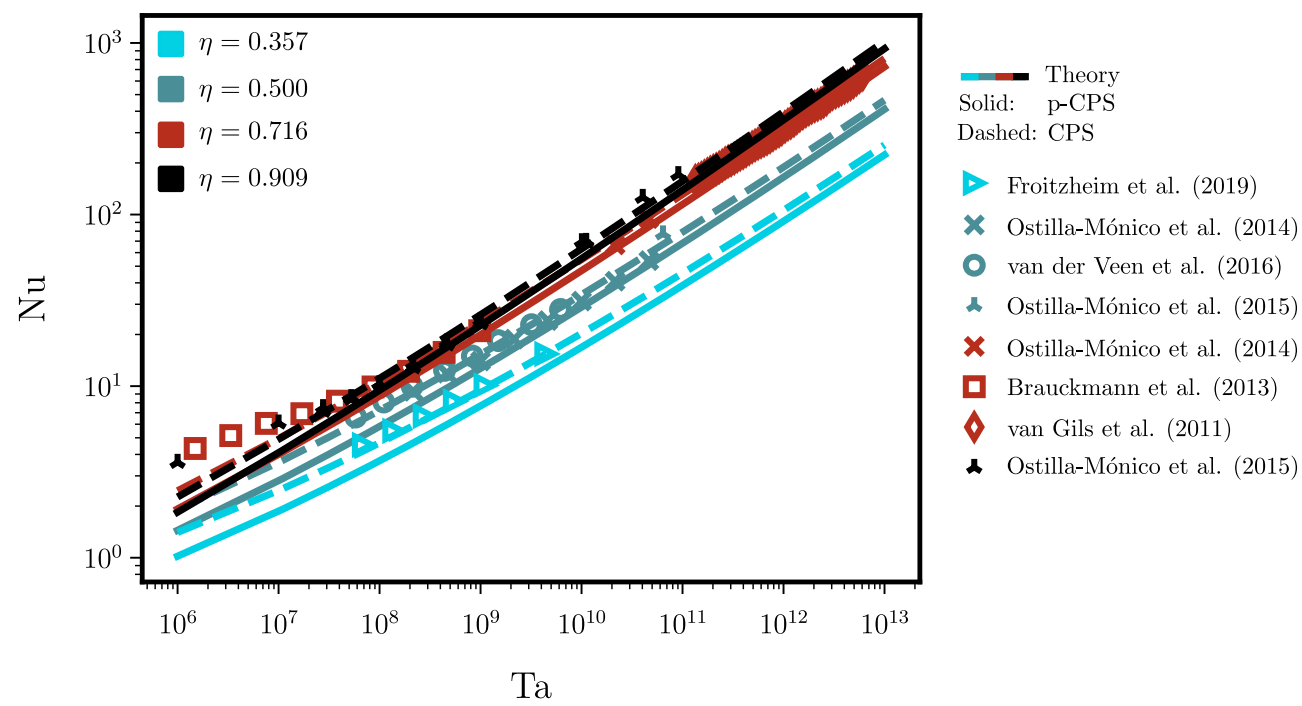

Figure 2.10: The dimensionless torque $\mathrm{Nu}$ versus the dimensionless rotation rate Ta of the IC. Solid lines represent the result as obtained by the matching of profiles in section 6 , with the resulting relationship $\mathrm{Nu}(\mathrm{Ta})$ given by $(2.23)$ (present p-CPS). Dashed lines represent the result of [15] (CPS). Symbols are the values of $\mathrm{Nu}$ obtained by DNS or experiments; $\eta=0.357$ (blue triangle) [92], $\eta=0.500$ (crosses) [93], (open circles) [76] and (triangle) [55], $\eta=0.716$ (squares) [47], (crosses) [93] and (diamonds) [94], $\eta=0.909$ (triangles) [55]. 

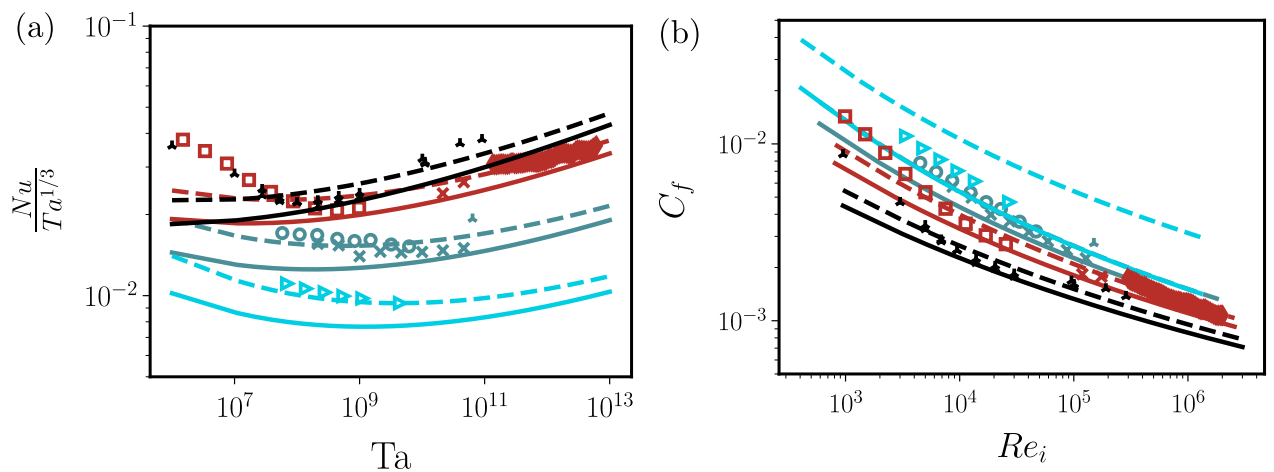

Figure 2.11: (a) The dimensionless torque $\mathrm{Nu}$, compensated with the scaling of TC flow with laminar BL and turbulent bulk $\mathrm{Ta}^{1 / 3}$, versus the dimensionless rotation rate Ta of the IC. (b) The friction factor $C_{f}$ versus the the IC Reynolds number $\operatorname{Re}_{i}$. Colours and symbols are the same as in figure 2.10 and links to the references can be found in the caption of that figure.

where $W(Z)$ is the principal branch of the Lambert $W$ function. We note that equation (2.23) is different from the result of [15] (4.15-4.16) by only a constant in the argument of the productlog function and the difference in $\mathrm{Nu}(\mathrm{Ta})$ is only minor. We refer to equation (6.2) as the 'present CPS model' (i.e. p-CPS).

Figure 2.10 presents equation (2.23) together with 8 datasets from DNS and experiments - covering $0.357 \leq \eta \leq 0.909$ and 7 orders of magnitude in Ta. $\alpha=0.65$, see (2.18) and figure 2.5. Naturally, we find deviations at low Ta, where the BLs are not fully turbulent yet. However, we find good overlap at high Ta for various $\eta$. For high $\eta$ (at $R e_{\tau}=3000, \eta \gtrsim 0.80$ ), (2.23) loses its validity since shear is dominating curvature effects throughout the entire $\mathrm{BL}$ at the current $\mathrm{Ta}$. The $\mathrm{Nu}(\mathrm{Ta})$ relation is thus better described by the functional form obtained in [15]. However, we note that the ratio $R e_{\tau} / L_{c}^{+}$will become larger with increasing Ta, so that for extremely high Ta (even much higher than $10^{12}$ ), the $\mathrm{Nu}(\mathrm{Ta})$ relationship at $\eta=0.909$ will also follow (2.23). For $\mathrm{Ta}<10^{6}$, the BLs are of the laminar type and $\mathrm{Nu}$ scales with $\mathrm{Ta}^{1 / 3}$ [45]. Figure 2.11(a) shows the $\mathrm{Nu}(\mathrm{Ta})$ relationship where $\mathrm{Nu}$ is compensated with $\mathrm{Ta}^{1 / 3}$, such that we highlight the transition to a turbulent BL where the scaling exponent is larger than $1 / 3$. We emphasize that only after this transition, which is gradual and appears to depend on $\eta$, when BLs are entirely turbulent, equation (2.23) will correctly calculate $\mathrm{Nu}(\mathrm{Ta})$. Figure 2.11(b) presents the 
$\mathrm{C}_{f}\left(\mathrm{Re}_{i}\right)$ diagram, which is more conventionally used in the pipe flow and BL flow communities. The solid lines are given by equation (2.23) where the friction factor is calculated from $C_{f}=4 \mathrm{Nu} /\left(\eta(1+\eta) \operatorname{Re}_{i}\right)$.

\subsection{Summary and conclusions}

In summary, we have developed a theory, similar to that of thermally stratified turbulent BLs, as famously developed by [66], for the curved turbulent BLs in inner cylinder rotating TC flow. In this analogy, the destabilizing effects from curvature of the streamlines in inner cylinder rotating TC flow are similar to the destabilizing effects coming from unstable thermal stratification in the atmospheric BL.

We show that the curvature Obukhov length $L_{c}$ [14] separates the spatial regions that are dominated by shear and curvature effects. We find that for $\delta_{\nu}<y \lesssim 0.20 L_{c}$, the mean angular velocity profile in the BL is described by the classical shear profile, with the slope given by the von Kármán constant $\kappa^{-1}=0.39^{-1}$. In contrast, for $0.20 L_{c} \lesssim y \lesssim 0.65 L_{c}$, where curvature effects are relevant, the slope of the angular velocity profile is $\lambda^{-1}=0.64^{-1}$. For $y \gtrsim 0.65 L_{c}$ curvature effects dominate, and a region with constant angular momentum sets in. This theory is applied to - and found consistent with PIV measurements and high-fidelity DNS data covering a wide range of radius ratios $0.50 \leq \eta \leq 0.909$ and rotation rates $10^{8} \leq \mathrm{Ta} \leq 10^{12}$, and describes both the IC BL and the OC BL.

Building on these findings we obtain a new functional form of the mean angular velocity profile in TC turbulence, with separate spatial regions where curvature and shear effects are respectively relevant. In implementing the [15] theory by matching our new outer boundary-layer profile with the constant angular momentum profile in the bulk at the edge of the BL, we recover their $N u(T a, \eta)$ (and $C_{f}\left(R e_{i}, \eta\right)$ ) relations but with a different constant. For the present smooth-wall flow with the outer cylinder stationary, this supports their model, with fair agreement with various data sets at high $T a$ and different $\eta$. The key assumptions made by [14], namely that production of turbulent kinetic energy is locally balanced by dissipation, as also employed in this research, are left to be addressed by means of DNS at very high Reynolds numbers of $R e=O\left(10^{6}\right)$. Whether the Obukhov (outer) logarithmic region will survive at arbitrary high $R e_{i}$ remains an open question. Open questions also concern the effects of stably stratified TC flow (i.e. outer cylinder rotation), or even mixed stratified TC flow (i.e. counter cylinder rotation) within the framework 
of the Monin-Obukhov similarity theory. However, so far, only velocity profiles with a scale separation up to $R e_{\tau} \approx 1200$ are available for OC rotation [95] to apply the theoretical analysis. Also, based on the newly derived velocity profile, it becomes necessary to reassess the fully rough asymptote for rough wall turbulent TC flow [96]. 

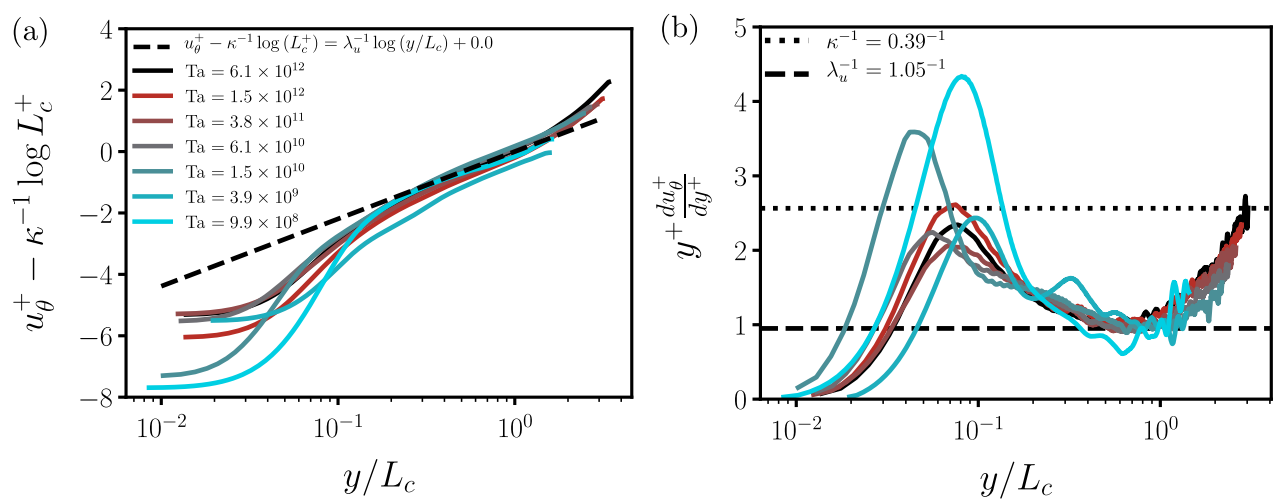

Figure 2.12: The inner cylinder BL mean azimuthal velocity profiles for $\eta=$ 0.716. (a) Mean azimuthal velocity $u_{\theta}^{+}=\left(u_{\theta, i}-\left\langle u_{\theta}(r)\right\rangle_{A(r), t}\right) / u_{\tau, i}$ with the $L_{c}^{+}$ dependent offset $\kappa^{-1} \log \left(L_{c}^{+}\right)$subtracted to highlight collapse of the profiles. (b) Diagnostic function versus the rescaled wall normal distance $y / L_{c}=(r-$ $\left.r_{i}\right) / L_{c}$, where $L_{c}=u_{\tau, i} /\left(\kappa \omega_{i}\right)$ is the curvature Obukhov length. Note that $\lambda_{u}^{-1}$ is different than $\lambda^{-1}$ in the main text. Data from the PIV measurements of $[75]$.

\section{Appendix}

See table 1 for an overview of the used datasets. 


\begin{tabular}{cccccc}
$\eta$ & $\mathrm{Ta}$ & $R e_{i}$ & $L_{c}^{+}$ & $R e_{\tau}$ & \\
\hline & & {$[75]$} & & & \\
0.716 & $9.9 \times 10^{8}$ & $2.6 \times 10^{4}$ & 242 & 488 & PIV \\
0.716 & $3.8 \times 10^{9}$ & $5.0 \times 10^{4}$ & 395 & 877 & PIV \\
0.716 & $1.5 \times 10^{10}$ & $1.0 \times 10^{5}$ & 661 & 1602 & PIV \\
0.716 & $6.1 \times 10^{10}$ & $2.0 \times 10^{5}$ & 1124 & 2950 & PIV \\
0.716 & $3.8 \times 10^{11}$ & $5.0 \times 10^{5}$ & 2327 & 6716 & PIV \\
0.716 & $1.5 \times 10^{12}$ & $1.0 \times 10^{6}$ & 3947 & 12217 & PIV \\
0.716 & $6.1 \times 10^{12}$ & $2.0 \times 10^{6}$ & 6870 & 23093 & PIV \\
\hline & & {$[76]$} & & & \\
0.500 & $5.8 \times 10^{7}$ & $4.5 \times 10^{3}$ & 45 & 141 & PIV \\
0.500 & $1.1 \times 10^{8}$ & $6.2 \times 10^{3}$ & 55 & 183 & PIV \\
0.500 & $2.1 \times 10^{8}$ & $8.6 \times 10^{3}$ & 67 & 239 & PIV \\
0.500 & $4.4 \times 10^{8}$ & $1.2 \times 10^{4}$ & 84 & 320 & PIV \\
0.500 & $8.3 \times 10^{8}$ & $1.7 \times 10^{4}$ & 103 & 413 & PIV \\
0.500 & $1.5 \times 10^{9}$ & $2.3 \times 10^{4}$ & 125 & 531 & PIV \\
0.500 & $3.2 \times 10^{9}$ & $3.4 \times 10^{4}$ & 156 & 714 & PIV \\
0.500 & $6.2 \times 10^{9}$ & $4.7 \times 10^{4}$ & 192 & 933 & PIV \\
\hline & & {$[55]$} & & & \\
0.500 & $1.1 \times 10^{11}$ & $2.0 \times 10^{5}$ & 544 & 3257 & DNS \\
0.909 & $1.0 \times 10^{11}$ & $3.0 \times 10^{5}$ & 4794 & 3745 & DNS
\end{tabular}

Table 2.1: Used datasets. The curvature Obukhov length $L_{c}^{+}$and friction Reynolds number $R e_{\tau}$ at varying $\mathrm{Ta}, R e_{i}$ and radius ratio $\eta$. 


\section{Chapter 3}

\section{Characterizing the drag properties of rough surfaces with a Taylor-Couette setup}

Mapping any given roughness geometry to its fluid dynamic behaviour has been hampered by the lack of accurate and direct measurements of skin-friction drag. Here the Taylor-Couette (TC) system provides an opportunity as it is a closed system and allows to directly and reliably measure the skin-friction. However, the wall-curvature potentially complicates the connection between the wall friction and the wall roughness characteristics. Here we investigate the effects of a hydrodynamically fully rough surface on highly turbulent, inner cylinder rotating, TC flow. We find that the effects of a hydrodynamically fully rough surface on TC turbulence, where the roughness height $k$ is three orders of magnitude smaller than the Obukhov curvature length $L_{c}$ [97], are similar to those effects of a fully rough surface on a flat plate turbulent boundary layer (BL). Hence, the value of the equivalent sand grain height $k_{s}$, that characterizes the drag properties of a rough surface, is similar to those found for comparable sandpaper surfaces in a flat plate BL. Next, we obtain the dependence of the torque (skin-friction drag) on the Reynolds number for given wall roughness, and find agreement with the experimental results within $5 \%$. Our findings demonstrate that global torque measurements in the TC facility are well suited to reliably deduce wall drag properties for any rough surface.

\footnotetext{
Submitted as: P. Berghout, P. A. Bullee, T. Fuchs, S. Scharnowski, C. J. Kähler, D. Chung, D. Lohse and S. G. Huisman, Characterizing the drag properties of rough surfaces with a Taylor-Couette setup, Submitted to J. Fluid Mech.

P. Berghout and P. A. Bullee are shared first author.
} 


\subsection{Introduction}

\subsubsection{Turbulent boundary layers over fully rough walls}

The transport of a fluid over a solid body or the transport of a solid body through a fluid is always hindered by friction forces acting on the interface between the solid and the fluid. Ideally, the solid surface is smooth, and the drag force is a purely viscous force. In nature and engineering applications, however, solid surfaces are nearly always rough. This means that in addition to a modified viscous force, the roughness also results in a pressure contribution to the drag force ('pressure drag'), and consequently, an increase in the total drag force (the so-called 'drag penalty'). For most roughness types, the contribution of the pressure drag to the total friction drag at the surface grows with increasing roughness height. Ultimately, when the pressure drag dominates, the surface is called hydrodynamically fully rough.

Due to the obvious interest in reducing the drag penalty, substantial research has been carried out to investigate the effects of rough surfaces on wall-bounded turbulent flows $[5,6,23]$. The key effect thereof is a downward shift (by $\Delta u^{+}$) of the mean streamwise velocity $\left(u^{+}\right)$in the overlap (or logarithmic) region of the turbulent $\mathrm{BL}[8,9]$. This shift can be considered as a direct measure of the drag penalty. The mean velocity profile for a rough wall in the overlap region is given by the Prandtl-von Kármán profile for smooth walls, minus this shift [11]

$$
u^{+}=\frac{1}{\kappa} \log y^{+}+A-\Delta u^{+},
$$

where $y^{+}$is the wall-normal distance and the von Kármán constant $\kappa \approx 0.40$ and $A \approx 5.0$ are extracted from experimental or numerical data. The superscript ' + ' as usual indicates a normalization with the viscous velocity scale $u_{\tau}=\sqrt{\tau_{w} / \rho}$ and the viscous length scale $\delta_{\nu}=\nu / u_{\tau}$, where $\tau_{w}$ is the wall shear stress, $\rho$ the fluid density and $\nu$ is the kinematic viscosity. For a fully rough surface, it can be derived from dimensional arguments that the velocity shift $\Delta u^{+}$depends logarithmically on the roughness height $k^{+}$, see e.g. $[7,11]$. The so-called fully rough asymptote of the roughness function is given by

$$
\Delta u^{+}=\frac{1}{\kappa} \log k_{s}^{+}+A-B,
$$

where $B \approx 8.5$ is the Nikuradse constant. The equivalent sand grain roughness height $k_{s}^{+}$is obtained by fitting, such that the velocity shift of any fully rough 
surface collapses with the velocity shift of sand grains in turbulent pipe flow, that historically grew to be the reference case [10]. Hence, the key objective in research of wall bounded turbulent flows over rough surfaces is to relate the statistics of a rough surface to the value of $k_{s}$, which characterizes the roughness [98].

\subsubsection{Taylor-Couette flow}

TC flow - the flow between two coaxial, independently, rotating cylinders - is a canonical system in turbulence $[13,99]$. Since the domain is closed in all directions, global balances can be derived and monitored, giving room for extensive comparison between theory, experiments and simulations. Moreover, the torque (skin-friction) can be measured accurately and directly [100,101], in contrast to measurements of skin-friction in open systems.

The forcing strength of the system is quantified by the ratio of the centrifugal force and the viscous force, i.e. the Taylor number

$$
T a=\frac{1}{4}\left(\frac{1+\eta}{2 \sqrt{\eta}}\right)^{4} \frac{\left(r_{o}-r_{i}\right)^{2}\left(r_{i}+r_{o}\right)^{2}\left(\omega_{i}-\omega_{o}\right)^{2}}{\nu^{2}} .
$$

Here $\eta$ is the geometric measure of curvature, namely the ratio $r_{i} / r_{o}$ of the radii of the cylinders. The subscripts $i$ and $o$ indicate inner cylinder and outer cylinder, respectively. The angular velocity is denoted by $\omega$, and $\nu$ is the kinematic viscosity.

The global response of the system is expressed as the Nusselt number $N u_{\omega}$, which is the ratio between the angular velocity flux $J^{\omega}$ in radial direction and its laminar counterpart $J_{\text {lam }}^{\omega}[48]$, as

$$
N u_{\omega}=\frac{J^{\omega}}{J_{\text {lam }}^{\omega}}=\frac{r^{3}\left(\left\langle u_{r} \omega\right\rangle_{A(r), t}-\nu \partial_{r}\langle\omega\rangle_{A(r), t}\right)}{2 \nu r_{i}^{2} r_{o}^{2}\left(\omega_{i}-\omega_{o}\right) /\left(r_{o}^{2}-r_{i}^{2}\right)} .
$$

Here, $\langle\cdot\rangle_{A(r), t}$ denotes averaging over the cylinder surface $A(r)$ and over time $t$. The Nusselt number $N u_{\omega}$ is related to the torque $\mathcal{T}$ required to drive the inner cylinder. In non-dimensional form the torque can be expressed as

$$
G=\frac{\mathcal{T}}{2 \pi L \rho \nu^{2}}=N u_{\omega} \frac{J_{\text {lam }}^{\omega}}{\nu^{2}} .
$$

From here onwards, we assume inner cylinder rotation only, hence $\omega_{o}=0$, as this corresponds to our experiments where we kept the (smooth) outer cylinder stationary at all times. 
The torque is directly related to the wall shear stress $\tau_{w}=\mathcal{T} /\left(2 \pi r_{i}^{2} L\right)$. As commonly used in other canonical systems (e.g. the flat plate BL), we define the friction factor $C_{f}$ as [49]:

$$
C_{f}=\frac{2 \pi \tau_{w, i}}{\rho d^{2} \omega_{i}^{2}}=2 \pi N u_{\omega} J_{l a m}^{\omega}\left(\nu R e_{i}\right)^{-2},
$$

where $R e_{i}=r_{i} \omega_{i} d / \nu$ and $d=r_{o}-r_{i}$. This relation allows for straightforward comparison with other canonical wall-bounded flows like pipe flow, channel flow, flow over a flat plate, etc.

The turbulent flow in the TC setup is strongly influenced by the curvature of its bounding walls, i.e. the cylinders that drive the flow. This distinguishes turbulent TC flow from turbulent flows in other canonical systems. [14] realized that the effects of curvature on a turbulent BL are very similar to the effects of buoyancy stratification on a turbulent BL [65]. In analogy to the Obukhov length $[1,65]$, he derived a length scale that separates the curved BL in a region where the effects of shear dominate (i.e. production of turbulence is dominated by shear production), and a region further away from the wall where curvature effects dominate (i.e. the production of turbulence is dominated by curvature). For smooth wall TC turbulence, this 'curvature Obukhov length' is well approximated by [97]

$$
L_{c, s}=\frac{u_{\tau}}{\kappa \omega_{i}},
$$

where shear dominates for $0.20 L_{c, s} \lesssim y$, shear and curvature effects are both significant for $0.20 L_{c, s} \lesssim y \lesssim 0.65$, and curvature effects dominate at $0.65 L_{c, s} \lesssim$ $y$. Using data of the mean velocity profiles from PIV in turbulent TC flow $[27$, 76] and direct numerical simulations (DNS) [55], the mean angular logarithmic velocity profile in the region of the turbulent $\mathrm{BL}$ where curvature effects are important was recently obtained for smooth wall TC flow [97]. By employing a matching argument between the velocity profiles of the turbulent BL and the bulk region, following the work of [15], an analytical expression for $N u(T a)$ was derived [97].

The effects of irregular boundaries (extended transverse bars in the 'obstacle regime', as referred to by [6]) on turbulent TC flow was previously investigated by means of experiments $[39,40,43,102]$ and DNS $[42,43]$. Here the ratio $k / d$ between the height of the bars and the gap width $d=r_{o}-r_{i}$ was as large as $k / d=0.05$ or even 0.1. Later [96] numerically studied the effects of sand grain roughness $(k / d=0.019-0.087)$ on the turbulent TC velocity profiles, and found similar transitionally rough behaviour as the sand grain roughness 
of [10] in turbulent pipe flow. However, we note that both the experimental and computational studies in TC flow suffered from limited scale separation between the roughness scale $k$ and the gap width $d$.

In this chapter, we study the effects of a hydrodynamically fully rough inner cylinder on the turbulent wall-bounded flow, with small roughness $k / d=$ 0.014 , where $k \equiv 6 k_{\sigma}$, and $k_{\sigma}$ is the standard deviation of the roughness elevation. In particular, we keep $k$ smaller than the curvature Obukhov length $L_{c}$ (see (3.11)), namely $k / L_{c}=0.078-0.090$. We will demonstrate that in order to study the effects of roughness on a turbulent flow in TC, $k \ll d$ is not enough. Rather, $k \ll L_{c}$ must also hold, to ensure that effects related to the streamwise curved geometry are not influencing the effects of the roughness.

Hence, we hypothesize that effects of roughness in TC turbulence (where $\left.k \ll L_{c}\right)$ are similar to the effects of roughness in canonical systems without streamwise curvature. Thus global measurements in the TC facility can be employed to characterize drag properties of the rough surface. The outer cylinder remains smooth, to allow for optical access of the velocity profiles.

The chapter is organised as follows: In section 3.2, we describe the experimental methods. We then (section 3.3) discuss the relevant dynamical length scales in the experiment, and elaborate on the different regions in the BL where turbulent production is dominated by shear effects, and where effects related to the streamwise curvature of the setup play a role. We also comment on the scale separation and show that the roughness mainly affects the inertial shear dominated regime, and hence, effects from the streamwise curved geometry of the TC flow do not modify the velocity shift. In section 3.4 we use the mean velocity profiles of the inner cylinder boundary layer, to show that apart from the shift, the velocity profiles for rough and smooth inner cylinders are the same. We use this in section 3.5 to calculate the angular velocity shift $\Delta \omega^{+}$, from which the equivalent sand grain roughness height is determined in section 3.6. In section 3.7 we demonstrate that the bulk region of the flow is of constant angular momentum, which is used in section 3.8 to obtain a relation between the torque (skin-friction drag) and the Reynolds number for given surface roughness $k_{s}$, in agreement with our experimental results. The chapter ends with a summary, conclusions, and an outlook (section 3.9). 


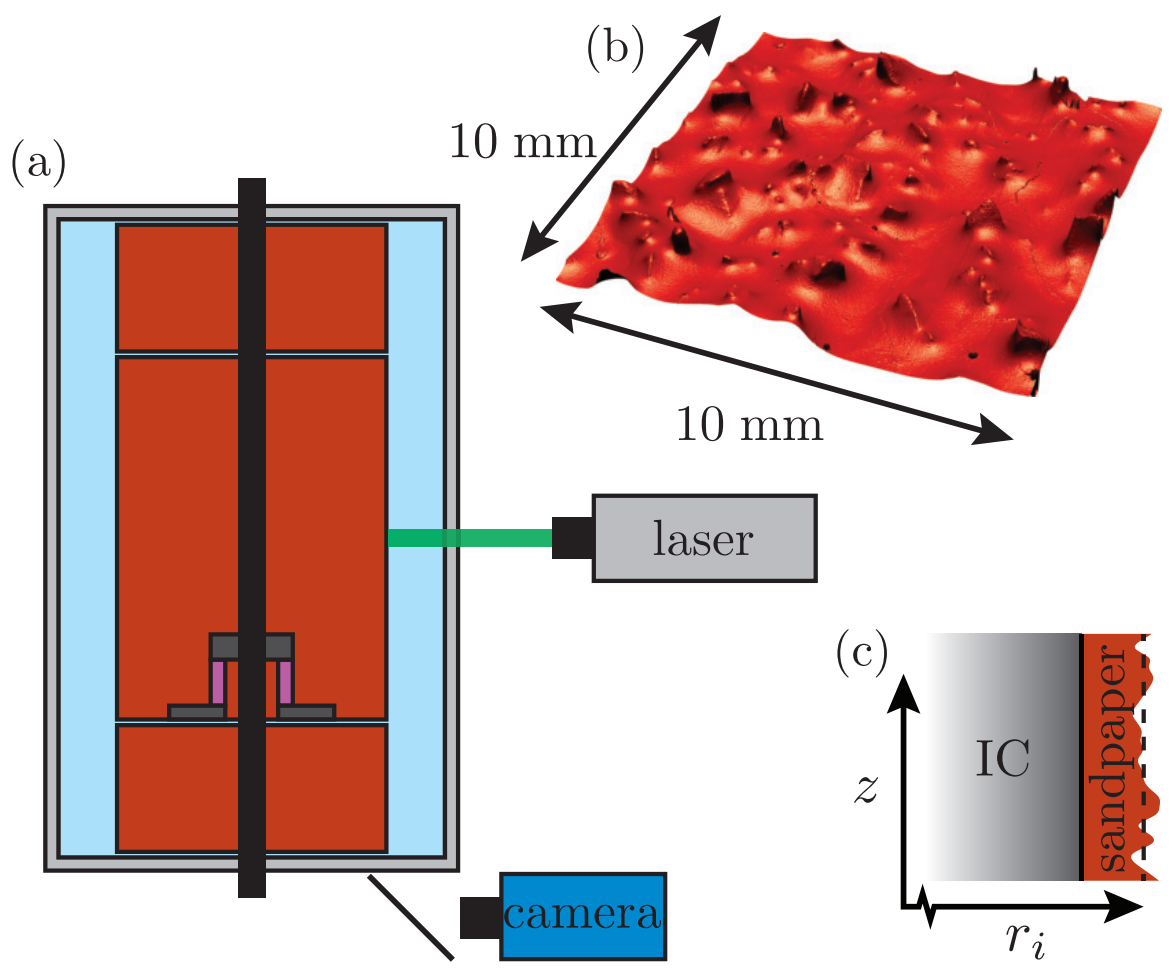

Figure 3.1: (a) Cross section of the TC geometry. The tracer particles are illuminated by a $1 \mathrm{~mm}$ thick horizontal laser light-sheet from the right. The light scattered by the tracers is imaged from the bottom through a mirror. The torque sensor only measured the torque of the middle part of the inner cylinder. (b) 3D visualization of the confocal scan of the used sandpaper. (c) Cross-section of the inner cylinder with the sandpaper attached to the surface. 


\subsection{Experimental setup and methods}

\subsubsection{Experimental setup}

The experiments were performed in the Twente Turbulent Taylor-Couette $\left(\mathrm{T}^{3} \mathrm{C}\right)$ facility [80], with water as working liquid. We used a fully rough inner cylinder with an outer radius of $r_{i}=201.2 \mathrm{~mm}$, and a transparent outer cylinder with an inner radius of $r_{o}=279.4 \mathrm{~mm}$. This gives a radius ratio of $\eta=0.720$ and a gap width $d=78.2 \mathrm{~mm}$. The cylinders have a height of $L=927 \mathrm{~mm}$ and an aspect ratio of $\Gamma=L / d=11.9$. For inner cylinder rotation only (the outer cylinder is stationary), the Reynolds number is defined with the velocity of the inner cylinder and the gap width $d$ as

$$
R e_{i}=\frac{\omega_{i} r_{i} d}{\nu} .
$$

Using the viscous velocity $u_{\tau}$ obtained from measurements of the torque, the friction Reynolds number is defined as

$$
R e_{\tau}=\frac{u_{\tau}(d / 2)}{\nu} .
$$

The roughness used was P36 grit sandpaper (VSM, ceramic industrial-grade), that was fixed to the entire surface of the inner cylinder using double-sided adhesive tape (tesa 51970). We define the characteristic length scale of the roughness as $k \equiv 6 k_{\sigma} \approx 1.07 \mathrm{~mm}$ (corresponding to the $99.8 \%$ interval of the height), where $k_{\sigma}$ is the standard deviation of the local roughness height $h(x, y)$ (quantified using confocal microscopy [103] over a square part of the roughness sandpaper with width $25 \mathrm{~mm}$ ), and $k / d=0.014$.

\subsubsection{Experimental procedure}

We performed seven experiments with different rotation rates of the inner cylinder, see table 3.1. During all these experiments, the torque $\mathcal{T}$ that is required to drive the inner cylinder at fixed rotational velocity was measured constantly. The hollow reaction torque sensor that connects the drive shaft to the middle section of the inner cylinder is indicated in figure 3.1(a). By only measuring the torque on the middle section, possible end-plate effects are eliminated [100]. During the torque measurements, PIV was used to obtain the velocity field in the gap. To quantify the reproducibility of our torque measurements, we compared the torque data that were captured during the 


\begin{tabular}{ccccccc}
$T a\left[\times 10^{12}\right]$ & $R e_{i}\left[\times 10^{6}\right]$ & $N u_{\omega}$ & $C_{f}\left[\times 10^{-3}\right]$ & $R e_{\tau}\left[\times 10^{3}\right]$ & $L_{c}^{+}\left[\times 10^{3}\right]$ & $k_{\sigma}^{+}$ \\
\hline 0.31 & 0.46 & 312 & 2.21 & 7.6 & 2.6 & 34 \\
0.57 & 0.62 & 403 & 2.13 & 10.0 & 3.3 & 44 \\
0.92 & 0.78 & 513 & 2.14 & 12.7 & 4.1 & 57 \\
1.47 & 0.99 & 643 & 2.12 & 16.0 & 5.0 & 72 \\
2.18 & 1.20 & 784 & 2.12 & 19.5 & 6.0 & 87 \\
3.55 & 1.54 & 998 & 2.11 & 24.9 & 7.6 & 111 \\
4.71 & 1.77 & 1137 & 2.09 & 28.5 & 8.5 & 127 \\
6.15 & 2.00 & 653 & 1.07 & 23.1 & 6.9 & 0
\end{tabular}

Table 3.1: Control parameters, global response and relevant length scales, measured during the PIV measurements. Ta or $R e_{i}$ characterize the driving of the system. $N u_{\omega}$ is the dimensionless angular velocity flux, $C_{f}$ the friction factor, $L_{c}^{+}$the curvature Obukhov length as defined in equation (3.11) and $k_{\sigma}^{+}$ is the standard deviation of the sandpaper roughness, see [103]. The final row presents the values correponding to the smooth wall measurement of [27]

PIV experiments with three separate torque measurements thereafter. We find a spread in $\mathcal{T}$ smaller than $4 \%$ for all cases. These direct and reproducible measurements of the torque (friction) have an accuracy that is comparable to the measurement accuracy of wall shear stress in flat plate BLs, by means of a drag balance [104].

For the PIV measurements, fluorescent polymer tracer particles

(Dantec FPP-RhB-10 with diameters from $1 \mu \mathrm{m}$ to $20 \mu \mathrm{m}$ ) were added to the working fluid. A horizontal laser sheet of about $1 \mathrm{~mm}$ in thickness illuminated tracer particles in the working liquid at mid-height, through the transparent outer cylinder. The laser sheet was created using a frequency doubled Quantel EverGreen $200 \mathrm{~mJ}$ laser. The fluorescent light emerging from the tracer particles was imaged from below, through a window placed in the bottom plate of the apparatus. For this, a $45^{\circ}$ mirror was positioned under the bottom plate as drawn schematically in figure 3.1. The camera was a high-resolution sCMOS camera (LaVision PCO.edge), with a resolution of $2560 \mathrm{px} \times 2160 \mathrm{px}$ and a pixel size of $6.5 \mu \mathrm{m}$. A $100 \mathrm{~mm}$ focal length objective (Zeiss Makro Planar, $100 \mathrm{~mm}$ ) was used, giving an optical magnification of 0.17.

For each rotational velocity of the inner cylinder, $10^{4}$ image pairs were acquired at a recording frequency equal to the rotation rate. The mean velocity distribution in the horizontal plane was computed using single-pixel ensemble correlation $[105,106]$. The spatial resolution is $50 \mu \mathrm{m}$, leading to about 1600 
independent measurement points in the radial direction, evenly spread over the entire gap. From the correlation function (obtained for every pixel) one can directly extract the standard deviation of the velocity, by integrating the probability density function $\sigma(u)=\int_{-\infty}^{\infty}(u-\langle u\rangle)^{2} \operatorname{PDF}(u) d u$, see [107]. This ensures that all turbulent scales are included in the standard deviation, as opposed to regular PIV analysis. The velocity profiles were smoothed using a Gaussian filter with a standard deviation of $\sigma \approx 0.5 \mathrm{~mm}$.

\subsection{Curvature effects}

\subsubsection{The relative effects of curvature and shear}

To characterize and quantify the relative effects of shear and curvature in TC turbulence, we study the ratio $S$ of turbulence production by shear and curvature related effects $[14,84,97]$

$$
S^{-1}=\frac{\overline{u_{\theta}^{\prime} u_{r}^{\prime}} \frac{d}{d r} U}{\frac{1}{r} \overline{u_{\theta}^{\prime} u_{r}^{\prime}} U}=\frac{1}{\omega} \frac{d U}{d r},
$$

where $u_{\theta}^{\prime}$ and $u_{r}^{\prime}$ are the azimuthal and radial velocity fluctuations, respectively, and $\overline{u_{\theta}^{\prime} u_{r}^{\prime}}$ is the Reynolds stress. The mean azimuthal velocity is denoted by $U$, and $\omega=U / r$ is the mean angular velocity. The curvature Obukhov length $L_{c}$ defined in equation (3.7) for a smooth wall marks the transition from a region where the production of turbulence is dominated by shear $\left(y<0.20 L_{c}\right)$, to a region where it is affected by curvature $\left(y>0.20 L_{c}\right)$. Hence, by this definition, for $S=1$ we have $y^{+}=L_{c}^{+}$. The definition from equation (3.7) builds on the existence of a shear logarithmic region, where the gradient of the mean angular velocity is $\frac{d}{d r} U=u_{\tau} /(\kappa y)$. The angular velocity scale for rough walls is approximated as $\omega=\omega_{i}+\Delta \omega$. Thus the generic curvature Obukhov length $L_{c}$ for smooth and rough walls can be defined with the inner cylinder rotation rate $\omega_{i}$, and the wall-shear stress $\tau_{w}$ only, similar to equation 3.7 , but now for a rough wall,

$$
L_{c}=\frac{u_{\tau}}{\kappa\left(\omega_{i}+\Delta \omega\right)},
$$

so that $L_{c}^{+}\left(\Delta \omega^{+}=0\right)=L_{c, s}^{+}$.

Figure 3.2(a) presents the gradient of the mean angular velocity profile versus $S$, calculated from the PIV results. We find fair collapse of the velocity gradients of smooth (grey) and rough (coloured) wall profiles. When the effects 

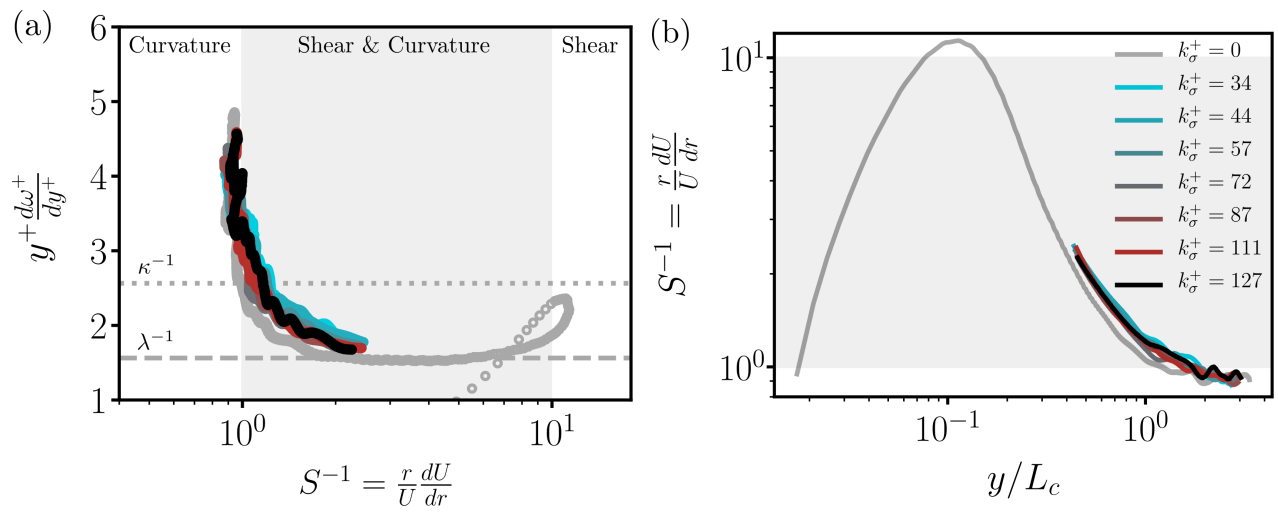

Figure 3.2: (a) Compensated gradient of the mean angular velocity profile versus the ratio $S$ between the turbulence production by shear and that by curvature, see equation (3.10). Dotted and dashed lines represent the slope of the logarithmic velocity profile of the shear and the curvature dominated regimes, $\kappa^{-1}$ and $\lambda^{-1}$, respectively. (b) Ratio $S$ versus the wall-normal distance shifted with the wall offset, $y / L_{c}=\left(r-r_{i}-2 k_{\sigma}\right) / L_{c}$, where $2 k_{\sigma}$ is the approximated wall offset of the sandpaper. Coloured lines are calculated from the PIV data of the rough wall cases. The grey line $\left(k_{\sigma}^{+}=0\right)$ is the smooth wall profile at $T a=6.2 \times 10^{12}\left(R e_{\tau}=23093\right)$, obtained from [27]. 


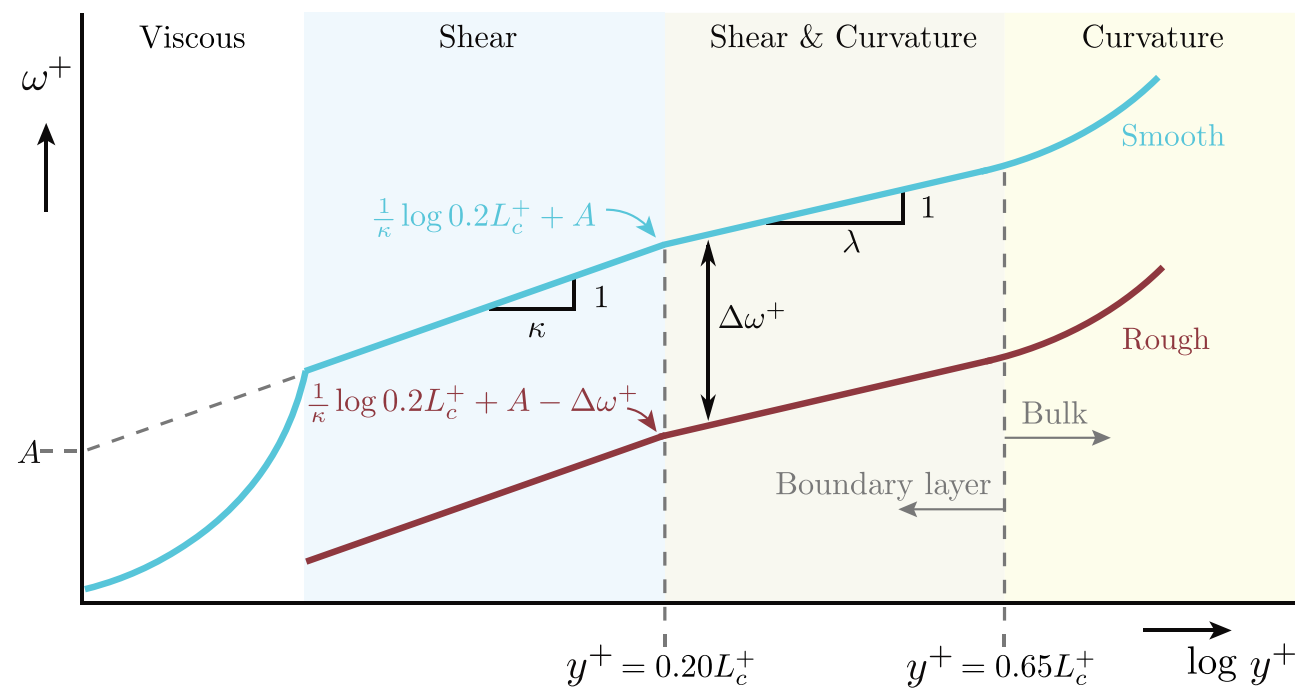

Figure 3.3: Schematic of the various regions in smooth and IC-rough turbulent TC flow at matched $L_{c}^{+}$. The height $y^{+}=0.65 L_{c}^{+}$is defined as the location where the logarithmic profile with slope $\lambda^{-1}$ ends and the constant angular momentum region of the bulk velocity starts.

of curvature are negligible $S \geq \mathcal{O}(10)$, the gradient of the velocity profile approaches $\kappa^{-1} \approx 2.5$. This occurs in a very small region close to the wall, where we cannot measure due to the presence of the sandpaper roughness. For the rough and smooth wall velocity profiles, we find that the gradient approaches $\lambda^{-1}$ in the region where curvature and shear affect the flow. For $S \leq 1$, curvature effects dominate the flow, and a constant angular momentum region (i.e. the bulk flow) sets in [97].

\subsubsection{The mean angular velocity profile}

Figure 3.2(a) shows that the curvature and shear affected region of the BL contains a constant gradient $\left(=\lambda^{-1}\right)$ of the mean angular velocity. From this observation, [97] obtained an equation of the mean angular velocity in the shear and curvature affected region in the BL (in short 'curvature log').

The offset of the logarithmic velocity profile (with slope $\lambda^{-1}$ ) in the curvature and shear affected region, as indicated in figure 3.3, is a function of the wall normal location where curvature related effects impact the flow. From PIV results, the exact location was found to be $y^{+}=0.20 L_{c}^{+}$, with $L_{c}^{+}$defined in 
(3.11). Therefore, the offset is $\kappa^{-1} \log 0.2 L_{c}^{+}+A$, where $A=5.0$ is the offset of the logarithmic velocity profile in the shear affected region [11]. The transition in the logarithmic velocity profile from the shear affected region to the curvature and shear affected region at $y^{+}=0.20 L_{c}^{+}$is not sharp but gradual. To account for this, we introduce a constant $C_{b l}$ that connects the logarithmic velocity profiles of both regions. [97] found that $A+C_{b l}+\left(\frac{1}{\kappa}-\frac{1}{\lambda}\right) \log (0.2)=1.0$, for the inner cylinder, and the mean angular velocity equation, above $y^{+}=0.20 L_{c}^{+}$ as

$\omega^{+}=\frac{1}{\kappa} \log 0.2 L_{c}^{+}+A+C_{b l}+\frac{1}{\lambda} \log \frac{y^{+}}{0.2 L_{c}^{+}}=\frac{1}{\lambda} \log y^{+}+\left(\frac{1}{\kappa}-\frac{1}{\lambda}\right) \log L_{c}^{+}+1.0$,

with $C_{b l}=-3.30$. The transition from the curvature and shear affected region to the constant angular momentum region occurs at $y^{+}=0.65 L_{c}^{+}$. This height we take as our definition of the boundary layer height, above which is the bulk region of constant angular momentum.

\subsubsection{Scale separation}

Key to the understanding of the effects of roughness in TC turbulence is the concept of scale separation. To illustrate this, in figure 3.2(b) we plot $S$ versus the wall-normal distance $y / L_{c}=\left(r-r_{i}-2 k_{\sigma}\right) / L_{c}$. We note that the wall offset $2 k_{\sigma}^{+}$of the rough wall is an approximation. As a reference, we also plot the smooth wall profile (grey) at $\mathrm{Ta}=6.2 \times 10^{12}$ [27], together with the rough wall profiles (colors).

Table 3.1 presents the relevant dynamical length scales in the experiments: namely, $R e_{\tau}, L_{c}^{+}$and $k_{\sigma}^{+}$. The friction Reynolds number $R e_{\tau}$ from equation (3.9) gives the ratio of the largest dynamical length scale in the TC setup to the viscous length scale $\delta_{\nu} . R e_{\tau}$ is of the same order as in the smooth wall TC experiments by [27], where it was $R e_{\tau}=488-23093$, comparable to the rough BL experiments by [108], where $R e_{\tau}=2890-29900$.

The roughness scale in our experiments is much larger than the viscous length scale $\delta_{\nu}$, i.e. $k_{\sigma}^{+}=34-127 \gg 1$, and thus pressure drag dominates over viscous drag. For the flat plate BL experiments of [108] in the fully rough regime, we estimate that $k_{\sigma}^{+}=9-12$ is required, based on the data for which $\Delta U^{+}>8.0$ in [108]. Hence, we are confident that we are indeed far in the fully rough regime. We also find that the roughness sublayer height $\approx 3 k_{\sigma}^{+}$is smaller than the outer bound of the shear dominated logarithmic region, $\approx 0.2 L_{c}^{+}$. For the lowest roughness we have $3 k_{\sigma}^{+} / 0.2 L_{c}^{+}=0.40$, and for the highest roughness 
it is $3 k_{\sigma}^{+} / 0.2 L_{c}^{+}=0.20$. This separation of length scales allows for a region where the logarithmic velocity profile can form. For example in the smooth wall experiments of [27] such a profile was found between $50 \leq y^{+} \leq 600$ for comparable $T a$. We finally find that the outer bound of the curvature dominated logarithmic region $L_{c}^{+}$is smaller than the outer length scale $R e_{\tau}$, so that $0.65 L_{c}^{+} / R e_{\tau} \approx 0.33$. For $y^{+}>0.65 L_{c}^{+}$the curvature dominated bulk, constant angular momentum region, forms. The occurrence and extent of this constant angular momentum region depends on the radius ratio $\eta$, i.e. $L_{c}^{+} / R e_{\tau}$ depends on $\eta$.

Table 1 suggests that the roughness only affects the inertial region where curvature effects are negligible. Hence, we expect that the velocity shift of that region is similar to that of identical sandpaper in a flat plate turbulent BL. In other words, we would expect a fully rough asymptote with slope $\kappa^{-1}$ and a similar value of $k_{s}$ as we would measure for identical sandpaper in a flat plate turbulent BL.

\subsection{Mean velocity profiles of the inner cylinder BL}

In this chapter we will show the angular velocity profile $\omega^{+}\left(y^{+}\right)$rather than the azimuthal velocity profile $u^{+}\left(y^{+}\right)$, as it is $\omega^{+}\left(y^{+}\right)$which is expected, given the arguments based on the Navier-Stokes equations, to have a logarithmic profile [77].

Figure 3.4(a) shows the angular velocity profiles over the rough wall $\omega^{+}=$ $\left\langle\omega_{i}-\omega(r)\right\rangle_{t} / \omega_{\tau}$, with $\omega_{\tau}=u_{\tau} / r_{i}$, versus the wall-normal coordinate $y^{+}$. In this and the next section, we focus our analysis on the mean velocity profiles of the inner cylinder BL, hence $u_{\tau}=u_{\tau, i}$ throughout. In section 3.7 we will report on the bulk profiles. We refer to [97] for an analysis of the smooth velocity profiles of the outer cylinder BL.

Figure 3.4(a) shows that with increasing roughness the rough wall profiles are increasingly shifted downwards, as expected. More importantly, we find from the diagnostic function $y^{+} \frac{d \omega^{+}}{d y^{+}}$(a useful representation of the gradients [11]) in figure 3.4(b), that the slope $\lambda^{-1}$ of the curvature dominated logarithmic region is the same for rough wall TC turbulence as for smooth wall TC turbulence (grey line). Unfortunately, we could not resolve the very thin spatial region where a shear dominated logarithmic was found by [27], as the roughness peaks obstruct the view for the PIV very close to the wall.

For a rough wall, $\Delta \omega^{+}$is a function of the equivalent sand grain roughness height $k_{s}^{+}$and the curvature length $L_{c}$, so that $\Delta \omega^{+}\left(k_{s}^{+}, \frac{k_{s}}{L_{c}}\right)$. When $k_{s} \ll L_{c}$, 

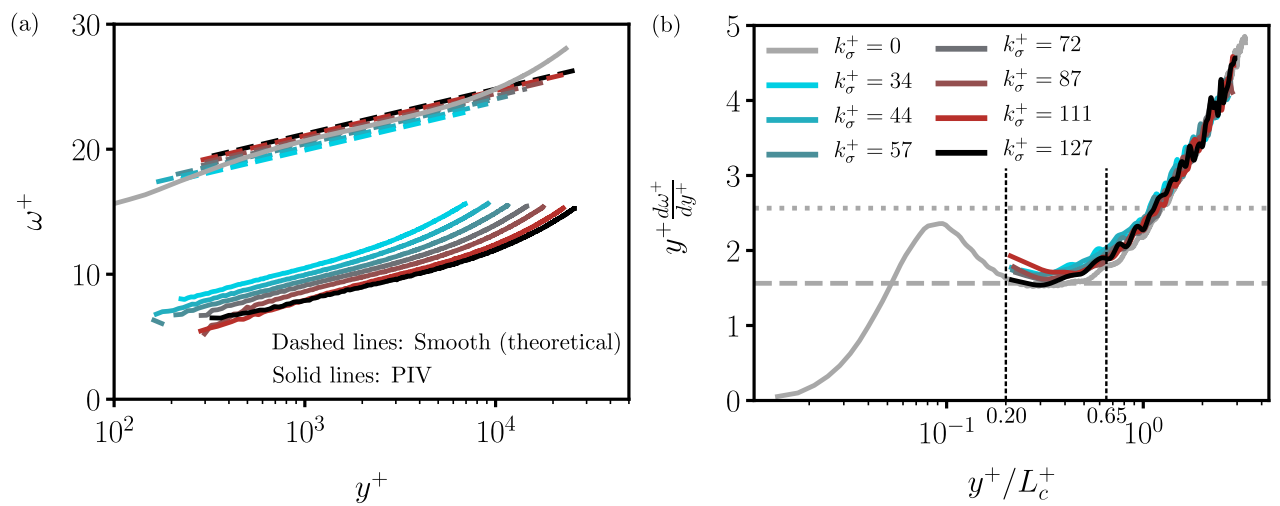

Figure 3.4: (a) Mean angular velocity $\omega^{+}$versus the wall-normal distance $y^{+}$. The solid lines are the measured rough wall profiles. The dashed lines represent the theoretical smooth wall reference profiles (colors are the same), calculated from equation (3.12) and at matching $L_{c}^{+}$and $R e_{\tau}$ with the rough wall profiles. (b) The compensated gradient of the rough wall profiles in (a), where the wall-normal distance is normalized with the curvature length $L_{c}^{+}$. The colors are the same in both figures. The grey line is the smooth wall profile at $T a=6.2 \times 10^{12}$, obtained from [27]. The dashed horizontal line represents the slope $\lambda^{-1}$ of the logarithmic velocity profile in the region where turbulence production is dominated by curvature effects. The dotted horizontal line represents the slope $\kappa^{-1}$ of the logarithmic velocity profile in the region where turbulence production is dominated by shear. 
the angular velocity shift only depends on $k_{s}$, and the shift becomes $\Delta \omega^{+}\left(k_{s}^{+}\right)$. Since the inner cylinder rotates, the plus sign in the denominator of equation (3.11) is connected with the increase of angular fluid velocity in the inner cylinder BL due to the roughness. When we normalize the wall-normal distance with $L_{c}^{+}$, we expect the transition from a curvature logarithmic velocity profile to the constant angular momentum bulk velocity profile to occur at $y^{+} / L_{c}^{+}=0.65$. In figure 3.4(b) we find a fair collapse of both smooth and rough wall profiles in wall-normal direction, when normalized with curvature length $L_{c}^{+}$.

\subsection{The fully rough asymptote}

From the observation that both smooth and rough wall velocity profiles possess the same slope $\lambda^{-1}$ of the curvature logarithmic region (figure $3.4 \mathrm{~b}$ ) we proceed to calculate the angular velocity shift $\Delta \omega^{+}$. Due to the roughness the angular velocity profiles in the shear logarithmic region are shifted, as discussed in section 3.3.1 and illustrated in figure 3.3. This shift remains also in the curvature logarithmic region, where we will now quantify it. The offset of that region scales with $\frac{1}{\kappa} \log \left(0.20 L_{c}^{+}\right)+A$ (figure 3.3 ). Hence, it is imperative to calculate the angular velocity shift from the smooth wall velocity profile at matching $L_{c}^{+}$.

Figure 3.4(a) shows these smooth wall profiles (dashed), where the colors match the respective rough wall cases, and both $L_{c}^{+}$and $R e_{\tau}$ are matched. The velocity shift $\Delta \omega^{+}\left(y^{+}\right)$from the theoretical smooth wall profile, equation (3.12), is plotted in figure 3.5(a). The horizontal plateaus confirm the similarity of the slopes of the velocity profiles. We extract $\Delta \omega^{+}$at $y^{+} \approx 0.4 L_{c}^{+}$ and plot the shift versus the roughness height in figure 3.5(b). When we fit a function of the form $\Delta \omega^{+}=\frac{1}{a} \log k^{+}+b$ through all seven data points, we obtain $a=0.34 \pm 0.02$, to within $15 \%$ of the von Kármán constant $\kappa \approx 0.38$, the slope of the shear dominated logarithmic profile. This confirms our hypothesis, as discussed in section 3.3.1, that the fully rough asymptote for $\delta_{\nu} \ll k<L_{c}^{+}$ has slope $\kappa^{-1}$. For reference, this is much higher than $\lambda^{-1} \approx 0.64^{-1}$, blue line in figure $3.5(\mathrm{~b})$.

To obtain a measure of the equivalent sandgrain roughness height $k_{s}$, we fit the data points to the fully rough asymptote of [10]

$$
\Delta \omega^{+}\left(k_{s}^{+}\right)=\frac{1}{\kappa} \log k_{s}^{+}+5.0-8.5,
$$



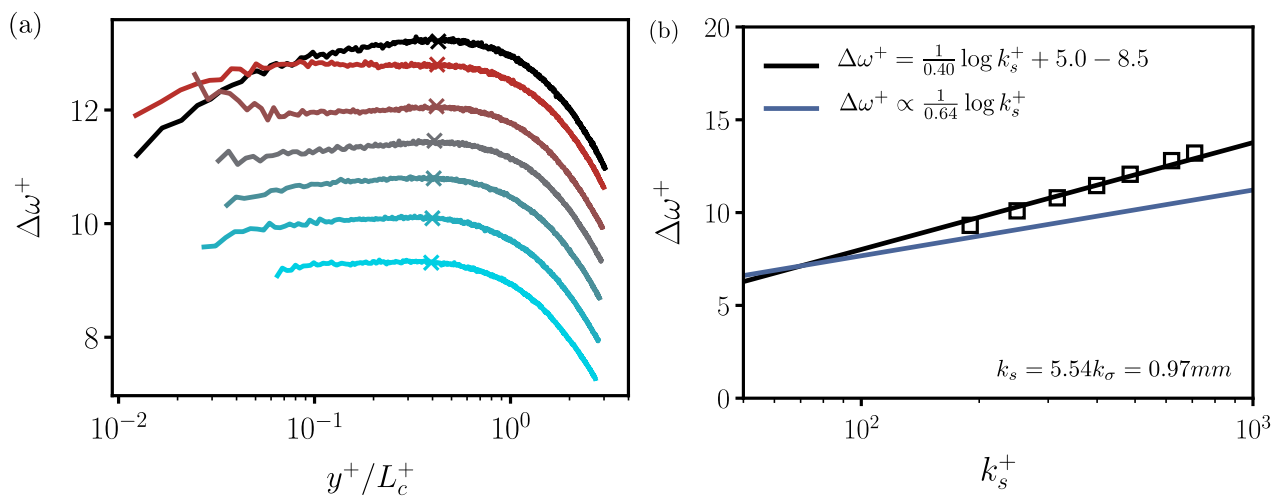

Figure 3.5: $\Delta \omega^{+}$of the rough wall profiles with respect to the reference smooth wall profiles. (a) Velocity shift versus the wall-normal distance $y^{+} / L_{c}^{+}$. (colors the same as figure 3.4) (b) The velocity shift $\Delta \omega^{+}$, crosses in (a), versus the equivalent sand grain height $k_{s}^{+}$. Black symbols are the experimental values. The solid black line is the fully rough asymptote of [10], equation (3.13). The solid blue line is an illustration of the curvature fully rough asymptote, with slope $\lambda^{-1}$ and arbitrary height.

and obtain $k_{s}=5.54 k_{\sigma}=0.97 \mathrm{~mm}$, for $\kappa \approx 0.40$. For reference, the typical grain size is estimated by $6 k_{\sigma}=1.05 \mathrm{~mm}$ [103].

\subsection{The equivalent sand grain roughness height}

The hypothesis in this research, postulated in section 3.3.1, is that the fully rough asymptote in TC turbulence with $\delta_{\nu} \ll k<L_{c}$ is the same (or very similar) to the fully rough asymptote in flows without streamwise curvature. We have already demonstrated in section 3.5 that the slope $\kappa^{-1}$ of the fully rough asymptote is indeed (almost) the same. This leaves us with a comparison of the value of $k_{s}$, between TC turbulence and canonical systems without streamwise curvature.

In literature, we have found two reports on turbulent flows over sandpaper roughness: the work of [108], employing 36 grit sandpaper in a turbulent BL, and [110] who employed (12-, 24-, and 80-) grit sandpaper in turbulent BL flow. In the rough wall TC experiments reported here we used grit 36 sandpaper. However, it is essential to realize that sandpaper is not only defined by the grit size. Other statistics, like the skewness (an important parameter [98], which is 0.93 here, and only 0.09 in [108]), do vary with manufacturing meth- 


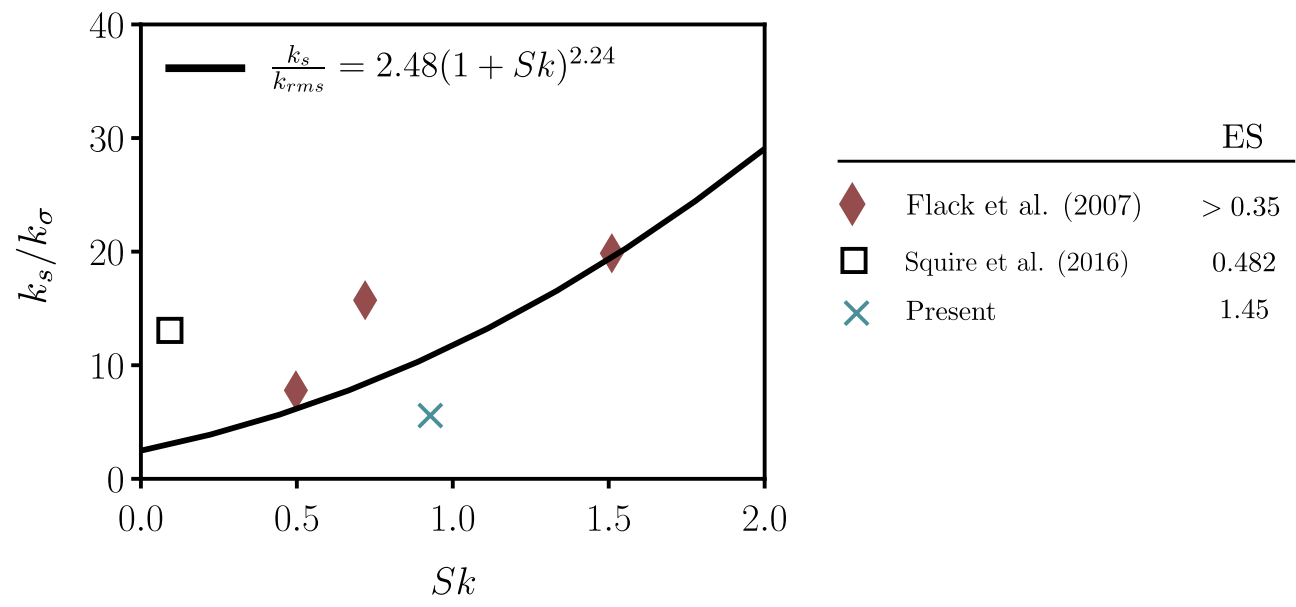

Figure 3.6: Relationship between the equivalent sand grain roughness height divided by the root-mean-square height $k_{s} / k_{\sigma}$, and the skewness parameter $S k$ of different sandpaper surfaces. The solid black line is the empirical correlation for $S k>0$ from [109]. Data from turbulent boundary layer flow using grit (12, 24, and 80) sandpaper [110], of which the surface statistics are listed in [23], turbulent boundary layer using grit 36 [108] and turbulent TC flow using grit 36 (present). ES is the effective slope defined as $\left|\overline{\frac{d k}{d(r \theta)} \mid}\right|$ [30]. 
ods. We have tried to use the very same sandpaper type (SP40F, Awuko Abrasives) as [108]. Unfortunately, the sandpaper turned out to be not waterproof, and detached from the inner cylinder. We then applied new water resistant sandpaper (VSM, P36 grit ceramic industrial grade), with different surface roughness statistics.

To compare the drag property of the sandpaper surfaces in TC, to the respective sandpaper surfaces in literature, we plot the relationship between $k_{s}$ and the root-mean-square and skewness in figure 3.6. The surface properties of the sandpaper surface from [110] are taken from [23]. The solid black line is the empirical correlation from [109]. We find that the relation between $k_{s}$ and the Skewness $S k$ and the root-mean-square height $k_{r m s}$ of sandpaper used in our rough wall TC experiments is consistent with the empirical trend given for the sandpaper used in rough wall turbulent BL flow analysis. Whether, the deviation originates from the difference between TC and canonical systems without curvature, or originates from the different surface statistics (e.g. the ES for the present surface is higher, indicating a denser surface), remains to be resolved.

\subsection{The constant angular momentum region}

Thus far, we have discussed the velocity profiles of the inner cylinder boundary layer, i.e. $y^{+}<0.65 L_{c}^{+}$. By means of matching this profile to the bulk velocity profile at boundary layer height, one can derive the relationship between the torque $N u_{\omega}(T a)$ and the velocity of the inner cylinder [15,97]. For smooth wall inner cylinder rotating turbulent TC flow, it is well known that the angular momentum in the bulk $\left(M_{b}\right)$ is constant $[84,89]$, and, in fact, very close to half the inner cylinder angular momentum $\left(M_{i}=\omega_{i} r_{i}^{2}\right), M_{b}=0.5 M_{i}$, for stationary outer cylinder. For rough wall TC flow however, and especially for asymmetric roughness when the inner cylinder is of a different roughness height than the outer cylinder, the exact value of $M_{b}$ is a priori unknown. However, what was shown is that for very rough walls the bulk azimuthal velocity profile is shifted towards the rough cylinder, due to the stronger coupling to that side thanks to the roughness $[42,43,96]$.

If the bulk region velocity conforms to a constant angular momentum, it should match the angular momentum at the edge of the BL $r=r_{i}+\delta_{r}$, where $\delta_{r}=0.65 L_{c}$. The momentum ratio $\left(M_{b} / M_{i}\right)$ is the angular momentum in the 

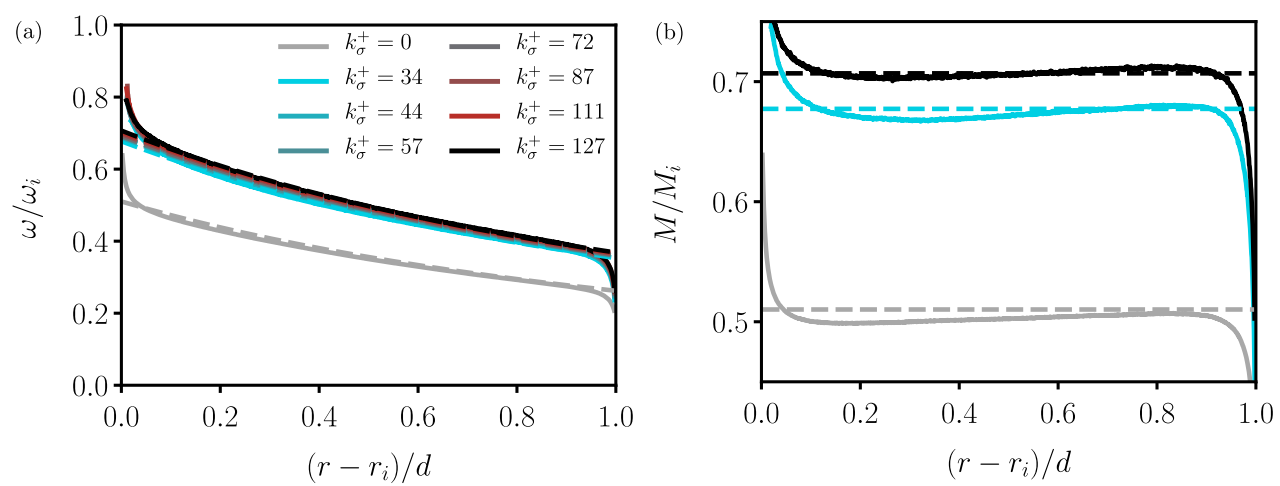

Figure 3.7: Bulk velocity profiles. (a) The mean angular velocity normalized with the inner velocity $\omega / \omega_{i}$, versus the radius $\left(r-r_{i}\right) / d$ normalized with the gap width $d$. The profiles for different roughness heights $k_{\sigma}^{+}$are compared. The bulk profile is strongly shifted towards the rough inner cylinder, as the roughness there enhances the coupling between the inner BL and the bulk, similarly as the ribs have done in [43]. (b) The angular momentum $M$, normalized with the inner cylinder angular momentum $M_{i}$. Solid lines are the PIV results and dashed lines $\left(M_{b} / M_{i}\right)$ are calculated from equation (3.14). The colors are the same in both figures. The grey line is the smooth wall profile at $T a=6.2 \times 10^{12}$, obtained from [27]. 
bulk over the angular momentum of the inner cylinder

$$
\frac{M_{b}}{M_{i}}=\frac{\left.\omega\right|_{y=\delta_{r}}\left(r_{i}+\delta_{r}\right)^{2}}{\omega_{i} r_{i}^{2}},
$$

where $\left.\omega\right|_{y=\delta_{r}}=\omega_{\tau, i}\left(\omega_{i}^{+}-\omega_{r}^{+}\left(y^{+}=\delta_{r}^{+}\right)\right)$, and we use the velocity profile of the rough inner cylinder BL, figure 3.3 and $(3.12)-\Delta \omega^{+}, \omega_{r}^{+}\left(y^{+}=\delta_{r}^{+}\right)=$ $\frac{1}{\lambda} \log \delta_{r}^{+}+\left(\frac{1}{\kappa}-\frac{1}{\lambda}\right) \log L_{c, r}^{+}+1.0-\Delta \omega^{+}$. Figure 3.7 compares the result from equation (3.14) (dashed line) with the experimentally obtained velocity profiles (solid lines), demonstrating agreement between the calculated and the measured profiles. This supports the assumption that also the rough-wall velocity profiles conform to a constant angular momentum in the bulk. Finally, we point out that the 'overshooting' of the profiles in the bulk, i.e. the slight increase in $M$ with increasing $r$, is likely an effect of the turbulent Taylor vortices, and is therefore expected to depend on the height coordinate $z$ [101]. It is due to the detaching plumes which are transported to the other side of the gap by the Taylor rolls. Similar overshooting is well known from temperature profiles in turbulent Rayleigh-Bénard flow [111,112]

\subsection{Calculation of $\mathrm{Nu}(\mathrm{Ta})$ and $\mathrm{C}_{\mathrm{f}}\left(\mathrm{Re}_{\mathrm{i}}\right)$}

Since the angular momentum in the bulk is to a good approximation constant, we can match the angular momentum of the inner cylinder BL at BL height with the angular momentum of the outer cylinder BL at BL height, i.e. $M\left(\delta_{i, r}\right)=M\left(\delta_{o, s}\right)$. This approach is based on the matching of BL and bulk velocity profiles in the recent CPS model [15]. Subscripts $(i, o)$ refer to inner cylinder and outer cylinder BL quantities, where subscripts $(s, r)$ refer to smooth and rough wall quantities, and $\delta=0.65 L_{c}$ for inner cylinder and outer cylinder (rough and smooth) so that $\delta_{i, r}^{+}=\alpha L_{c, i, r}^{+}, \delta_{o, s}^{+}=\alpha L_{c, o, s}^{+}$with $\alpha=0.65$. The matching argument becomes

$$
\left(r_{i}+\delta_{i, r}\right)^{2} \omega_{\tau, i} \omega_{I C}^{+}\left(\delta_{i, r}^{+}\right)=\left(r_{o}-\delta_{o, s}\right)^{2} \omega_{\tau, o} \omega_{O C}^{+}\left(\delta_{o, s}^{+}\right),
$$

where we realize that $\omega_{\tau, o}=\eta^{2} \omega_{\tau, i}$. We substitute the BL equations for respectively rough and smooth walls into equation (3.15) and obtain

$$
\begin{gathered}
\left(r_{i}+\delta_{i, r}\right)^{2} \omega_{\tau, i}\left(\omega_{i}^{+}-\frac{1}{\lambda} \log \left(\delta_{i, r}^{+}\right)-\left(\frac{1}{\kappa}-\frac{1}{\lambda}\right) \log \left(L_{c, i, r}^{+}\right)-C_{i}+\Delta \omega^{+}\right)= \\
\left(r_{o}-\delta_{o, s}\right)^{2} \omega_{\tau, o}\left(\frac{1}{\lambda} \log \left(\delta_{o, s}^{+}\right)+\left(\frac{1}{\kappa}-\frac{1}{\lambda}\right) \log \left(L_{c, o, s}^{+}\right)+C_{o}\right) .
\end{gathered}
$$


The rough wall, inner cylinder BL height $\delta_{i, r}^{+}$, and the velocity shift $\Delta \omega^{+}$are functions of the sand grain size $k_{s}^{+}$. This makes the matching equation more involved, in comparison to the smooth wall case [15,97].

Following [15], we now rewrite the equation in terms of $R e_{\tau, i}$ and $R e_{i}$. The inner cylinder angular velocity becomes

$$
\omega_{i}^{+}=\frac{R e_{i}}{2 R e_{\tau, i}} .
$$

The equivalent sand grand size is

$$
k_{s}^{+}=2 \frac{k_{s}}{d} R e_{\tau, i}=\epsilon R e_{\tau, i} .
$$

The fully rough asymptote from equation (3.13) can now be rewritten as

$$
\Delta \omega^{+}=\frac{1}{\kappa} \log \left(\epsilon R e_{\tau, i}\right)+A-B .
$$

The inner cylinder, rough wall, $\mathrm{BL}$ height $\delta_{i, r}^{+}$is rewritten from $\delta_{i, r}^{+}=\alpha L_{c, i, r}^{+}$ as

$$
\delta_{i, r}^{+}=\frac{2 \alpha \eta R e_{\tau, i}}{\kappa(1-\eta) \mathcal{Z}} ; \quad \text { with } \quad \mathcal{Z}=\left(\frac{R e_{i}}{2 R e_{\tau, i}}+\frac{1}{\kappa} \log \left(\epsilon R e_{\tau, i}\right)+A-B\right) .
$$

The outer cylinder, smooth wall, BL height $\delta_{o, s}^{+}$is rewritten from $\delta_{o, r}^{+}=\alpha L_{c, o, r}^{+}$ as

$$
\delta_{o, s}^{+}=\frac{4 \alpha \eta^{2} R e_{\tau, i}^{2}}{\kappa(1-\eta) R e_{i}}
$$

We can now substitute equations (3.17)-(3.21) into (3.16), and obtain

$$
\begin{gathered}
\left(1+\frac{\alpha}{\kappa \mathcal{Z}}\right)^{2}\left(\frac{R e_{i}}{2 R e_{\tau, i}}-\frac{1}{\lambda} \log \left(\frac{2 \alpha \eta R e_{\tau, i}}{\kappa(1-\eta) \mathcal{Z}}\right)\right. \\
\left.-\beta \log \left(\frac{2 \eta R e_{\tau, i}}{\kappa(1-\eta) \mathcal{Z}}\right)+\frac{1}{\kappa} \log \left(\epsilon R e_{\tau, i}\right)+A-B-C_{i}\right)= \\
\left(1-\frac{2 \alpha \eta R e_{\tau, i}}{\kappa R e_{i}}\right)^{2}\left(\frac{1}{\lambda} \log \left(\frac{4 \alpha \eta^{2} R e_{\tau, i}^{2}}{\kappa(1-\eta) R e_{i}}\right)+\beta \log \left(\frac{4 \eta^{2} R e_{\tau, i}^{2}}{\kappa(1-\eta) R e_{i}}\right)+C_{o}\right),
\end{gathered}
$$

where $\beta=\left(\frac{1}{\kappa}-\frac{1}{\lambda}\right)$. This implicit equation can be solved numerically to obtain $\operatorname{Re}_{\tau, i}\left(R e_{i}\right)$ with parameters $C_{i}=1.0, C_{o}=2.0, A=5.0, B=8.5, \kappa=$ 

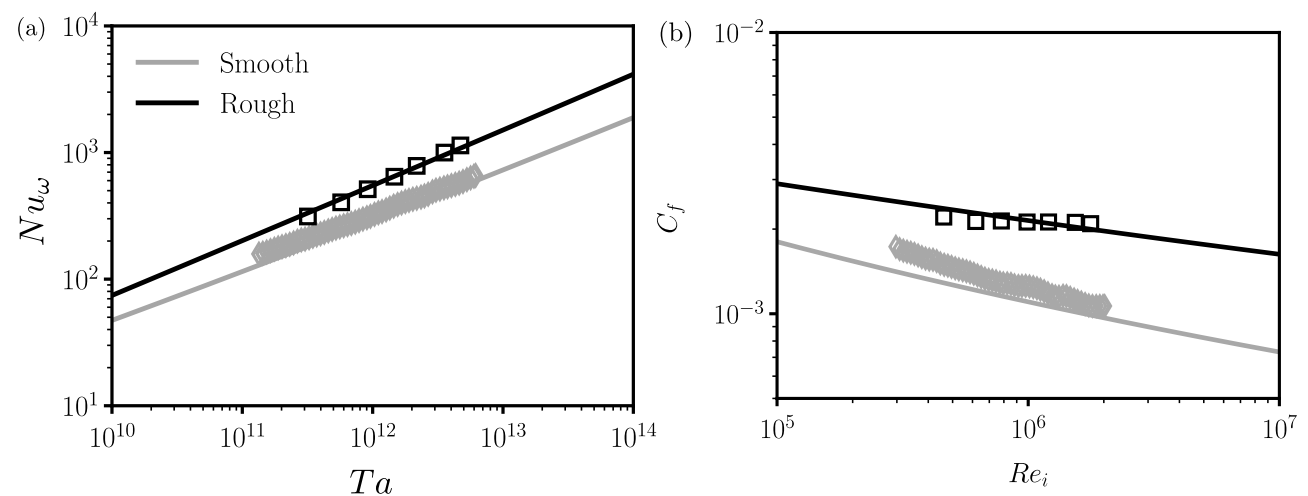

Figure 3.8: Global response of the rough $k_{s} / d=0.012$ and smooth wall TC turbulence. (a) $N u_{\omega}=\frac{2 R e_{\tau}^{2} \eta(1+\eta)}{R e_{i}}$ versus $T a$. (b) Friction factor $C_{f}=\frac{8 R e_{\tau}^{2}}{R e_{i}^{2}}$ versus $R e_{i}$. Black diamonds are the smooth wall experiments of [94], where the solid black line shows the theory of [97] for smooth wall TC turbulence. Blue squares are the rough inner cylinder measurements from the present work. The solid blue line is equation (3.22).

$0.39, \lambda=0.64, \alpha=0.65$ for these experiments, $\eta=0.714$ and $\epsilon=2(0.9694 / 80)$. Finally, by means of equations (3.3) - (3.8), we express the result $R e_{\tau, i}\left(R e_{i}\right)$ into $N u_{\omega}(T a)$ and $C_{f}\left(R e_{i}\right)$ respectively.

Figure 3.8 presents the final result, together with the experimental data from smooth walls [94] and with the equation for smooth wall TC [97] (grey). The black open squares represent the fully rough inner cylinder rotating TC experiments presently. The black solid line is our calculation from equation (3.22). We emphasize that no fitting parameters are used. All parameters find their origin in the velocity profiles, and originate from the slopes of the logarithmic velocity profiles $\left(\kappa^{-1}, \lambda^{-1}\right)$, the offset of the smooth velocity profile $\left(A, C_{i}, C_{o}\right)$ or the BL thickness fit for smooth walls $\alpha$. This reflects that all parameters are universal for all radius ratios, and cannot be 'tuned'.

The agreement between equation (3.22) and the experimental data (the maximum error is only $\approx 5 \%$ ) is convincing. It implies that from straightforward measurements of the torque, for given inner cylinder rotation speed, we can calculate the value of $k_{s}$ with a reasonable accuracy. This means that the TC facility can potentially be used for direct, fast, measurements of surface drag properties, as characterized by $k_{s}$. 


\subsection{Summary, conclusions, and outlook}

We carried out experiments of inner cylinder rotating (and stationary outer cylinder) Taylor-Couette (TC) turbulence with a rough inner cylinder and a smooth outer cylinder. We measured the torque, and, by means of PIV, the mean angular velocity profiles. The rough surface consisted of P36 industrial grade sandpaper, where the roughness height $k=6 k_{\sigma}$, with $k_{\sigma}$ the standard deviation of the roughness height, over the gap width $d$ was $k / d=0.014$. The roughness height $k$ was much larger than the viscous length scale $\delta_{\nu}$, such that $k / \delta_{\nu}=204-762$. The velocity shift of the rough wall azimuthal velocity profiles was, compared to the reference smooth wall, in the log-law region $\Delta \omega^{+}>9$ over the whole range of $4.6 \times 10^{5}<\operatorname{Re}_{i}<1.77 \times 10^{6}$. Hence the sandpaper was hydrodynamically fully rough. Furthermore, the roughness height $k_{\sigma}<0.015 L_{c}$, where $0.20 L_{c}$ is the height [97] which separates the region in the $\mathrm{BL}$ where production of turbulence is dominated by shear, and the region in the BL where production of turbulence is affected by effects of streamwise curvature.

Using the mean azimuthal velocity profiles, we found that the slope of the fully rough asymptote, characterized by $\kappa=0.34 \pm 0.02$, was similar to previous findings in flat plate BLs $\kappa \approx 0.38$. Also, the value of the equivalent sand grain roughness height $k_{s}$ compared reasonably well with those found for sandpaper in flat plate BLs $[108,110]$.

Finally, to obtain the relationship between the dimensionless torque and dimensionless driving of the system $N u_{\omega}(T a)$, we employed a matching argument between the inner cylinder BL rough mean angular momentum profile at the inner cylinder BL height, and the smooth outer cylinder BL mean angular momentum profile, at the outer cylinder BL height, based on the CPS model of [15], see also [97]. To justify this, we first showed that for a rough wall inner cylinder, a region of constant angular momentum exists in the bulk. We find a convincing overlap between the calculated value of the torque (or wall shear stress), and the experimentally measured values of the torque, with a maximum error of $\approx 5 \%$.

These findings indicate that the turbulent TC facility can be a valuable setup for characterizing the drag properties of any rough surface. Direct and straightforward measurements of the torque can now be translated to a value of the equivalent sand grain roughness height $k_{s}$. It seems that the value of $k_{s}$ found in TC is similar to the value of $k_{s}$ found in flat plate BLs.

In outlook of future work, we propose that more studies in both turbulent flat 
plate BLs and turbulent TC flow, with identical rough surfaces, are carried out to further compare the drag properties of these surfaces. Further unanswered questions include the effects of even more considerable roughness penetrating the curvature affected logarithmic regime of the $\mathrm{BL}$, which is related to finding the slope of the fully rough asymptote in that region. This could also be achieved by employing a TC setup with a lower radius ratio $\eta$, thus increasing curvature effects. 


\section{Chapter 4}

\section{Controlling secondary flow in Taylor-Couette turbulence through spanwise-varying roughness}

Highly turbulent Taylor-Couette flow with spanwise-varying roughness is investigated experimentally and numerically (direct numerical simulations (DNS) with an immersed boundary method (IBM)) to determine the effects of the spacing and spanwise width $s$ of the spanwise varying roughness on the total drag and on the flow structures. We apply sandgrain roughness, in the form of alternating rough and smooth bands to the inner cylinder. Numerically, the Taylor number is $\mathcal{O}\left(10^{9}\right)$ and the roughness width is varied between $0.47 \leq \tilde{s}=s / d \leq 1.23$, where $d$ is the gap width. Experimentally, we explore $\mathrm{Ta}=\mathcal{O}\left(10^{12}\right)$ and $0.61 \leq \tilde{s} \leq 3.74$. For both approaches the radius ratio is fixed at $\eta=r_{i} / r_{o}=0.716$, with $r_{i}$ and $r_{o}$ the radius of the inner and outer cylinder respectively. We present how the global transport properties and the local flow structures depend on the boundary conditions set by the roughness spacing $\tilde{s}$. Both numerically and experimentally, we find a maximum in the angular momentum transport as function of $\tilde{s}$. This can be attributed to the re-arrangement of the large-scale structures triggered by the presence of the rough stripes, leading to correspondingly large-scale turbulent vortices.

Published as: D. Bakhuis, R. Ezeta, P. Berghout, P.A. Bullee, N.C. Tai, D. Chung, R. Verzicco, D. Lohse, S.G. Huisman, and C. Sun, Controlling secondary flow in Taylor-Couette turbulence through spanwise-varying roughness, J. Fluid Mech. 883, A15 (2020).

D. Bakhuis, R. Ezeta and P. Berghout are shared first author. 


\subsection{Introduction}

In nearly all industrial applications and geophysical flows, turbulence is partly or completely wall-bounded. In general, boundaries are not smooth but their surface is rather irregular and rough. Accordingly, such flows are extensively studied, although mainly under the approximation that the roughness is homogeneous [6]. Homogeneously rough surfaces have a characteristic length scale $k$ that is much smaller than the largest wall normal length scale $\delta$. The effects of the roughness in these flows is believed to be confined to the immediate vicinity of the wall (i.e. the roughness sublayer), whereas in the outer, inertial layer, the flow only experiences the effective shear stress of the surface (Townsend's outer layer similarity, [4]). As such, the focus of many studies is to find functional relationships between the parameters describing the roughness geometry and the skin friction coefficient $C_{f}$ [23]. In practice, however, flows are bounded by rough boundaries that, not only vary on the scale of $k$, but also on a much larger scale $s$, being $s=\mathcal{O}(\delta)$. Whereas these variations can occur either laterally (spanwise) or longitudinally (streamwise), we focus here only on the former. Such examples are found in shipping (i.e. the formation of stripes of bio-fouling on ship hulls [20]) and geophysical flows (e.g. the atmospheric flows over spanwise-varying terrain [113]).

Hitherto, the research is focused on the effects of spanwise-varying rough surfaces on canonical systems of wall-bounded turbulence, i.e. pipe- [114], boundary layer- [115], and channel-flow [116]. The hallmark of flows over these surfaces is the presence of spanwise wall-normal secondary flows of size $\mathcal{O}(\delta)$, with mean streamwise vorticity. Examples of studies where this has been observed are the works of [114] on the effects of convergent and divergent grooves (reminiscent of shark skin) and the work by [117] on spanwise-varying riverbeds. We note that earlier research dates back to the works of $[118,119]$ in the field of surface stress variations in duct flows.

Following up on the work of [114], [120] set out to perform a parametric study of the converging-diverging riblets surface in a zero pressure gradient boundary layer (BL). They found a thickening of the BL height above the converging regions, and vice versa above the diverging regions. Furthermore, the energy spectra showed an increased energy content of the larger scales. [121] performed stereo particle image velocimetry (PIV) in the spanwise wall-normal plane for the flow over a turbine blade replica and found spanwise variations of the order $\delta$ in the mean velocity field. Within the same configuration, [122] identified regions of low momentum pathways (LMPs) and high momentum 
pathways (HMPs) in the instantaneous fields. Here, LMPs coincide with regions of enhanced turbulent kinetic energy (TKE) and Reynolds shear stress (RSS), and rather remarkably, these regions do seem to occur at recessed roughness heights. [123] found very similar behaviour of the secondary flows for a much more regular surface geometry. [124] found that only when $s / \delta \gtrsim 0.5$, secondary flow formation is observed. Here $s$ is the spacing between streamwise aligned Lego ${ }^{\circledR}$ blocks. For $s / \delta \lesssim 0.5$ the secondary flows are confined to the roughness sublayer. Interestingly, contrary to the findings of [122], they found LMPs on top of their elevated blocks, and HMPs in between the roughness strips. [125], however, found $s / H \gtrsim 0.2$, with $H$ the channel half height, as the threshold for heterogeneous behaviour of the streamwise aligned pyramid elements.

It is an open question as to why the varying experiments in literature show conflicting orientations of the secondary flow. We note that there are some differences, namely that the experiments in the paper by [124] consist of small strips of elevated roughness, and wide regions of recessed roughness heights. Rather than alternating rough and smooth strips of equal width. A hypothesis concerns the location of the virtual origin of the rough part, with respect to the smooth part. This could be either below (recessed), or above (protruding) the smooth surface, resulting in either an inflow (recessed) or an outflow (protruding) region above the roughness.

By carefully assessing the terms in the transport equation TKE, [115] found that spanwise variations of roughness lead to a local imbalance of production and dissipation of TKE, as already proposed by [118]. Since the secondary flows are driven by a spatial gradient of the RSS, they find that the mean secondary flows are Prandtl's secondary flow of the second kind [126]. [127] observed a breakdown of outer layer similarity in the local profiles of the mean flow, turbulent intensity, and the energy spectra, evidently induced by the presence of the secondary vortices. Finally, [116] studied the influence of the spacing of idealized (i.e. no geometric induced disturbances to the flow) regions of low shear stress and high shear stress. They found that for $s / \delta \lesssim 0.39$ the notion of outer layer similarity is retained. Interestingly, for $s / \delta \gtrsim 6.28$, they found a sign reversal of the isovels (stream velocity contour lines), with respect to the orientation of the secondary flows, that remain upwelling over low shear stress regions.

The aforementioned studies were all carried out in systems that lack two features which are intrinsic to many applications, namely the curvature in the streamwise direction (as in turbine blades), and the presence of strong sec- 
ondary motions (as in the atmospheric boundary layer). Especially the application towards turbine blades is interesting, as also studied by [121]. Here we come closer to the application by studying the turbulent flow over curved, rotating walls, present in the turbines.

\subsubsection{Taylor-Couette flow}

A canonical system in which these two properties can be observed simultaneously is the Taylor-Couette (TC) flow. TC flow is the flow between two coaxially, independently rotating cylinders. Its geometry is characterized by two dimensionless parameters; the radius ratio $\eta=r_{i} / r_{o}$ and the aspect ratio $\Gamma=L / d$. Here, $r_{i}$ and $r_{o}$ are the inner cylinder radius and outer cylinder radius respectively. $L$ is the axial length of the inner cylinder, and $d=r_{o}-r_{i}$ the gap between the two cylinders. Since TC is a closed system, one can directly relate global and local quantities through exact mathematical relations [48]. The driving strength in TC flow is expressed in dimensionless form by the Taylor number:

$$
\mathrm{Ta}=\frac{1}{4} \sigma d^{2} \frac{\left(r_{i}+r_{o}\right)^{2}\left(\omega_{i}-\omega_{o}\right)^{2}}{\nu^{2}}
$$

where $\omega_{i}$ and $\omega_{o}$ are the inner and outer angular velocity of the cylinders, respectively, $\nu$ is the kinematic viscosity of the fluid, and $\sigma=((1+\eta) /(2 \sqrt{\eta}))^{4}$ is the so-called geometric Prandtl number, in analogy to the Prandtl number in Rayleigh-Bénard convection [48]. Alternatively, when the outer cylinder is at rest $\left(\omega_{o}=0\right)$, the driving strength can also be expressed with a Reynolds number based on the inner scales $\operatorname{Re}_{i}=r_{i} \omega_{i} d / \nu$. This Reynolds number and Ta $\left(\omega_{o}=0\right)$, are related by $\operatorname{Re}_{i}=\left(8 \eta^{2} /(1+\eta)^{3}\right) \sqrt{\mathrm{Ta}}$. In TC flow, the angular velocity flux $J^{\omega}$ is radially conserved ( [48]), where effects of top and bottom plates are assumed negligible. Here, $J^{\omega}=r^{3}\left(\left\langle u_{r} \omega\right\rangle_{A, t}-\nu \frac{\partial}{\partial r}\langle\omega\rangle_{A, t}\right)$, where the brackets $\langle\cdot\rangle_{A, t}$ denote averaging over a cylindrical surface and time. The angular momentum flux for the case of laminar flow is $J_{\text {lam }}^{\omega}=2 \nu r_{i}^{2} r_{o}^{2}\left(\omega_{i}-\right.$ $\left.\omega_{o}\right) /\left(r_{o}^{2}-r_{i}^{2}\right)$. In this way the response of the flow is quantified by the dimensionless Nusselt number $\left(\mathrm{Nu}_{\omega}\right)$, which is also directly related to the torque $\mathcal{T}$ that is required to drive the cylinders at constant speed, i.e.

$$
\mathrm{Nu}_{\omega}=\frac{J^{\omega}}{J_{\text {lam }}^{\omega}}=\frac{\mathcal{T}}{2 \pi L \rho J_{\text {lam }}^{\omega}} .
$$

Here, $\rho$ is the density of the working fluid. Alternatively, the torque of the system can be non-dimensionalized to form the friction coefficient $C_{f}=$ 
$\mathcal{T} /\left(\rho L \nu^{2} \operatorname{Re}_{i}^{2}\right)$, which is directly related to the Nusselt number:

$$
\mathrm{Nu}_{\omega}=C_{f} \omega_{i}\left(r_{o}-r_{i}\right)^{2}\left(r_{o}^{2}-r_{i}^{2}\right) /\left(4 \pi \nu r_{o}^{2}\right) .
$$

The inner friction velocity $u_{\tau, i}$ is also related to the torque by $u_{\tau, i}=\sqrt{\mathcal{T} /\left(2 \pi r_{i}^{2} \rho L\right)}$, which is used to scale quantities in the inner layer, indicated with the superscript ' + '. The friction angular velocity $\omega_{\tau}$ equals $u_{\tau} / r$. Lastly, a frictional Reynolds number based on the inner scales can be defined as $\operatorname{Re}_{\tau}=u_{\tau, i} d /(2 \nu)$. Secondary flows are featured in TC flow, in the form of large scale vortices with a mean streamwise vorticity component, the so-called Turbulent Taylor Vortices (TTV). These structures are reminiscent of laminar Taylor vortices, which transition through a series of instabilities into turbulence once the flow becomes unstable [12]. As noted by [128], the axial wavelength $\lambda / d$ of the TTVs, i.e. the distance between two rolls, is primarily a function of $\eta$ and Re. When Re is large enough $\left(\mathcal{O}\left(10^{6}\right)\right)$, the rolls are observed to persist in the system [101]. Here, multiple states for $\eta=0.716$ can be observed in a certain regime of counter-rotating cylinders, namely $a \in[0.17,0.51]$, where $a=-\omega_{o} / \omega_{i}$ is their rotation ratio. These multiple states are characterized by a change in the number of rolls present in the system and, as a consequence, in their averaged axial wavelength $(\lambda / d=1.46$ or $\lambda / d=1.96)$. These states, with the transition between them being strongly hysteretic, even at $\operatorname{Re}=\mathcal{O}\left(10^{6}\right)($ $[101,129,130])$, result in different torques for the same rotation rates, which reflects the importance of the large scale structures (TTV) in transporting angular momentum. At pure inner cylinder rotation however $(a=0)$, no multiple states are found and the rolls are observed to be less coherent and stable. Finally, we note that the effect of the curvature of the cylinders is quantified by the radius ratio $\eta$, and it has a tremendous impact on the flow organization as was reported by $[93,131]$. For a detailed review on turbulent Taylor-Couette flow we refer the reader to the review from Grossmann et al. (2016).

Roughness in a TC geometry has been studied in various ways: $[39,40]$ used obstacle roughness, in the form of axial riblets, to study the scaling of the angular momentum transport with the driving strength. [41] investigated the influence of grooves for large $\mathrm{Ta}\left(\mathcal{O}\left(10^{10}\right)\right)$, and found that at the tips of the grooves, plumes are preferentially ejected. In a more recent work, [43] found that by using a similar configuration of rough walls as [40], the scaling $\mathrm{Nu}_{\omega} \propto \mathrm{Ta}^{1 / 2}$, where the logarithmic corrections of the ultimate regime vanish for asymptotically high Reynolds numbers, is obtained. This regime is referred to as the asymptotic ultimate regime $([13,43])$. They attribute this to a 
dominance of the pressure drag over the viscous drag on the cylinders. Very recently, [96] studied the influence of sandgrain roughness in TC flow, and found similarity of the roughness function with the same type of roughness in pipe flow [10]. None of the TC papers described above reported an influence of the roughness variations in the axial direction, i.e. the spanwise direction. In this manuscript we will fill this gap and study the effects of spanwisevarying roughness in highly turbulent TC flow with Ta up to $\mathcal{O}\left(10^{12}\right)$, for the case of pure inner cylinder rotation $a=0$, where secondary flows are present in the form of TTVs. In particular, we focus on the effect of spanwise-varying roughness on the TTVs and thus, on the global and local response of the flow. We introduce the roughness through a series of stripes which extend along the entire circumference of the inner cylinder (IC). This gives rise to a spanwise (axial) arrangement of roughness which we characterize with the widths of the roughness stripe. We conduct both, experiments and direct numerical simulations (DNS) for various dimensionless stripe widths $\tilde{s}=s / d$, i.e. the width of the roughness stripe normalized with the gap width. For practical reasons, the roughness area is slightly larger than the smooth area, with a rough area coverage of $56 \%$.

The structure of the manuscript is as follows. In section 4.2 , we introduce the experimental and numerical methods. In section 4.3 .1 we show the local response of the flow due to the varying roughness arrangement. In section 4.3.2, we study its effect on the global quantities. In section 4.3.3, we link the global and local observations and explain the physical mechanism between the interaction of the rolls and the roughness. We finalize the manuscript in section 4.4 with some conclusions and an outlook to future work.

\subsection{Methods}

\subsubsection{Numerical methods}

The radius ratio $\eta=0.714$, the same as in experiments. The streamwise and spanwise lengths of the computational domain are set to match the minimum computational domain size as studied in [46]. As such, we simulate aspect ratios of $2.0 \leq \Gamma \leq 3.32$ with periodic boundary conditions in the axial direction. The mean roughness height $\left\langle h_{r}\right\rangle / d=0.02$ is small, so that the effective radius ratio is only slightly affected. We scale the roughness stripe such that the maximum roughness height, and thus the maximum blockage ratio, was $\max \left(h_{r}\right)=0.1 d$. Depending on $\tilde{s}$, we cut out a portion of roughness from the 
scanned surface. The roughness was then mirrored and concatenated to obtain a streamwise homogeneous spanwise periodic stripe.

The sandpaper roughness was implemented in the code by an immersed boundary method (IBM) [52]. In the IBM, the boundary conditions were enforced by adding a body force $\mathbf{f}$ to the Navier-Stokes equations. A regular, non-body fitting, mesh can thus be used, even though the rough boundary has a very complex geometry. We perform interpolation in the spatial direction preferential to the normal surface vector to transfer the boundary conditions to the momentum equations. The IBM has been validated previously $[41,52,53,132]$. A moving average over $10 \times 10$ points is employed to smooth the scan from measurement noise. Finally, we set the resolution based on the demands $\left(\Delta z^{+}, r_{i}^{+} \Delta \theta<3\right)$, which is small enough to recover the smallest geometrical features of the surface.

\subsubsection{Experimental apparatus with spanwise roughness}

The experiments have been performed in the Twente Turbulent Taylor-Couette $\left(\mathrm{T}^{3} \mathrm{C}\right.$ ) facility as shown in figure 4.1a (details of the experimental facility can be found in [80]). The inner cylinder has a radius $r_{i}=200 \mathrm{~mm}$ and the outer cylinder has a radius $r_{o}=279.4 \mathrm{~mm}$, such that the gap size is $d=r_{o}-r_{i}=79.4 \mathrm{~mm}$, and the radius ratio $\eta=r_{i} / r_{o}=0.716$. To minimize the effects of the endplate onto the torque signal, the inner cylinder is partitioned into three sections and the torque is only measured at the middle section $\left(L_{\text {mid }}=536 \mathrm{~mm}\right)$, shown as the highlighted section in figure 4.1a). The combined length of the cylinders is $L=927 \mathrm{~mm}$, which leads to an aspect ratio $\Gamma=L / d=11.7$. The outer cylinder (OC), is made of transparent acrylic which allows for optical access to the flow. The working fluid is demineralised water. We apply spanwise varying roughness to the inner cylinder (IC), which leads to patterns of homogeneously rough and hydrodynamically smooth bands in the spanwise direction (see figure 4.1a). The rough stripes are made of P36 ceramic industrial grade sandpaper and are fixed to the IC using double-sided adhesive tape $(\approx 1 \mathrm{~mm})$. In figure 4.2 , we show the height scan of a roughness element using confocal microscopy. The scan revealed that the height $\left(h_{r}\right)$ of the roughness is mostly within $\pm 2 \sigma\left(h_{r}\right)$ of the mean, giving a characteristic length scale $k \equiv 4 \sigma\left(h_{r}\right)=695 \mu \mathrm{m}$ (see figure $4.2 \mathrm{~b}$ ). More statistics of the roughness is shown in table 4.1. We fix the surface coverage of the roughness at $56 \%$ such that $0.56 A_{i}$ of the cylinder is rough, where $A_{i}=2 \pi r_{i} L$ is the area of the entire IC. The middle section of the inner cylinder has a constant coverage of $56 \%$. 


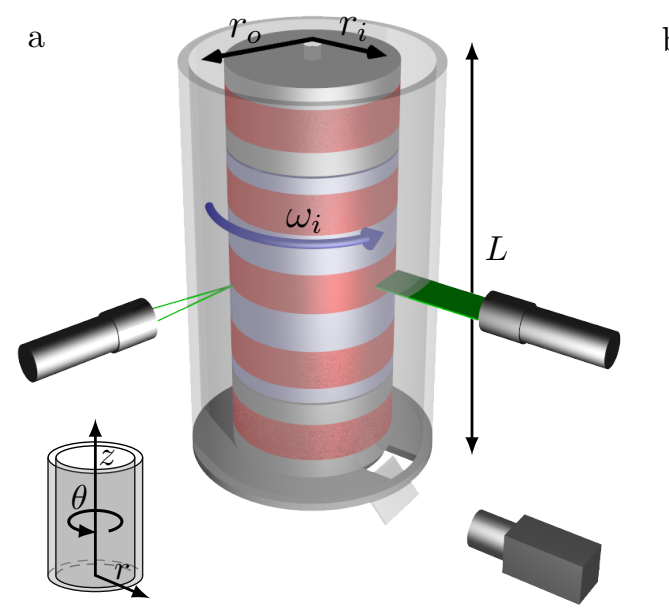

b

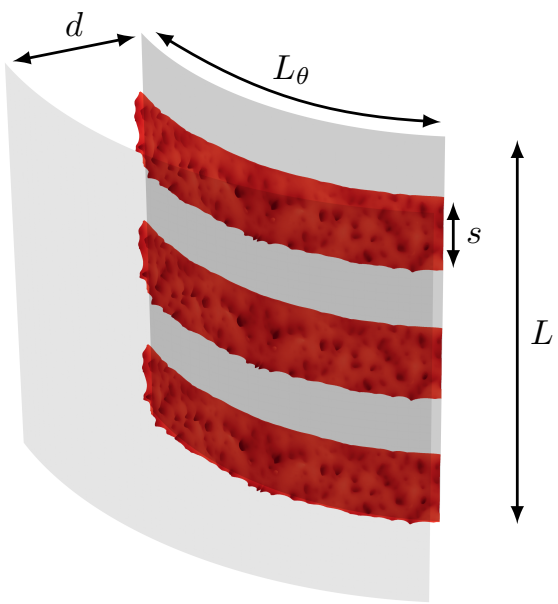

Figure 4.1: a) Schematic of the Twente Turbulent Taylor-Couette facility showing the sandpaper roughness on the inner cylinder in red. PIV measurements in the $r-\theta$ plane become possible thanks to illumination from the side with a high-power pulsed laser, creating a horizontal sheet. The sheet is imaged through a window and a mirror in the bottom of the setup. Using LDA the streamwise velocity is measured along the spanwise direction. The torque is measured in the middle section of the IC (highlighted in blue), which has a length of $\mathrm{L}_{\text {mid }}=536 \mathrm{~mm}$ ( $[100]$ ). b) Numerical domain for the case of $\tilde{s} \equiv s / d=0.47$. The sandpaper roughness is taken from a confocal scan of the material used in the experiment, see figure 4.2. 


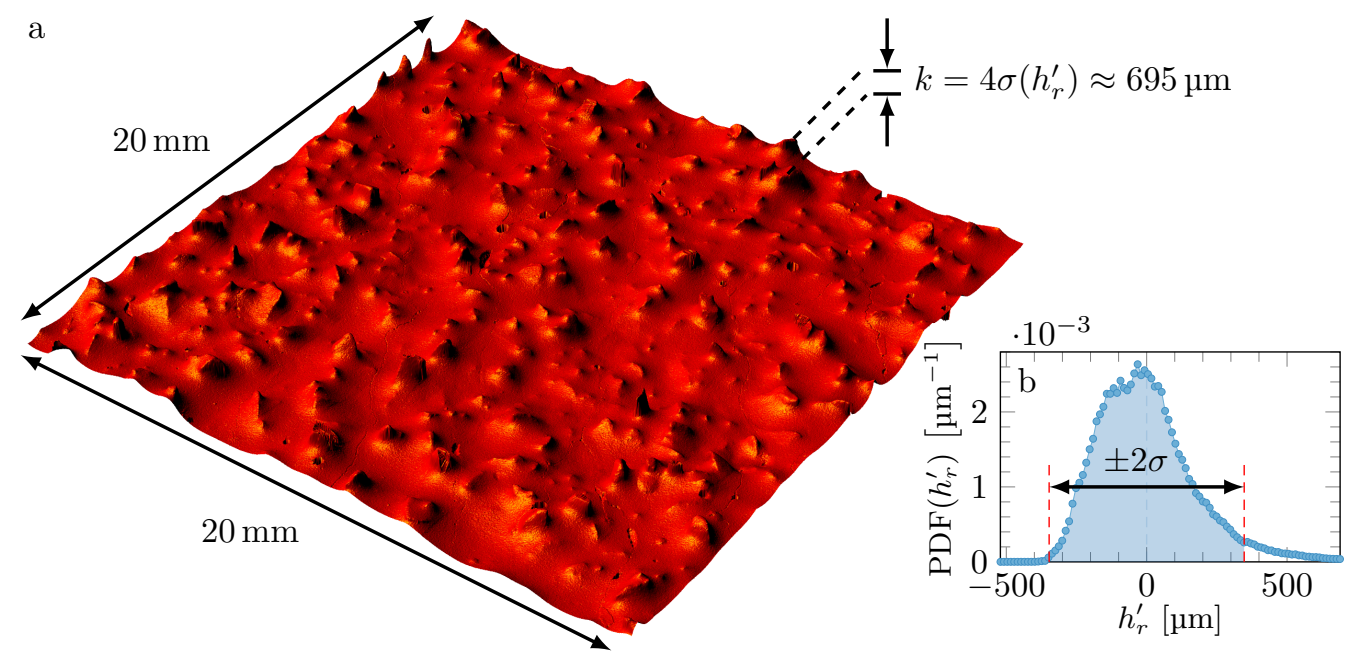

Figure 4.2: a) Height scan captured using confocal microscopy of a patch of sandpaper of $20 \mathrm{~mm} \times 20 \mathrm{~mm}$ with a resolution of $2.5 \mu \mathrm{m}$. The typical size of the grains is given by $k \equiv 4 \sigma\left(h_{r}\right)=695 \mu \mathrm{m}$ where $h_{r}$ is the height and $\sigma$ the standard deviation. The normalized typical grain size is then $k / d \approx 0.01$. b) Probability density function (PDF) of the measured height of the roughness stripe, with subtracted mean $h_{r}^{\prime}=h_{r}-\left\langle h_{r}\right\rangle$. 


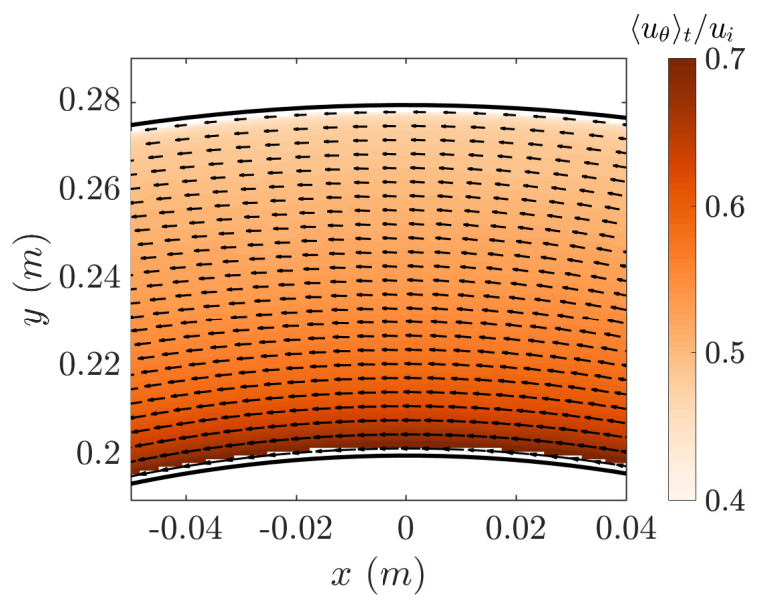

Figure 4.3: Time average of the azimuthal velocity $\left\langle u_{\theta}\right\rangle_{t}$ obtained from PIV measurements for the case of $s / d=1.23$ and $\tilde{z}=0.48$. The resolution of the image field at this height is $9 \mathrm{~cm} / 2560 \mathrm{px} \approx 35 \mu \mathrm{m} / \mathrm{px}$. The color bar and the length of the vectors depicts the value of the azimuthal velocity normalized with the inner velocity $u_{i}=r_{i} \omega_{i}$. For clarity, the vector field is sub sampled roughly $10 \times$. The data that lies within $0.02 d$ away from both the inner and the outer cylinders is omitted as not enough resolution is available to measure the structure of the boundary layers. The black solid lines represent the inner and outer cylinder, respectively. 


$$
\left(\omega-\langle\omega\rangle_{\theta, z}\right) / \omega_{i}
$$

$-0.5$

$-0.25$ 0
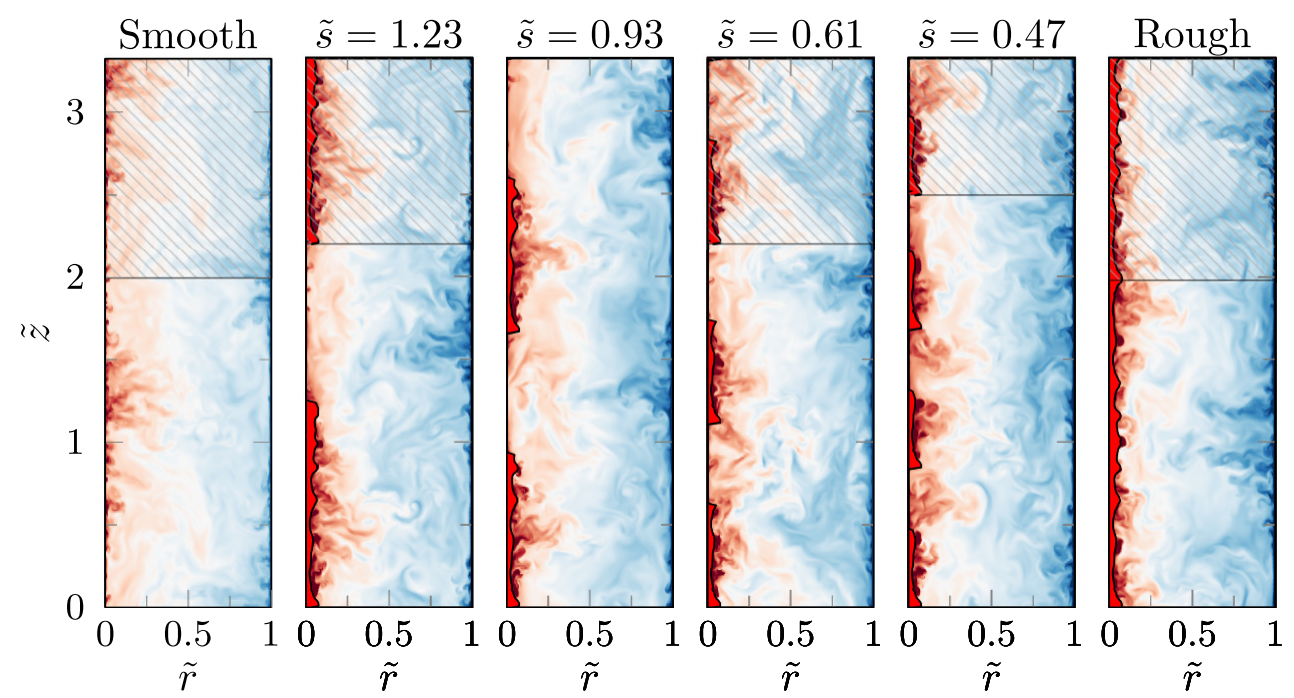

Figure 4.4: Snapshot of the instantaneous angular velocity with the mean angular velocity subtracted, for $\mathrm{Ta} \approx \mathcal{O}\left(10^{9}\right)$. We observe the formation of plumes from the roughness elements whereas very few plumes are formed above the smooth patches. 


\begin{tabular}{cc} 
Metric & Value \\
\hline$\sigma\left(h_{r}\right)=\sqrt{\left\langle h_{r}^{\prime 2}\right\rangle}$ & $174 \mu \mathrm{m}$ \\
$\left\langle\left|h_{r}^{\prime}\right|\right\rangle$ & $134 \mu \mathrm{m}$ \\
$\min \left(h_{r}^{\prime}\right)$ & $-527 \mu \mathrm{m}$ \\
$\max \left(h_{r}^{\prime}\right)$ & $738 \mu \mathrm{m}$ \\
$\operatorname{median}\left(h_{r}^{\prime}\right)$ & $-19.6 \mu \mathrm{m}$ \\
$\operatorname{mode}\left(h_{r}^{\prime}\right)$ & $-27 \mu \mathrm{m}$ \\
$\mathrm{IQR}=\mathrm{CDF}^{-1}(0.75)-\mathrm{CDF}^{-1}(0.25)$ & $215 \mu \mathrm{m}$ \\
$\left\langle h_{r}^{\prime 3}\right\rangle /\left\langle h_{r}^{\prime 2}\right\rangle^{3 / 2}$ & 0.928 \\
$\left\langle h_{r}^{\prime 4}\right\rangle /\left\langle h_{r}^{\prime 2}\right\rangle^{2}$ & 4.361 \\
wetted area $/$ flat area & $\approx 1.6$ \\
$\left\langle h_{r}\right\rangle$ & $1.8 \mathrm{~mm}$
\end{tabular}

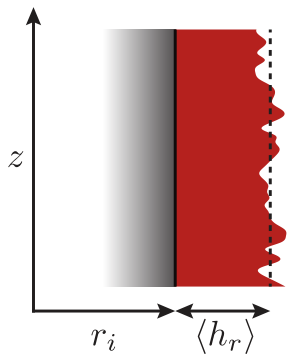

Table 4.1: Various statistics of the roughness $h_{r}^{\prime}=h_{r}-\left\langle h_{r}\right\rangle$ based on the data obtained from confocal microscopy, see also figure $4.2 .\left\langle h_{r}\right\rangle$ is the distance with respect to the smooth cylinder surface. These values represent the actual roughness used in experiments; in DNS a scaled version of these values are used.

\section{Global measurements: Torque}

We measured the torque $\mathcal{T}$ needed to drive the inner cylinder at constant angular velocity (the outer cylinder is kept at rest). For this we used a hollow flange reaction torque transducer connecting the drive shaft and the inner cylinder, sampled at $100 \mathrm{~Hz}$. We continuously measured the torque while quasi-statically ramping the frequency of the inner cylinder, $f_{i}$, from $5 \mathrm{~Hz}$ to $18 \mathrm{~Hz}$, with increments of $0.002 \mathrm{~Hz} \mathrm{~s}^{-1}$. This corresponds to Ta between $4 \times 10^{11}$ and $\mathrm{Ta} \approx 6 \times 10^{12}$. The system is temperature controlled such that all the experiments were performed at $21 \pm 1^{\circ} \mathrm{C}$ and all fluid flow properties are calculated at their actual temperature. Table 4.2 shows additional experimental parameters.

\section{Local measurements: LDA and PIV}

We performed an spanwise scan of the streamwise velocity with laser Doppler anemometry (LDA). The scan was performed at the middle of the gap, $\tilde{r}=$ $\left(r-r_{i}\right) / d=0.5$, at a fixed Ta $=9.5 \times 10^{11}$. Due to the axial symmetry, we scan the flow from mid-height to the bottom of the system. The flow was seeded using $5 \mu \mathrm{m}$-diameter polyamide particles with a density of $1030 \mathrm{~kg} \mathrm{~m}^{-3}$ 
that act as passive tracers [100]. The laser beams went through the outer cylinder and were focused in the middle of the gap. We corrected for curvature effects (and refractive index effects) by numerically ray tracing the LDA beams as was shown in [133]. The spanwise extent of the LDA scans was $0 \leq z / L \leq 0.5$. Particle image velocimetry (PIV) measurements were performed at $\mathrm{Ta}=9.5 \times 10^{11}$ (same as LDA) in the radial-azimuthal plane. The scan was performed for various heights and for all $\tilde{s}$. For the PIV measurements the flow was seeded with different particles that act as passive tracers, namely, fluorescent polymer particles (Dantec FPP-RhB-10) with diameters of $1-20 \mu \mathrm{m}$ with a seeding density of $\approx 0.01$ particles/pixel. These particles have an emission peak with a wavelength of $\approx 565 \mathrm{~nm}$. We illuminated the particles with a Quantel Evergreen $145532 \mathrm{~nm}$, double pulsed laser. A cylindrical lens was used to create a light sheet of $\approx 1 \mathrm{~mm}$ thickness. The height of the laser sheet is altered using a custom traverse system. The images were captured with an Imager SCMOS $(2560 \times 2160$ pixel $) 16$ bit camera with a Carl Zeiss 2.0/100 lens through a window in the bottom plate of the setup and a mirror. The camera was operated in double frame mode with a frame rate $f=15 \mathrm{~Hz}$ which was much smaller than the inverse interframe time $1 / \Delta t$, i.e. $\Delta t \ll 1 / f$. In order to enhance the particle contrast in the images, we added an Edmund High-Performance Longpass $550 \mathrm{~nm}$ filter to the camera lens. For every $\tilde{s}$, the spanwise extent of the experiments was different. This is done because - as will be shown later - the aspect ratio of the rolls change depending on $\tilde{s}$. For the smallest $\tilde{s}=0.63$ however, the spanwise resolution was $\delta z / L \approx 0.011$ while for the largest value $\tilde{s}=3.74, \delta z / L \approx 0.022$. Since we scan in the spanwise direction, the focus of the camera was changed accordingly. For each height, 1000 image pairs were recorded. The fields were resolved with a commercial PIV software (Davis 8.0) based on a multi-step method. The initial interrogation window size was set to $64 \times 64$ pixels and it decreased to $32 \times 32$ pixels for the last iteration, for increased accuracy. The fields are calculated in Cartesian coordinates, which we transformed to polar coordinates. The final result were the fields in the form $\vec{u}=u_{r}(r, \theta, t) \hat{e_{r}}+u_{\theta}(r, \theta, t) \hat{e_{\theta}}$, where $u_{r}$ and $u_{\theta}$ are the radial and streamwise velocity component which depend on the radius $r$, the azimuthal (streamwise) direction $\theta$, and time $t$. For an example of a typical azimuthal velocity field obtained from the experiments, see figure 4.3. 

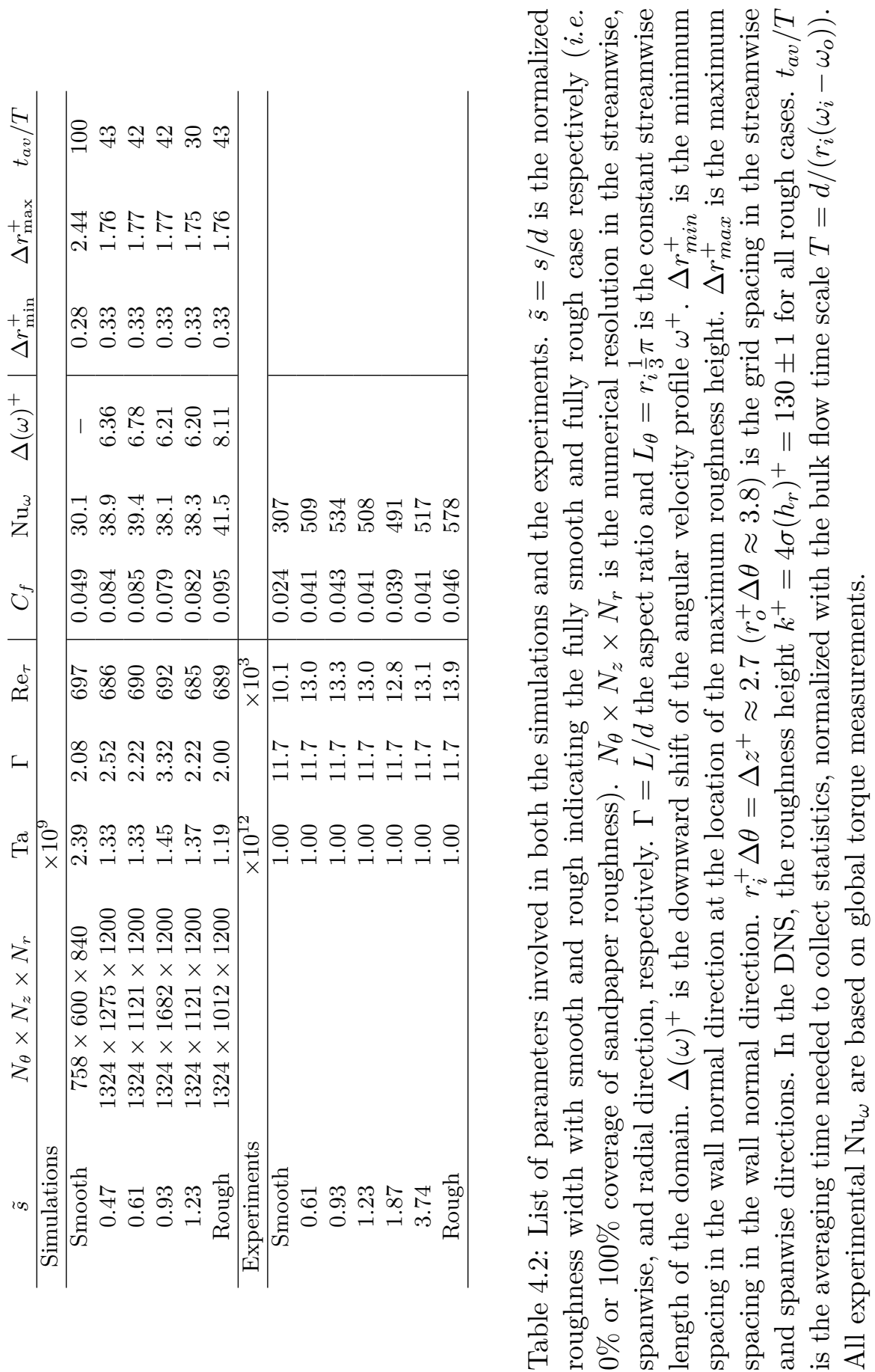


\subsection{Results}

\subsubsection{Response of the Turbulent Taylor Vortices}

\section{Laser Doppler anemometry}

In order to get a first insight on the effect of the roughness on the flow, we performed spanwise scans of the streamwise flow velocity at midgap using LDA. Subsequently, we calculated the standard deviation of the streamwise velocity. In figure 4.5(a), we show the standard deviation of the streamwise velocity $\sigma\left(u_{\theta}\right)$ normalized by the inner cylinder velocity $u_{i}$, as a function of the height, for various $\tilde{s}$. Here, the spanwise coordinate is normalized using the cylinder gap width $d$ such that $\tilde{z}=z / d$. In the top row, we compare the results from direct numerical simulations and experiments. Since we employ different boundary conditions in the spanwise direction DNS (periodic) and experiments (stationary solid plates), the comparison will be most reliable at mid height, where end effects are negligible. Therefore, we overlay the results from DNS (orange lines) over the results from experiments (blue lines) at matching locations with respect to the roughness stripes, at $\tilde{z} \in(1,6)$. In the bottom row we only assess the results from DNS and consequently plot $\tilde{z} \in(0, \Gamma)$.

Figure 4.5(a) reveals that for the case of the largest stripe width $(\tilde{s}=3.74)$, the smooth section has, on average, a value of $\sigma\left(u_{\theta}\right) / u_{i} \approx 0.03$, slightly larger than we found for the fully smooth case (shown with the dotted light blue lines).

Above the rough stripe, towards the center of the setup (i.e. for large $\tilde{z}$ ), $\sigma\left(u_{\theta}\right)$ gradually increases to a value of approximately $\sigma\left(u_{\theta}\right) / u_{i} \approx 0.04$. A similar, but not so clear trend can be seen at the lower roughness section $(\tilde{z} \approx 0.93)$ of this case. However, this might be influenced by the lower bottom plate of the system.

When looking at the $\tilde{s}=1.87$ case, we see very similar, however more pronounced dynamics. Streamwise velocity fluctuations are promoted in regions where the roughness is present, as suggested by the appearance of local peaks centered at the position of the rough stripes. This effect is further seen for the cases of $\tilde{s}=1.23$ and $\tilde{s}=0.93$, where we observe similar profiles. At their smooth areas however, we also observe enhanced velocity fluctuations, albeit less pronounced than the locations above the rough patches. This effect is not seen for $\tilde{s}>1.27$. For the final case with $\tilde{s}=0.61$ these trends seems to fade away and we see that $\sigma\left(u_{\theta}\right)$ becomes more spanwise independent, i.e. 

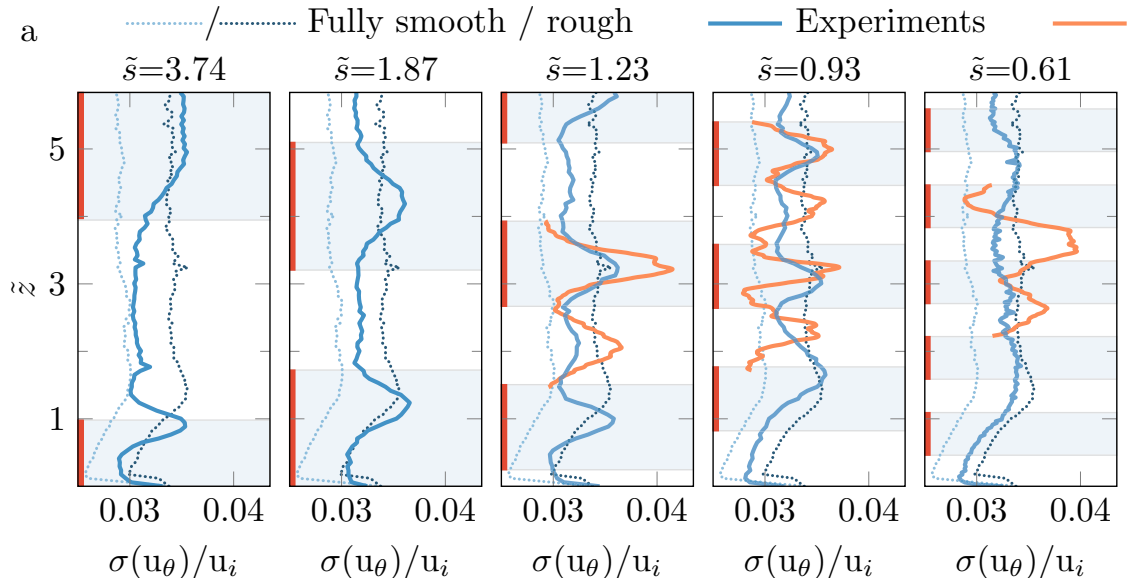

Simulations

$$
\sigma\left(\mathrm{u}_{\theta}\right) / \mathrm{u}_{i}
$$$$
\sigma\left(\mathrm{u}_{\theta}\right) / \mathrm{u}_{i}
$$
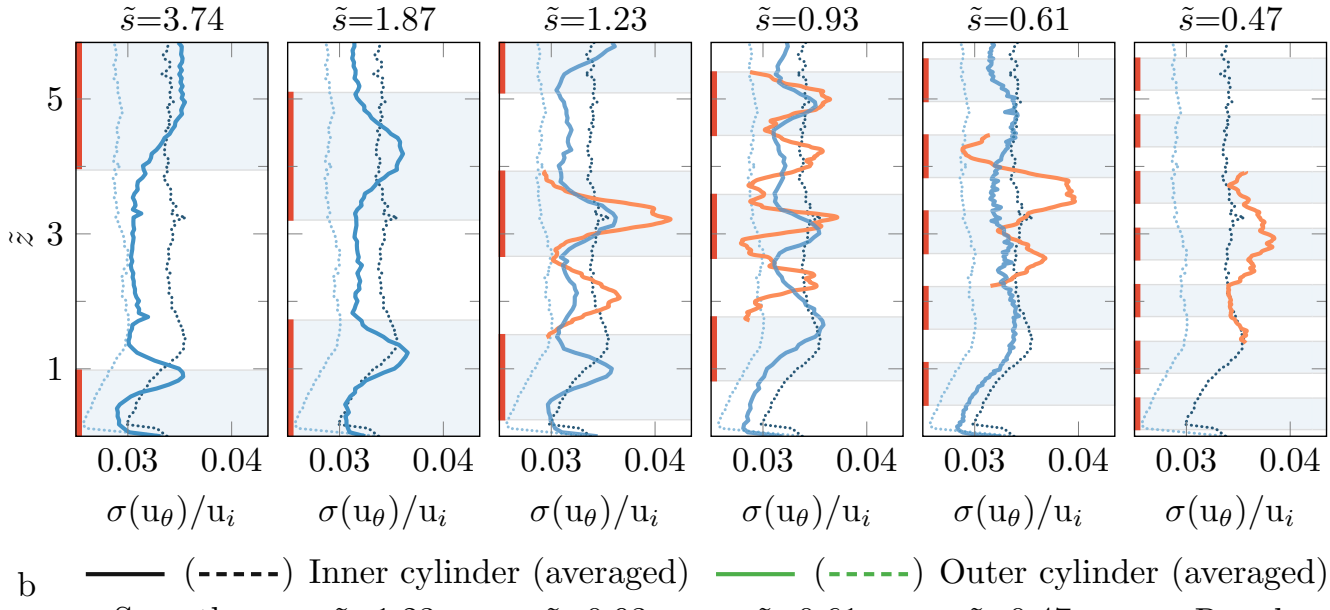

(-----) Outer cylinder (averaged)
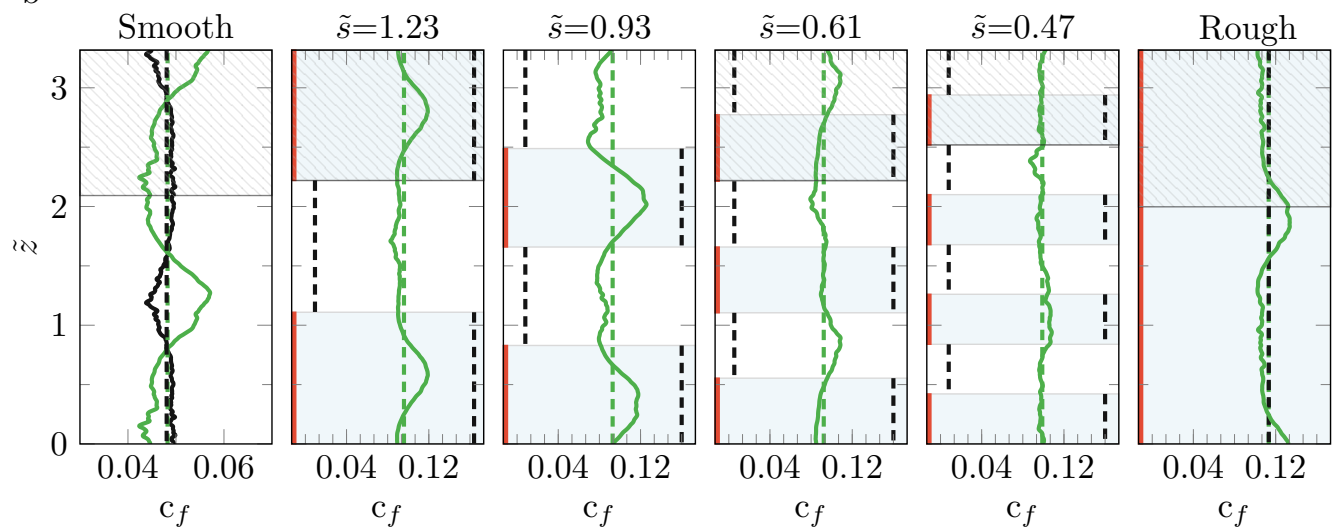

Figure 4.5: (a) Normalized standard deviation of the streamwise velocity $\sigma\left(u_{\theta}\right) / u_{i}$ at mid gap, as a function of $\tilde{z}=z / d$ for various $\tilde{s}$. Experimental data is captured using LDA while Ta is fixed at $1 \times 10^{12}$. For DNS, Ta is set to $\mathcal{O}\left(10^{9}\right)$ for all cases. The enforced roughness pattern is indicated in red and a light blue shade. The signature of the roughness pattern is clearly visible in the bulk flow. For $\tilde{s}=0.61$, the roughness pattern does not leave a distinct imprint of its topology in the midgap flow statistics. Fully smooth and fully rough reference cases from experiments are shown as dotted lines. (b) Local friction factor $c_{f}(\tilde{z})$ versus the axial height $\tilde{z}=z / d$ for Ta $=\mathcal{O}\left(10^{9}\right)$, based on DNS data. The black lines show $c_{f}(\tilde{z})$ at the IC and the green line show $c_{f}(\tilde{z})$ at the OC. $c_{f, r}$ above the rough patches was calculated by subtracting the smooth average of $c_{f, s}$ from $C_{f}=\left\langle c_{f}(\tilde{z})\right\rangle_{L}$ of the entire IC. Hatched regions indicate spanwise translated copies of the same data (including averages) possible due to the periodic boundary condition in the axial direction. 
the peaks are less pronounced, and do not seem to follow the topology of the roughness stripes.

The results of the DNSs, presented together with the experiments in figure 4.5(a) exhibit very similar behaviour. When normalized with $u_{i}$, we find that the standard deviation of the streamwise velocity show similar values as in the experiments. This is intriguing since the $k / d$ values in the simulations are almost one order of magnitude larger than in the experiments. Above the rough stripes, we find enhanced $\sigma\left(u_{\theta}\right) / u_{i}$, whereas over smooth stripes we find diminished $\sigma\left(u_{\theta}\right) / u_{i}$. For $\tilde{s}=0.61$ however, the trends are somewhat different. There, we find enhanced $\sigma\left(u_{\theta}\right) / u_{i}$ above the smooth region in the DNSs, which is explained by the recombination of plume ejection regions, forming one larger TTV above the smooth regions, see figure 4.7.

The findings presented in figure 4.5(a) show that the presence of the roughness affects the relative turbulence statistics in the bulk of the flow, far away from the roughness sublayer region (in contrast to homogeneous roughness in TC flow [96]), reminiscent to what is found in studies of pipe and channel flow $[114,116]$.

Figure $4.5(\mathrm{~b})$ shows the spanwise variations of the friction factor $c_{f}(\tilde{z})$ (see section 4.1) on both the inner cylinder and the outer cylinder. The solid and dashed black lines represent $c_{f}(\tilde{z})$ measured on the inner cylinder, and the solid (green) lines represent $c_{f}(\tilde{z})$ on the outer cylinder. We average both in time and in the streamwise direction. Significant variations in $c_{f}(\tilde{z})$ are observed which are linked to the orientation of the TTV. For impacting regions, i.e. plumes impacting on the inner cylinder boundary layer, the wall shear stress in the streamwise direction is enhanced. When plumes eject from the inner cylinder boundary layer, also known as the ejecting regions, the shear stress is reduced. This, once more, illustrates the relative strength of the secondary flow (TTV) and the mean flow. For $\tilde{s}=0.93$, the variations of the friction factor on the outer cylinder are even more pronounced, thus indicating that the strength of the TTV are enhanced with enforcing the spanwise variations in the roughness. For $\tilde{s}=0.47$, the variations are not visible and the TTV are severely weakened, but still present, see figure 4.7 .

\section{Particle imaging velocimetry and direct numerical simulations}

To gain more insight into how the roughness alters the flow, we set out to measure the velocity field in the meridional plane using PIV at multiple heights. For the cases that required the highest axial resolution $(\tilde{s}=0.61$ and $\tilde{s}=0.93)$ of the PIV measurements we employed $\delta_{z} / d \approx 0.13$, where $\delta_{z}$ is the spacing 


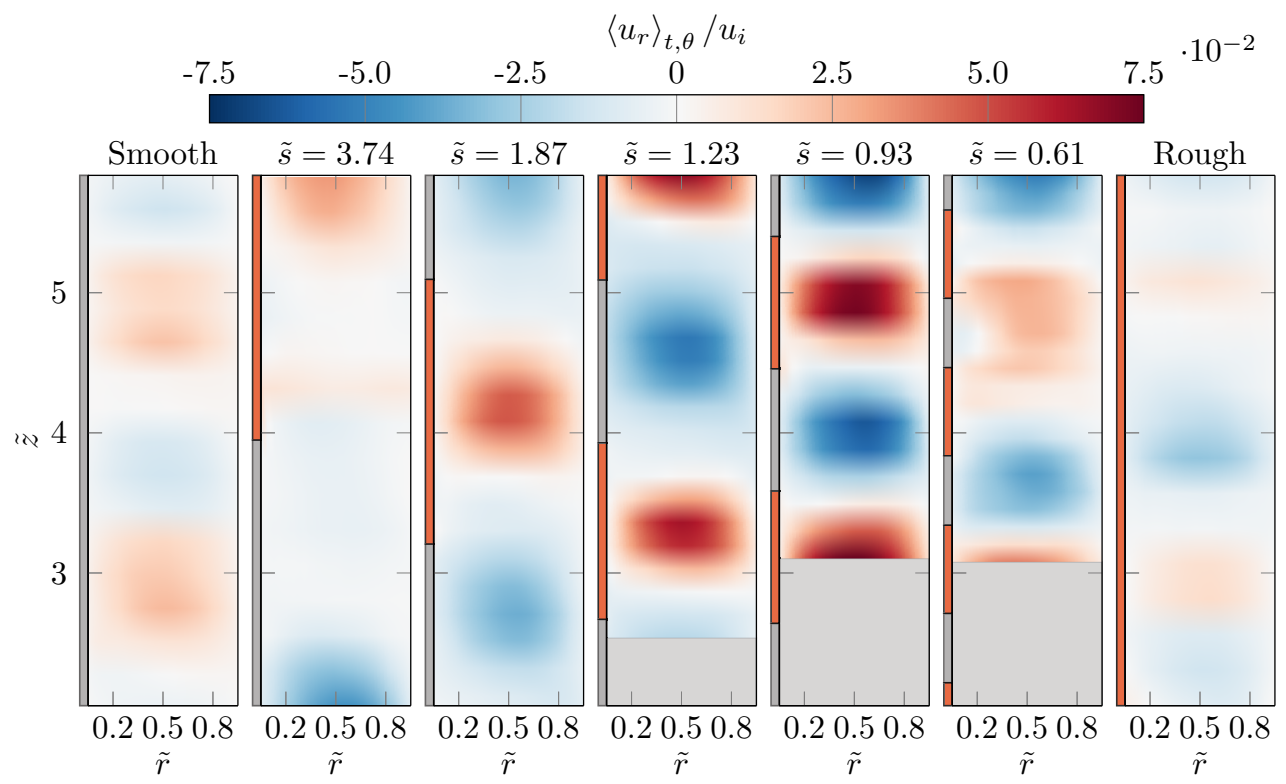

Figure 4.6: Temporal and streamwise average of the radial velocity $u_{r}$, normalized with the inner cylinder streamwise velocity $u_{i}$, obtained from experiments at $\mathrm{Ta}=1 \times 10^{12}$ using PIV for varying roughness stripe sizes $\tilde{s}$. A positive value of $u_{r}$ denotes outflow, while a negative value denotes inflow, with respect to the inner cylinder. It can be seen that the rolls are pinned by the roughness and their wavelength changes with $\tilde{s}$. The red and gray areas at the left side of each plot indicate the positions of the rough and smooth areas, respectively. Note that the typical grain size is $k / d \approx 0.01$. The gray shaded areas in the gap represent unexplored heights. 
of laser sheets in $z$, so that every TTV roll pair was at least sampled 7 times. This is well enough to resolve the wavelength of the structure. For all other $\tilde{s}$, the sample resolution is higher than 7 times per wavelength. In figure 4.6, we show the temporal and streamwise averaged radial velocity component $u_{r}$, normalized with $u_{i}$, in the spanwise wall-normal plane $(\tilde{z}-\tilde{r})$, where the radial coordinate is normalized such that $\tilde{r}=\left(r-r_{i}\right) / d$. Figure 4.6 shows that for the case of $\tilde{s}=3.74$, a very large structure can be seen, which consists of a large outflow region (positive $u_{r}$ ) around $\tilde{z}=5.84$, while a large inflow region (negative $u_{r}$ ) is detected around $\tilde{z}=2.10$. The situation is more pronounced for the cases of $\tilde{s}=1.87, \tilde{s}=1.23$, and $\tilde{s}=0.93$, where a clear roll-like structure (i.e. the TTV) can be observed. Note that the radial component in the flow changes sign along the spanwise direction as it should in the presence of a TTV. What is rather remarkable is that the wavelength of the rolls $\lambda$ changes for different values of $\tilde{s}$. For the large structure at $\tilde{s}=3.74$, the normalized wavelength is $\tilde{\lambda}=\lambda / d \approx 4.01$. As $\tilde{s}$ decreases to $\tilde{s}=1.87, \tilde{\lambda} \approx 1.49$. At $\tilde{s}=1.23$, the wavelength decreases to a value of $\tilde{\lambda} \approx 1.42$. At $\tilde{s}=0.93$, $\tilde{\lambda} \approx 0.94$, and finally for the smallest value of $\tilde{s}=0.61$, the wavelength increases slightly to $\tilde{\lambda}=1.09$. We remind the reader the work of [101], who revealed that for counter-rotation $\left(-\omega_{0} / \omega_{i} \approx 0.4\right)$, the average wavelength of the rolls could be either $\tilde{\lambda}=1.46$ or $\tilde{\lambda}=1.96$ depending on the state attained by the system. The current work shows that by an appropriate choice of $\tilde{s}$, the wavelength of the rolls can firstly, abandon its natural state; and secondly, it can be tuned within the range $\tilde{\lambda} \in[0.94,4.01]$. The wavelengths described above were calculated by measuring the locations of two consecutive maxima and minima of $\left\langle u_{r}\right\rangle_{t, \theta, r_{b u l k}}$ along $z$ which are closest to midheight. Here, the symbol $\langle\cdot\rangle_{t, \theta, r_{b u l k}}$ denotes average over time, the streamwise direction and the bulk region, i.e. $\left(r_{b u l k}-r_{i}\right) / d \in[0.3,0.7]$.

In addition, we observe that outflow regions (flow away from the IC) are created in spanwise regions where the roughness is located; and conversely, inflow regions (flow towards the IC) are created in the smooth areas. Note that this orientation of the secondary flows is opposite to what is found in other canonical systems (e.g. pipe flow and channel flow $[116,125,134]$ ), where one finds inflow regions above the rough stripes and outflow region above the smooth stripes. Consistent with findings in boundary layers however, is the correlation between low momentum pathways (LMPs) and outflow regions [124]. Indeed we find that LMPs - with respect to the IC and located on top of the roughness - is associated with outflow regions, similar to [124] although their Lego ${ }^{\circledR}$ roughness strips are much thinner and protrudes farther into the 
flow.

Another interesting observation is that since the driving is now from the $\mathrm{BL}$ rather than the bulk, the strength of the rolls changes depending on the value of $\tilde{s}$, as evidenced by the magnitude of $\left|u_{r}\right|$. In order to explore this feature in more detail, we quantify the strength of the rolls by ${\tilde{u_{r}}}^{\prime} \equiv \sqrt{\left\langle\left(u_{r} / u_{i}\right)^{2}\right\rangle_{t, \theta, r_{b u l k}, z_{\lambda}}}$ as a function of $\tilde{s}$. Here, the symbol $\langle\cdot\rangle_{t, \theta, r_{b u l k}, z_{\lambda}}$ denotes an average over time, the streamwise direction, the bulk region, and the spanwise region that defines the wavelength of a single roll $z_{\lambda}$. In figure $4.8(\mathrm{c})$, we show ${\tilde{u_{r}}}^{\prime}$ as a function of $\tilde{s}$, where we observe that the strength of the rolls increases with decreasing $\tilde{s}$ for $\tilde{s} \in[0.93,3.74]$. However at $\tilde{s}=0.61$ the trend is broken, and we observe that $\tilde{u_{r}}{ }^{\prime}$ decreases with respect to the case of $\tilde{s}=0.93$.

In order to obtain more insight into the mechanism(s) that lead to the variation of $\tilde{\lambda}$ with $\tilde{s}$, we turn to DNS, albeit at a much lower Ta $\left(\approx 1.0 \times 10^{9}\right)$, and much higher roughness height $(k / d \approx 0.1)$. DNS at this high Re are very computationally expensive and we choose to simulate $\Gamma \geq 2$ for which [46] showed that, at least for smooth walls, the aspect ratio is sufficient. Since very large $\tilde{s}$ cases are not feasible for DNS, we focus on matching the exact $\tilde{s}$ in the lower range. We will show that, despite the $\mathcal{O}\left(10^{3}\right)$ difference in Ta, the same observations found in the numerics are also found in the experiments.

First, we look at the streamwise velocity component. In figure 4.7, we plot the difference of the temporal and streamwise average of the angular velocity $\langle\omega\rangle_{t, \theta}$ with respect to the temporal, streamwise and, spanwise average of the angular velocity $\langle\omega\rangle_{t, \theta, z}$. This is done to emphasize the underlying organization of the TTVs. Here, we clearly observe that for all $\tilde{s}$, ejecting regions of angular velocity are originated in the rough stripes, similar to the preferential plume ejection sides at the tips of grooves in [41]. These ejecting regions advect fluid from the roughness stripe on or at the inner cylinder towards the outer cylinder. These ejecting regions occur at each roughness stripe and as a consequence, an array of plume-like structures are formed along the spanwise direction. In TC flow (without roughness), plume-like structures are clear signatures of the presence of TTVs [45]. A closer inspection of figure 4.7 reveals that for the largest value of $\tilde{s}=1.23$, the plumes have enough separation not to interact among them. When $\tilde{s}$ is decreased to $\tilde{s}=0.93$, we observe that the plumes come closer, and can, in fact, interact with each other. At the lower $\tilde{s}=0.61$ however, the situation is rather different.

The rough patches are too close to each other to create individual ejecting regions and therefore, merge to a larger collective plume. For the $\tilde{s}=0.61$ case, we observe in both, experiments and DNS, that two ejecting regions, each 


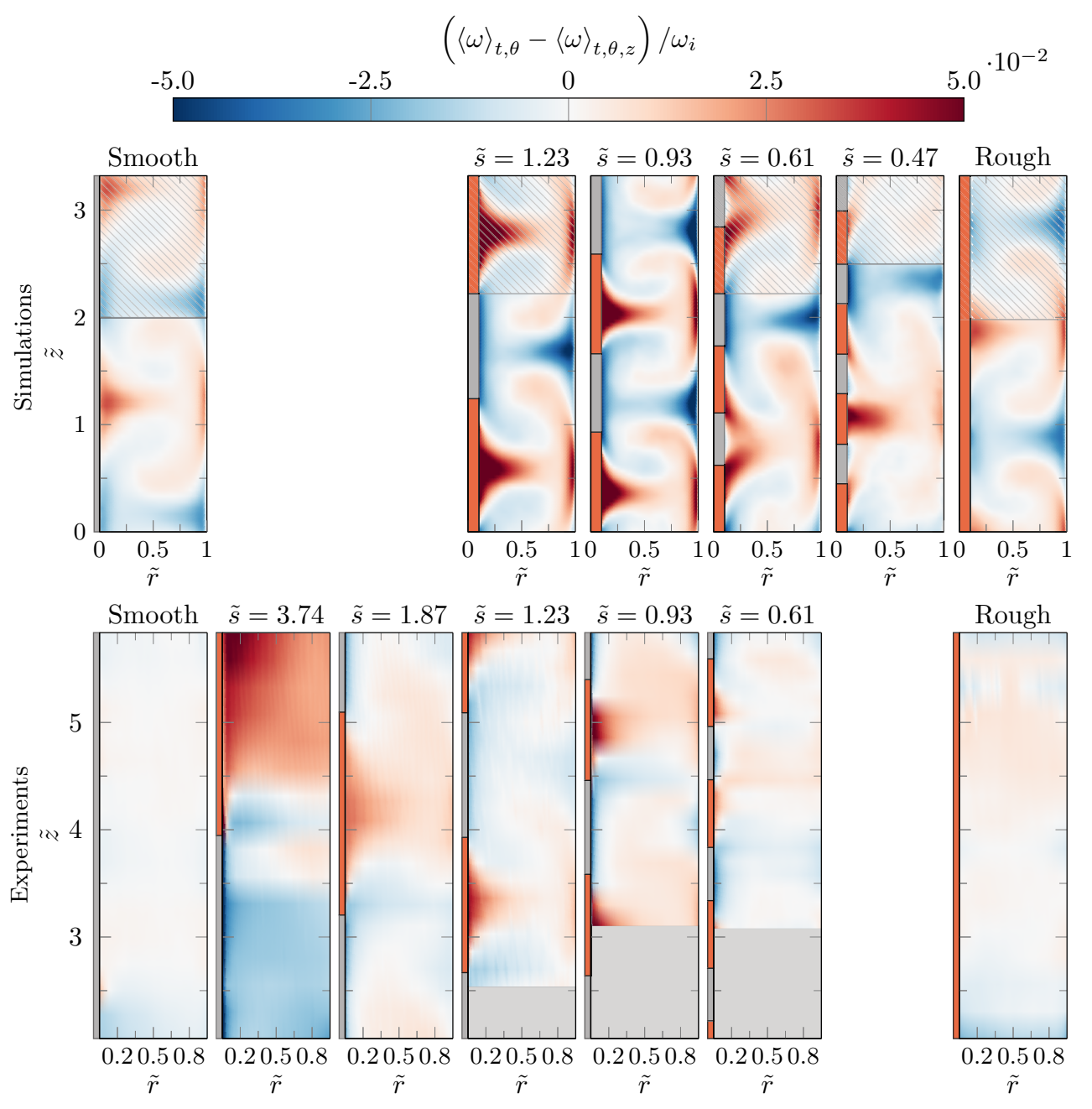

Figure 4.7: Deviation of the temporal and streamwise averaged angular velocity $\langle\omega\rangle_{t, \theta}$ with respect to the temporal, streamwise, and spanwise averaged angular velocity $\langle\omega\rangle_{t, \theta, z}$ obtained from DNS at Ta $\approx \mathcal{O}\left(10^{9}\right)$ (top), and experiments at $\mathrm{Ta}=1 \times 10^{12}$ (bottom), for various $\tilde{s}$ explored. For experiments, $\tilde{r}$ spans between $[0.05,0.95]$. All fields are normalized with the angular velocity of the inner cylinder $\omega_{i}=u_{i} / r_{i}$. Positive values represent velocities that are closer to the IC velocity. The leftmost panel corresponds to the case of no roughness (smooth) while the rightmost panel is the case where the entire IC is uniformly rough. For better comparison, overlapping $\tilde{s}$ cases for DNS and experiments are aligned vertically. Missing cases are not feasible in experiments or DNS. 
located on top of a rough region, merge into a combined ejecting region. When $\tilde{s}$ is decreased to a value of 0.47 , even three ejecting regions are combined to a single large plume. These combined plumes drive the TTV with a larger wavelength as compared to the $\tilde{s}=0.93$ case, and therefore, decreasing the effective momentum transfer.

The mechanisms leading to this optimum in transport remain elusive. We hypothesize that very low $\tilde{\lambda}$ constraints the roll structure too much, and ejecting and impacting plumes hinder each other, limiting the amount of transport. For high $\tilde{\lambda}$, the density of ejecting and impacting zones is too low, and the amount of transport is also limited.

The LDA, PIV and DNS explored in this section reveal that there is a mean effect of the spanwise-varying roughness on the large scale secondary flows that exist in turbulent TC flow. We have seen thus far that the roughness pins the rolls, and that their wavelength and strength can be tuned depending of the choice of $\tilde{s}$ for a variety of $\mathrm{Ta}$, and for a variety of roughness heights $h$. However, how does the flow respond globally, i.e. the angular momentum transport, to this change in morphology? This will be addressed in the following section.

\subsubsection{Global response}

We have performed torque measurements at an acquisition rate of $100 \mathrm{~Hz}$ at identical conditions, only varying the acceleration (ramping time). All measurements accelerated from $0 \mathrm{~Hz}$ to $20 \mathrm{~Hz}$ at run times that varied between 30 minutes and 10 hours. The measured torque signal was binned and compared between various runs. If the difference between the curves is smaller than the measurement error of the sensor $(0.25 \%)$, we call the runs indistinguishable. This is already the case for runs of 60 minutes.

The global response of the TC system can be expressed with $\mathrm{Nu}_{\omega}$ (equation 4.2) or with the related friction coefficient $C_{f}$. In figure 4.8(a), we show the compensated $\mathrm{Nu}_{\omega}$ as a function of the driving strength Ta, where a scaling of $\mathrm{Nu}_{\omega} \propto \mathrm{Ta}^{\alpha}$, with $\alpha=0.45$ is observed for all the $\tilde{s}$ explored. The shaded area indicates the error based on the standard deviation from three repeated measurements. The same data is represented as $C_{f}$ versus $\operatorname{Re}_{i}$ in figure 4.8(d). In this figure, we also give best fits of the Prandtl friction law, defined as $1 / \sqrt{C_{f}}=a \log _{10}\left(\operatorname{Re}_{i} \sqrt{C_{f}}\right)+b$, for the fully smooth and fully rough cases. In absence of roughness and within the same range of Ta, the scaling is found to be effectively $\mathrm{Nu}_{\omega} \propto \mathrm{Ta}^{0.39}[47,49,94,101,135]$. In contrast, when both of the solid walls are made uniformly rough (i.e. pressure drag domi- 

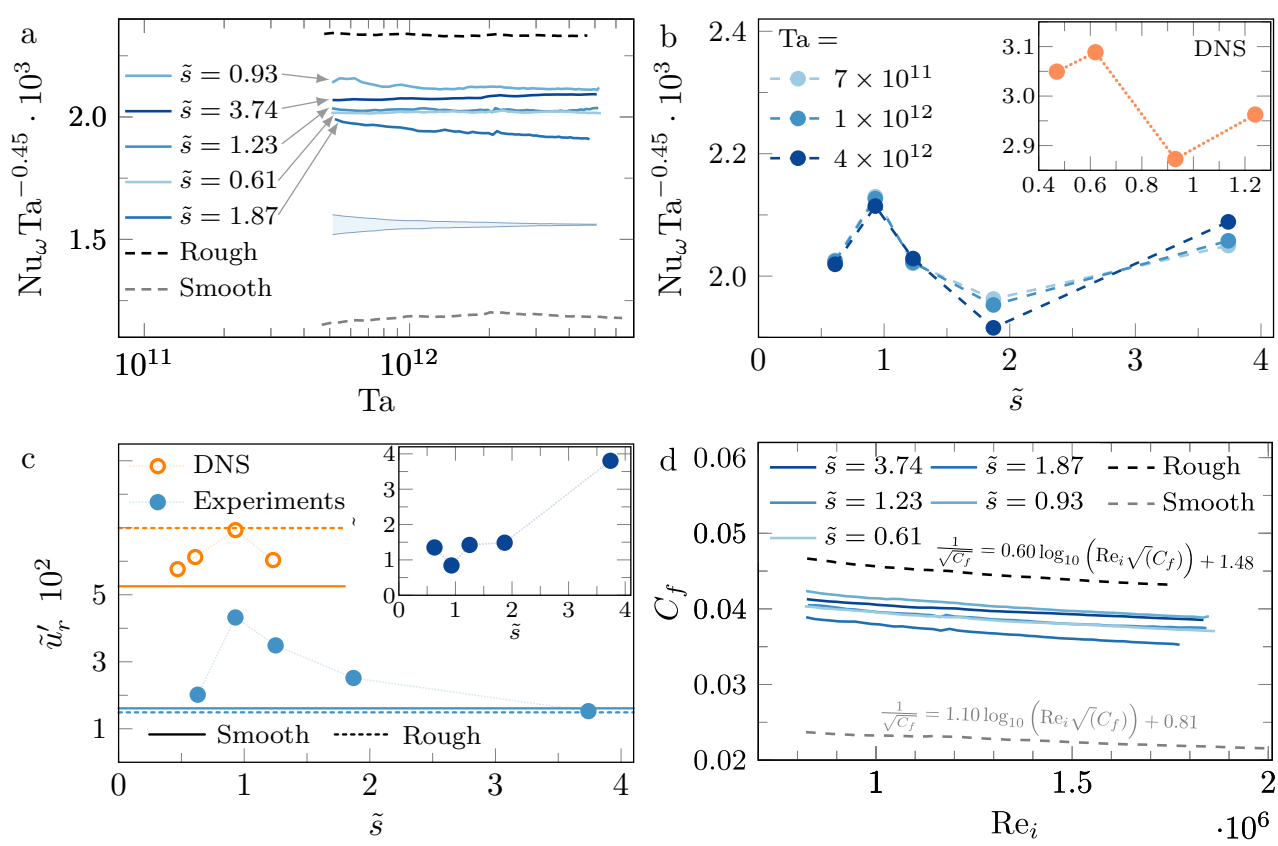

Figure 4.8: (a) Compensated global Nusselt number $\mathrm{Nu}_{\omega} \mathrm{Ta}^{-0.45}$ as function of Ta for varying $\tilde{s}$, based on torque measurements. The shaded area indicates the error based on the standard deviation from repeated measurements, which can be seen to decrease with increasing driving strength. (b) Compensated global Nusselt number $\mathrm{Nu}_{\omega} \mathrm{Ta}^{-0.45}$ as a function of $\tilde{s}$ for three selected $\mathrm{Ta}$, again calculated from torque measurements. Here, an optimum value in the transport of angular momentum is observed close to $\tilde{s} \approx 1$. We note that the optimum could in principle be located anywhere between $0.61<\tilde{s}<$ 1.23. More experiments in this domain are needed to pinpoint the location of the maximum. The inset in (b) shows the results obtained with the DNS $\left(\mathrm{Ta} \approx 10^{9}\right)$, where the maximum can be observed at a slightly lower $\tilde{s}$, namely $\tilde{s} \approx 0.6$. (c) The strength of the rolls, quantified as the normalized RMS of the radial velocity $\tilde{u}_{r}^{\prime}$ as a function of $\tilde{s}$, for both, DNS and experiments. Solid and dashed lines show fully smooth and rough respectively. The inset shows the wavelength of the TTV as function of $\tilde{s}$. (d) Global friction coefficient $C_{f}$ as a function of the driving strength, expressed with the Reynolds number $\operatorname{Re}_{i}$, for various $\tilde{s}$, based on the torque data. The order of the curves is identical to figure 6a. For fully smooth and fully rough, the best fit of the Prandtl friction law are shown. 
nates), the scaling asymptotes to the ultimate regime predicted by Kraichnan, i.e. $\mathrm{Nu}_{\omega} \propto \mathrm{Ta}^{0.5}[43,72]$. In [43], the closest configuration to our study is the case of rough IC and smooth OC, for which an effective exponent $\alpha=0.43$ was found. We note that this exponent is slightly smaller than the ones observed in the current study. The reason behind this is currently unknown. We notice, however, that the roughness type in our study is rather different. In this study we use spanwise-varying sand grain roughness, while the roughness in [43] is made of rib obstacles and is oriented perpendicular to the streamwise direction.

In order to connect the observed dynamics of the TTVs with the global response, we plot in figure 4.8(b) the compensated Nusselt number $\mathrm{Nu}_{\omega} \mathrm{Ta}^{-0.45}$ as a function of $\tilde{s}$ for both the experiments and the numerics. We note that the exponent found for $\tilde{s}=1.87(\alpha=0.44)$ is slightly smaller than $\alpha=0.45$. We notice rather remarkably, the appearance of a maximum around $\tilde{s} \approx 0.93$ for the experiments, and $\tilde{s}=0.61$ for the DNS (shown in the inset of figure $4.8(\mathrm{~b}))$. We attribute the appearance of this peak to the strengthening of the TTVs, which is caused by the variation of $\tilde{s}$, and thus of $\tilde{\lambda}$. Explicitly, by lowering $\tilde{s}$, we can decrease the wavelength of the rolls, as seen in the inset of figure 4.8(c), thereby, bringing them closer together (see also section 4.3.1). As a consequence, the rolls are strengthened which leads to an enhancement of the angular momentum transport; and thus, the peak around $\tilde{s}=0.93$. This peak is also visible in the normalized RMS of the radial velocity, $\tilde{u}_{r}^{\prime}$, plotted in figure $4.8(\mathrm{c})$. With decreasing $\tilde{s}, \tilde{u}_{r}^{\prime}$ increases until an optimum is been reached around $\tilde{s}=0.93$. Below the optimum, $\tilde{u}_{r}^{\prime}$ drops drastically to much lower values. The PIV measurements are carried out over a domain $0.3<\left(r-r_{i}\right) / d<0.7$, so that the value of $\tilde{u}_{r}^{\prime}$ in the boundary layers is not included. However, the DNSs give us the value of $\tilde{u}_{r}^{\prime}$ throughout the entire domain, such that we include the values in the boundary layers. Considering that $\tilde{u}_{r}^{\prime}$ peaks in the BL, we can understand the higher values of $\tilde{u}_{r}^{\prime}$ for the DNS. Regardless of this, we find similar behavior between the DNS and the experiments.

The optimum in transport is also observed by [101], though the mechanism leading to optimum transport there is quite different. While the rolls in their study are enhanced by counter-rotating the OC; in our case, the rolls are strengthened by forcing $\tilde{\lambda}$ below their natural wavelength due to the right choice of the size of the spanwise varying roughness $\tilde{s}$. This is also supported by the observation that the magnitude of the radial velocity shows a maximum around $\tilde{s}=0.93$, as shown in figure $4.8(\mathrm{c})$. We note, however, that the torque 
is not measured throughout the entire spanwise length of the cylinders $L=$ $927 \mathrm{~mm}$, but in a smaller section of length $L_{\text {mid }}=536 \mathrm{~mm}$. As a result, the large structure identified previously for the case of $\tilde{s}=3.74(\tilde{\lambda}=4.01)$, does not fit entirely in the measurement section (see the first panel of figure 4.6). the measured torque will be higher if part of the ejecting region (with respect to the IC) is excluded from the measurement zone, since there we experience a lower $c_{f}$, see figure $4.5(\mathrm{~b})$. The measured torque will consequently be lower if part of the impacting region (with respect to the IC) is excluded from the measurement zone, since there we experience a higher $c_{f}$, see figure $4.5(\mathrm{~b})$. We also note that in the case of the numerics, the position of the maximum is different than in the experiments. We attribute this to a combination of two effects. On the one hand the DNS is performed at a lower Ta, which has an effect on the natural wavelength of the rolls as it was shown by [128], who show that for similar values of $\eta$, the wavelength of the rolls can decrease with decreasing Ta. On the other hand, the spanwise domain of the DNS is bounded by $\Gamma \in[2.08,3.32]$, which gives rise to limited box-sizes. Thus, when $\tilde{s}$ is varied, the rolls could suffer from an additional constraint due to the limited spanwise domain. In addition to this discrepancy, we also note that the scaling in the range of Ta at which the DNS is done $\left(\approx 1.0 \times 10^{9}\right)$, is not known a priori. In absence of a better choice, we compensate the numerical data using the same exponent as in the experiments (figure 4.8(b)). However, we note that this exponent might be different due to the 2 decades of separation in Ta between the numerics and experiments, as was also shown by [43]. We would like to emphasize, however, that in spite of these discrepancies, a maximum in angular momentum transport is observed for a given $\tilde{s}$ in both the experiments and the numerics, which is solely a consequence of the varying spanwise wavelength of the TTV, dictated by the spanwise-varying roughness.

\subsubsection{Velocity profiles}

Having discussed the dynamics of the TTVs and the corresponding global response, in terms of the dimensionless torque, we now set out to study the streamwise, angular, velocity profiles (rather than the azimuthal profiles, as discussed in [77] and [96]). To allow for straightforward comparison between the respective velocity profiles, we run the DNS at constant friction Reynolds number $R e_{\tau}=690 \pm 10$. The profiles are then temporally, streamwise, and spanwise averaged $\omega^{+}=\langle\omega\rangle_{t, \theta, z} / \omega_{\tau}$. The profiles still exhibit a logarithmic region when averaged over the entire spanwise coordinate. Figure 4.7 shows however that the TTVs in the flow, following the spanwise-varying roughness, 

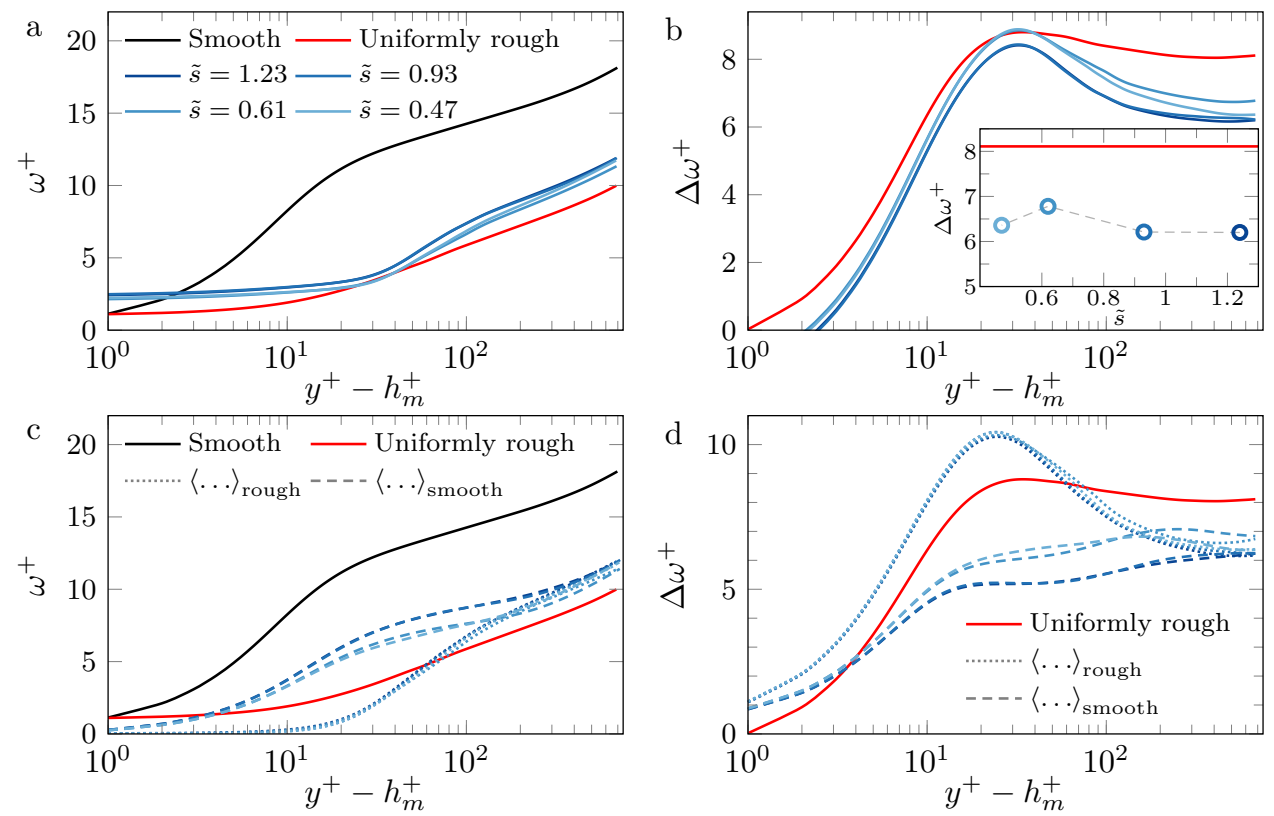

Figure 4.9: Angular velocity profiles and angular velocity shifts based on DNS. (a) Angular velocity $\omega^{+}$profiles in the reference frame of the IC versus the wall normal distance $y^{+}-h_{m}^{+}$for various $\tilde{s}$, where $h_{m}^{+}$is the virtual origin and equals the melt-down (i.e. mean) height $h_{m} / k$ of the rough surface and $y^{+}=\left(r-r_{i}\right) / \delta_{\nu}$. The solid black line represents the uniformly rough case. (b) Angular velocity shift $\Delta \omega^{+}$as a function of $y^{+}-h_{m}^{+}$for varying $\tilde{s}$. In the inset of (b), we show the angular velocity shift $\Delta \omega^{+}$versus the wall normal distance $y^{+}-h_{m}^{+}$. Here, we observe a maximum downward shift $\left(\Delta \omega^{+}=\omega_{s}^{+}-\omega_{r}^{+}>0\right.$, where subscripts $s$ and $r$ represent the smooth and rough profiles respectively) of the angular velocity profile for the simulation where we cover the entire inner cylinder with sandpaper roughness (i.e. uniformly rough). (c) The angular velocity conditioned on the spanwise location: above smooth surface $\left(\left\langle\omega^{+}\right\rangle_{\text {smooth }}\right)$ and rough surface $\left(\left\langle\omega^{+}\right\rangle_{\text {rough }}\right)$. (d) The angular velocity shifts conditioned on the spanwise location: above smooth surface $\left(\left\langle\Delta \omega^{+}\right\rangle_{\text {smooth }}\right)$ and rough surface $\left(\left\langle\Delta \omega^{+}\right\rangle_{\text {rough }}\right)$. We conclude that the plumes originating from the roughness elements lead to enhanced mixing of streamwise momentum, and hence a downwards shift of the velocity profiles. Further away from the wall, the bulk is well-mixed and the streamwise profiles above smooth and rough wall locations converge to a similar value. 
do not exhibit any outer similarity. Deviations of the streamwise and temporal averages from the mean logarithmic profiles are found up to $\Delta \omega^{+} \approx 2$.

For turbulent flows over rough walls, the streamwise velocity profiles retain their logarithmic form. However, the hallmark effect of rough walls is a downwards shift of this region (for any drag increasing surface), which can also be understood as an increase of the skin friction factor $C_{f}$ [8]. Figure 4.9(a) shows the angular velocity profiles $\omega^{+}$as a function of $\left(y^{+}-h_{m}^{+}\right)$, where $h_{m}^{+}$ is the virtual origin and equals the melt-down (i.e. mean) height of the rough surface and $y^{+}=\left(r-r_{i}\right) / \delta_{\nu}$. We choose the melt-down height of the roughness over the full inner cylinder as the virtual origin. In figure 4.9(b) we show the velocity shift versus the wall normal distance. The inset gives a vertical cut at $y^{+}=R e_{\tau}$. It is evident that also in this representation, a maximum in the velocity shift. The position of this maximum $(\tilde{s}=0.61)$ is the same as the one obtained from the angular momentum transport (see section 4.3.2).

In figures Figure 4.9c and Figure 4.9d we employ conditional averaging of the angular velocity profiles over the smooth $\langle\ldots\rangle_{\text {smooth }}$ and rough $\langle\ldots\rangle_{\text {rough }}$ spanwise locations. The wall shear stress in the viscous normalization is taken over the entire spanwise height $L$ of the inner cylinder. We calculate $h_{m}$ also for the rough and smooth patches (where $h_{m \text {,smooth }}=0$ ). We already deduce from figure 4.7 that significant variations in the temporal and streamwise average of the velocity profiles are expected, at least close to the roughness. Indeed we find that for all $\tilde{s}$ the region above the roughness is better mixed, due to the presence of plume-like structures originating from the rough surface. The angular velocity profiles is thus shifted downwards in comparison to the average over the entire IC. For the smooth wall conditioned profiles, we observe the opposite, such that the profiles lay higher.

The merging of plumes from different rough patches into a large scale coherent TTV is also observed in the cross-over of $\left\langle\omega^{+}\right\rangle_{\text {smooth }}$ and $\left\langle\omega^{+}\right\rangle_{\text {rough }}$ for $\tilde{s}=0.61$ in Figure $4.9 \mathrm{~d}$ at $y^{+}-h_{m}^{+} \approx 210$. Further into the bulk flow, turbulent processes mix out the in-homogeneous effects of rough wall attached plumes, and the angular velocity profiles converge to similar values. However, we note that even at $y^{+}-h_{m}^{+}=R e_{\tau},\left\langle\omega^{+}\right\rangle_{\text {smooth }}$ and $\left\langle\omega^{+}\right\rangle_{\text {rough }}$ differ to $\approx 0.5$.

\subsection{Conclusions and outlook}

In conclusion, we have investigated, both numerically and experimentally, large Taylor number Taylor-Couette flow in the presence of spanwise-varying roughness, which consists of an arrangement of stripes of width $\tilde{s}$, that covers 
the entire circumference of the inner cylinder. In the experiments, the stripes were made from sandpaper, while in the numerics a confocal microscopy scan of the surface was implemented by means of the immersed boundary method (IBM).

Remarkably, we have found that by varying $\tilde{s}$ in the range $\tilde{s}=[0.47,3.74]$ we can alter the spanwise wavelength of the turbulent Taylor vortices within the range $\tilde{\lambda} \in[0.94,4.01]$, even if the roughness height was very low $(k / d \approx 0.01)$. This manipulation was observed to hold at $\mathrm{Ta}=\mathcal{O}\left(10^{9}\right)$ and $\mathrm{Ta}=\mathcal{O}\left(10^{12}\right)$.

In the experiments, the scaling of the Nusselt number with the driving strength was found to be effectively $\mathrm{Nu}_{\omega} \propto \mathrm{Ta}^{0.45}$ for $\left.\mathrm{Ta} \in\left[5 \times 10^{11}, 5 \times 10^{12}\right]\right)$.

The experiments and DNSs also revealed that inflow regions $\left(u_{r}<0\right)$ originated between the rough stripes, where the inner cylinder was hydrodynamically smooth (in contrast to secondary flows induced by spanwise-varying roughness in channel flow, where the orientation of the vortices is reversed [116]). Conversely, at the center of the rough stripes, we observed the creation of outflow regions $\left(u_{r}>0\right)$ which were accompanied by the promotion of streamwise velocity fluctuations $\sigma\left(u_{\theta}\right)$ at midgap. At these spanwise locations (center of rough stripes), we observed, in both the numerics and experiments, the emission of plume-like structures, which are responsible for the creation and pinning of the rolls. Since the coverage of the roughness was fixed, we showed that by reducing $\tilde{s}$, we can effectively bring these structures closer, and enhance the interaction of the rolls, as evidenced by the increment in $\left|u_{r}\right|$. As a consequence of this interaction, the flow responded globally by inducing a maximum of angular momentum transport at $\tilde{s}=0.93$ in the experiments, and $\tilde{s}=0.61$ in the numerics.

The numerical simulations and experiments in this chapter fall in the category of low momentum pathways above the roughness and high momentum pathways above the smooth stripes. Note that these are seen in the reference frame of the rotating inner cylinder. These findings agree with the hypothesis that points to a virtual origin effect, since the roughness is protruding and outflow regions are located above the roughness.

For increasing Ta, the relative strength of the turbulent Taylor vortices with respect to the mean flow decreases. Therefore, we find a weaker imprint of the vortices on the angular velocity profiles for PIV measurements at $\mathrm{Ta}=$ $O\left(10^{12}\right)$, than we do for the DNSs at Ta $=O\left(10^{9}\right)$, for smooth walls. Consequently, we also find that the effects of spanwise varying roughness on the flow are somewhat stronger at $\mathrm{Ta}=O\left(10^{9}\right)$ than for $\mathrm{Ta}=O\left(10^{12}\right)$.

We wish to stress that in this study the change in the morphology of the 
large-scale structures is only due to the spanwise-varying roughness (of very low height) and not by a change of $\Gamma$ or $\eta$, which opens the possibility of exploring different configurations in which the rolls can be tuned at such large turbulence levels.

Many questions arise from the aforementioned observations. Understanding the mechanisms leading to the merging of plume ejection regions, and accompanied parameter boundaries at which this occurs, would lead to a further insight into the dynamics of the TTVs. Furthermore, it would be intriguing, in the spirit of [136], to study the influence of spanwise-varying regions of idealized high and low wall shear stress, without geometrical induced disturbances. It is an open question whether one could also alter $\lambda$, without the interaction of the plumes. 


\section{Chapter 5}

\section{Direct numerical simulations of spiral Taylor-Couette turbulence}

We perform direct numerical simulations of spiral turbulent Taylor-Couette (TC) flow for $400 \leq R e_{i} \leq 1200$ and $-2000 \leq R e_{o} \leq-1000$, i.e. counterrotation. The aspect ratio $\Gamma=$ height/gap width of the domain is $42 \leq \Gamma \leq$ 125 , with periodic boundary conditions in the axial direction, and the radius ratio $\eta=r_{i} / r_{o}=0.91$. We show that with decreasing $R e_{i}$ or with decreasing $R e_{o}$, the formation of a turbulent spiral from an initially 'featureless turbulent' flow can be described by the phenomenology of the Ginzburg-Landau equations, similar as seen in the experimental findings of [17] for TC flow at $\eta=0.98$ an $\Gamma=430$ and in numerical simulations of oblique turbulent bands in plane Couette flow by [137]. We therefore conclude that the GinzburgLandau description also holds when curvature effects play a role, and that the finite-wavelength instability is not a consequence of the no-slip boundary conditions at the upper and lower plate in the experiments. The most unstable axial wavelength $\lambda_{z, c} / d \approx 41$ in our simulations differs from findings in Prigent et al. where $\lambda_{z, c} / d \approx 32$, and so we conclude that $\lambda_{z, c}$ depends on the radius ratio $\eta$. Furthermore, we find that the turbulent spiral is stationary in the reference frame of the mean velocity in the gap, rather than the mean velocity of the two rotating cylinders.

Published as: P. Berghout, R.J. Dingemans, X. Zhu, R. Verzicco, R.J.A.M. Stevens, W. van Saarloos, and D. Lohse, Direct numerical simulations of spiral Taylor-Couette turbulence, J. Fluid Mech. 887, A18-1-A18-16 (2020). 


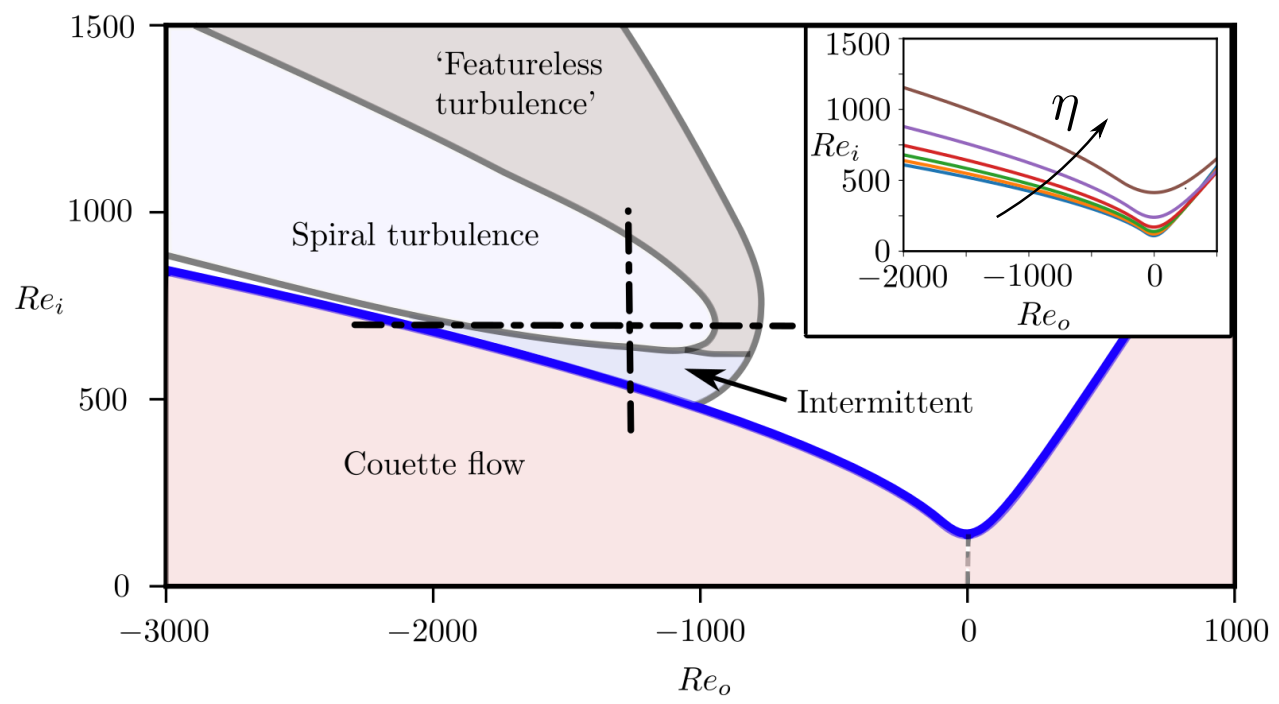

Figure 5.1: Simplified phase space of low Reynolds TC flow. The blue line is the stability boundary at $\eta=r_{i} / r_{o}=0.91$, as considered in this study, calculated with equation (8) of [85]. The intermittent, spiral turbulence, and 'featureless turbulent' regimes are schematics, indicating the approximate locations of the phases at $\eta=0.91$, similar to the phase diagram at lower radius ratio $(\eta=0.84)$ in [138]. The horizontal and vertical dashed-dotted lines represent the simulations that are performed in this chapter. (Inset) Stability boundaries for varying $\eta$. The arrow points towards increasing $\eta$.

\subsection{Introduction}

The coexistence of spatially and/or temporally intermittent turbulent and laminar flow regions is one of the most captivating phenomena in fluid mechanics $[139,140]$. In Taylor-Couette (TC) flow, the flow between two independently rotating concentric cylinders, not too far above the onset of instabilities these patterns manifest themselves as distinctive intertwined bands of laminar and turbulent spirals. Although already observed by [16] and [141], and famously commented on by [142], the origin and dynamics of these patterns remains elusive.

Figure 5.1 presents a simplified phase space of TC flow with inner cylinder Reynolds numbers $R e_{i} \leq O\left(10^{3}\right)$, (where $R e_{i}=u_{i} d / \nu$, with gap width $d$ and inner cylinder velocity $u_{i}$ ) and a counter or co-rotating outer cylinder 
with $-3000 \leq R e_{o} \leq 1000$ (where $R e_{o}=u_{o} d / \nu$, with outer cylinder velocity $u_{o}$ ). In figure 5.1, at high inner cylinder Reynolds number $R e_{i}$, the flow occupies the 'featureless turbulent' state. With decreasing $R e_{i}$, a coherent, spatiotemporal intermittent, turbulent domain appears - spiral turbulence. By further decreasing $R e_{i}$, the spiral structure loses coherence and breaks up into intermittent turbulent spots. Below the Taylor stability boundary [12] the flow becomes entirely laminar. The radius ratio dependence of the stability boundary $R e_{i, c r}$ at which the flow undergoes a transition from laminar to intermittently turbulent was derived by [85], obtaining

$$
\left(\frac{R e_{i}}{R e_{i, l c}}-\frac{R e_{o}}{\eta R e_{i, l c}}\right)^{2} \frac{r_{n}^{2}-r_{p}^{2}}{r_{p}^{2}} \frac{(1+\eta)^{2}}{(1-\eta)(3+\eta)}=f\left(a \frac{d_{n}}{d}\right)^{-4}
$$

where the critical Reynolds number for the case of a resting outer cylinder is $R e_{i, l c}(\eta)=\frac{(1+\eta)^{2}}{2 \eta \alpha^{2}((1-\eta)(3+\eta))^{1 / 2}}$ with $\alpha=0.1556$, and $r_{p}=r_{i}+\frac{d}{2} f\left(a \frac{d_{n}}{d}\right)$, the radius of neutral stability $r_{n}=r_{o}\left(\frac{R e_{i}-\eta R e_{o}}{R e_{i}-\eta^{-1} R e_{o}}\right)^{1 / 2}, d_{n}=r_{n}-r_{i}, a(\eta)=$ $(1-\eta)\left(\sqrt{\frac{(1+\eta)^{3}}{2(1+3 \eta)}}-\eta\right)^{-1}$ and the function $f(x)=x$ if $x<1$ and $f(x)=1$ if $x \geq 1$. (5.1) is shown as the blue line in figure 1. Similar diagrams at different $\eta$ are found in figure 2(a) of [17] and figure 1 in [138].

\subsubsection{Spiral turbulence}

The first studies on spiral turbulence in TC flow go back to [16] and [141], who noticed a 'catastrophic' transition to turbulence if the outer cylinder rotates faster than the inner cylinder. In contrast, for pure inner cylinder rotation they observed a transition by 'spectral evolution', meaning that the complexity of the flow gradually increases with increasing inner cylinder Reynolds number. However, the 'catastrophic' transition does not lead to a 'featureless turbulent' flow directly, but rather forms a state of distinct turbulent and laminar domains, which at specific conditions form regular patterns - spirals. The angular velocity of the spiral was found to be very close to $\omega_{s}=2\left(\omega_{i}+\omega_{o}\right)$, where $\omega_{i}$ and $\omega_{o}$ are the inner and outer cylinder angular velocities, respectively. Furthermore, [141] observed strong hysteresis of the spiral turbulence region when approaching the stability boundary from either the 'featureless turbulence' regime or the Couette flow regime. Later, [143] observed that the pitch angle of the spiral is non-uniform, and showed that this fits well into a framework of phase dynamics, with the phase being represented by the mean 
azimuthal position of the spiral. The boundary conditions at the top and bottom play therein a crucial role.

More recently, the help of direct numerical simulations (DNSs) has led to a further understanding of the fluid flow inside the turbulent structure. [144] discovered that the turbulent spiral originates at the inner cylinder, where vortical structures detach from the wall and spread out radially towards the outer cylinder. For smaller aspect ratios $\Gamma \leq 15$ (with $\Gamma=L / d$ the height of the cylinder divided by the gap width) where no turbulent spiral is formed, turbulent bursts were attributed to a secondary instability mechanism of the laminar flow [145]. We note however that laminar spirals do form at low $\Gamma$, as they also play a central role in the bursting mechanism [146].

By means of conditional averaging over the spiral turbulence domain, [147] revealed that a strong angular gradient of the streamwise velocity prevails in the spiral structure. Subsequently, [148] found that the spiral domain consists of elongated vortical structures and that the linearly unstable region of the laminar flow contains vortices with a streamwise vorticity. Furthermore, [149] simulated spiral turbulence with a stationary inner cylinder, such that the entire flow is linearly stable. Finally, [150] and [151] studied, with the use of DNS, turbulent bands in transitional Plane Couette (PC) flow.

\subsubsection{A pattern forming turbulent spiral}

A new and remarkable insight into the dynamics of the turbulent spiral came from [17]. Their experimental observations of stripes in PC flow and spirals in TC flow at very high radius ratio $\eta=r_{i} / r_{o}=0.98$ reveal a turbulence intensity modulation of these flow states that fits in every respect the phenomenology of the (Complex) Ginzburg-Landau (GL) amplitude equations [152,153]. Thereby, the spiral fits the dynamical behaviour of a finite wavelength instability, originating from the 'featureless turbulent' state. The GL equation describes the time evolution of a complex amplitude $A(x, t)$ of a physical field variable $\mathbf{u}(x, t)$;

$$
\tau_{0} \partial_{t} A(x, t)=\epsilon A+\xi_{0}^{2} \partial_{x}^{2} A-g_{0}|A|^{2} A
$$

where $\tau_{0}$ is the timescale, $\xi_{0}$ is the length scale, and $g_{0}$ sets the magnitude scale of the structure. $\epsilon$ is the reduced bifurcation parameter $\epsilon=\left(R e_{i, c}-R e_{i}\right) / R e_{i, c}$, where $R e_{i, c}$ is the critical inner cylinder Reynolds number at which the bifurcation occurs upon reducing $R e_{i}$ and the pattern emerges. By rescaling equation (5.2), one finds that the intensity of the pattern $|A|^{2}=O\left(\epsilon^{1}\right)$. Note that $[17,154]$ coupled two GL equations to account for the coexistence of spirals 


\subsection{INTRODUCTION}

with opposing helicity and added a noise term, to account for the turbulent fluctuations in the background velocity field.

In principle, for a bifurcation to travelling waves, the coefficients on the right hand side of equation (5.2) are expected to acquire imaginary parts, in other words to be complex. These imaginary parts model the shift of the frequency of the modes with $\epsilon$, wavenumber and amplitude. We will not probe these effects here. In fact, as we shall see, the patterns are actually stationary in the frame moving with the mean flow, which indicates that it may be most appropriate to think of the patterns as stationary. This is natural to expect considering that the equations are invariant under continuous translation in the azimuthal and axial directions.

Further work on the GL description of laminar Taylor spirals has been carried out by [155], who measured the GL coefficients. [137] carried out underresolved simulations of oblique bands in Couette flow. Also the amplitude in their simulations, defined as the modulus of the first Fourier mode of the streamwise velocity, does obey the phenomenology of the GL model.

In this chapter, we set out to investigate spiral turbulent TC flow by means of DNS. In contrast to the experiments of [17], which were carried out in the limit of very small curvature $(\eta=0.98)$, in our DNSs, curvature effects do play a role $(\eta=0.91)$. To quantify this, we refer to the curvature Obukhov length $L_{c}=\frac{u_{\tau}}{\kappa \omega_{i}}$, with $u_{\tau}$ the friction velocity and $\kappa=0.39$ the von Kármán constant, as defined in [14]. This length differentiates the flow regions in a turbulent flow where the production of turbulent kinetic energy is governed by shear and in one where it is governed by curvature of the streamlines. For values above roughly $\left(r-r_{i}\right) / L_{c} \geq 0.1$, the effects of curvature are pronounced. We find that for $\eta=0.91,0.5 d / L_{c} \approx 0.2$. For $\eta=0.98,0.5 d / L_{c} \approx O\left(10^{-3}\right)$.

Further, we employ periodic boundary conditions in the axial direction, and thereby exclude effects originating from the end plates which are responsible for the no-slip axial boundary conditions in experiments. We study both the fluid mechanics of the turbulent spirals and investigate whether the GL description of the spirals holds in our simulations.

The chapter is organized as follows: In section 5.2 we present the description of TC flow and the details of the numerical solver. In section 5.3.1 we study the appearance and disappearance of the spiral structure, followed by section 5.3 .2 , in which we look at the impact of the turbulent spiral on the global transport of momentum. In section 5.3 .3 we study the velocity of the spiral pattern. Section 5.3.4 then presents the GL description of the spiral. The chapter ends with conclusions (section 5.4). 


\subsection{Taylor-Couette flow and numerical procedure}

The TC setup consists of two concentric, independently rotating cylinders with radii $r_{i}$ and $r_{o}$. The gap width $d$ is defined as $d=r_{o}-r_{i}$, the radius ratio $\eta=r_{i} / r_{o}$, and the aspect ratio as $\Gamma=L / d$, where $L$ is the height of the cylinders. In this chapter we keep the radius ratio fixed at $\eta=0.91$. The aspect ratio $\Gamma$ varies in between $42 \leq \Gamma \leq 125$. For every DNS we simulate the full azimuthal circumference of the TC setup.

The Navier-Stokes equations that govern the shear-driven fluid flow in between the two concentric rotating cylinders are formulated in cylindrical coordinates and dimensionless form, namely

$$
\begin{gathered}
\partial_{\hat{t}} \hat{u}_{r}+\hat{\mathbf{u}} \cdot \hat{\nabla} \hat{u}_{r}-\frac{\hat{u}_{\theta}^{2}}{\hat{r}}=-\partial_{\hat{r}} \hat{P}+\frac{1}{R e_{i}-R e_{o} \eta}\left(\hat{\nabla}^{2} \hat{u}_{r}-\frac{\hat{u}_{r}}{\hat{r}^{2}}-\frac{2}{\hat{r}^{2}} \partial_{\hat{\theta}} \hat{u}_{\theta}\right) \\
\partial_{\hat{t}} \hat{u}_{\theta}+\hat{\mathbf{u}} \cdot \hat{\nabla} \hat{u}_{\theta}+\frac{\hat{u}_{r} \hat{u}_{\theta}}{\hat{r}}=-\frac{1}{\hat{r}} \partial_{\hat{\theta}} \hat{P}+\frac{1}{R e_{i}-R e_{o} \eta}\left(\hat{\nabla}^{2} \hat{u}_{\theta}-\frac{\hat{u}_{\theta}}{\hat{r}^{2}}+\frac{2}{\hat{r}^{2}} \partial_{\hat{\theta}} \hat{u}_{r}\right) \\
\partial_{\hat{t}} \hat{u}_{z}+\hat{\mathbf{u}} \cdot \hat{\nabla} \hat{u}_{z}=-\partial_{\hat{z}} \hat{P}+\frac{1}{R e_{i}-R e_{o} \eta}\left(\hat{\nabla}^{2} \hat{u}_{z}\right) \\
\hat{\nabla} \cdot \hat{\mathbf{u}}=0
\end{gathered}
$$

The differential operators are defined as: $(\mathbf{u} \cdot \nabla) f=\left(u_{r} \partial_{r}+u_{\theta} \frac{1}{r} \partial_{\theta}+u_{z} \partial_{z}\right) f$ and $\nabla^{2} f=\frac{1}{r} \partial_{r}\left(r \partial_{r} f\right)+\frac{1}{r^{2}} \partial_{\theta}^{2} f+\partial_{z}^{2} f$. The boundary conditions are $\left.u_{\theta}\right|_{r_{i}}=r_{i} \omega_{i}$ and $\left.u_{\theta}\right|_{r_{o}}=r_{o} \omega_{o},\left.\left(u_{z}, u_{r}\right)\right|_{r_{i}}=0$ and $\left.\left(u_{z}, u_{r}\right)\right|_{r_{o}}=0$. In the axial direction we employ periodic boundary conditions. $R e_{i}=r_{i} \omega_{i} d / \nu$ and $R e_{o}=r_{o} \omega_{o} d / \nu$ are the inner and outer cylinder Reynolds numbers, respectively, where by definition $\omega_{i} \geq 0$. Note that the equations can also be written in terms of the Taylor number and a geometric Prandtl number, highlighting the analogy with Rayleigh-Bénard convection [13]. The velocity vector $\mathbf{u}$ comprises $\left(u_{\theta}, u_{z}, u_{r}\right)$, respectively the streamwise, spanwise and wall-normal velocity, which in this chapter are normalized by the inner cylinder azimuthal velocity $u_{\theta, i}$. Hatted symbols represent dimensionless variables, where velocity, length and time are made dimensionless as, respectively, $u=r_{i}\left|\omega_{i}-\omega_{o}\right| \hat{u}, r=d \hat{r}$ and $t=\frac{d}{r_{i}\left|\omega_{i}-\omega_{o}\right|} \hat{t}$ and pressure is made dimensionless accordingly, $P=\rho r_{i}^{2}\left|\omega_{i}-\omega_{o}\right|^{2} \hat{P}$.

The equations are spatially discretized to second order and are solved on a finite difference grid [50]. Time integration is performed with a fractional-step third-order Runge-Kutta scheme. For more details of the numerical code we refer to [51] and [156]. Grid independence checks are carried out to ensure sufficient numerical resolution. Time convergence is controlled by monitoring 
the torque on both cylinders, which is expressed in dimensionless form as the Nusselt number $N u_{\omega}$,

$$
N u_{\omega}=\frac{J^{\omega}}{J_{\text {lam }}^{\omega}}=\frac{\mathcal{T}(2 \pi L \rho)^{-1}}{2 \nu r_{i}^{2} r_{o}^{2} \frac{\omega_{i}-\omega_{o}}{r_{o}^{2}-r_{i}^{2}}}
$$

where $J^{\omega}=r^{3}\left(\nu \partial_{r}\langle\omega\rangle_{A(r), t}+\left\langle u_{r} \omega\right\rangle_{A(r), t}\right)$ is the angular velocity flux, and $\langle.\rangle_{A(r), t}$ represents averaging over a cylinder surface $A(r)$ and time $t, J_{\text {lam }}^{\omega}=$ $2 \nu r_{i}^{2} r_{o}^{2} \frac{\omega_{i}-\omega_{o}}{r_{o}^{2}-r_{i}^{2}}$ is the laminar angular velocity flux and $\mathcal{T}$ is the torque. Alternatively to $N u_{\omega}$, we define the friction factor $C_{f}$;

$$
C_{f}=\frac{G}{\left(R e_{i}-\eta R e_{o}\right)^{2}}=\frac{2 \pi N u_{\omega} J_{\mathrm{lam}}^{\omega}}{\nu^{2}\left(R e_{i}-\eta R e_{o}\right)^{2}}
$$

where $G=\mathcal{T} /\left(\rho \nu^{2} L\right)$ is the dimensionless torque. $C_{f}$ is thus a ratio of the wall stress over the kinetic energy of the flow.

\subsection{Results and discussion}

\subsubsection{Turbulent spirals: Their formation and disappearance}

When the destabilizing effects of inner cylinder rotation become large compared to the stabilizing effects of outer cylinder rotation, TC flow can enter the 'featureless turbulent' regime, see figure 5.1. Although the flow is turbulent throughout the domain, the term 'featureless turbulence' as mentioned in [138] is deceiving, since the mean flow does contain structures with streamwise vorticity, i.e. the Turbulent Taylor Vortex (TTV) [101].

We run a DNS of the 'featureless turbulent' regime, with $R e_{i}=800$ and $R e_{o}=-1200$, of which 3 snapshots of the streamwise/azimuthal, velocity at varying radii are presented in figure 5.2 (top row). As initial conditions for all our simulations we use the laminar solution of TC flow [13] plus small spatial perturbations to the radial velocity component $u_{r}=0.1 r_{i} \omega_{i} \sin (2 \pi z / L)$, with $z$ the axial coordinate and the axial velocity component $u_{z}=0.1 r_{i} \omega_{i} \sin ((r-$ $\left.\left.r_{i}\right) 2 \pi / d\right)(1-|\sin (2 \pi z / L)|) \sin (2 \pi z / L)$. The resolution is set to $N_{\theta} \times N_{z} \times N_{r}=$ $768 \times 1280 \times 80$ at $\Gamma=64$, such that we resolve the global Kolmogorov scale $\eta_{k}=0.03 d$, where $\eta_{k}=\left(\frac{\nu^{3}}{\epsilon_{\nu}}\right)^{1 / 4}$, with $\epsilon_{\nu}$ the mean kinetic dissipation rate. The spacing of the finite size grid at the wall in the radial direction is $0.44 y_{0}$, where $y_{0}$ is the viscous length scale $y_{0}=\nu / \sqrt[\hat{a} \text { Áćc }]{\tau_{w} / \rho}$, with $\nu$ the kinematic velocity, $\rho$ the fluid density, and $\tau_{w}$ the wall shear stress. With varying $\Gamma$ we change $N_{z}=1280(\Gamma / 64)$ while $N_{\theta} \times N_{r}=768 \times 80$ for all simulations. 

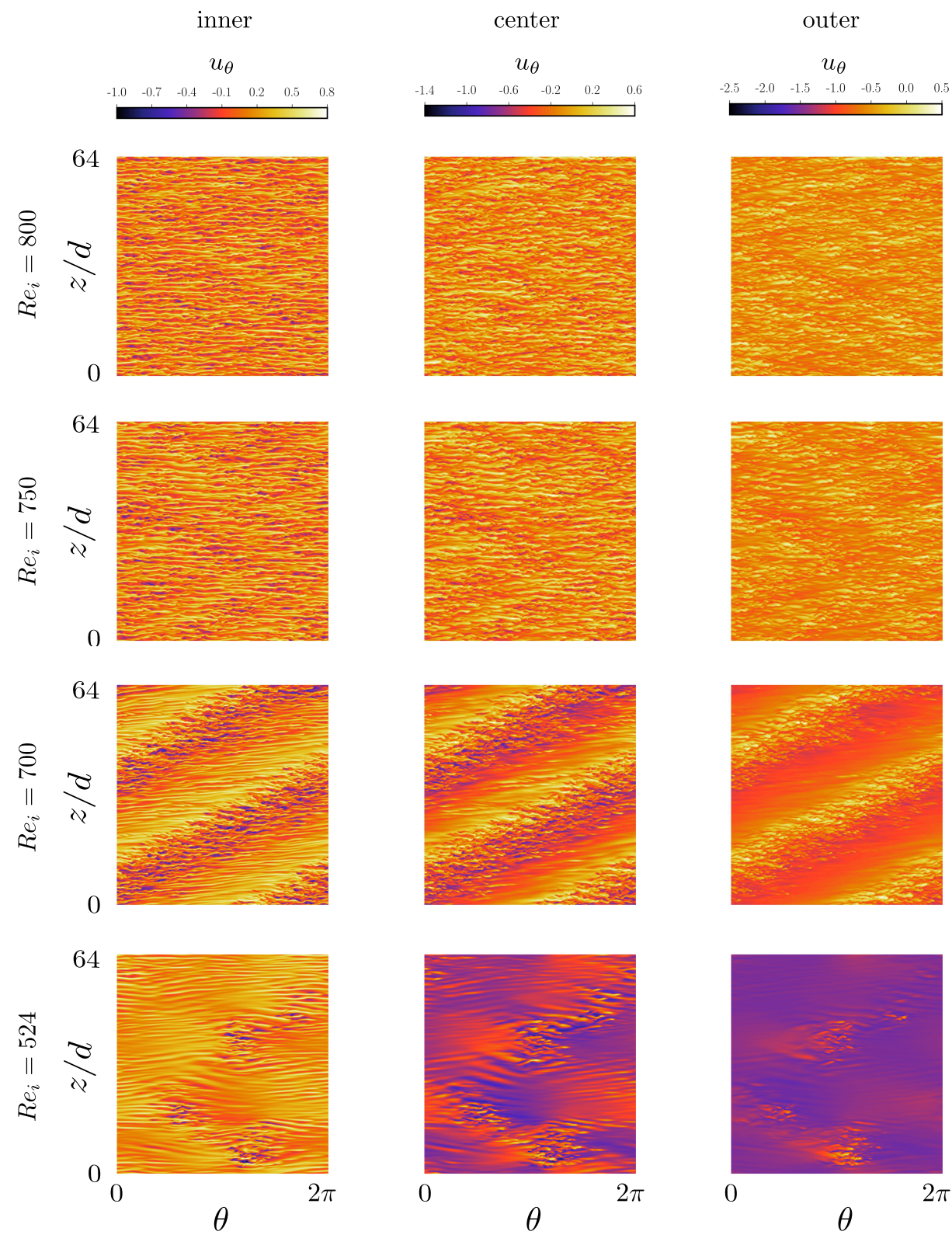

Figure 5.2: Snapshots of the azimuthal velocity $u_{\theta}$, close to the inner cylinder $\left(r=r_{i}+d / 4\right)$, at the center $\left(r=r_{i}+d / 2\right)$ and close to the outer cylinder $\left(r=r_{i}+3 d / 4\right)$ for $R e_{o}=-1200$ and for different $R e_{i}$. From a chaotic turbulent base flow (upper row), the finite wavelength instability forms (second and in particular third row). Further away from the transition an isolated stripe (spiral) breaks down in connected and isolated turbulent spots (fourth row). 


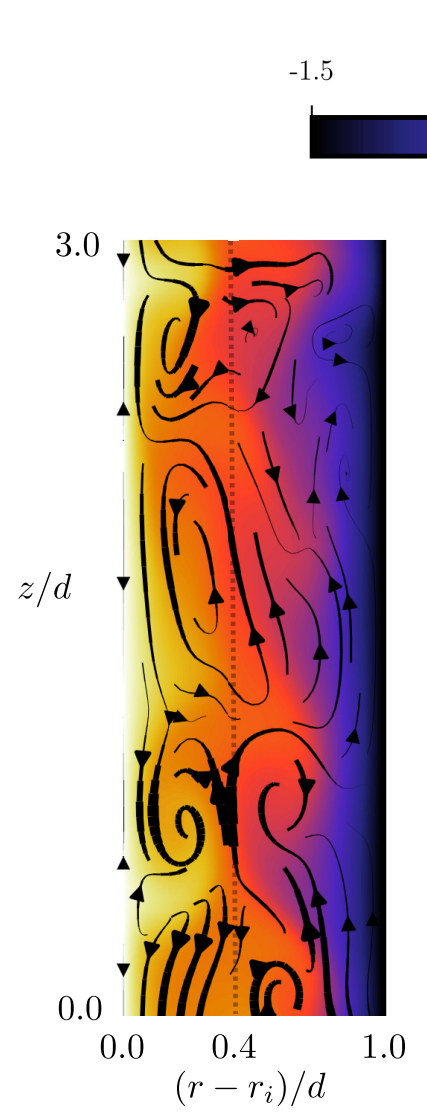

(a) $R e_{i}=800$

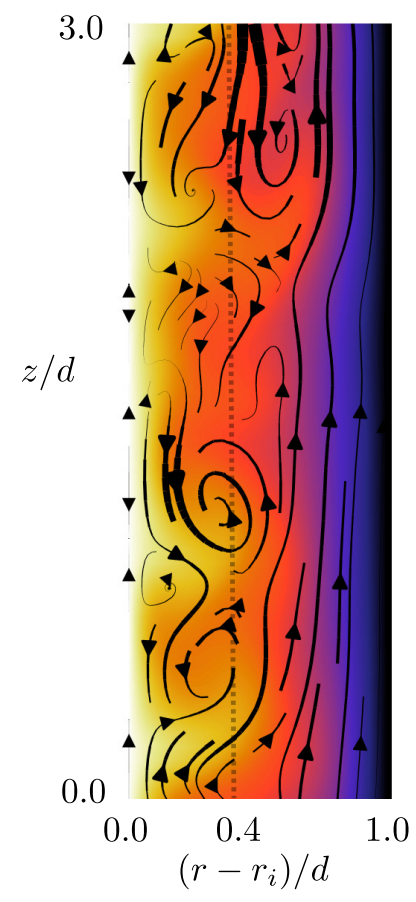

(b) $R e_{i}=750$

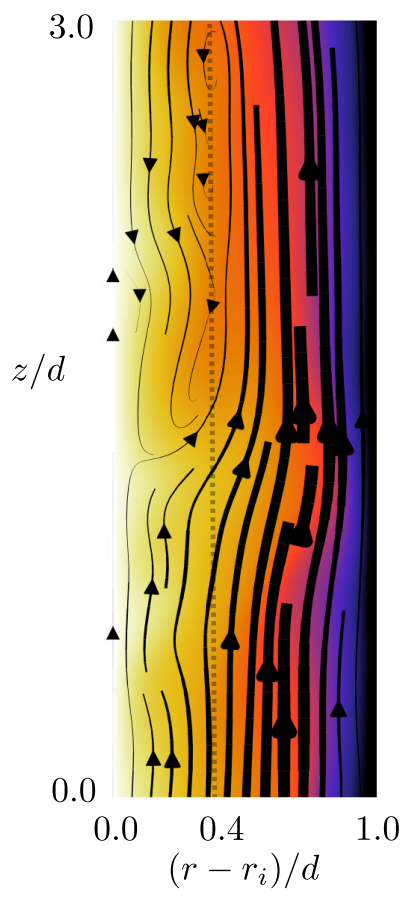

(c) $R e_{i}=700$

Figure 5.3: Streamlines overlay snapshots of the azimuthal velocity $u_{\theta}$ in the meridional plane for $R e_{o}=-1200$. The thickness of the streamlines represents the norm of the velocity vector $\left(u_{r}, u_{z}\right)$. We observe chaotic motion for all $r$ in the 'featureless turbulent' flow $(a)$. With decreasing $R e_{i}$, laminarization occurs from the outer cylinder towards the inner cylinder $(b, c)$. The vertical dashed lines at $\left(r-r_{i}\right) / d \approx 0.4$ give the location of the nodal plane of neutral stability. The meridional snapshots where obtained at $\theta=\pi$. 
In figure 5.2 we observe long, thin, meandering patterns in the azimuthal velocity $u_{\theta}$. The structures have a length scale $\approx(0.5-1.0) d$ in the axial direction and are more distinct close to the inner cylinder at $r=r_{i}+0.25 d$, whereas they become more diffused closer to the outer cylinder at $r=r_{i}+$ $0.75 d$. The nodal plane of neutral stability (defined by the laminar azimuthal velocity being zero $u_{\theta, \text { lam }}=0$ ), lays at $r_{n}=r_{o} \sqrt{\frac{R e_{i}-\eta R e_{o}}{R e_{i}-\eta^{-1} R e_{o}}} \approx 1.04 r_{i}$ for $R e_{i}=$ 800 and $R e_{o}=-1200$, such that the inner region of the domain $r<1.04 r_{i}$ is linearly unstable and the outer region of the domain $r>1.04 r_{i}$ is linearly stable [78]. This is confirmed in figure 5.3(a), where we present contours of the instantaneous azimuthal velocity $u_{\theta}=u_{\theta}(\theta, z, r, t)$ in a small portion of the axial-radial plane at $R e_{i}=800$ and $R e_{o}=-1200$. We find vortical structures, with a streamwise vorticity and length scale $\approx(0.5-1.0) d$ close to the inner cylinder. These structures resemble the Taylor vortices, although they do not close the entire circumferential direction of the domain, see figure 5.2. The region $r>r_{n}$ does exhibit chaotic fluid flow motion, triggered by the instabilities from the inner region. This is reminiscent of the so-called inner-outer region interaction as described by [145]. As such, we find that for $R e_{i}=800$ the entire domain is filled with chaotic (turbulent) fluid motion, and hence falls into the 'featureless turbulent' part of the above phase space, see figure 5.1.

Starting from this state (figure 5.2, upper row), we decrease $R e_{i}$ to $R e_{i}=750$ (figure 5.2, second row), while keeping $R e_{o}=-1200$. We run the simulations until the flow is statistically stationary, i.e. when the dimensionless torque $\left\langle N u_{\omega}\right\rangle_{t}$, calculated at both cylinder, is constant to within $1 \%$, where $\langle.\rangle_{t}$ indicates time averaging over $50 \hat{t}$. Resulting from the decrease in shear, the flow starts to laminarize at the outer cylinder, see figure 5.3(b). However, the flow remains turbulent throughout the majority of the domain, as seen in figure 5.2 (second row). Interestingly, we find in these turbulent parts of the flow, in the contours of $u_{\theta}(\theta, z, r, t)$, appearances of diagonal coherent patterns. At this $R e_{i}$ the patterns exist at opposing angles and 'nucleate' at varying places.

Subsequently, we further lower $R e_{i}$ to $R e_{i}=700$ (figure 5.2, third row) for which one well-defined turbulent spiral pattern emerges. At $r=r_{i}+0.75 d$, the spiral contains turbulent motion, whereas the region in between the turbulent spiral bands is laminarized and contains no vorticity, see figure 5.3(c). Note that the regions of maximal intensity of the banded structure is shifted to the right somewhat as one moves outwards (this is most clearly visible for $\left.R e_{i}=700\right)$. This is in agreement with the results of [144] for the structure of turbulent bands. Close to the inner cylinder, the spiral also contains 
turbulent motions, however the region in between the turbulent spiral bands does contain the Taylor-like vortices. Figure 5.3(c) presents a section of the turbulent spiral corresponding to $0 \leq z / d \leq 3$ in figure 5.2 (third row). For an extensive range of $530 \leq R e_{i} \leq 700$, the spiral remains present in the flow. Lowering $R e_{i}$ even further, e.g. to $R e_{i}=524$ (see figure 5.2 (bottom row)), makes the spiral lose coherence. The flow then contains intermittent 'puffs' of chaotic motion in an otherwise laminar base flow. We have carried out several numerical simulations around $R e_{i}=524$, namely with $R e_{i}=[490,500,510,520,522,524,526,528,530]$ in order to locate the critical Reynolds number $R e_{i, c r i t}$ at which the turbulence can be sustained. For simulation times as long as $T \approx 1000 \frac{d}{r_{i}\left(\omega_{i}-\omega_{o}\right)}$, we find that the puffs are only sustatined at $R e_{i} \geq 524$. The dynamics of the spatio-temporal intermittent, incoherent, 'puffs' are however not the focus of this study. In the remainder we will study the dynamics in that part of the phase space where the spiral is still coherent.

\subsubsection{Dynamics of the turbulent spiral}

The influence of the turbulent spiral structure on the momentum transport is not addressed in the literature. In this section we will study the global response for both varying spiral wavelength $\lambda_{z}$ and varying $R e_{i}$, while we keep $R e_{o}=-1200$. To vary $\lambda_{z}$, we simulate 20 cases of varying aspect ratio $42 \leq \Gamma=L / d \leq 125$ of the TC setup. In the axial direction we employ periodic boundary conditions, such that $\lambda_{z}=\Gamma / n$, with $n$ a positive integer that represents the number of windings of the spiral around the inner cylinder. We find a range of $26 \leq \lambda_{z} / d \leq 45$, whereby up to 3 spirals fit into the domain for a given $\Gamma$. When we examine the mean velocity and the mean turbulent intensity, we do not find any influence of $\Gamma$ on the flow for fixed $\lambda_{z} / d$. In other words, the flow at $\Gamma=84$ with 2 spirals of $\lambda_{z} / d=42$ is statistically identical to the flow at $\Gamma=42$ with 1 spiral of the same $\lambda_{z} / d=42$.

There is, however, a strong dependence of the global angular momentum transport - expressed in dimensionless form in equation (5.7) - on the axial wavelength of the spiral. Figure 5.4(a) presents $N u_{\omega}$ versus $\lambda_{z} / d$ for varying $R e_{i}$. With increasing $\lambda_{z} / d$, the turbulence fraction in the domain decreases, resulting in a predominantly diffusive, less efficient, transport of momentum. Figure 5.4(b) presents the corresponding $C_{f}$ versus $\lambda_{z} / d$, see equation 5.8.

The graphical representation of $C_{f}\left(R e_{i}\right)$ is commonly referred to as the 'Moody' diagram [37]. Whereas the laminar part of the diagram can be derived analytically, the turbulent part is empirically fitted with the celebrated Prandtl's 

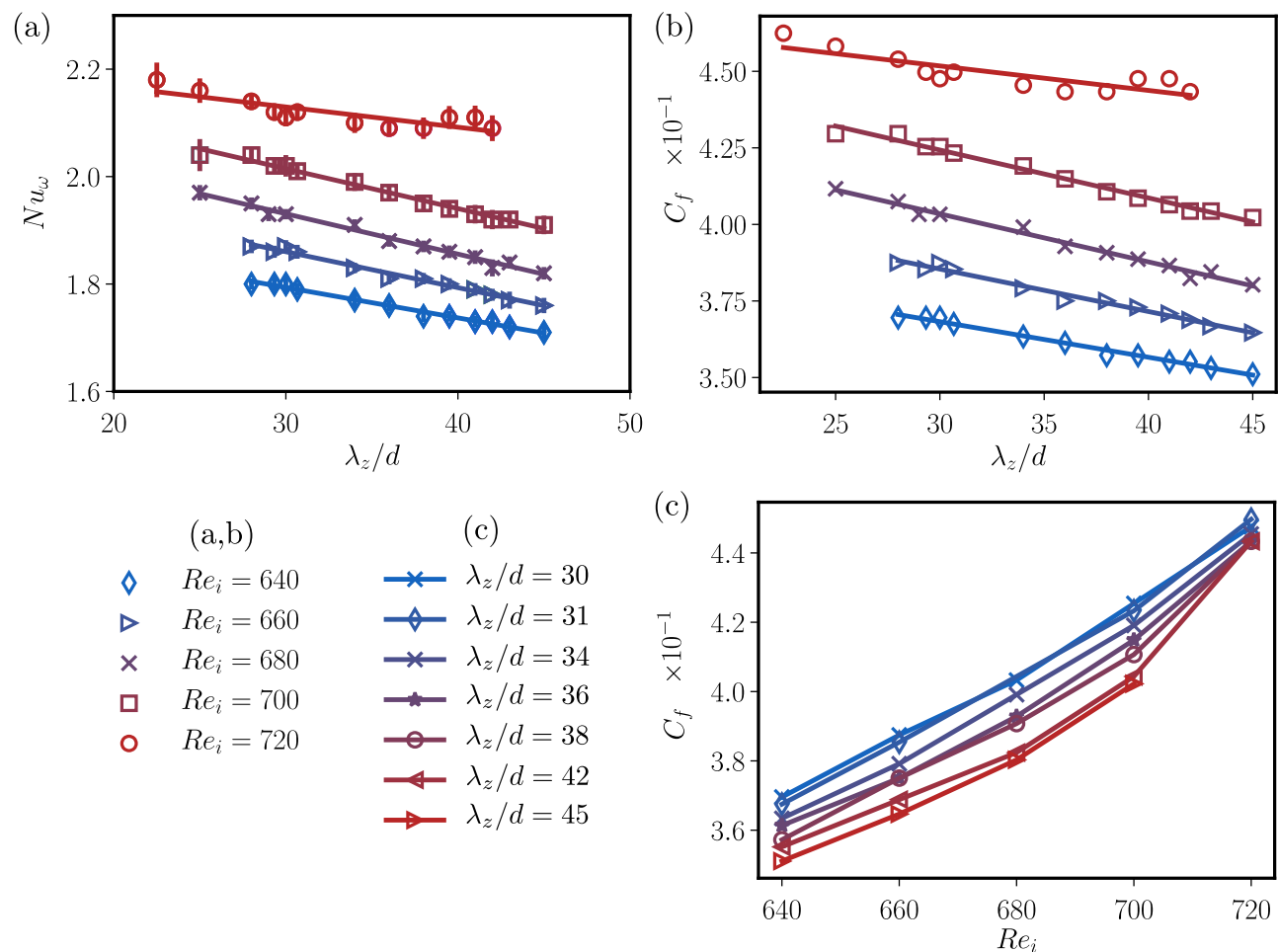

Figure 5.4: (a) Dimensionless angular velocity transport $N u_{\omega}$ versus the wavelength $\lambda_{z} / d$ of the turbulent spiral for different Reynolds. $N u_{\omega}$ decreases linearly with increasing wavelength, which is attributed to the decrease in turbulence fraction in the domain, with increasing axial wavelengths of the spiral. (b) The friction factor $C_{f}$ for different Reynolds versus $\lambda_{z} / d$. (c) $C_{f}$ versus the inner cylinder Reynolds number $R e_{i}$ for $R e_{o}=-1200$. An increase of $C_{f}$ indicates transitional behaviour from the laminar to the turbulent regime. 


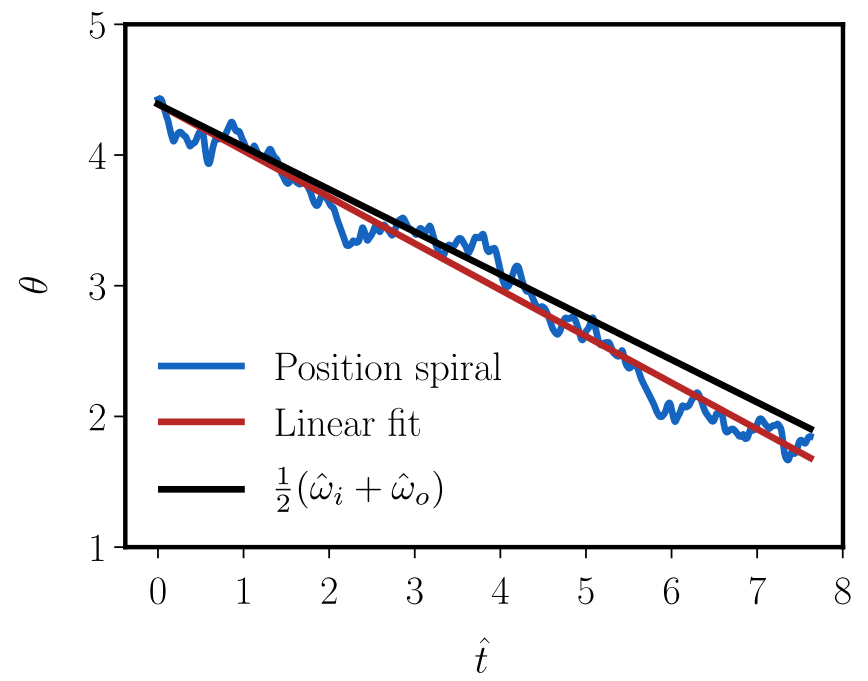

Figure 5.5: Angular location $\theta$ of the turbulent spiral versus time $\hat{t}$. The vertical axis represents the angular position of the maximum turbulent intensity at $z=L / 2$ and $r=r_{i}+d / 2$. The horizontal axis represents dimensionless time $\hat{t}=t / T$. $\langle\hat{\omega}\rangle_{r, \theta, z}$ is the calculated mean angular velocity in the domain. Excellent agreement between $\Delta \theta / \Delta \hat{t}=-0.355$ and $\langle\hat{\omega}\rangle_{r, \theta, z}=-0.354$ reveals that the spiral is stationary in the reference frame of the mean angular velocity $\langle\hat{\omega}\rangle_{\theta, z, r, t}$. Note that $\frac{1}{2}\left(\hat{\omega}_{i}+\hat{\omega}_{o}\right)=-0.326$, solid black line, does not match $\Delta \theta / \Delta \hat{t}$.

friction law. For inner cylinder rotation, linearly unstable, TC flow, $C_{f}\left(R e_{i}\right)$ is monotonically decreasing in the transition region between the laminar and turbulent flow [49]. For counter-rotating TC flow, where the transition to turbulence is subcritical and 'catastrophic', we find that $C_{f}\left(R e_{i}\right)$ is increasing in the transitional region, see figure 5.4(c). This is reminiscent to the transition scenario in pipe flow, where the sudden appearance of spatio-temporally intermittent turbulence leads to an increase in $C_{f}(R e)$ [11].

\subsubsection{Angular velocity of the spiral}

Surprisingly, the angular velocity of the spiral $\omega_{s}$ has hitherto got only little attention in literature. [141] was the first to investigate $\omega_{s}$ and found that it scales with the mean angular velocity of the two cylinders $\omega_{s} \approx \frac{1}{2}\left(\omega_{i}+\omega_{o}\right)$ for $R e_{i}=O\left(10^{3}\right), R e_{o}<-10^{4}$. Later, [138] investigate $\omega_{s}$ for much lower 

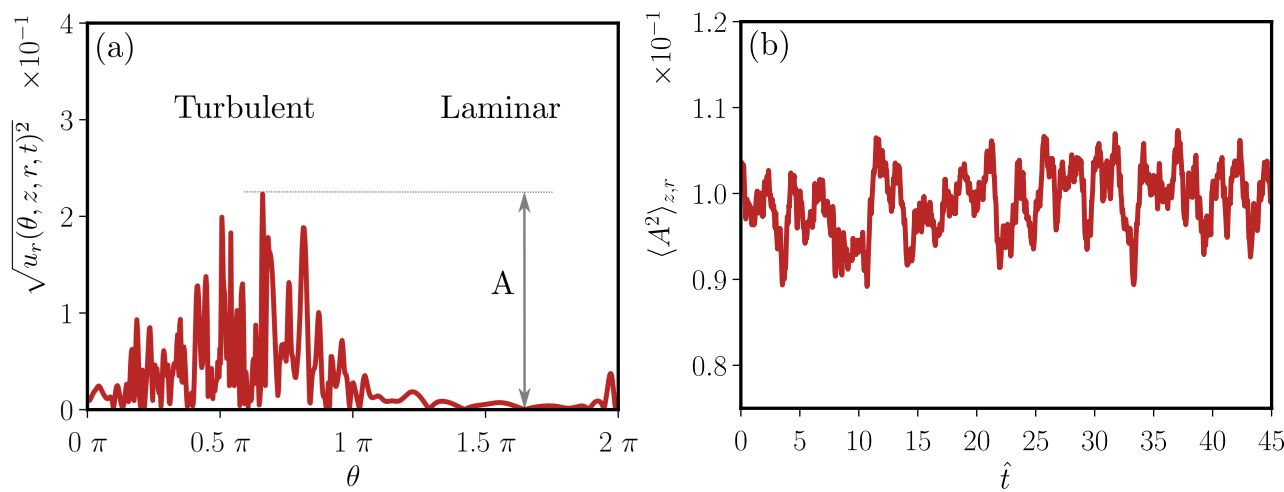

Figure 5.6: Definition of the amplitude $A$. (a) plot of the square root of the radial velocity component squared $\sqrt{u_{r}(\theta, z, r, t)^{2}}$ versus the angular position at $r=r_{i}+d / 2$ and $z=L / 2$ at an arbitrary instant in time when the flow is statistically stationary. The amplitude is defined as $A(z, r, t)=$ $\left(\sqrt{u_{r}(z, r, t)^{2}}\right)_{\max }-\left(\sqrt{u_{r}(z, r, t)^{2}}\right)_{\min }$. (b) The time dependent signal. To obtain the converged amplitude, we employ time averaging and we average over spatial coordinates $\left(r_{i}+d / 4\right)<r<\left(r_{i}+3 d / 4\right)$ and for all $z$. The particular signal is acquired for $R e_{i}=660$ and $R e_{o}=-1200$.

$R e_{i}=O\left(10^{3}\right), R e_{o}=-3500$, and find $\omega_{s} \approx \omega_{o}$. For laminar interpenetrating spirals [155] find that $\omega_{s}=\omega_{o}$. However, the analysis of [157] (for similar values of $R e_{i}, R e_{o}$ as in this work) shows that $\omega_{s}=0.98 \omega_{m}-0.02 \omega_{i}$, where $\omega_{m}=\frac{1}{2}\left(\omega_{i}+\omega_{o}\right)$. In our present DNSs we have access to the full velocity field, in contrast to the experimental work. Thereby we monitor the position of the maximum turbulence intensity in the spiral and from the translation of that position in time extract $\omega_{s}$, see figure 5.5. We find that $\omega_{s}$ is not equal to the mean rotation rate, $\frac{1}{2}\left(\omega_{i}+\omega_{o}\right)$, but instead equals the mean angular velocity in the domain $\langle\omega\rangle=\langle\omega\rangle_{\theta, z, r, t}$. Note that the difference between $\langle\omega\rangle$ and $\omega_{s}$ is only minor and could easily be missed in experiments. In fact, we think that the consistent mismatch between $\omega_{m}$ and $\omega_{s}$, as found in figure 5 in [157], is explained by the mismatch between $\omega_{m}$ and $\langle\omega\rangle$.

\subsubsection{Amplitude modulation}

\section{The amplitude}

To describe the turbulent spiral as a pattern forming instability above a critical bifurcation point $\epsilon=0$, we introduce a perturbation $A(z, t) e^{(i k z-i \omega t)}$ to the 

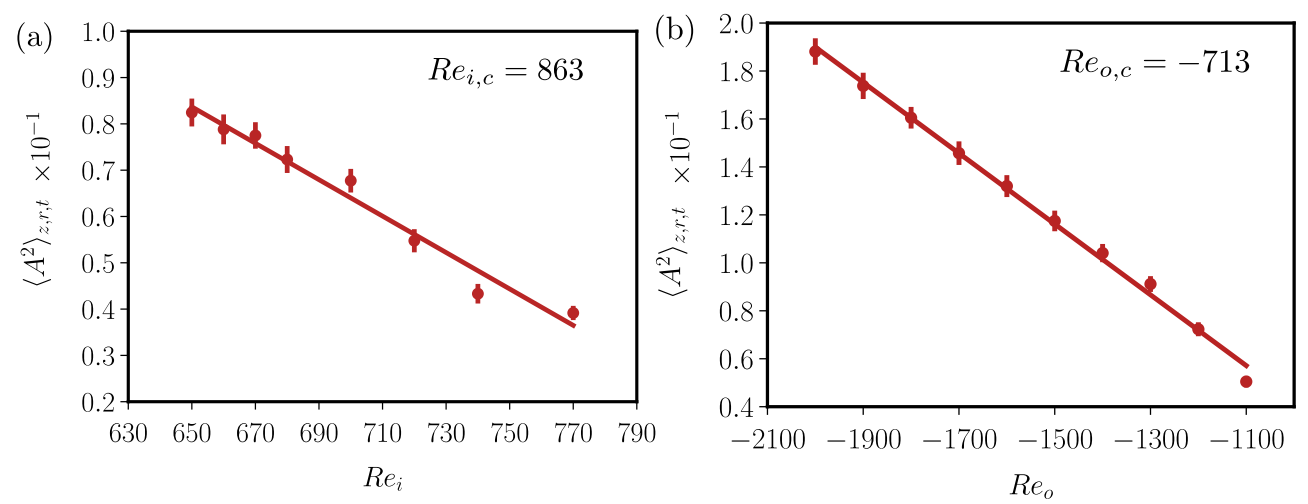

Figure 5.7: Amplitude scaling of the turbulent spiral with varying $\epsilon$. (a) Scaling of the amplitude squared $A^{2}$ with varying $R e_{i}\left(R e_{o}=-1200\right)$. The fit highlights that $A^{2} \propto \epsilon^{1}$, with $\epsilon=\left(R e_{i, c}-R e_{i}\right) / R e_{i, c}$, as predicted by the GL equations. Extrapolation of the fit gives $R e_{i, c}=863$, which compares very well with the experimental results in [17] for $\eta=0.98$. (b) Scaling of $A^{2}$ with varying $\operatorname{Re}_{o}\left(R e_{i}=680\right)$. We observe an identical scaling for $A^{2} ; A^{2} \propto \epsilon^{1}$, now with $\epsilon=\left(R e_{o, c}-R e_{o}\right) / R e_{o, c}$ and $R e_{o, c}=-713$. Error bars represent the standard deviation of $\left\langle A^{2}\right\rangle_{z, r}(t)$.

base flow $u_{b}$. Here we treat the instability in the axial coordinate direction only. Note that the instability contains both parity symmetry (flipping of the streamwise or axial coordinate directions) and translational symmetry. We define the perturbed physical field $u$ as the root mean square (rms) of the radial velocity component (in contrast to [17], who define $u$ as the rms of the axial velocity).

Figure 5.6(a) exhibits the instantaneous $u_{r, r m s}$ over a line encircling the inner cylinder at $r=r_{i}+0.5 d$ and $z=L / d$. We define the amplitude from the radial component of velocity $u_{r}$ as $A=\left(\sqrt{u_{r}^{2}}\right)^{\text {max }}-\left(\sqrt{u_{r}^{2}}\right)^{\text {min }}$ which is a function of $(r, z ; t)$. We calculate $A$ at runtime and average over half the gap width $\left(r_{i}+d / 4\right)<r<\left(r_{i}+3 d / 4\right)$ and the full height $z$. Figure 5.6(b) presents the time signal of the spatially averaged squared amplitude $\left\langle A^{2}\right\rangle_{z, r}$. Significant fluctuations of $\left\langle A^{2}\right\rangle_{z, r}$ force us to also take long averages of $\hat{\tau}_{a v} \approx 50$. The width of the turbulent spiral $\Delta_{s}$ is approximately $\left(r_{i}+0.5 d\right) \pi$.

We measure the amplitude versus the bifurcation parameter $\epsilon$ at a fixed aspect ratio $\Gamma=64$, and hence a fixed $\lambda_{z} / d=32$. For a stationary, finite wavelength instability, of which the modulation of the amplitude is described by equation (5.2), we expect $A^{2} \propto \epsilon^{1}$ [153]. Indeed, this scaling is found by [17], who 
(for different $\eta=0.98$ ) extracted a critical $R e_{i, c}=857 \pm 5$ at which the instability occurs. Figure 5.7(a) shows that we also observe $A^{2} \propto \epsilon^{1}$ over a range of $\epsilon$, close to the bifurcation point. With $R e_{o}=-1200$ we obtain $R e_{i, c}=863$, in very close agreement with [17], in spite of different $\eta$. For $R e_{i}>780$, nucleation of domains of opposing helicity, and the appearance of turbulence in the laminar spiral regions, obscures the precise measurements of the amplitude of the turbulence intensity signal. [17] were still able to extract the amplitude for these Re due to very long runtime in experiments, which at these $\Gamma$ are not accessible for DNS. As for $R e_{i}=770$ the amplitude is still 0.04 , we conclude that the noise term is certainly high.

In a similar manner, we also approach the boundary of the spiral turbulent regime in figure 5.1 from the horizontal direction, i.e. by varying $R e_{o}$ and maintaining $R e_{i}=680$. For decreasing $R e_{o}$, the stabilizing stratification of centrifugal pressure increases, the flow laminarizes and the spiral emerges. As such, we define $\epsilon=\left(R e_{o, c}-R e_{o}\right) / R e_{o}$. Figure 5.7(b) convincingly indicates that also for varying $R e_{o}, A^{2} \propto \epsilon^{1}$, with $R e_{o, c}=-713$. This indicates that the turbulent spiral behaves as a finite wavelength instability, in spite of the curvature effects.

\section{The instability diagram}

By carefully varying the aspect ratio $\Gamma$ we are able to study a variation in the axial wavelength $\lambda_{z}$ of the spiral in the domain $26 \leq \lambda_{z} / d \leq 45$. Considering that we do probe the amplitude of a stationary turbulent spiral versus the wavelength, and the influence of the wavelength of the momentum transport, we do not imply that for a certain aspect ratio only one such wavelength exists. In fact, it is most likely that the wavelength of the spiral is sensitive to initial conditions, like also the wavelength of the Taylor-Vortex is. With axially periodic boundary conditions, we do not find any Reynolds number dependence of $\lambda_{z}$, neither on $R e_{i}$ nor on $R e_{o}$, since $\lambda_{z}=\Gamma / n$. In contrast, such a dependence was experimentally observed by [157] for no-slip boundary conditions at the plates and a much larger system $\Gamma=430$. Figure 5.8 presents the amplitude versus the wavenumber $k=2 \pi / \lambda_{z}$ for increasing $\epsilon$. Note that $R e_{i}$ and $R e_{o}$ are identical to those in figure 4 , except for $R e_{i}=720$ which is excluded here due to very high noise originating from the 'featureless' turbulent flow.

The parabolic shape, with a maximum around $\lambda_{z, c} / d=41 \pm 2$, represents the characteristic band of unstable wavenumbers for a finite wavelength instability above onset. Thereby we do observe the dependence of $A$ on the bifurcation parameter $\epsilon$, such that an increasing band of wavenumbers becomes unstable 


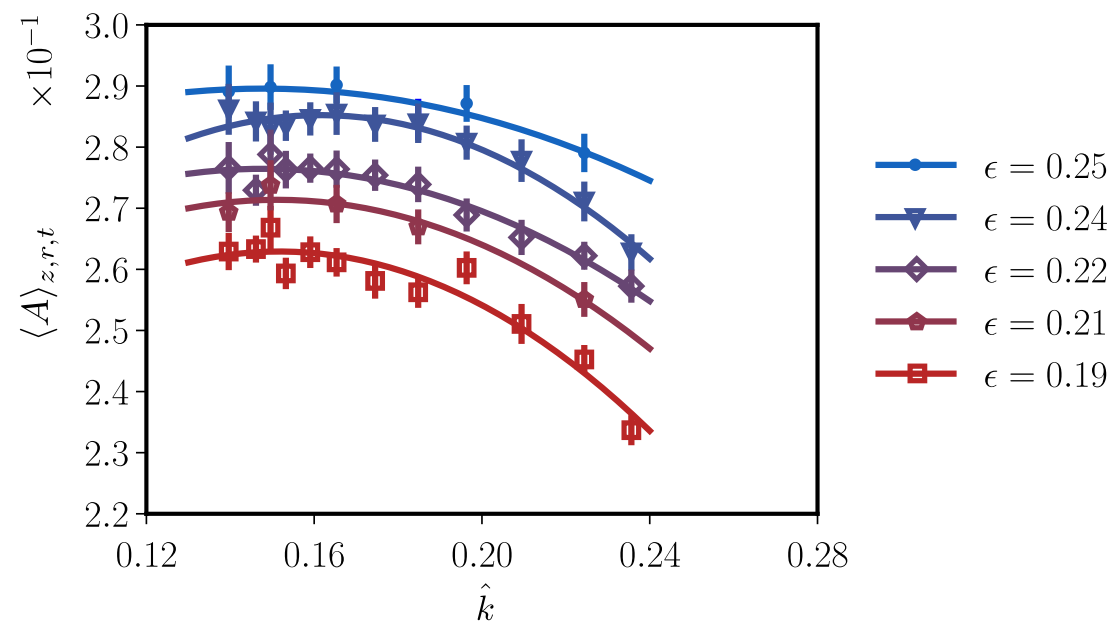

Figure 5.8: Amplitude $A$ of the finite wavelength instability versus wavenumber $\hat{k}$ for varying reduced thresholds $\epsilon=\left(R e_{i, c}-R e_{i}\right) / R e_{i, c}$. We include all simulations, with varying aspect ratios of $42 \leq \Gamma \leq 125$. Note that for large aspect ratios multiple spirals exist. Therefore we cannot simulate smaller wavenumbers than indicated in the graph. We observe consistent behaviour of the amplitude with increasing $\epsilon$, following the phenomenology of a finite wavelength instability. We fit a second order polynomial through the data. From this fit we obtain the most unstable wavelength for each $\epsilon$ - thus 5 values in total. We obtain $38.91 d<\lambda_{c}<42.52 d$ with mean $\left(\lambda_{c}\right)=41.38 d$ and $\operatorname{var}\left(\lambda_{c}\right)=1.74 d$. As we do not observe a systematic trend in the difference between the data and the fit, but rather find that the error is of similar order for all wavenumber $\hat{k}$, we conclude that a parabolic fit is justified. 
with increasing $\epsilon$. We conclude that the most unstable wavelength $\lambda_{z, c} / d \approx 41$ for our simulations at $\eta=0.91$ differs from the most unstable wavelength $\lambda_{z, c} / d \approx 32$ found in [17] at $\eta=0.98$.

In knowing that the amplitude in figure 8 is described by $A^{2}=\left(\epsilon-\xi_{0}^{2}(k-\right.$ $\left.\left.k_{c}\right)^{2}\right) / g_{0}$, we can also extract the interaction strength coefficient $g_{0}=\epsilon / \max _{k}\left(A^{2}\right)$ and the coherence length coefficient $\xi_{0}$ from the fits for varying $\epsilon$. We find that $g_{0}=2.80 \pm 0.11$ and does not depend on $\epsilon$, i.e. it does not depend on the magnitude of the noise as also found in [154]. The curvature we find at $k_{c}$ (i.e. $\left.\xi_{0}^{2} / g_{0} \approx 1.5\right)$ is comparable to the values observed by [17] (i.e. $\xi_{0}^{2} / g_{0} \approx 0.5$ ), but unfortunately our data are not precise enough to extract the coherence length $\xi_{0}$ of the pattern with sufficient accuracy to study the trends with $\epsilon$. It is interesting to note that in doing so, we are in principle able to extract properties of the full amplitude and pattern (like $\xi_{0}$ ) within the GL framework from an analysis of a single mode with fixed wavenumber (the Landau-Hopf or Stuart-Landau framework) induced by varying the aspect ratio, and that the resulting coherence length is much larger than our system size.

\subsection{Summary and conclusions}

In conclusion, we performed direct numerical simulations (DNSs) of counterrotating Taylor-Couette (TC) flow at $400 \leq R e_{i} \leq 1200$ and $-2000 \leq R e_{o} \leq$ -1000 . For the aspect ratio $\Gamma$ of the domain $42 \leq \Gamma=L / d \leq 125$ with periodic boundary conditions in the axial direction, and the radius ratio $\eta=$ $r_{i} / r_{o}=0.91$. In this regime we found the coexistence of spatio-temporal intermittent laminar and turbulent domains, commonly referred to as spiral turbulence $[16,141]$.

The formation of the turbulent structure (i.e. spiral in TC) out of a turbulent base flow is similar to the phenomenology of the (Complex) Ginzburg-Landau (GL) equations with a noise term [17], in the limit of very low curvature $(\eta=0.98)$. With these fully resolved simulations, we showed that the GL phenomenology also holds for the spirals when curvature effects do play a role. We found that the GL description for pattern formation holds close to the bifurcation point for both varying $R e_{i}$ and varying $R e_{o}$. This once more suggests the existence of a finite wavelength instability in a fully turbulent flow.

Also, we found that the pattern is stationary in the reference frame of the mean angular velocity in the domain $\omega_{s}=\langle\omega\rangle$. This is in contrast to findings by [157] and [138], who experimentally find that the spiral moves with the 
mean velocity of the two rotating cylinders, i.e. $\omega_{s}=\frac{1}{2}\left(\omega_{i}+\omega_{o}\right)$. However note, the small difference between $\langle\omega\rangle$ and $\frac{1}{2}\left(\omega_{i}+\omega_{o}\right)$ is hard to observe in experiments at these low Reynolds numbers.

In contrast with [17] we have periodic boundary conditions in the axial direction. As such, we only allow a wavelength which is an integer division of the aspect ratio $\Gamma$. It is therefore very likely that we do not observe a Reynolds number dependence of $\lambda_{z}$. In their experiment, [17] however employ no slip boundary conditions on the vertical axis, and as such allow for any wavelength to exist. Despite the different boundary conditions we find strong agreement in the critical Reynolds at which the instability occurs (i.e. $R e_{i, c}=863$ for our DNS and $R e_{i, c}=857$ for the experiments in [17]) as we also find similar values of the interaction strength $\xi_{0}^{2} \approx 1$ for both. Whereas we obtain $k_{c}$ by altering the aspect ratio, and [17] from analysis of monodomain regions in the flow, we both find a parabolic trend of the amplitude with $k$, where $k_{c}$ does not appear to depend on Re.

The most unstable wavelength of the instability is found to be $\lambda_{z, c} \approx 41 d$, thereby differing from findings in plane Couette flow and in TC flow, where it is $\lambda_{z, c} \approx 32 d$ at very high $\eta=0.98$. Apparently, $\lambda_{z, c}$ is a function of the radius ratio $\eta$. This finding may be an important clue for further theoretical investigations, and it may point the way towards understanding the modulated turbulent states in terms of a stationary bifurcation.

Whereas a formal derivation of the instability from the turbulent base flow seems hopeless, since it requires a closed description of the turbulence, fully resolved simulations help to uncover the characteristics and ingredients of the instability when it appears or disappears in the flow. Future work could continue on this road, by e.g. studying the response of the structure to abrupt changes in the boundary conditions, or by studying the effects of initial conditions on the turbulent spiral. 


\section{Chapter 6}

\section{The large-scale footprint in small-scale Rayleigh-Bénard turbulence}

Turbulent convection systems are known to give rise to prominent large scale circulation. At the same time, the 'background' (or 'small-scale') turbulence is also highly relevant and e.g. carries the majority of the heat transport in the bulk of the flow. Here, we investigate how the small-scale turbulence is interlinked with the large-scale flow organization of Rayleigh-Bénard convection. Our results are based on a numerical simulation at Rayleigh number $R a=10^{8}$ in a large aspect ratio $(\Gamma=32)$ cell to ensure a distinct scale separation. We extract local magnitudes and wavenumbers of small scale turbulence and find significant correlation of large scale variations in these quantities with the large-scale signal. Most notably, we find stronger temperature fluctuations and increased small scale transport (on the order of $10 \%$ of the global Nusselt number $N u$ ) in plume impacting regions and opposite trends in the plume emitting counterparts. This concerns wall distances up to $2 \delta_{\theta}$ (thermal boundary layer thickness). Local wavenumbers are generally found to be higher on the plume emitting side compared to the impacting one. A second independent approach by means of conditional averages confirmed these findings and yields additional insight into the large-scale variation of small-scale properties. Our results have implications for modelling small-scale turbulence.

In Press as: P. Berghout, W.J. Baars and D. Krug The large-scale footprint in small-scale Rayleigh-Bénard turbulence, in J. Fluid Mech. (2020). 


\subsection{Introduction}

Natural convection is an important flow configuration with highly relevant applications in thermal convection in the atmosphere [158] and in the stars [159] among many others. Fundamental aspects of natural convection are traditionally studied in the Rayleigh-Bénard set-up, in which the flow evolves between parallel horizontal plates that are heated from below and cooled from above $[112,160-162]$. In this case, the strength of the thermal driving is expressed by the dimensionless Rayleigh number $R a$, while, the dimensionless heat flux, which is the most important system response, is quantified by the Nusselt number $N u$.

A characteristic and very persistent feature of Rayleigh-Bénard convection (RBC) is the formation of a large-scale circulation (LSC). The LSC typically consists of one or several roll structures that fill the entire gap between the plates. Such structures continue to exist even in the highly turbulent state, at large $R a$. It has further been amply demonstrated e.g. [163-169] that the lateral extent of the LSC can reach multiple times the cell height $H$ in large aspect ratio domains. In laterally unconfined geometries, the LSC has therefore also been referred to as 'superstructure' in the more recent literature.

The existence of such large scale organization in an otherwise chaotic flow is arguably an exciting aspect of $\mathrm{RBC}$, and is reminiscent of large-scale structures in other canonical flows $[170,171]$. Their slow spatio-temporal evolution renders these structures prime candidates for flow modelling approaches. Such efforts are indeed already under way, e.g. by [172] who used deep learning techniques to analyse superstructures up to moderate $R a$ of $10^{7}$. However, these authors also acknowledged that the relative contribution of the LSC to the total heat transport decreases with increasing $R a$. Similarly, [169] found that at $R a=10^{8}$, superstructures account for a maximum of $30 \%$ of the total heat transport. It is therefore clear, that the 'background' turbulence (i.e. small to intermediate scales) plays an essential role that needs to be accounted for in low order models. This immediately raises the question on how structures of different sizes 'interact' in RBC or more precisely: How does a certain largescale state affect local properties of the small-scale field? This is exactly what is going to be addressed in this study.

Related findings on spatial variations in the small-scale statistics have recently been reported by [173]. These authors found that in the center regions of the LSC (i.e.in the wind-shear regions) the decay of temperature fluctuations away from the wall follows a power law dependence. Whereas in the plume emitting 
parts of the BL, temperature fluctuations drop of logarithmically with wall distance $z$. Relevant in the broader context are the studies by [174] and [175, 176], who derived a BL equation for the mean and variance of the temperature profile, where they included the effect of the fluctuations into the otherwise laminar 2D BL equation. Further, [177] disentangled temperature fluctuations throughout the RBC cell into homogeneous background (Gaussian) and nonhomogeneous plume contributions (exponential).

The approach we choose here is to identify and to quantify modulation effects between large and small scales in terms of amplitude and wavenumber. Naturally, this first and foremost necessitates a clear definition of what those 'large' (i.e. related to the LSC) and 'small' (the rest) scales are. Such a distinction can readily be made for a superstructure configuration (aspect ratio $\Gamma=32$ ) at $R a=10^{8}$, as figure 6.1 demonstrates. The premultiplied (multiplied with the wavenumber $k$ ) temperature spectrogram $k \Phi_{\theta \theta}$ shown there clearly separates into a large-scale peak at radial wavenumber $k \approx 1$ and one at significantly smaller scales $\left(k \sim 10^{2}\right)$. There is only little energy in the spectral gap between the two peaks, such that a scale decomposition can be achieved via spectral filtering. Here we chose a cut-off wavenumber $k_{\text {cut }}=2.5$ (indicated by the red line in the figure), but note that the results are rather insensitive to the exact choice of $k_{\text {cut }}$. The normalized wavelength $\hat{l}=2 \pi / \hat{k}$ where $\hat{k}$ is a characteristic wavenumber of the LSC based on a coherence metric, see [169] of the large scale structures increases with increasing $R a$ [167-169], while the structure size corresponding to the small scale peak scales with the thermal BL height $\delta_{\theta}[169]$. As demonstrated in [178], this leads to an increasing scale separation between the peaks at higher $R a$, rendering their interaction even more clear and relevant.

For the reasons outlined above, large aspect ratio cells are convenient for the present purpose and are therefore considered in the following. However, we note that there is little reason to believe that the effects studied are restricted to such domains. In fact, we expect scale interactions similar to those uncovered here to also play a role in smaller cells. Yet, spectral overlap of the peaks and other complications, e.g. due to corner vortices, render their analysis much more cumbersome in these cases.

Finally, we point out that a considerable number of studies on scale interactions in other flows exist, tracing back to [179] and [180]. Especially in the field of turbulent BLs, where a scale separation reminiscent of the situation displayed in figure 6.1 occurs [181], this topic has attracted considerable attention over the last decade [182-189]. We will make use of the mature frame- 


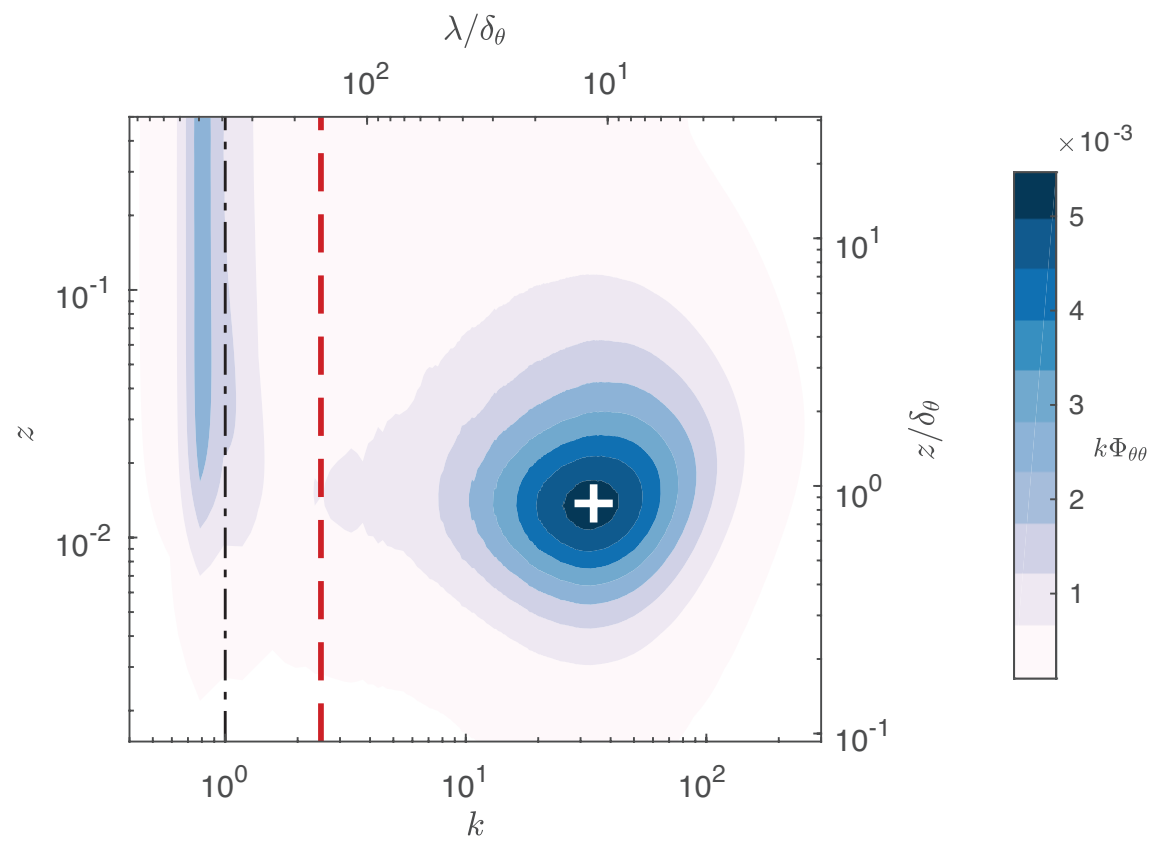

Figure 6.1: Premultiplied temperature spectrogram $k \Phi_{\theta \theta}$ at $R a=10^{8}$ and $\Gamma=32$, where $k$ is the wavenumber and $\lambda=(2 \pi) / k$ the wavelength. The white cross located at $z=0.85 \delta_{\theta}$ and $k=34\left(\lambda / \delta_{\theta} \approx 11.4\right)$ indicates the location of the small-scale or 'inner' peak. The black dashed-dotted line marks the superstructure scale $\hat{l}$ determined in [169] based on the coherence metric and the red dashed line is located at the cutoff wavenumber $k_{\text {cut }}=2.5$ used here. 


\begin{tabular}{ccc|ccccc}
$R a$ & $N_{x} \times N_{y} \times N_{z}$ & $\Gamma$ & $t / T$ & $N u$ & $\hat{l} / H$ & $\delta_{\theta} / H$ & $N_{\theta}$ \\
\hline $1.0 \times 10^{8}$ & $8192 \times 8192 \times 256$ & 32 & 200 & 30.94 & 6.3 & 0.016 & 13
\end{tabular}

Table 6.1: Parameters of the dataset. Input parameters are the $R a$ number, the numerical resolution in the horizontal $\left(N_{x} \times N_{y}\right)$ and wall normal $\left(N_{z}\right)$ directions, the aspect ratio $\Gamma$, an the runtime $t$ of the DNS normalized by the free-fall timescale $T$. Relevant results are the $N u$ number, the superstructure size $\hat{l}$ which is calculated from the coherence spectrum in [169], the thermal BL thickness $\delta_{\theta}$ divided by the height of the domain $H$ and finally, the number of grid points that are in the thermal BL $N_{\theta}$.

work and tools developed in this context to quantify amplitude modulation and wavenumber modulation effects in RBC.

The chapter is structured as follows; in section 6.2 we present details of the numerical data set along with relevant parameters. We then quantify modulation effects using two independent analysis tools. First, using a wavelet-based method (section 6.3.1-section 6.3.2) and then in section 6.3.3 on the basis of conditioned statistics. We conclude by discussing some physical implications of the results in section 6.4.

\subsection{Governing equations and parameters}

The simulations used here have previously been reported in [168] and we include some relevant details here for completeness. The incompressible Navier Stokes (NS) equations within the Boussinesq approximation are solved using a second-order finite difference scheme. The dimensionless equations are:

$$
\begin{gathered}
\frac{\partial \tilde{\mathbf{u}}}{\partial t}+\tilde{\mathbf{u}} \cdot \nabla \tilde{\mathbf{u}}=-\nabla \tilde{p}+\sqrt{\frac{P r}{R a}} \nabla^{2} \tilde{\mathbf{u}}+\tilde{\theta} \mathbf{e}_{z}, \\
\nabla \cdot \tilde{\mathbf{u}}=0, \\
\frac{\partial \tilde{\theta}}{\partial t}+\tilde{\mathbf{u}} \cdot \nabla \tilde{\theta}=\frac{1}{\sqrt{R a P r}} \nabla^{2} \tilde{\theta} .
\end{gathered}
$$

where the characteristic length scale is the height of the RBC cell $H$, the free-fall time scale $T=\sqrt{H /(g \alpha \Delta)}$ and the velocity scale is $\sqrt{g \alpha \Delta H}$, which is know as the free-fall velocity. Therein, $g$ is the gravitational acceleration (acting in opposite direction of the unit vector $\mathbf{e}_{z}$ ), $\alpha$ is the thermal expansion coefficient and $\Delta$ is the temperature difference between the top and the 
bottom plate. Two dimensionless parameters appear after normalizing the NS equations, namely the Prandtl number $\operatorname{Pr}=\nu / \kappa$ and the Rayleigh number $R a=\alpha g \Delta H^{3} /(\nu \kappa)$, where $\kappa$ is the thermal diffusivity and $\nu$ the momentum diffusivity (kinematic viscosity). Finally, the geometric control parameter is the aspect ratio $\Gamma=L / H$ of the square computational domain, with $L$ denoting the length of the cell in the periodic $x$ and $y$ directions. Instantaneous normalized values of pressure, temperature, and the velocity vector are denoted by $\tilde{p}, \tilde{\theta}$ and $\tilde{\mathbf{u}}$, respectively and we refer to the components of $\tilde{\mathbf{u}}$ as $\tilde{v}$ (horizontal) and $w$ (vertical). The operator $\langle\cdot\rangle$ is used to indicate averaging over the statistically homogeneous horizontal plane and time. We use the notation $\tilde{\xi}(x, y, z, t)=\Xi(z)+\xi(x, y, z, t)$ to decompose an arbitrary instantaneous quantity $\tilde{\xi}(x, y, z, t)$ into its mean $\Xi(z)=\langle\tilde{\xi}\rangle$ and a fluctuating part $\xi(x, y, z, t)$.

Parameters of the DNS are summarized in table 6.1. Importantly, the BLs are well resolved and comply with the criterion derived in [174].

\subsection{Results}

The essence of this study is best illustrated by the snapshot of the temperature field shown in figure 6.2a. There, the colour-contour represents $\theta_{S}\left(z=\delta_{\theta}\right)$, i.e. the small-scale temperature fluctuations at BL height. Note that we use subscripts $S, L$ to represent the small $\left(k>k_{\text {cut }}\right)$ and large-scale $\left(k<k_{\text {cut }}\right)$ signals, respectively. Even though the large-scales - and hence the effect of a direct superposition of the LCS onto fluctuations at BL height observed before [167-169] — have been removed, there is still a distinct large-scale 'organisation' visible for $\theta_{S}$. This pattern stems from spatial variations of properties such as the local amplitude and the local wavenumber of $\theta_{S}$. Figure 6.2a further contains zero-crossings of $\theta_{L}$ (dashed lines), which make it obvious that these spatial variations in the small-scale signal are highly correlated to the large-scale dynamics. In the next section we will introduce a procedure to capture the large-scale 'footprint' in small scale turbulence by extracting local amplitude and wavenumber. On this basis we can then quantify the correlation of this footprint with the large scales.

\subsubsection{Reduced-order representation of small-scale fluctuations}

To quantify how the large-scale RB superstructures interplay with the smallscale turbulence $\left(\xi_{S}\right)$, we have to reside to a simplified representation of the 

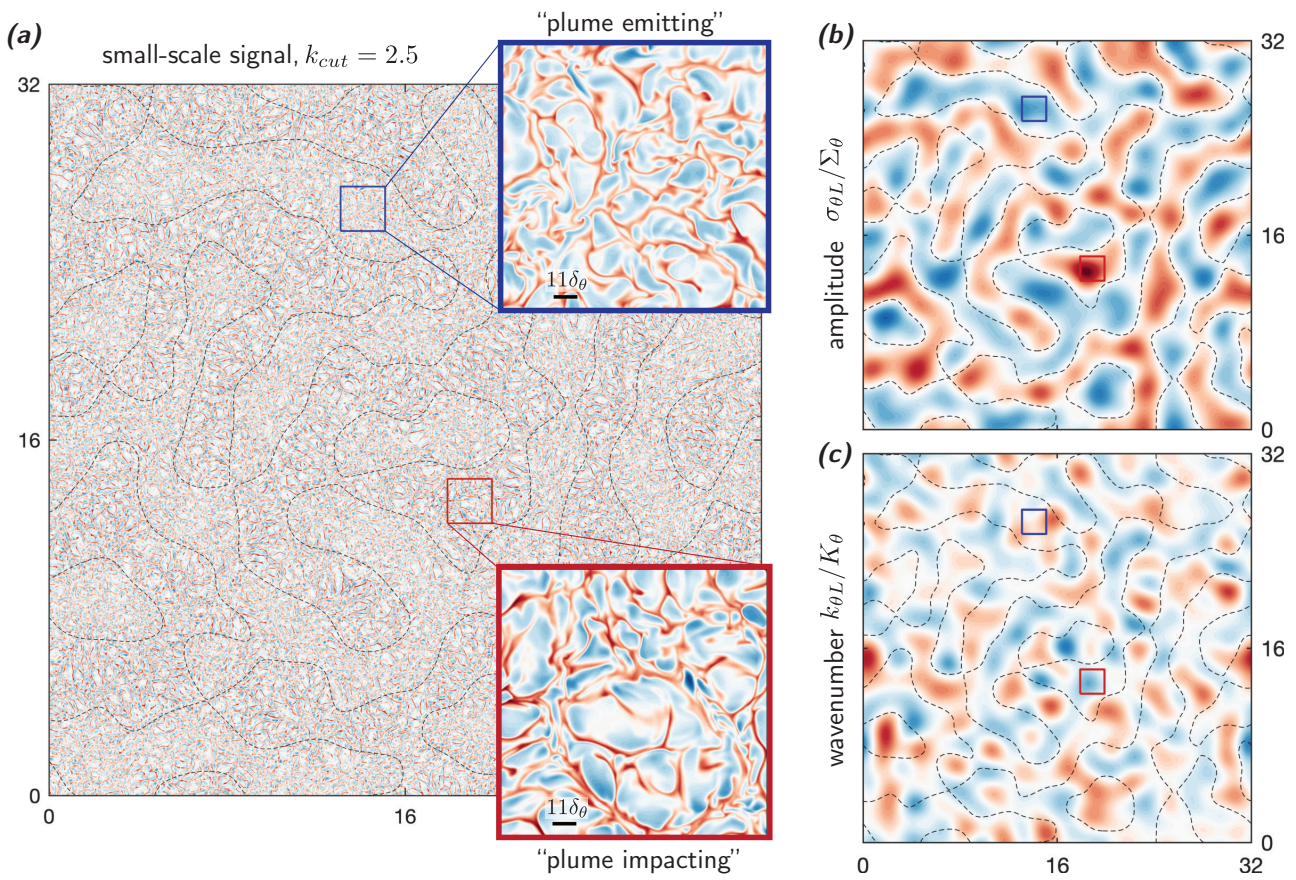

Figure 6.2: (a) Snapshot of the small-scale temperature fluctuations $\theta_{S}$ at a distance $z=\delta_{\theta}$ above the hot plate. Blow-ups show plume emitting $\left(\theta_{L}>0\right.$, blue box) and plume impacting $\left(\theta_{L}<0\right.$, red box) regions. Panels (b) and (c) show the large-scale amplitude $\sigma_{\theta L}$ and wavenumber $\kappa_{\theta L}$, respectively for the snapshot shown in (a). The boxes indicate the positions of the blow ups in (a). Dashed black lines in all panels correspond to zero-crossings in the large-scale signal $\theta_{L}$ at mid-height $z / H=0.5$. The scales of the color axes are \pm 0.36 , \pm 0.2 and \pm 0.4 in (a), (b) and (c), respectively. 
small-scale fluctuations ( $\xi$ is a placeholder for the quantity concerned, e.g. $\theta$, $w, \ldots$ ). These fluctuations are broadband e.g. [169] and pose a challenge to reduce the wealth of information in $\xi_{S}(x, y)$ to a comprehensible set of representative quantities. Here we apply an energy-based wavelet procedure [190], which preserves the large-scale spatial variation of the small-scale statistics in an $(x, y)$-plane. Our procedure yields two new variables: $(i)$ the space-varying magnitude $\sigma_{\xi}$ and (ii) the space-varying characteristic wavenumber $k_{\xi}$ of the energy in $\xi_{S}(x, y)$. Through correlating these two representative variables of the small-scale turbulence with the large-scale superstructures in $\xi_{L}$, we study the interaction between the two energetic regions that were identified in spectral space (see the discussion alongside figure 6.1).

Our procedure to obtain the reduced-order representation of the small-scale fluctuations is illustrated in figure 6.3. A wall-parallel plane of $\xi$ forms the starting point in this diagram (top-left of figure $6.3 b$ ). For ease of visualization, a one-dimensional subset taken along the $x$-direction of $\xi$ is shown in figure 6.3( $a)$ for one particular $y=y_{p}$ location. Inspection yields a large-scale variation of the fluctuating magnitude $\sigma_{\xi L}$ (indicated by the dashed envelope) and a spatial variation in the characteristic wavenumber $k_{\xi L}$; this reflects the spatial variation in small scale properties already observed in figure 6.2a.

The first step in quantifying the large-scale space-varying characteristics of the small-scale turbulence involves a two-dimensional (2D) continuous wavelet transform (CWT). We restrict the discussion of the wavelet transform to a minimum here and refer to e.g. [191] and [192] for further mathematical intricacies. A mother wavelet $\Psi$ is convoluted with $\xi(x, y)$, following

$$
A_{\xi}\left(x, y ; l_{s}\right)=\iint \xi\left(x^{*}, y^{*}\right) \Psi\left(\frac{x-x^{*}}{l_{s}}, \frac{y-y^{*}}{l_{s}}\right) \mathrm{d} x^{*} \mathrm{~d} y^{*},
$$

where $A$ is the wavelet coefficient at wavelet scale $l_{s}$. The convolution is performed in the wavenumber domain and is repeated for a range of spatial wavelet scales $l_{s}$, in order to decompose $\xi(x, y)$ in space-scale space. The wavelet is considered a localized basis function (as opposed to harmonic basis functions in a Fourier analysis) and the mother wavelet used is the isotropic Morlet wavelet, which discards any angular sensitivity (the wavelet is symmetric). This is a suitable choice for RB turbulence, as there is no angular dependence of the statistics in this flow. Alternative choices (such as the Mexican hat wavelet) with different resolution characteristics in wavenumber vs. physical space were found to not affect the conclusions made throughout the manuscript. Further mathematical intricacies of the wavelet transform can be found elsewhere e.g. [191,192]. 
(a)
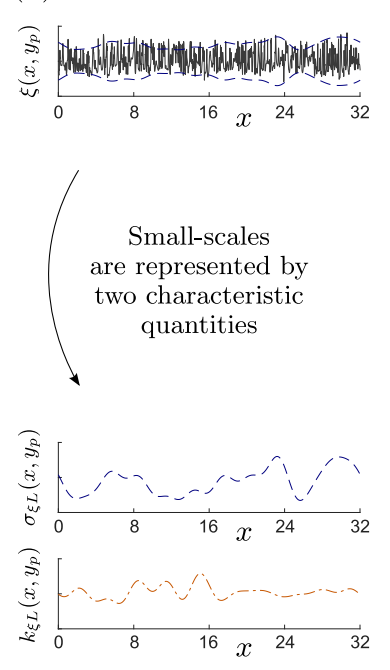

(b)

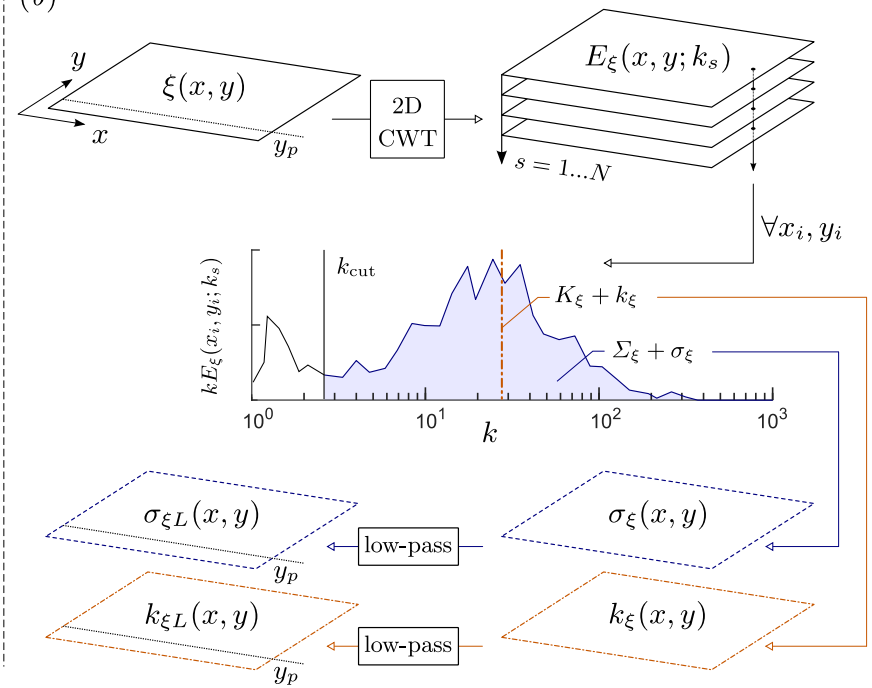

Figure 6.3: Diagram of the wavelet-based procedure, used in extracting local fluctuation properties of a turbulent quantity in a plane of RB turbulence, in particular the characteristic energy-amplitude of the fluctuations, $\sigma_{\xi L}(x, y)$, and a characteristic wavenumber of the fluctuations, $k_{\xi L}(x, y)$.

Scale argument $l_{s}$ can be transformed to a pseudo-wavenumber $k_{s}$, so that coefficients $A_{\xi}\left(x, y ; k_{s}\right)$ reflect the convolution magnitude as a function of the characteristic wavenumber of the wavelet. With the proper normalization, the square of the wavelet coefficients measures the energy level of a given field in terms of space and scale, yielding the energy density

$$
E_{\xi}\left(x, y ; k_{s}\right)=\frac{A_{\xi}\left(x, y ; l_{s}\right)^{2}}{l_{s}}
$$

The stack of $N$ planes in the top-right of figure $6.3(b)$ represents quantity $E_{\xi}\left(x, y ; k_{s}\right)$ computed at $N$ different scales. For a given sample location, denoted as $\left(x_{i}, y_{i}\right)$, the local wavelet spectrum $E_{\xi}\left(x_{i}, y_{i} ; k_{s}\right)$ is shown as a function of $k$. A total of $N=33$ logarithmically spaced scales were used in the smallscale range $k_{\text {cut }}<k<k_{N}$, with $k_{N}=4.5 \times 10^{3}$ denoting the DNS Nyquist wavenumber. The local wavelet spectrum is a measure of the energy spectrum at one spatial location. It is influenced by a domain surrounding $\left(x_{i}, y_{i}\right)$, which is referred to as the cone-of-influence of the wavelet, i.e. the region 
spanned by the wavelet at a certain scale: a small-scale wavelet is more localized than a larger-scale wavelet. Since the boundaries of the computational domain are periodic, localized wavelet spectra exist for all points in the $(x, y)$ domain. When all localized spectra are averaged, one global wavelet spectrum is found for the full $(x, y)$ field which then matches the Fourier spectrum at one $z$ height in figure 6.1 . So, the key feature of $E_{\xi}\left(x, y ; k_{s}\right)$ is that it preserves spatial information, which is absent in Fourier spectra.

So far, the decomposition of $\xi(x, y)$ into energy densities $E_{\xi}\left(x, y ; k_{s}\right)$ at $N$ different scales has increased the dimensions of the system. For a reducedorder representation of the small-scale turbulence, we now define the following quantities: First we introduce a spatial field that captures the variation in the energy magnitude of $\xi(x, y)$. Integrating $E_{\xi}\left(x, y ; k_{s}\right)$ over the small-scale wavenumber range $k_{\text {cut }}<k<k_{N}$ and taking the square root yields the spacevarying standard deviation of $\xi_{S}(x, y)$ according to

$$
\Sigma_{\xi}+\sigma_{\xi}(x, y)=\left(\int_{k_{\text {cut }}}^{k_{N}} E_{\xi}\left(x, y ; k_{s}\right) \mathrm{d} k_{s}\right)^{0.5} .
$$

Here, $\Sigma_{\xi}$ denotes the mean standard deviation of the small-scale fluctuations (i.e. $\Sigma_{\xi}=\left\langle\xi_{S}^{2}\right\rangle^{\frac{1}{2}}$ ), whereas $\sigma_{\xi}(x, y)$ is the zero-mean spatial fluctuation of the standard deviation. Only the large-scale variation of $\sigma_{\xi}$ is retained by lowwavenumber pass-filtering with $k<k_{\text {cut }}$. The resulting spatial field $\sigma_{\xi L}(x, y)$ is interpreted as an envelope to the small-scale fluctuations and is plotted in figure 6.3(a). A second representative property of $\xi(x, y)$ is its local wavenumber, which we take as the first spectral moment of the local wavelet spectrum,

$$
K_{\xi}+k_{\xi}(x, y)=\frac{\int_{k_{\text {cut }}}^{k_{N}} k E_{\xi}\left(x, y ; k_{s}\right) \mathrm{d} k_{s}}{\int_{k_{\text {cut }}}^{k_{N}} E_{\xi}\left(x, y ; k_{s}\right) \mathrm{d} k_{s}}=\frac{1}{\left(\Sigma_{\xi}+\sigma_{\xi}(x, y)\right)^{2}} \int_{k_{\text {cut }}}^{k_{N}} k E_{\xi}\left(x, y ; k_{s}\right) \mathrm{d} k_{s} .
$$

Similarly as for the standard deviation, the mean wavenumber of the smallscale energy in $\xi(x, y)$ is $K_{\xi}$ and its zero-mean fluctuations are denoted as $k_{\xi}(x, y)$. Low-wavenumber pass-filtering the latter yields $k_{\xi L}(x, y)$. In summary, the raw small-scale field $\xi_{S}(x, y)$ is represented by $\sigma_{\xi L}(x, y)$, which reflects the large-scale spatial deviation of the energy (or intensity) in the smallscale fluctuations from its mean energy. Further $k_{\xi L}(x, y)$ describes the deviation of characteristic local wavenumber from the mean wavenumber. Thus, positive (negative) $k_{\xi L}$ means that the small-scale fluctuations are of a higher (lower) wavenumber locally, compared to an averaged over the entire domain. Results of $\sigma_{\theta L}$ and $k_{\theta L}$ computed for the snapshot in figure 6.2a are included 
in figure $6.2 \mathrm{~b}, \mathrm{c}$, respectively. To what degree these quantities are correlated with the large-scale signal can be gauged by comparing to the zero-crossings of $\theta_{L}$ (shown as black lines). We will quantify this aspect next.

\subsubsection{Correlations of large and small scales}

To study the influence of the large scales on the small scales in RBC, we first define the amplitude modulation coefficient. This coefficient is the normalized correlation between the large-scale envelope of the small-scale turbulence $\sigma_{\xi, L}(x, y, z)$ and the large-scale signal of the temperature at mid-height $\theta_{L}(x, y, H / 2)$,

$$
R_{\xi, \sigma}(z)=\frac{\left\langle\sigma_{\xi L}(x, y, z) \theta_{L}(x, y, H / 2)\right\rangle}{\sqrt{\left\langle\sigma_{\xi L}(x, y, z)^{2}\right\rangle} \sqrt{\left\langle\theta_{L}(x, y, H / 2)^{2}\right\rangle}} .
$$

It is important to emphasize that in this definition, we always use the largescale temperature signal at mid-height $\theta_{L}(x, y, H / 2)$ as reference. Clearly, other choices are possible here. However, since the LSC is known to be highly coherent along the vertical direction and because $\theta_{L}$ correlates almost perfectly with the large-scale velocity signal [169], this will have no significant impact on our results and conclusions. By definition, $R_{\xi, \sigma}$ varies between -1 (perfect anti-correlation) and 1 (perfect correlation and $R_{\xi, \sigma}=0$ implies uncorrelated signals.

Analogously, the wavenumber modulation coefficient $R_{k, \xi}(z)$ is defined by correlating the large-scale part of the wavenumber fluctuations $k_{\xi L}(x, y, z)$ to $\theta_{L}(x, y, H / 2)$,

$$
R_{\xi, k}(z)=\frac{\left\langle k_{\xi L}(x, y, z) \theta_{L}(x, y, H / 2)\right\rangle}{\sqrt{\left\langle k_{\xi L}(x, y, z)^{2}\right\rangle} \sqrt{\left\langle\theta_{L}(x, y, H / 2)^{2}\right\rangle}} .
$$

We note that (6.8) and (6.9) define a normalized correlation. The physical relevance of such a correlation, however, also depends on how much (absolute) energy is contained in the fields of $\sigma_{\xi, L}$ and $k_{\xi L}$. For the snapshot in figure 6.2 , fluctuations in both $\sigma_{\theta, L}$ and $k_{\theta L}$ are on the same order (tens of percent) as their respective means and hence significant. We will revisit this point in section 6.3.3 in more detail.

Results for both modulation coefficients in the temperature field are presented as a function of the height $z$ in figure 6.4a. The most striking observation from this plot is that the small-scale amplitude of $\theta$ is highly correlated to the 

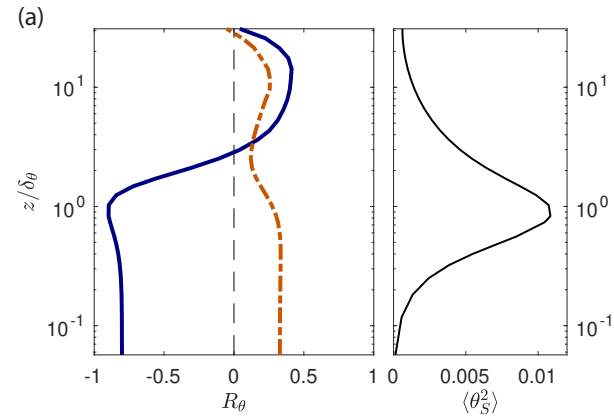

(c)

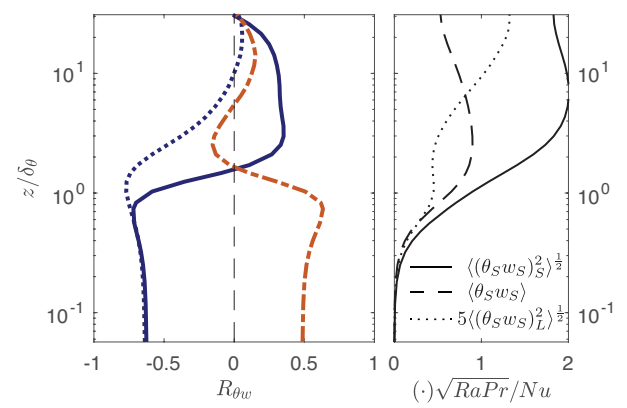

(b)

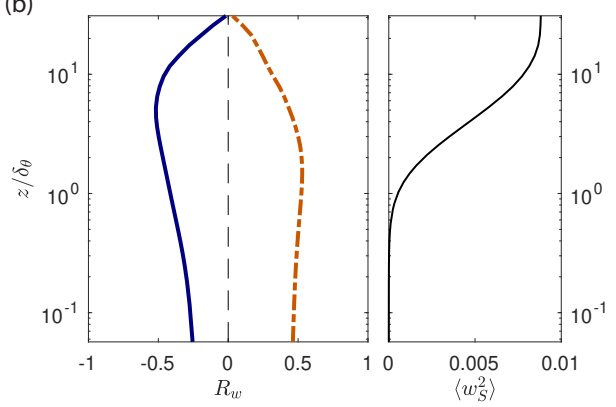

(d)

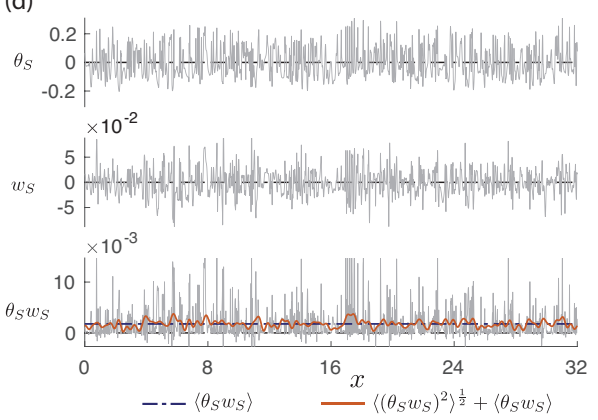

Figure 6.4: Wall-normal profiles of the amplitude modulation coefficient (solid dark blue), the wavenumber modulation coefficient (dash-dotted orange) and the small-scale energy $\bar{\sigma}_{\psi}^{2}$ (solid black) for (a) the temperature $\theta$, (b) the wall normal velocity $w$ and (c) the small-scale turbulent heat transport term $\left(\theta_{S} w_{S}\right)$. The blue dotted line in (c) is the correlation coefficient for $\left(\theta_{S} w_{S}\right)_{L}$. Panel (d) illustrates the construction of the $\left(\theta_{S} w_{S}\right)$ signal. It contains a largescale part (solid red), a non zero-mean (dashed black line) and the remaining small-scale signal, of which the respective variations with $z$ are shown in the right plot of panel (c). 
large scales up to a distance of about $2 \delta_{\theta}$ above the plate. The peak of this correlation at which remarkably $R_{\theta, \sigma}<-0.9$ is found at $z \approx \delta_{\theta}$. This coincides with the location of the strongest small-scale fluctuations in the temperature field as is obvious from the right-hand panel of figure 6.4(a) as well as from the spectral peak of the energetic small-scales in figure 6.1. Throughout this chapter, all statistics are plotted with reference to the hot plate. Hence, the negative sign of $R_{\sigma, \theta}$ in the near-wall region implies that in plume impacting regions (where $\left.\theta_{L}<0\right)$ the local small-scale energy is higher $\left(\sigma_{\theta L}>0\right)$. This is also confirmed visually from the blow-ups in figure 6.2a.

Interestingly, $R_{\theta, \sigma}$ crosses zero around $z=2 \delta_{\theta}$ and attains somewhat lower, yet distinctly non-zero values around $R_{\theta, \sigma} \approx 0.4$ in the bulk. The subsequent drop to zero at mid-height is a symmetry requirement. The crossover height of $z=2 \delta_{\theta}$ is consistent with recent findings by [173], who found similar levels of temperature fluctuations in plume emitting and sheared regions at this height. Further away from the wall, [173] found that plume emitting regions contain significantly higher fluctuations of $\theta$, which is also consistent with the present observations.

Compared to $R_{\theta, \sigma}$, the wavenumber modulation coefficient for $\theta$ (dashed red line) remains relatively low at all $z$. Also in this case, the strongest correlation encountered within the thermal BL with $R_{\theta, k} \approx 0.3$. Unlike $R_{\theta, \sigma}, R_{\theta, k}$ does not change sign and remains positive throughout, which implies that structures in the temperature field are slightly more 'compact' in plume emitting regions.

In principle, correlation coefficients can be evaluated for all fluctuating quantities. Here we will in addition to $\theta$ only consider the wall-normal velocity $w$. This is motivated by the fact that $w$ also features in the turbulent heat transport $\theta w$. From figure 6.4(b), we find that the amplitude modulation coefficient for $w$ is negative at all heights at intermediate correlation levels of about 0.4. On the contrary, $R_{w, k}$ is positive at all $z$ locations and reaches values up to 0.5 . Both, $R_{w, \sigma}$ and $R_{w, k}$ peak in the range 1-10 $\delta_{\theta}$, over which also the fluctuation level in $w_{S}$ builds up to its bulk value (see right-hand panel in figure 6.4b). The picture that emerges here is that $w_{S}$ fluctuations are of lower magnitude but higher wavenumber in plume emitting regions.

Besides the individual fields of $w_{S}$ and $\theta_{S}$, it is meaningful to study the modulation effects for the turbulent heat transport $\theta w$. Initially, we apply the same analysis leading to (6.8) and (6.9) also to $\theta w$. The corresponding results, $R_{\theta w, \sigma}$ (solid blue line) and $R_{\theta w, k}$ (dashed red), characterize zero-mean fluctuations of $(\theta w)_{S}$ and are shown in figure 6.4c. Both display substantial correlation with the large scales up to BL height, at which both the mean and the fluctuations 
in the normalised small-scale heat transport already reach the level of $N u$, as can be seen from the right panel in $6.4 \mathrm{c}$. Consistent with observations for $\theta$ and $w$ individually, we find $R_{\theta w, \sigma}<0$ and $R_{\theta w, k}>0$ for $z \lesssim \delta_{\theta}$, while at larger wall distances $R_{\theta w, \sigma}$ is slightly positive with no significant wavenumber modulation.

There is, however, another point to be made here. This relates to the fact that even though individually $\theta_{S}$ and $w_{S}$ are zero-mean and small-scale only, their product $\theta_{S} w_{S}$ contains not only a significant mean (that contributes the bulk of the total heat transport above $z \approx \delta_{\theta}$, see figure $6.4 \mathrm{c}$, right panel), but also large scale fluctuations. This is illustrated for a 1-D sample in figure $6.4 \mathrm{~d}$. The large scale variations $\left(\theta_{S} w_{S}\right)_{L}$ are not equally strong as those at smaller scales (note the factor 5 for the rms of $\left(\theta_{S} w_{S}\right)_{L}$ in figure $6.4 \mathrm{c}$ ), yet their standard deviation still amounts to about $10 \%$ of $N u$ around BL height which is significant. At this wall distance, $\left(\theta_{S} w_{S}\right)_{L}$ displays a pronounced negative correlation with $\theta_{L}$ (dotted blue line in figure 6.4c), which extends even somewhat deeper into the bulk compared to $R_{\theta w, \sigma}$. This implies that the local heat transport carried by small-scale fluctuations is locally reduced (increased) in plume emitting (impacting) regions around BL height.

Correlation coefficients are very useful in quantifying the overall nature of scale interactions. However, they do not reveal all details about the spatial organisation in the flow. We will therefore complement the results discussed so far by additionally considering conditioned averages in the following.

\subsubsection{Conditioned averages}

\section{Averaging procedure}

The results obtained so far indicate that the properties of the small-scale turbulence, especially in the vicinity of the BLs, vary significantly depending on the location within the LSC. In this section, we aim to investigate those spatial variations in more detail. To that end, we employ conditioned averages with respect to the zero crossing of the large-scale signal $\theta_{L}$. The definition of the new conditioned coordinate $d$ is illustrated in figure 6.5(a). For every point $P$ in a plane $d^{*}$ measures the smallest distance to a contour of $\theta_{L}=0$, i.e. $d^{*}$ is the smallest radius of a circle around $P$ that just touches a point on the zero-contour. To distinguish large-scale positive from large-scale negative regions, $d$ then takes the sign of $\theta_{L}$, such that

$$
d=\operatorname{sign}\left(\theta_{L}\right) d^{*} .
$$



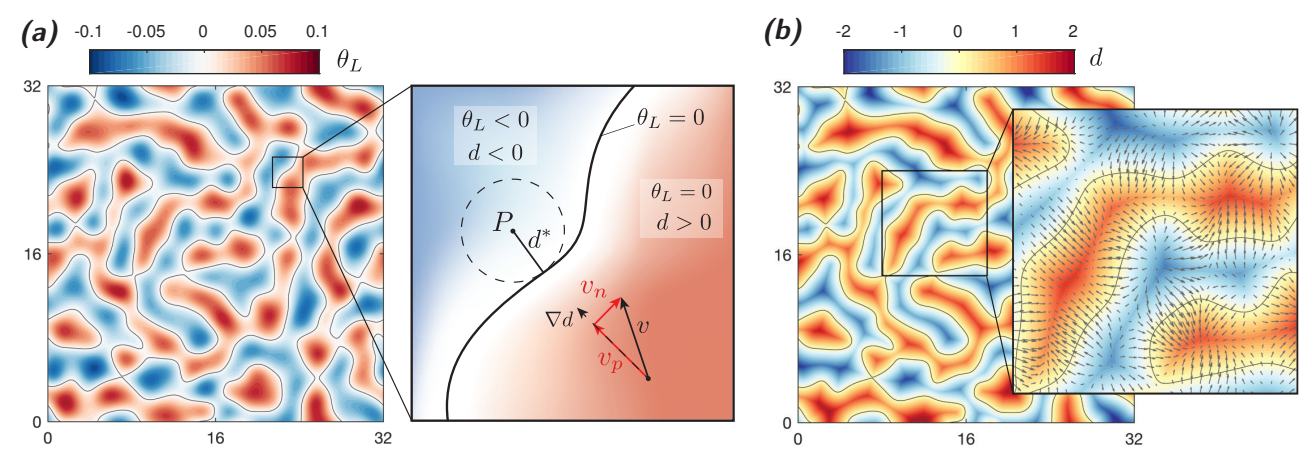

Figure 6.5: Illustration of the definition of the conditioned average. (a) Largescale part of the temperature fluctuations at mid-height $\theta_{L}(z / H=0.5)$. Black lines in all panels are iso-contours of $\theta_{L}=0$. Every point $P$ in the plane is assigned a distance $d$, where the magnitude of $d$ is determined by the distance to the nearest point on the iso-contour (see blow-up in (a)) and the sign is determined by that of $\theta_{L}$ at point $P$. The resulting $d$-field corresponding to the snapshot of $\theta_{L}$ in (a) is shown in panel (b). The in-plane velocity vector at each point (see blow-up in (b) is decomposed into a component along $\left(v_{p}\right)$ and normal $\left(v_{n}\right)$ to the gradient $\nabla d$ as shown in the blow-up in (a). 

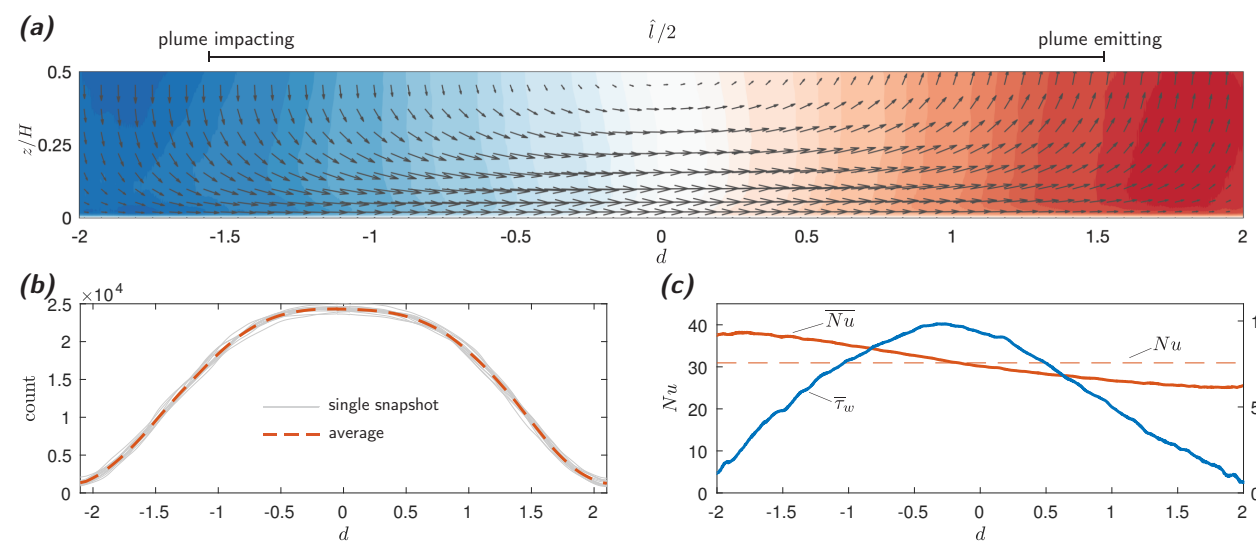

(c)

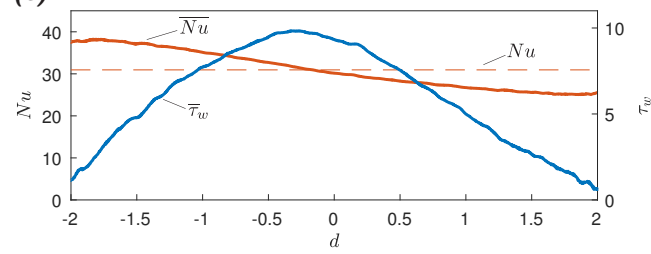

Figure 6.6: (a) Superstructure convection roll as obtained from the conditional averaging procedure. The color contours represent $\bar{\theta}$ and span the range \pm 0.1 . Vectors indicate the field $\left(\bar{v}_{p}, \bar{w}\right)$ with 15 data points skipped between adjacent vectors. (b) Number of points in a single plane as a function of the conditioning parameter $d$ for a total of 13 snapshots. (c) Variation of the conditioned Nusselt number $\overline{N u}$ and of the conditioned shear stress $\bar{\tau}_{w}$ as functions of $d$.

The $d$-field corresponding to the $\theta_{L}$ snapshot in figure 6.5(a) is shown in panel (b) of the same figure. The blow-up in figure $6.5 \mathrm{~b}$ presents the large-scale horizontal velocity vector at BL height (with the same cut-off wavenumber as $\left.\theta_{L}\right)$. This plot demonstrates that the LSC flow at the hot plate is generally directed from small $d$ to large $d$. The largest large-scale velocity magnitudes are encountered around $d \approx 0$. Since the large scale circulation is approximately aligned with $\nabla d$, it is useful to decompose the horizontal velocity vector $\mathbf{v}$ into a component $v_{p}$ along $\nabla d$ and one $\left(v_{n}\right)$ normal to it as illustrated in the blow-up of figure 6.5 a.

To retain the large-scale organization in the flow, we then compute conditional averages by taking the mean overall points with constant $d$. Mathematically, this results in a triple decomposition for any instantaneous quantity $\tilde{\xi}(x, y, z, t)$ according to

$$
\tilde{\xi}(x, y, z, t)=\Xi(z)+\bar{\xi}(z, d)+\xi^{\prime}(x, y, z, t),
$$

where the overline denotes the conditional average, and $\xi^{\prime}(x, y, z, t)$ is the fluctuation about the conditional and the temporal average.

Conceptually, the conditioned means $\bar{\xi}(z, d)$ correspond to the large-scale fluctuation field and represent the LSC, while the fluctuations $\xi^{\prime}(x, y, z, t)$ around 
the conditioned mean are related to small-scale turbulence. It is hence of interest to analyse the spatial distribution of statistics related to $\xi^{\prime}$ in the present context. Before doing so, we briefly present some results for the conditioned mean in order to orient the reader about basic properties of the LSC in the present configuration. For that purpose, the contour of the conditionally averaged temperature $\bar{\theta}$ is shown in figure $6.6(\mathrm{a})$ overlaid with vectors representing $\left(\bar{v}_{p}, \bar{w}\right)$. It is clear from this figure that the ranges $d<-1$ and $d>1$ correspond to the plume impacting and emitting zones of the LSC, respectively, and the strongest horizontal flow is encountered for $-1<d<1$. Further, the histogram in figure 6.6(b) shows that the distribution of $d$ is already well-converged from a single snapshot only with only few counts beyond half the typical superstructure size $\hat{l} / H \approx 3$ for this case. Relevant transport quantities that can be derived from the fields shown in figure 6.6(a) are the local wall shear stress $\bar{\tau}_{w}=\left|\left(\left.\partial_{z} \overline{v_{p}}\right|_{z=0}\right)\right|$, which is seen to peak at slightly negative $d$ (see figure 6.6c). Moreover, we observe a significant spatial variation of $\pm 0.3 N u$ for the local heat transport $\overline{N u}=\left.\partial_{z}(\Theta+\bar{\theta})\right|_{z=0}$. As figure 6.6c shows, heat transport is enhanced in plume impacting regions and decreased in plume emitting regions. These observations are similar to those reported for RBC at $\Gamma=1, \operatorname{Pr}=0.79$ and matching $R a$, see [193]. For a more detailed study on large-scale properties including their $R a$ dependence, we refer to $[178]$.

\section{Conditioned variance}

Several statistical properties are relevant when examining the fields of conditioned fluctuations $\xi^{\prime}$ in terms of their magnitude. The most basic one is the variance $\left\langle\xi^{\prime 2}\right\rangle(z)$, which indicates how energetic the small scales are at a given height (note that $\langle\cdot\rangle$ continues to imply horizontal averaging, in this case over $d)$. The small-scale temperature variance $\left\langle\overline{\theta^{\prime 2}}\right\rangle(z)$ is shown in $6.7 \mathrm{a}$ and indeed resembles $\left\langle\theta_{S}^{2}\right\rangle$ in figure 6.4a closely. The main interest here is in lateral (along $d)$ variations of the variance, which we highlight by considering the difference $\Delta \overline{\xi^{\prime 2}}(z, d)=\overline{\xi^{\prime 2}}(z, d)-\left\langle\overline{\xi^{\prime 2}}\right\rangle(z)$. This quantity is akin to the large scale envelope $\sigma_{\theta L}$. We plot the normalized standard deviation of $\Delta \overline{\theta^{\prime 2}}$ in figure $6.7 \mathrm{c}$ as a measure for how much the temperature variance varies with $d$ at a given height. Finally, we define the coefficient

$$
\rho_{\xi}(z, d) \equiv \frac{\overline{\xi^{\prime 2}}(z, d)-\left\langle\overline{\xi^{\prime 2}}\right\rangle(z)}{\left\langle\left(\overline{\xi^{\prime 2}}(z, d)-\left\langle\overline{\xi^{\prime 2}}\right\rangle(z)\right)^{2}\right\rangle^{1 / 2}}=\frac{\Delta \overline{\xi^{\prime 2}}(z, d)}{\operatorname{std}\left(\Delta \overline{\xi^{\prime 2}}\right)(z)},
$$



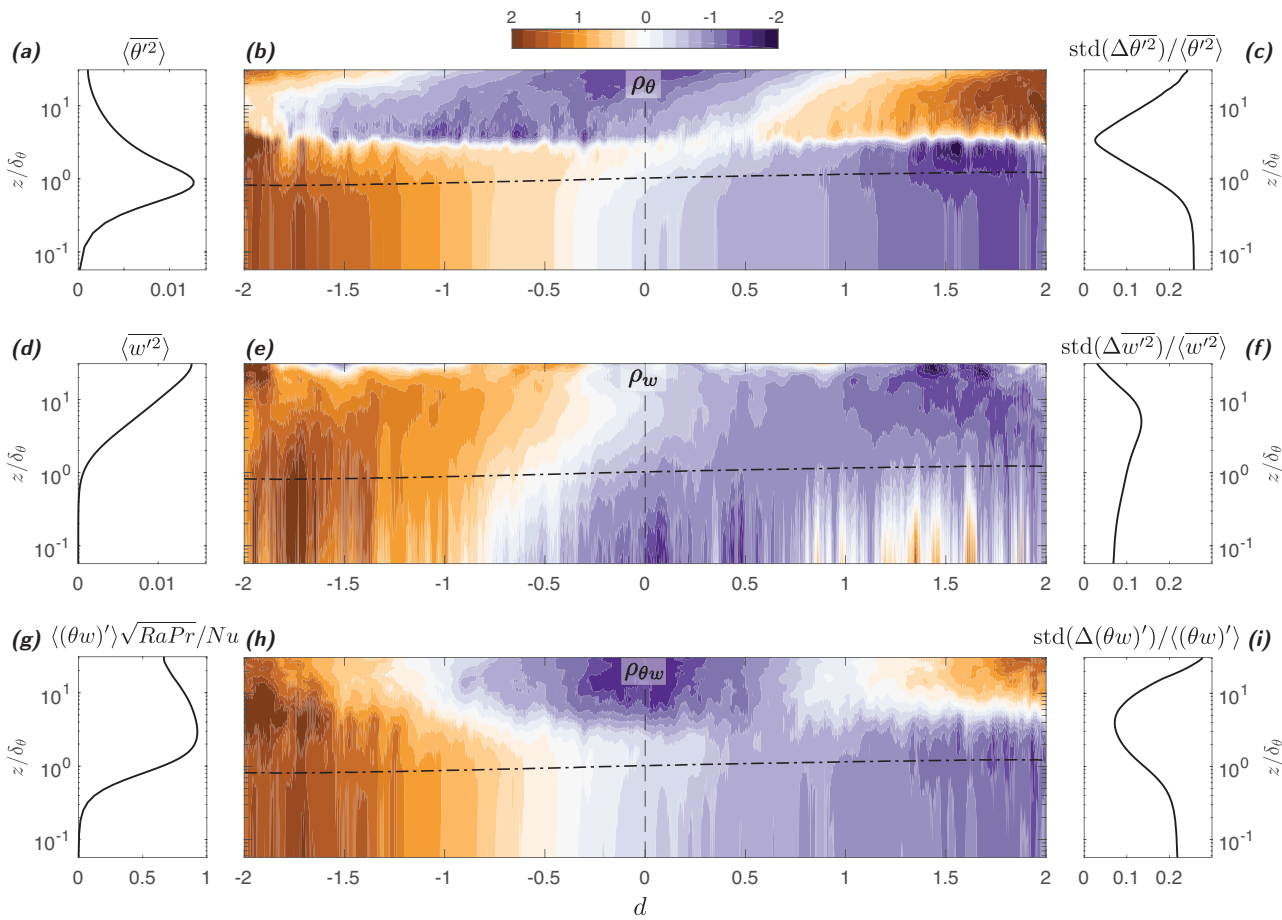

$\operatorname{std}\left(\Delta(\theta w)^{\prime}\right) /\left\langle(\theta w)^{\prime}\right\rangle$ (i)

Figure 6.7: Statistics of the conditioned fluctuations of $\theta$ (a-c) and $w$ (df). Additionally, statistics of $(\theta w)^{\prime}$ are shown in panels (g-i). Note that the standard deviation operator used in $(\mathrm{c}, \mathrm{f}, \mathrm{i})$ implies horizontal averaging only, i.e. $\operatorname{std}(\cdot)=\left\langle(\cdot)^{2}\right\rangle^{1 / 2}$. The horizontal dashed line in (b,e,h) shows the local thermal BL height $\bar{\delta}_{\theta}(d)=1 /(2 \overline{N u})$. The colorbar applies to all contour plots.

to illustrate how $\Delta \overline{\xi^{\prime 2}}$ varies across the LSC. Results for $\rho_{\theta}$ are displayed in figure $6.7 \mathrm{~b}$. Entirely consistent with figure 6.4(a), also this plot indicates enhanced (reduced) small-scale temperature fluctuations in the impacting (emitting) region below $z \approx 2 \delta_{\theta}$. At this height, both $\left\langle\overline{\theta^{\prime 2}}\right\rangle$ and $\Delta \overline{\theta^{\prime 2}}$ are of significant magnitude. Also the change in sign for $R_{\theta \sigma}$ towards positive values observed for $z \gtrsim 2 \delta_{\theta}$ is echoed in the distribution of $\rho_{\theta}$. Generally, $\rho_{\theta}>0$ at $d>0$ (and vice versa) in the bulk. However, the zero-crossing clearly does not coincide with $d=0$ in this case, which is testament to the additional information provided by considering the conditioned averages.

Results analogous to those presented for the temperature field in figure 6.4a-c 
are included for $w$ in panels d-f of the same figure. Also in this case, there is a striking agreement between the distribution of $\rho_{w}$, which indicates stronger fluctuations on the plume impacting side, and $R_{w \sigma}$ in figure $6.4 \mathrm{~b}$.

Finally, we turn again to the turbulent heat transport. Here, we define the small-scale contribution according to

$$
(\theta w)^{\prime}(z, d)=\overline{\theta w}(z, d)-\bar{\theta} \bar{w}(z, d),
$$

that is, we subtract the part of the heat transport carried by the LSC from the overall turbulent transport. As figure $6.7 \mathrm{~g}$ shows, $\left\langle(\theta w)^{\prime}\right\rangle$ carries a bulk of the global transport at wall distances beyond the thermal BL height (note also the agreement with $\left\langle\theta_{S} w_{S}\right\rangle$ in figure 6.4c). The corresponding coefficient

$$
\rho_{\theta w}(z, d) \equiv \frac{(\theta w)^{\prime}(z, d)-\left\langle(\theta w)^{\prime}\right\rangle(z)}{\left\langle\left((\theta w)^{\prime}(z, d)-\left\langle(\theta w)^{\prime}\right\rangle_{d}(z)\right)^{2}\right\rangle^{1 / 2}}=\frac{\Delta(\theta w)^{\prime}(z, d)}{\operatorname{std}\left((\theta w)^{\prime}\right)(z)},
$$

is analogous to (6.12) but provided explicitly for clarity. Importantly, it is clear from (6.13) and (6.14) that $(\theta w)^{\prime}$ and hence also $\rho_{\theta w}$ describe how the net heat transport by the small scales varies spatially. The results presented here therefore approximately correspond to $\left(\theta_{S} w_{S}\right)_{L}$ in figure $6.4 \mathrm{c}$. The contour plot of $\rho_{\theta w}$ (figure $6.7 \mathrm{~h}$ ) then also confirms the observation that up to several BL heights, small-scale heat transport is stronger in plume impacting regions. In the bulk, the correlation coefficient for $\left(\theta_{S} w_{S}\right)_{L}$ is essentially zero (see dotted blue line in figure 6.4c). However, this does not imply that the small scale heat transport is uniform there: As figure 6.7h reveals, small-scale transport is enhanced for both small and large $d$ and lower in the center of the LSC around $d=0$. Even though a pronounced large scale organisation evidently exists, this pattern does not lead to a non-zero correlation coefficient due to its reflection symmetry around $d=0$. The horizontal standard deviation $\Delta(\theta w)^{\prime}$ amounts to typically $10 \%$ to $20 \%$ of $\langle(\theta w)\rangle$ (see figure $6.7 \mathrm{i}$ ). These figures are comparable in terms of $N u$ (cf. figure $6.7 \mathrm{~g}$ ), such that spatial variations of the small scale heat transport are indeed significant on a global level.

\section{Conditioned wavenumber}

As a final point in our analysis, we reconsider spatial variations of the local wavenumber defined in (6.7). We normalize the conditioned wavenumber fluctuations $\bar{k}_{\xi}(z, d)$ by their mean $K_{\xi}(z)$ at the respective height to obtain

$$
\kappa_{\xi}(z, d)=\frac{\bar{k}_{\xi}(z, d)}{K_{\xi}(z)} .
$$



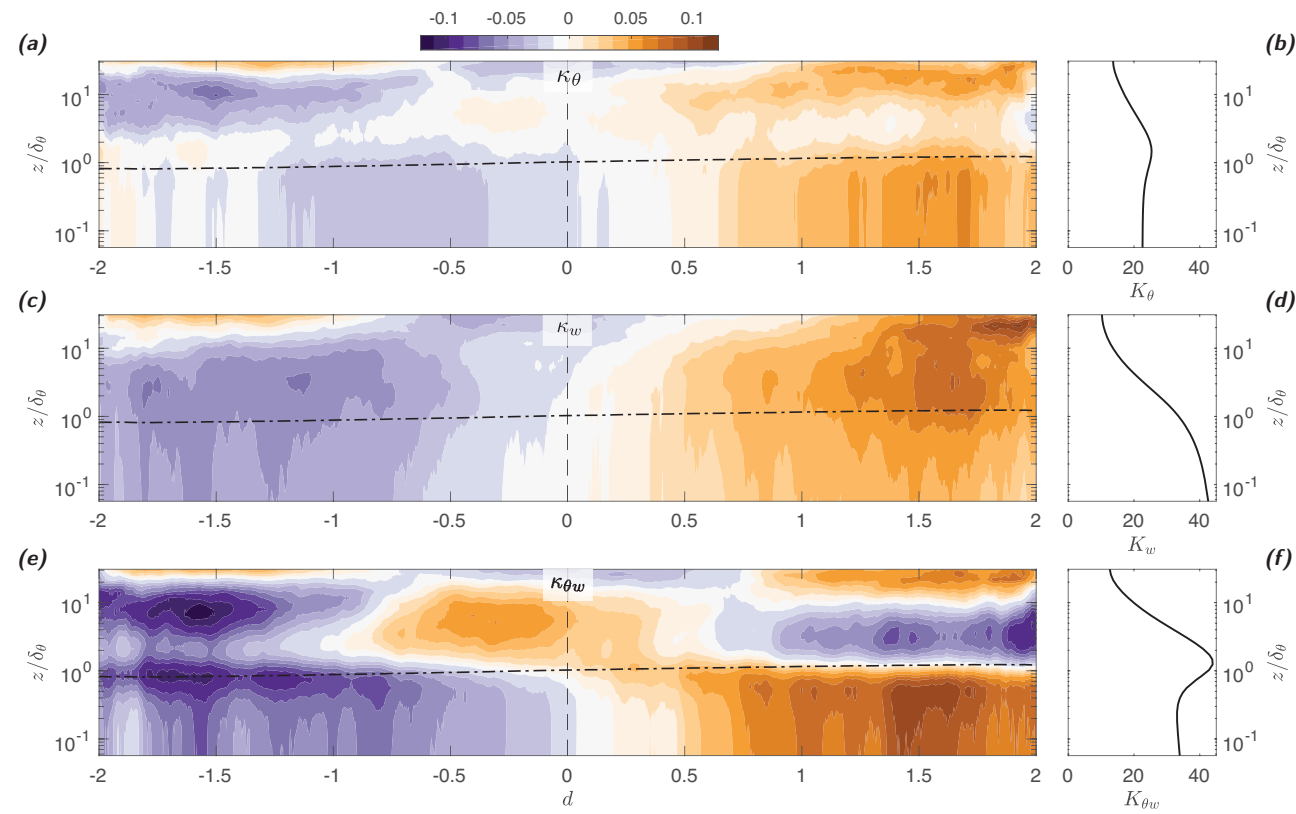

Figure 6.8: Normalized conditioned wavenumber fluctuations of different fields: (a) temperature, (c) wall normal velocity and (e) heat transport. The respective means as a function of $z$ used for normalization are shown in (b,d,f). The colorbar applies to all contour plots.

The results for $\kappa_{\theta}, \kappa_{w}$, and $\kappa_{\theta w}$ are presented in figure $6.8(\mathrm{a}, \mathrm{c}, \mathrm{e})$, respectively, along with the corresponding means $K_{\theta}, K_{w}$, and $K_{\theta w}$ in figure $6.8(\mathrm{~b}, \mathrm{~d}, \mathrm{f})$ for reference.

Largely, the plots of $\kappa_{\theta}, \kappa_{w}$ recount the observations made in figure 6.4(a,b), indicating higher wavenumbers on the plume emitting side for both quantities. This trend appears to be slightly more pronounced for $w$ compared to $\theta$.

The strongest wavenumber modulation is observed for $\kappa_{\theta w}$ in figure $6.8 \mathrm{e}$. The trend there agrees with $\kappa_{\theta}$ and $\kappa_{w}$ (and also with $R_{\theta w, k}$ in figure 6.4c) in indicating higher wavenumbers at $d>0$ approximately up to BL height $\delta_{\theta}$. Similar to $\rho_{\theta w}$, in the bulk a pronounced modulation pattern is also visible for $\kappa_{\theta w}$, which remains hidden in $R_{\theta w, k}$ due to its symmetry. In the case of $\kappa_{\theta w}$, this pattern is reversed for $\delta_{\theta} \lesssim z \lesssim 10 \delta_{\theta}$, for which positive $\kappa_{\theta w}$ is found in the center instead of at large $|d|$. 


\subsection{Concluding remarks}

Our analysis on the basis of correlations as well as through conditioned averaging has revealed significant large-scale organization in small-scale turbulence fields in RBC. These modulation effects were seen to be strongest within - but by no means limited to- a layer close to the wall. Changes in the small-scale organization patterns occurred around a wall height of $z \approx 2 \delta_{\theta}$. This value also marks the start of the 'mixing zone', described by [177]. They find that this mixing zone is characterized by the emergence of intermittent, strong, hot plumes, that lead to long exponential tails in the probability distribution function $(\mathrm{PDF})$ of the temperature. In their case, the mixing region differs from the 'boundary region', where the temperature PDF is described by homogeneous, purely Gaussian, background fluctuations. This distinction does not appear to be relevant in the present context. Further, the crossover height of $z \approx 2 \delta_{\theta}$ links to a sharp decrease in vertical coherence with reference at BL height of the temperature field at large wavenumbers observed by [169]. This is indicative that indeed small structures emanating from the BL do not extend beyond this height, which may explain why the small-scale organisation changes there.

In RBC, the small-scales are typically most energetic close to the wall, making our results most relevant in this region; the strongest correlations are also encountered here with coefficients up to 0.9 for the temperature amplitude. Our findings regarding the near-wall zone are summarized graphically in figure 6.9. Up to a wall distance of $z \approx 2 \delta_{\theta}$, we find that the amplitude of small scale structures (indicted as plumes in figure 6.9) in the plume impacting region is higher, while the wavenumber (and hence the structure size) is higher there. Most importantly, we find that the turbulent heat transport carried by the small-scales is higher (on the order of $10 \%$ of $N u$ ) in the plume impacting region and decreased by the same amount on the emitting side. Significant large-scale organisation of the small scales was also identified in the turbulent bulk of the flow. However, the patterns are more complicated in this case and vary between different quantities, which precludes a straightforward (graphical) summary of the findings. Among the most notable results concerning the bulk flow is the fact that small-scale transport is enhanced at both sides of the LSC and is minimal in the center around $d=0$.

Thus far, we have not touched on the cause of the spatial variations in the small-scale statistics. The vertical localisation of the peak in $\left\langle\theta_{S}^{2}\right\rangle$ correlates with the local maximum in the production term of temperature variance, which 


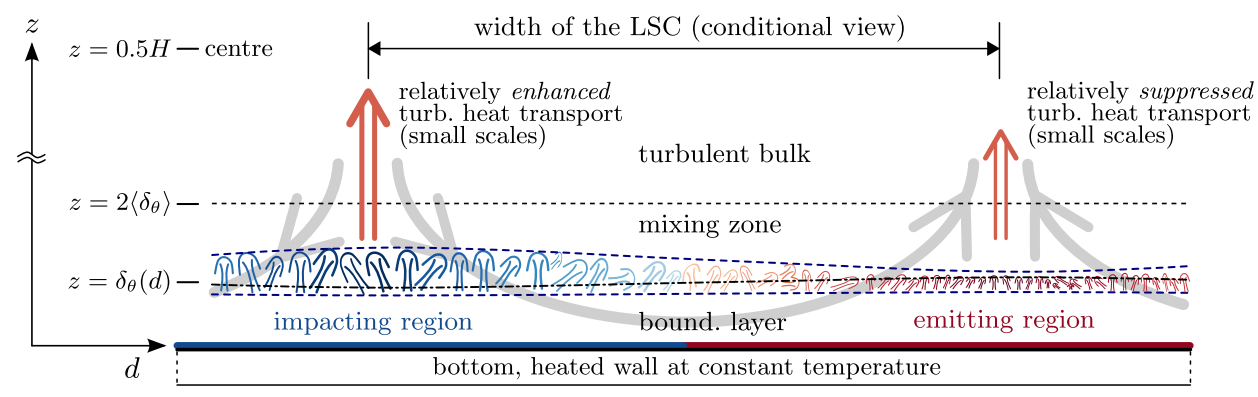

Figure 6.9: Schematic of the large-scale footprint in small-scale near wall RBC turbulence. Small-scale structures are shown as plumes with their height indicating the local magnitude and variations in spacing the local size (i.e. the wavenumber) of the fluctuations.

is proportional to $\theta w \partial_{z} \Theta$ see e.g. [194,195]. It appears therefore likely that also horizontal differences in $\left\langle\theta_{S}^{2}\right\rangle$ in the BL region might arise from locally varying production rates. Indeed, $\overline{N u}$, and hence also the temperature gradient within the $\mathrm{BL}$, is highest for $d<0$, which coincides with the strongest amplitude in $\left\langle\theta_{S}^{2}\right\rangle$. Statistics of $\theta$ and $w$ are interconnected since temperature fluctuations drive production of $\left\langle w^{2}\right\rangle$. Wall-normal velocity fluctuations then feed back to temperature fluctuations since the term $\theta w$ also features in the production term for $\left\langle\theta_{S}^{2}\right\rangle$. This picture is consistent at least with our results for $\rho_{\theta}, \rho_{w}$ as well as for $\rho_{\theta w}$ in the near wall-region. To what extent this theory actually applies will have to be evaluated in future studies. One way to address this would be to study the $R a$ dependence of the effects described here. Recent studies in 2D [56] and 3D RBC [178] indicate that the $\overline{N u}$ distribution changes with increasing $R a$. This should lead to changes in the small-scale patterns if the above is true. A complete answer is likely even more complicated since the different scalings identified in [173] are linked to different leading order balances (inertia-viscous vs. inertia-bouyancy) [196]. Naturally, such a fundamental difference is also to have an impact on the quantities studied here.

A further relevant question is how the strength of the scale interaction varies as a function of $R a$. Experience from flat plate turbulent boundary layers shows that in this case the modulation of small scales by the large superstructure scales intensifies with increasing shear Reynolds number $R e_{\tau}$ e.g. [182]. It remains to be seen if a similar trend exists in RBC. This will also shed light 
on whether a 'universal small-scale signal' exists in pure convection. That is, if the properly normalized statistics of the small-scale signal at a given $z$ are invariant for different locations within the LSC and over at least a limited range of $R a$ once they are stripped of the large-scale footprint by 'demodulation'. Identifying such a universal signal will be crucial in representing small-scale turbulence in RBC effectively. 


\section{Conclusions}

In this thesis we first and foremost investigated the effects of roughness on a turbulent flow with streamwise curvature. We did so by employing numerical simulations, experiments and theoretical analysis of turbulent Taylor-Couette (TC) flow. Following up on previous work in the Physics of Fluids group on regular roughness (like grooves [41] and bars [42]), we started the thesis with simulations of irregular, sand grain, roughness.

In chapter 1, we studied the roughness induced disturbances to the velocity field, and measured the dispersive fluctuations of the azimuthal velocity component, $\tilde{u}=\langle u\rangle_{t}-\langle u\rangle_{\theta, t}$. This dispersive term was obtained by means of a triple decomposition to the azimuthal velocity. We defined the height of the roughness sublayer $h_{r}$ there where the dispersive fluctuations become very small, such that $\sqrt{\left\langle\tilde{u}^{+2}\right\rangle_{\theta, z}}=0.01\left\langle u^{+}\right\rangle_{\theta, z, t}$ and found the height of the roughness sublayer to be $h_{r}=2.78 k_{s}$. This height of the roughness sublayer compares well with values found for other canonical systems [58]. The low height of the roughness sublayer in TC flow, and the existence of similarity of the flow above this sublayer, leads us to believe that we can utilize the shift of the logarithmic region to find an equivalent sand grain roughness height. In chapter 2 we studied smooth wall TC turbulence. We applied the theory of Bradshaw [14], in analogy to that of thermally stratified turbulent boundary layers (BLs) [66], to the curved turbulent BLs of inner cylinder (IC) rotating TC flow. In this analogy, the destabilizing effects from curvature of the streamlines in IC rotating TC flow are similar to the destabilizing effects coming from unstable thermal stratification in the atmospheric BL.

We show that the curvature Obukhov length $L_{c}$ [14] separates the spatial regions that are dominated by shear and curvature effects. We find that for $\delta_{\nu}<y \lesssim 0.20 L_{c}$, with $\delta_{\nu}$ the viscous length scale and $y$ the wall-normal distance, the mean angular velocity profile in the BL is described by the classical shear profile, with the slope given by the von Kármán constant $\kappa^{-1}=0.39^{-1}$. In contrast, for $0.20 L_{c} \lesssim y \lesssim 0.65 L_{c}$, where curvature effects are relevant, the 
slope of the angular velocity profile is $\lambda^{-1}=0.64^{-1}$. For $y \gtrsim 0.65 L_{c}$ curvature effects dominate, and a region with constant angular momentum sets in. This theory is applied to - and found consistent with - PIV measurements and high-fidelity DNS data covering a wide range of radius ratios $0.50 \leq \eta \leq 0.909$ and rotation rates $10^{8} \leq \mathrm{Ta} \leq 10^{12}$, and describes both the IC BL and the OC BL.

Building on these findings we obtain a new functional form of the mean angular velocity profile in TC turbulence, with separate spatial regions where curvature and shear effects are respectively relevant. In following the theory by Cheng et al. [15], and matching our new outer BL profile with the constant angular momentum profile in the bulk at the edge of the BL, we recover their $N u(T a, \eta)$ (and $C_{f}\left(R e_{i}, \eta\right)$ ) relations but with a different constant. For the present smooth-wall flow with the outer cylinder (OC) stationary, this supports their model, with fair agreement with various data sets at high $T a$ and different $\eta$.

In chapter 3 we went back to rough wall TC turbulence, and carried out experiments of IC rotating (and stationary OC) TC turbulence with a dynamically fully rough IC and a smooth OC. We measured the torque, and, by means of PIV, the mean angular velocity profiles.

Using the mean azimuthal velocity profiles, we found that the slope of the fully rough asymptote, characterized by $\kappa=0.34 \pm 0.02$, was similar to previous findings in flat plate BLs $\kappa \approx 0.38$. Also, the value of the equivalent sand grain roughness height $k_{s}$ compared reasonably well with those found for sandpaper in flat plate BLs $[108,110]$.

Finally, to obtain the relationship between the dimensionless torque and dimensionless driving of the system $N u(T a)$, we employed a matching argument between the IC BL rough mean angular momentum profile at the IC BL height, and the smooth OC BL mean angular momentum profile, at the OC BL height, based on the CPS model of [15], see also [97]. To justify this, we first showed that for a rough wall IC, a region of constant angular momentum exists in the bulk. We find a convincing overlap between the calculated value of the torque (or wall shear stress), and the experimentally measured values of the torque, with a maximum error of $\approx 5 \%$.

These findings indicate that the turbulent TC facility can be a valuable setup for characterizing the drag properties of any rough surface. Direct and straightforward measurements of the torque can now be translated to a value of the equivalent sand grain roughness height $k_{s}$. It seems that the value of $k_{s}$ found in TC is similar to the value of $k_{s}$ found in flat plate BLs. 
In chapter 4, we investigated, both numerically and experimentally, large Taylor number TC flow in the presence of spanwise-varying roughness, which consists of an arrangement of stripes of width $\tilde{s}$, that covers the entire circumference of the IC. In the experiments, the stripes were made from sandpaper, while in the numerics a confocal microscopy scan of the surface was implemented by means of the immersed boundary method (IBM).

Remarkably, we have found that by varying $\tilde{s}$ in the range $\tilde{s}=[0.47,3.74]$ we can alter the spanwise wavelength of the turbulent Taylor vortices within the range $\tilde{\lambda} \in[0.94,4.01]$, even if the roughness height was very low $(k / d \approx 0.01)$. This manipulation was observed to hold at $\mathrm{Ta}=\mathcal{O}\left(10^{9}\right)$ and $\mathrm{Ta}=\mathcal{O}\left(10^{12}\right)$.

For increasing $\mathrm{Ta}$, the relative strength of the turbulent Taylor vortices with respect to the mean flow decreases. Therefore, we find a weaker imprint of the vortices on the angular velocity profiles for PIV measurements at $\mathrm{Ta}=$ $O\left(10^{12}\right)$, than we do for the DNSs at Ta $=O\left(10^{9}\right)$, for smooth walls. Consequently, we also find that the effects of spanwise varying roughness on the flow are somewhat stronger at $\mathrm{Ta}=O\left(10^{9}\right)$ than for $\mathrm{Ta}=O\left(10^{12}\right)$.

In chapter 5 we performed direct numerical simulations (DNS) of counterrotating TC flow at $400 \leq R e_{i} \leq 1200$ and $-2000 \leq R e_{o} \leq-1000$. For the aspect ratio $\Gamma$ of the domain $42 \leq \Gamma=L / d \leq 125$ with periodic boundary conditions in the axial direction, and the radius ratio $\eta=r_{i} / r_{o}=0.91$. In this regime we found the coexistence of spatio-temporal intermittent laminar and turbulent domains, commonly referred to as spiral turbulence $[16,141]$.

The formation of the turbulent structure (i.e. spiral in TC) out of a turbulent base flow is similar to the phenomenology of the (Complex) Ginzburg-Landau (GL) equations with a noise term [17], in the limit of very low curvature $(\eta=0.98)$. With these fully resolved simulations, we showed that the GL phenomenology also holds for the spirals when curvature effects do play a role. We found that the GL description for pattern formation holds close to the bifurcation point for both varying $R e_{i}$ and varying $R e_{o}$. This once more suggests the existence of a finite wavelength instability in a fully turbulent flow.

The most unstable wavelength of the instability is found to be $\lambda_{z, c} \approx 41 d$, thereby differing from findings in plane Couette flow and in TC flow, where it is $\lambda_{z, c} \approx 32 d$ at very high $\eta=0.98$. Apparently, $\lambda_{z, c}$ is a function of the radius ratio $\eta$. This finding may be an important clue for further theoretical investigations, and it may point the way towards understanding the modulated turbulent states in terms of a stationary bifurcation.

Finally, in chapter 6 , we revealed on the basis of correlations as well as through 
conditioned averaging significant large-scale organization in small-scale turbulence fields in RBC. These modulation effects were seen to be strongest within - but by no means limited to - a layer close to the wall.

\section{Outlook}

In outlook of future work, we propose that more studies in both turbulent flat plate BLs and turbulent TC flow, with identical rough surfaces, are carried out to further compare the drag properties of these surfaces. We also propose studies of the effects of even more considerable roughness penetrating the curvature affected logarithmic regime of the $\mathrm{BL}$, which is related to finding the slope of the fully rough asymptote in that region. This could be achieved by employing a TC setup with a lower radius ratio $\eta$, thus increasing curvature effects.

The key assumption made by [14], namely that production of turbulent kinetic energy is locally balanced by dissipation, as also employed in this thesis, are left to be addressed by means of DNS at very high Reynolds numbers of $R e=O\left(10^{6}\right)$. Whether the Obukhov (outer) logarithmic region will survive at arbitrary high $R e_{i}$ remains an open question. Open questions also concern the effects of stably stratified TC flow (i.e. OC rotation), or even mixed stratified TC flow (i.e. counter cylinder rotation) within the framework of the Monin-Obukhov similarity theory. However, so far, only velocity profiles with a scale separation up to $R e_{\tau} \approx 1200$ are available for OC rotation [95] to apply the theoretical analysis. 


\section{References}

[1] A. S. Monin and A. M. Yaglom, Statistical Fluid Mechanics, volume 2, MIT Press, Cambridge, Mass. (1975).

[2] L. Prandtl, "Über Flüssigkeitsbewegung bei sehr kleiner Reibung", Verhandl 3, Intern. Math. Kongr. Heidelberg, Auch: Gesammelte Abhandlungen 484-491 (1904).

[3] J. Jiménez, "Cascades in wall-bounded turbulence", Annu. Rev. Fluid Mech. 44, 27 (2012).

[4] A. A. Townsend, The Structure of Turbulent Shear Flow, Cambridge University Press, Cambridge, UK, 1st edition (1956).

[5] D. Chung, N. Hutchins, M. P. Schultz, and K. A. Flack, "Predicting the drag of roughness", Annu. Rev. Fluid Mech. In Press (2021).

[6] J. Jiménez, "Turbulent flows over rough walls", Annu. Rev. Fluid Mech. 36, 173 (2004).

[7] M. Raupach, R. Antonia, and S. Rajagopalan, "Rough-Wall Turbulent Boundary Layers", J. Fluids Eng. 44, 1 (1991).

[8] F. Hama, "Boundary-layer characteristics for smooth and rough surfaces.", Trans. Soc. Nav. Archit. Mar. Eng. 62, 333 (1954).

[9] F. H. Clauser, "Turbulent boundary layers in adverse pressure gradients", J. Aeronaut. Sci. 21, 91 (1954).

[10] J. Nikuradse, "Strömungsgesetze in rauhen Rohren (trans. Flow laws in rough pipes)", Forschungsheft Arb. Ing. Wes. 361 (1933).

[11] S. B. Pope, Turbulent Flow, Cambridge University Press, Cambridge (2000). 
[12] G. I. Taylor, "Stability of a viscous liquid contained between two rotating cylinders", Phil. Trans. R. Soc. A 223, 289 (1923).

[13] S. Grossmann, D. Lohse, and C. Sun, "High Reynolds number TaylorCouette turbulence", Annu. Rev. Fluid Mech. 48, 53 (2016).

[14] P. Bradshaw, "The analogy between streamline curvature and buoyancy in turbulent shear flow", J. Fluid Mech. 36, 177 (1969).

[15] W. Cheng, D. I. Pullin, and R. Samtaney, "Large-Eddy simulation and modeling of Taylor-Couette flow with an outer stationary cylinder", J. Fluid Mech. 890, A17 (2020).

[16] D. Coles, "Transition in circular Couette flow", J. Fluid Mech. 21, 385 (1965).

[17] A. Prigent, G. Grégoire, H. Chaté, O. Dauchot, and W. van Saarloos, "Large-Scale Finite-Wavelength Modulation within Turbulent Shear Flows", Phys. Rev. Lett. 89, 014501 (2002).

[18] A. Scotti, "Direct numerical simulation of turbulent channel flows with boundary roughened with virtual sandpaper", Phys. Fluids 18, 031701 (2006).

[19] P. M. Ligrani and R. J. Moffat, "Structure of transitionally rough and fully rough turbulent boundary layers", J. Fluid Mech. 162, 69 (1986).

[20] M. Schultz and K. Flack, "The rough-wall turbulent boundary layer from the hydraulically smooth to the fully rough regime", J. Fluid Mech. 580, 381 (2007).

[21] L. Chan, M. Macdonald, D. Chung, N. Hutchins, and A. Ooi, "A systematic investigation of roughness height and wavelength in turbulent pipe flow in the transitionally rough regime", J. Fluid Mech. 771, 743 (2015).

[22] A. Busse, M. Thakkar, and N. Sandham, "Reynolds-number dependence of the near-wall flow over irregular rough surfaces", J. Fluid Mech. 810, 196 (2017).

[23] K. A. Flack and M. P. Schultz, "Review of hydraulic roughness scales in the fully rough regime", Trans. ASME: J. Fluids Eng. 132, 041203 (2010). 
[24] F. H. Busse, "Viewpoint: The Twins of Turbulence Research", Physics 5, 4 (2012).

[25] B. Eckhardt, S. Grossmann, and D. Lohse, "Torque scaling in turbulent Taylor-Couette flow between independently rotating cylinders", J. Fluid Mech. 581, 221 (2007).

[26] K. A. Flack and M. P. Schultz, "Roughness effects on wall-bounded turbulent flows", Phys. Fluids 26, 101305 (2014).

[27] S. G. Huisman, S. Scharnowski, C. Cierpka, C. Kähler, D. Lohse, and C. Sun, "Logarithmic boundary layers in strong Taylor-Couette turbulence", Phys. Rev. Lett. 110, 264501 (2013).

[28] H. Schlichting, "Experimentelle Untersuchungen zum Rauigkeitsproblem.", Ing. Arch. 36, 173 (1936).

[29] A. J. Musker, "Universal Roughness Functions for Naturally-Occurring Surfaces", Trans. Can. Soc. Mech. Eng. 1, 1 (1980).

[30] E. Napoli, V. Armenio, and M. D. Marchis, "The effect of the slope of irregularly distributed roughness elements on turbulent wall-bounded flows", J. Fluid Mech. 613, 385 (2008).

[31] M. MacDonald, L. Chan, D. Chung, N. Hutchins, and A. Ooi, "Turbulent flow over transitionally rough surfaces with varying roughness densities", J. Fluid Mech. 804, 130 (2016).

[32] K. A. Flack, M. P. Schultz, and W. B. Rose, "The onset of roughness effects in the transitionally rough regime", Int. J. Heat Fluid Flow 35, 160 (2012).

[33] C. F. Colebrook, "Turbulent flow in pipes, with particular reference to the transitional region between smooth and rough wall laws", J. Inst. Civil Engrs 11, 133 (1939).

[34] M. A. Shockling, J. J. Allen, and A. J. Smits, "Roughness effects in turbulent pipe flow", J. Fluid Mech. 564, 267 (2006).

[35] M. Thakkar, A. Busse, and N. D. Sandham, "Direct numerical simulation of turbulent channel flow over a surrogate for Nikuradse-type roughness", J. Fluid Mech. 837, R1 (2018). 
[36] C. Chan-Braun, M. Garcia-Villalba, and M. Uhlmann, "Force and torque acting on particles in a transitionally rough open-channel flow", J. Fluid Mech. 684, 441 (2011).

[37] L. F. Moody, "Friction factors for pipe flow", Trans. ASME 66, 671 (1944).

[38] P. Bradshaw, "A note on 'critical roughness height' and 'transitional roughness"', Phys. Fluids 12, 1611 (2000).

[39] O. Cadot, Y. Couder, A. Daerr, S. Douady, and A. Tsinober, "Energy injection in closed turbulent flows: Stirring through boundary layers versus inertial stirring", Phys. Rev. E 56, 427 (1997).

[40] T. H. van den Berg, C. R. Doering, D. Lohse, and D. Lathrop, "Smooth and rough boundaries in turbulent Taylor-Couette flow", Phys. Rev. E 68, 036307 (2003).

[41] X. Zhu, R. Ostilla-Mónico, R. Verzicco, and D. Lohse, "Direct numerical simulation of Taylor-Couette flow with grooved walls: torque scaling and flow structure", J. Fluid Mech. 794, 746 (2016).

[42] X. Zhu, R. Verzicco, and D. Lohse, "Disentangling the origins of torque enhancement through wall roughness in Taylor-Couette turbulence", J. Fluid Mech. 812, 279 (2017).

[43] X. Zhu, R. A. Verschoof, D. Bakhuis, S. G. Huisman, R. Verzicco, C. Sun, and D. Lohse, "Wall roughness induces asymptotic ultimate turbulence", Nature Physics 14, 417 (2018).

[44] J. Yuan and U. Piomelli, "Roughness effects on the Reynolds stress budgets in near-wall turbulence", J. Fluid Mech. 760, R1 (2014).

[45] R. Ostilla-Mónico, E. P. van der Poel, R. Verzicco, S. Grossmann, and D. Lohse, "Boundary layer dynamics at the transition between the classical and the ultimate regime of Taylor-Couette flow", Phys. Fluids 26, 015114 (2014).

[46] R. Ostilla-Mónico, R. Verzicco, and D. Lohse, "Effects of the computational domain size on direct numerical simulations of Taylor-Couette turbulence with stationary outer cylinder", Phys. Fluids 27, 025110 (2015). 
[47] H. J. Brauckmann and B. Eckhardt, "Direct numerical simulations of local and global torque in Taylor-Couette flow up to Re $=30$ 000", J. Fluid Mech. 718, 398 (2013).

[48] B. Eckhardt, T. M. Schneider, B. Hof, and J. Westerweel, "Turbulence transition in pipe flow", Annu. Rev. Fluid Mech. 39, 447 (2007).

[49] D. P. Lathrop, J. Fineberg, and H. S. Swinney, "Transition to sheardriven turbulence in Couette-Taylor flow", Phys. Rev. A 46, 6390 (1992).

[50] R. Verzicco and P. Orlandi, "A finite-difference scheme for threedimensional incompressible flow in cylindrical coordinates", J. Comput. Phys. 123, 402 (1996).

[51] E. P. van der Poel, R. Ostilla-Mónico, J. Donners, and R. Verzicco, "A pencil distributed finite difference code for strongly turbulent wallbounded flows", Comput. Fluids 116, 10 (2015).

[52] E. A. Fadlun, R. Verzicco, P. Orlandi, and J. Mohd-Yusof, "Combined Immersed-Boundary Finite-Difference Methods for Three-Dimensional Complex Flow Simulations", J. Comput. Phys. 161, 35 (2000).

[53] G. Iaccarino and R. Verzicco, "Immersed boundary technique for turbulent flow simulations", Appl. Mech. Rev. 56, 331 (2003).

[54] G. Stringano, G. Pascazio, and R. Verzicco, "Turbulent thermal convection over grooved plates", J. Fluid Mech. 557, 307 (2006).

[55] R. Ostilla-Mónico, R. Verzicco, S. Grossmann, and D. Lohse, "The nearwall region of highly turbulent Taylor-Couette flow", J. Fluid Mech. 788, 95 (2015).

[56] X. Zhu, V. Mathai, R. J. A. M. Stevens, R. Verzicco, and D. Lohse, "Transition to the ultimate regime in two-dimensional Rayleigh-Bénard convection", Phys. Rev. Lett. 120, 144502 (2018).

[57] E. P. van der Poel, R. Ostilla-Mónico, R. Verzicco, S. Grossmann, and D. Lohse, "Logarithmic mean temperature profiles and their connection to plume emissions in turbulent Rayleigh-Bénard convection", Phys. Rev. Lett. 115, 154501 (2015). 
[58] D. Pokrajac, J. L. Campbell, V. Nikora, C. Manes, and I. McEwan, "Quadrant analysis of persistent spatial velocity perturbations over square-bar roughness", Exp. Fluids 42, 413 (2007).

[59] D. Chung, J. P. Monty, and A. Ooi, "An idealised assessment of Townsend's outer-layer similarity hypothesis for wall turbulence", J. Fluid Mech. 742, R3 (2014).

[60] J. van der Walt, J. Schönberger, J. Nunez-Iglesias, F. Boulogne, J. Warner, N. Yager, E. Gouillart, T. Yu, and the scikit-image contributors., "scikit-image: Image processing in Python", (2014), PeerJ 2:e453 [Online; accessed December 2017].

[61] A. Sigal and J. E. Danberg, "New correlation of roughness density effect on the turbulent boundary layer.", AIAA J. 28, 554 (1990).

[62] B. N. J. Persson, O. Albohr, U. Tartaglino, A. I. Volokitin, and E. Tosatti, "On the nature of surface roughness with application to contact mechanics, sealing, rubber, friction and adhesion", J. Phys. Condens. Matter 17, R1 (2005).

[63] H. Schlichting, Boundary layer theory, McGraw Hill, New York, 7th edition (1979).

[64] A. J. Smits, B. J. McKeon, and I. Marusic, "High-Reynolds Number Wall Turbulence", Annu. Rev. Fluid Mech. 43, 353 (2011).

[65] A. M. Obukhov, "Turbulence in an atmosphere with a non-uniform temperature", Bound.-Layer Meteorol. 2, 7 (1971).

[66] A. S. Monin and A. M. Obukhov, "Basic laws of turbulent mixing in the surface layer of the atmosphere", Contrib. Geophys. Inst. Acad. Sci. UDSSR 151, 163 (1954).

[67] P. S. Marcus, "Simulation of Taylor-Couette flow. Part 1. Numerical methods and comparison with experiment", J. Fluid Mech. 146, 45 (1984).

[68] W. V. R. Malkus and G. Veronis, "Finite amplitude cellular convection", J. Fluid Mech. 4, 225 (1958).

[69] S. Grossmann and D. Lohse, "Logarithmic temperature profiles in the ultimate regime of thermal convection", Phys. Fluids 24, 125103 (2012). 
[70] D. Krug, X. Yang, C. M. de Silva, R. Ostilla-Mónico, R. Verzicco, I. Marusic, and D. Lohse, "Statistics of turbulence in the energycontaining range of Taylor-Couette compared to canonical wall-bounded flows", J. Fluid Mech. 830, 797 (2017).

[71] D. P. Lathrop, J. Fineberg, and H. S. Swinney, "Turbulent flow between concentric rotating cylinders at large Reynolds numbers", Phys. Rev. Lett. 68, 1515 (1992).

[72] R. H. Kraichnan, "Turbulent thermal convection at arbitrary Prandtl number", Phys. Fluids 5, 1374 (1962).

[73] X. Chavanne, F. Chilla, B. Castaing, B. Hebral, B. Chabaud, and J. Chaussy, "Observation of the ultimate regime in Rayleigh-Bénard convection", Phys. Rev. Lett. 79, 3648 (1997).

[74] S. Grossmann and D. Lohse, "Multiple scaling in the ultimate regime of thermal convection", Phys. Fluids 23, 045108 (2011).

[75] S. G. Huisman, D. P. M. van Gils, S. Grossmann, C. Sun, and D. Lohse, "Ultimate turbulent Taylor-Couette flow", Phys. Rev. Lett. 108, 024501 (2012).

[76] R. C. A. van der Veen, S. G. Huisman, S. Merbold, W. Harlander, C. Egbers, D. Lohse, and C. Sun, "Taylor-Couette turbulence at radius ratio $\eta=0.5$ : scaling, flow structures and plumes", J. Fluid Mech. 799, $334(2016)$.

[77] S. Grossmann, D. Lohse, and C. Sun, "Velocity profiles in strongly turbulent Taylor-Couette flow", Phys. Fluids 26, 025114 (2014).

[78] Lord Rayleigh, "On the dynamics of revolving fluids.", Proc. R. Soc. London Ser. A 93 (1916).

[79] L. D. Landau and E. M. Lifshitz, Fluid Mechanics, Pergamon Press, Oxford (1987).

[80] D. P. M. van Gils, G. W. Bruggert, D. P. Lathrop, C. Sun, and D. Lohse, "The Twente turbulent Taylor-Couette $\left(T^{3} C\right)$ facility: strongly turbulent (multi-phase) flow between independently rotating cylinders", Rev. Sci. Instrum. 82, 025105 (2011). 
[81] S. Merbold, H. J. Brauckmann, and C. Egbers, "Torque measurements and numerical determination in differentially rotating wide gap TaylorCouette flow", Phys. Rev. E 87, 023014 (2013).

[82] S. Grossmann and D. Lohse, "Curvature effects on the velocity profile in turbulent pipe flow", Eur. Phys. J. E Soft Matter 40, 16 (2017).

[83] R. D. Moser and P. Moin, "Direct Numerical Simulation of Curved Turbulent Channel Flow", NASA Ames Center Tech. Memo. (1984) 85974.

[84] A. A. Townsend, The Structure of Turbulent Shear Flow, Cambridge University Press, Cambridge, UK, 2nd edition (1976).

[85] A. Esser and S. Grossmann, "Analytic expression for Taylor-Couette stability boundary", Phys. Fluids 8, 1814 (1996).

[86] I. Marusic, B. J. McKeon, P. A. Monkewitz, H. M. Nagib, A. J. Smits, and K. R. Sreenivasan, "Wall-bounded turbulent flows at high Reynolds numbers: Recent advances and key issues", Phys. Fluids 22, 065103 (2010).

[87] J. P. Johnston, R. M. Halleent, and D. K. Lezius, "Effects of spanwise rotation on the structure of two-dimensional fully developed turbulent channel flow", J. Fluid Mech. 56, 533 (1972).

[88] X. Yang, Z. Xia, J. Lee, Y. Lv, and J. Yuan, "Mean flow scaling in a spanwise rotating channel", Center for Turbulence Research Annual Research Briefs 285-297 (2018).

[89] F. Wendt, "Turbulente Strömungen zwischen zwei rotierenden Zylindern", Ingenieurs-Archiv 4, 577 (1933).

[90] H. J. Brauckmann, M. Salewski, and B. Eckhardt, "Momentum transport in Taylor-Couette flow with vanishing curvature", J. Fluid Mech. 790, 419 (2016).

[91] K. Nakabayashi and O. Kitoh, "Low Reynolds number fully developed two-dimensional turbulent channel flow with system rotation", J. Fluid Mech. 315, 1 (1996).

[92] A. Froitzheim, S. Merbold, O. R., and C. Egbers, "Angular momentum transport and flow organization in Taylor-Couette flow at radius ratio of $\eta=0.357 "$, Phys. Rev. Fluids 4, 084605 (2019). 
[93] R. Ostilla-Mónico, E. P. van der Poel, R. Verzicco, S. Grossmann, and D. Lohse, "Exploring the phase diagram of fully turbulent TaylorCouette flow", J. Fluid Mech. 761, 1 (2014).

[94] D. P. M. van Gils, S. G. Huisman, G. W. Bruggert, C. Sun, and D. Lohse, "Torque scaling in turbulent Taylor-Couette flow with co- and counterrotating cylinders", Phys. Rev. Lett. 106, 024502 (2011).

[95] R. Ostilla-Mónico, R. Verzicco, and D. Lohse, "Turbulent TaylorCouette flow with stationary inner cylinder", J. Fluid Mech. 799, R1 (2016).

[96] P. Berghout, X. Zhu, D. Chung, R. Verzicco, R. Stevens, and D. Lohse, "Direct numerical simulations of Taylor-Couette turbulence: the effects of sand grain roughness", J. Fluid Mech. 873, 260 (2019).

[97] P. Berghout, R. Verzicco, R. J. A. M. Stevens, D. Lohse, and D. Chung, "Calculation of the mean velocity profile for strongly turbulent TaylorCouette flow and arbitrary radius ratios", (2020).

[98] P. Forooghi, A. Stroh, F. Magagnato, S. Jakirlić, and B. Frohnapfel, "Toward a Universal Roughness Correlation", J. Fluids Eng. 139, 121201 (2017).

[99] G. I. Taylor, "Experiments on the motion of solid bodies in rotating fluids", Proc. R. Soc. London A 104, 213 (1923).

[100] D. P. M. van Gils, S. G. Huisman, S. Grossmann, C. Sun, and D. Lohse, "Optimal Taylor-Couette turbulence", J. Fluid Mech. 706, 118 (2012).

[101] S. G. Huisman, R. C. A. van der Veen, C. Sun, and D. Lohse, "Multiple states in highly turbulent Taylor-Couette flow", Nature Commun. 5, 3820 (2014).

[102] R. A. Verschoof, X. Zhu, D. Bakhuis, S. G. Huisman, R. Verzicco, C. Sun, and D. Lohse, "Rough-wall turbulent Taylor-Couette flow: The effect of the rib height", Eur. Phys. J. E. 41, 125 (2018).

[103] D. Bakhuis, R. Ezeta, P. Berghout, P. Bullee, N. Tai, D. Chung, R. Verzicco, D. Lohse, S. Huisman, and C. Sun, "Controlling secondary flow in Taylor-Couette turbulence through spanwise-varying roughness", J. Fluid Mech. 883, A15 (2020). 
[104] W. J. Baars, D. T. Squire, K. M. Talluru, M. R. Abbassi, N. Hutchins, and I. Marusic, "Wall-drag measurements of smooth- and rough-wall turbulent boundary layers using a floating element", Exp. Fluids 57 (2016).

[105] C. J. Kähler, U. Scholz, and J. Ortmanns, "Wall-shear-stress and nearwall turbulence measurements up to single pixel resolution by means of long-distance micro-PIV", Exp. Fluids 41, 327 (2006).

[106] C. J. Kähler, S. Scharnowski, and C. Cierpka, "On the resolution limit of digital particle image velocimetry", Exp. Fluids 52, 1629 (2012).

[107] S. Scharnowski, R. Hain, and C. J. Kähler, "Reynolds stress estimation up to single-pixel resolution using PIV-measurements", Exp. Fluids 52, 985 (2012).

[108] D. T. Squire, C. Morrill-Winter, N. Hutchins, M. P. Schultz, J. C. Klewicki, and I. Marusic, "Comparison of turbulent boundary layers over smooth and rough surfaces up to high Reynolds numbers", J. Fluid Mech. 795, 210 (2016).

[109] K. Flack, M. Schultz, and B. J.M., "Skin Friction Measurements of Systematically-Varied Roughness: Probing the Role of Roughness Amplitude and Skewness", Flow Turb. Combust. 104, 317 (2020).

[110] K. A. Flack, M. P. Schultz, and J. S. Connelly, "Examination of a critical roughness height for outer layer similarity", Phys. Fluids 19, 095104 (2007).

[111] A. Tilgner, A. Belmonte, and A. Libchaber, "Temperature and velocity profiles of turbulence convection in water", Phys. Rev. E 47, R2253 (1993).

[112] G. Ahlers, S. Grossmann, and D. Lohse, "Heat transfer and large scale dynamics in turbulent Rayleigh-Bénard convection", Rev. Mod. Phys. 81, 503 (2009).

[113] H. Ren and Y. Wu, "Turbulent boundary layers over smooth and rough forward-facing steps", Phys. Fluids 23, 045102 (2011).

[114] K. Koeltzsch, A. Dinkelacker, and R. Grundmann, "Flow over convergent and divergent wall riblets", Exp. Fluids 33, 346 (2002). 
[115] W. Anderson, J. M. Barros, K. T. Christensen, and A. Awasthi, "Numerical and experimental study of mechanisms responsible for turbulent secondary flows in boundary layer flows over spanwise heterogeneous roughness", J. Fluid Mech. 768, 316 (2015).

[116] D. Chung, J. P. Monty, and N. Hutchins, "Similarity and structure of wall turbulence with lateral wall shear stress variations", J. Fluid Mech. 847, 591 (2018).

[117] Z. Q. Wang and N. S. Cheng, "Time-mean structure of secondary flows in open channel with longitudinal bedforms", Adv. Water Resour. 29, 1634 (2006).

[118] J. O. Hinze, "Secondary Currents in Wall Turbulence", Phys. Fluids 10, S122 (1967).

[119] J. O. Hinze, "Experimental investigation on secondary currents in the turbulent flow through a straight conduit", Appl. Sci. Res. 28, 453 (1973).

[120] B. Nugroho, N. Hutchins, and J. Monty, "Large-scale spanwise periodicity in a turbulent boundary layer induced by highly ordered and directional surface roughness", Int. J. Heat Fluid Fl. 41, 90 (2013).

[121] J. M. Barros and K. T. Christensen, "Observations of turbulent secondary flows in a rough-wall boundary layer", J. Fluid Mech. 748, R1 (2014).

[122] R. Mejia-Alvarez and K. T. Christensen, "Wall-parallel stereo particleimage velocimetry measurements in the roughness sublayer of turbulent flow overlying highly irregular roughness", Phys. Fluids 25, 115109 (2013).

[123] D. Willingham, W. Anderson, K. T. Christensen, and J. M. Barros, "Turbulent boundary layer flow over transverse aerodynamic roughness transitions: Induced mixing and flow characterization", Phys. Fluids 26, 025111 (2014).

[124] C. Vanderwel and B. Ganapathisubramani, "Effects of spanwise spacing on large-scale secondary flows in rough-wall turbulent boundary layers", J. Fluid Mech. 774, R2 (2015). 
[125] J. Yang and W. Anderson, "Numerical study of turbulent channel flow over surfaces with variable spanwise heterogeneities: Topographicallydriven secondary flows affect outer-layer similarity of turbulent length scales", Flow Turbul. Combust. 100, 1 (2017).

[126] P. Bradshaw, "Turbulent Secondary Flows", Annu. Rev. Fluid Mech. 19, 53 (1987).

[127] T. Medjnoun, C. Vanderwel, and B. Ganapathisubramani, "Characteristics of turbulent boundary layers over smooth surfaces with spanwise heterogeneities", J. Fluid Mech. 838, 516 (2018).

[128] A. Chouippe, E. Climent, D. Legendre, and C. Gabillet, "Numerical simulation of bubble dispersion in turbulent Taylor-Couette flow", Phys. Fluids 26, 043304 (2014).

[129] R. C. A. van der Veen, S. G. Huisman, O. Y. Dung, H. L. Tang, C. Sun, and D. Lohse, "Exploring the phase space of multiple states in highly turbulent Taylor-Couette flow", Phys. Rev. Fluids 1, 024401 (2016).

[130] M. Gul, G. E. Elsinga, and J. Westerweel, "Experimental investigation of torque hysteresis behaviour of Taylor-Couette Flow", J. Fluid Mech. 836, 635 (2017).

[131] R. Ostilla-Mónico, S. G. Huisman, T. J. G. Jannink, D. P. M. V. Gils, R. Verzicco, S. Grossmann, C. Sun, and D. Lohse, "Optimal TaylorCouette flow: radius ratio dependence", J. Fluid Mech. 747, 1 (2014).

[132] G. Stringano and R. Verzicco, "Mean flow structure in thermal convection in a cylindrical cell of aspect-ratio one half", J. Fluid Mech. 548, 1 (2006).

[133] S. G. Huisman, D. P. M. van Gils, and C. Sun, "Applying Laser Doppler Anemometry inside a Taylor-Couette geometry using a ray-tracer to correct for curvature effects", European J. Mech. B/Fluids 36, 115 (2012).

[134] M. Wilczek, H. Xu, and Y. Narita, "A note on Taylor's hypothesis under large-scale flow variation", Nonlin. Processes Geophys. 21, 645 (2014).

[135] M. S. Paoletti and D. P. Lathrop, "Angular Momentum Transport in Turbulent Flow between Independently Rotating Cylinders", Phys. Rev. Lett. 106, 024501 (2011). 
[136] D. Bakhuis, R. Ostilla Mónico, E. P. van der Poel, R. Verzicco, and D. Lohse, "Mixed insulating and conducting thermal boundary conditions in Rayleigh-Bénard convection", J. Fluid Mech. 835, 491 (2018).

[137] J. Rolland and P. Manneville, "Ginzburg-Landau description of laminarturbulent oblique band formation in transitional plane Couette flow", Eur. Phys. J. B 80, 529 (2011).

[138] C. D. Andereck, S. S. Liu, and H. L. Swinney, "Flow regimes in a circular Couette system with independently rotating cylinders", J. Fluid Mech. 164, 155 (1986).

[139] D. Barkley, "Theoretical perspective on the route to turbulence in a pipe", J. Fluid Mech. 643, 495 (2016).

[140] D. Barkley and L. S. Tuckerman, "Computational study of turbulent laminar patterns in Couette flow", Phys. Rev. Lett. 94, 014502 (2005).

[141] C. Van Atta, "Exploratory measurements in spiral turbulence", J. Fluid Mech. 25, 495 (1966).

[142] R. P. Feynman, "Lecture Notes in Physics", Addison-Wesley, Reading, MA 2 (1964).

[143] J. J. Hegseth, C. D. Andereck, F. Hayot, and Y. Pomeau, "Spiral Turbulence and Phase Dynamics", Phys. Rev. Lett. 62, 257 (1989).

[144] A. Meseguer, F. Mellibovsky, M. Avila, and F. Marques, "Instability mechanisms and transition scenarios of spiral turbulence in TaylorCouette flow", Phys. Rev. E 80, 046315 (2009).

[145] K. Coughlin and P. S. Marcus, "Turbulent Bursts in Couette-Taylor Flow", Phys. Rev. Lett. 77, 2214 (1996).

[146] C. F. Hamill, "Turbulent Bursting in the Couette-Taylor System", MA Thesis, Univ. Texas at Austin (1995).

[147] S. Dong, "Evidence for internal structures of spiral turbulence", Phys. Rev. E 80, 067301 (2009).

[148] S. Dong and X. Zheng, "Direct numerical simulation of spiral turbulence", J. Fluid Mech. 668, 150 (2011). 
[149] M. J. Burin and C. J. Czarnocki, "Subcritical transition and spiral turbulence in circular Couette flow", J. Fluid Mech. 709, 106 (2012).

[150] D. Barkley and L. S. Tuckerman, "Mean flow of turbulent-laminar patterns in plane Couette flow", J. Fluid Mech. 576, 109 (2007).

[151] L. S. Tuckerman and D. Barkley, "Patterns and dynamics in transitionial plane Couette flow", Phys. Fluids 23, 041301 (2011).

[152] W. van Saarloos, "The complex Ginzburg-Landau equation for beginners", in: P.E. Cladis, P. PalDy-Muhoray , Proceedings of the Santa Fe Workshop on 'Spatio-Temporal Patterns in Nonequilibrium Complex Systems'. Addison-Wesley, Chicago (1994).

[153] M. Cross and H. Greenside, Pattern formation and dynamics in nonequilibrium systems, Cambridge University Press, Cambridge, UK (2009).

[154] A. Prigent, G. Grégoire, H. Chaté, and O. Dauchot, "Long-wavelength modulation of turbulent shear flows", Physica D: Nonlinear Phenomena 174, 100 (2003).

[155] A. Goharzadeh and I. Mutabazi, "Measurement of coefficients of the Ginzburg-Landau equation for patterns of Taylor spirals", Phys. Rev. E 82, 016306 (2010).

[156] R. Ostilla-Mónico, R. J. A. M. Stevens, S. Grossman, R. Verzicco, and D. Lohse, "Optimal Taylor-Couette flow: direct numerical simulations", J. Fluid Mech. 719, 14 (2013).

[157] A. Prigent and O. Dauchot, "Barber pole turbulence in large aspect ratio Taylor-Couette flow", arXiv:cond-mat/00009241 (2000).

[158] D. L. Hartmann, L. A. Moy, and Q. Fu, "Tropical convection and the energy balance at the top of the atmosphere", J. Climate 14, 4495 (2001).

[159] F. Cattaneo, T. Emonet, and N. Weiss, "On the interaction between convection and magnetic fields", Astrophys. J. 588, 1183 (2003).

[160] E. D. Siggia, "High Rayleigh number convection", Annu. Rev. Fluid Mech. 26, 137 (1994).

[161] D. Lohse and K. Q. Xia, "Small-scale properties of turbulent RayleighBénard convection", Annu. Rev. Fluid Mech. 42, 335 (2010). 
[162] F. Chilla and J. Schumacher, "New perspectives in turbulent RayleighBénard convection", Eur. Phys. J. E 35, 58 (2012).

[163] D. E. Fitzjarrald, "An experimental study of turbulent convection in air", J. Fluid Mech. 73, 693 (1976).

[164] T. Hartlep, A. Tilgner, and F. H. Busse, "Large Scale Structures in Rayleigh-Bénard Convection at High Rayleigh Numbers", Phys. Rev. Lett. 91, 064501 (2003).

[165] A. Parodi, J. von Hardenberg, G. Passoni, A. Provenzale, and E. A. Spiegel, "Clustering of plumes in turbulent convection", Phys. Rev. Lett. 92, 194503 (2004).

[166] J. von Hardenberg, A. Parodi, G. Passoni, A. Provenzale, and E. A. Spiegel, "Large-scale patterns in Rayleigh-Bénard convection", Physics Letters A 372, 2223 (2008).

[167] A. Pandey, J. D. Scheel, and J. Schumacher, "Turbulent superstructures in Rayleigh-Bénard convection", Nature Commun. 9, 2118 (2018).

[168] R. J. A. M. Stevens, A. Blass, X. Zhu, R. Verzicco, and D. Lohse, "Turbulent thermal superstructures in Rayleigh-Bénard convection", Phys. Rev. Fluids 3, 041501(R) (2018).

[169] D. Krug, D. Lohse, and R. J. A. M. Stevens, "Coherence of temperature and velocity superstructures in turbulent Rayleigh-Bénard flow", J. Fluid Mech. 887, A2 (2020).

[170] N. Hutchins and I. Marusic, "Evidence of very long meandering features in the logarithmic region of turbulent boundary layers", J. Fluid Mech. 579, 1 (2007).

[171] M. Lee and R. D. Moser, "Extreme-scale motions in turbulent plane Couette flows", J. of Fluid Mech. 842, 128 (2018).

[172] A. Fonda, E. Pandey, J. Schumacher, and K. Sreenivasan, "Deep learning in turbulent convection networks", Proc. Natl. Acad. Sci. 116, 8667 (2019).

[173] Y. H. He and K. Q. Xia, "Temperature Fluctuation Profiles in Turbulent Thermal Convection: A Logarithmic Dependence versus a Power-Law Dependence", Phys. Rev. Lett. 122, 014503 (2019). 
[174] O. Shishkina, S. Horn, S. Wagner, and E. S. C. Ching, "Thermal boundary layer equation for turbulent Rayleigh-Bénard convection", Phys. Rev. Lett. 114, 114302 (2015).

[175] Y. Wang, X. He, and P. Tong, "Boundary layer fluctuations and their effects on mean and variance temperature profiles in turbulent RayleighBénard convection", Phys. Rev. Fluids 1, 082301 (2016).

[176] Y. Wang, W. Xu, X. He, H. Yik, X. Wang, J. Schumacher, and P. Tong, "Boundary layer fluctuations in turbulent Rayleigh-Bénard convection", J. Fluid Mech. 840, 408 (2018).

[177] Y. Wang, X. He, and P. Tong, "Turbulent temperature fluctuations in a closed Rayleigh-Bénard convection cell”, J. Fluid Mech. 874, 263 (2019).

[178] A. Blass, R. Verzicco, D. Lohse, R. J. A. M. Stevens, and D. Krug, "Flow organization in laterally unconfined Rayleigh-Bénard turbulence", arXiv preprint arXiv:2006.01505 (2020).

[179] G. L. Brown and A. S. W. Thomas, "Large structure in a turbulent boundary layer", Phys. Fluids 10, S243 (1977).

[180] P. R. Bandyopadhyay and A. K. M. F. Hussain, "The coupling between scales in shear flows", Phys. Fluids 27, 2221 (1984).

[181] N. Hutchins and I. Marusic, "Large-scale influences in near-wall turbulence", Phil. Trans. R. Soc. A 365, 647 (2007).

[182] R. Mathis, N. Hutchins, and I. Marusic, "Large-scale amplitude modulation of the small-scale structures in turbulent boundary layers", J. Fluid Mech. 628, 311 (2009).

[183] I. Marusic, R. Mathis, and N. Hutchins, "Predictive model for wallbounded turbulent flows", Science 329, 193 (2010).

[184] D. Chung and B. J. McKeon, "Large-eddy simulation of large-scale structures in long channel flow", J. Fluid Mech. 661, 341 (2009).

[185] P. Schlatter and R. Örlü, "Quantifying the interaction between large and small scales in wall-bounded turbulent flows: a note of caution", Phys. Fluids 22 (2010), 051704. 
[186] M. Bernardini and S. Pirozzoli, "Inner/outer layer interactions in turbulent boundary layers: a refined measure for the large-scale amplitude modulation mechanism", Phys. Fluids 23 (2011), 061701.

[187] B. Ganapathisubramani, N. Hutchins, J. P. Monty, D. Chung, and I. Marusic, "Amplitude and frequency modulation in wall turbulence", J. Fluid Mech. 712, 61 (2012).

[188] W. J. Baars, N. Hutchins, and I. Marusic, "Reynolds number trend of hierarchies and scale interactions in turbulent boundary layers", Phil. Trans. R. Soc. A 375, 20160077 (2017).

[189] E. Dogan, R. Örlü, D. Gatti, R. Vinuesa, and P. Schlatter, "Quantification of amplitude modulation in wall-bounded turbulence", Fluid Dyn. Res. 51 (2019), 011408.

[190] W. J. Baars, K. M. Talluru, N. Hutchins, and I. Marusic, "Wavelet analysis of wall turbulence to study large-scale modulation of small scales", Exp. Fluids 56, 188 (2015).

[191] M. Farge, "Wavelet Transforms and Their Application to Turbulence", Annu. Rev. Fluid Mech. 24, 395 (1992).

[192] P. S. Addison, The illustrated wavelet transform handbook, Taylor \& Francis, New York (2002).

[193] S. Wagner, O. Shishkina, and C. Wagner, "Boundary layers and wind in cylindrical Rayleigh-Bénard cells", J. Fluid Mech. 697, 336 (2012).

[194] J. Deardorff and G. Willis, "Investigation of turbulent thermal convection between horizontal plates", J. Fluid Mech. 28, 675 (1967).

[195] A. Togni, R. Cimarelli and E. De Angelis, "Physical and scale-byscale analysis of Rayleigh-Bénard convection.", J. Fluid Mech. 782, 380 (2015).

[196] R. J. Adrian, "Variation of temperature and velocity fluctuations in turbulent thermal convection over horizontal surfaces", Int. J. Heat Mass Transf. 39, 2303 (1996). 


\section{Summary}

In this thesis we have studied the effects of streamwise curvature on a rough and smooth wall-bounded turbulent flow. To this end, we have studied a canonical system of fluid mechanics, Taylor-Couette (TC) flow. TC flow is the flow in between two independently rotating, concentric, cylinders. In chapter 1, we studied direct numerical simulations (DNSs) of TC turbulence with sand grain roughness mounted on the inner cylinder. We focus on the effects of the roughness height $k_{s}^{+}$in the transitionally rough regime. We find that the downwards shift of the logarithmic layer, due to transitionally rough sand grains exhibits remarkably similar behavior to that of the Nikuradse (VDIForschungsheft 361, 1933) data of sand grain roughness in pipe flow, regardless of the Taylor number dependent constants of the logarithmic layer. Furthermore, we find that the dynamical effects of the sand grains are contained to the roughness sublayer $h_{r}$ with $h_{r}=2.78 k_{s}$.

In recent years, high-fidelity simulations and experiments revealed the shape of the streamwise and angular velocity profiles in turbulent TC flow up to very high Reynolds numbers. However, due to curvature effects, so far no theory has been able to correctly describe the turbulent streamwise velocity profile for given radius ratio, as the classical Prandtl-von Kármán logarithmic law for turbulent boundary layers over a flat surface at most fits in a limited spatial region. In chapter 2 we address this deficiency by applying the idea of a Monin-Obukhov curvature length to turbulent TC flow. This length separates the flow regions where the production of turbulent kinetic energy is governed by pure shear from that where it acts in combination with the curvature of the streamlines. We demonstrate that for all Reynolds numbers and radius ratios, the mean streamwise and angular velocity profiles collapse according to this separation. We then develop the functional form of the velocity profile. Finally, using the newly developed angular velocity profiles, we show that these lead to an alternative contant in the model proposed by [15] for the dependence of the torque on the Reynolds number, or, in other words, of the 
generalized Nusselt number (i.e., the dimensionless angular velocity transport) on the Taylor number.

Mapping any given roughness geometry to its fluid dynamic behaviour has been hampered by the lack of accurate and direct measurements of skin-friction drag. Here the TC system provides an opportunity as it is a closed system and allows to directly and reliably measure the skin-friction. However, the wall-curvature potentially complicates the connection between the wall friction and the wall roughness characteristics. In chapter 3 we investigate the effects of a hydrodynamically fully rough surface on highly turbulent, inner cylinder rotating, TC flow. We find that the effects of a hydrodynamically fully rough surface on TC turbulence, where the roughness height $k$ is three orders of magnitude smaller than the Obukhov curvature length $L_{c}$ [97], are similar to those effects of a fully rough surface on a flat plate turbulent boundary layer (BL). Hence, the value of the equivalent sand grain height $k_{s}$, that characterizes the drag properties of a rough surface, is similar to those found for comparable sandpaper surfaces in a flat plate BL. Next, we obtain the dependence of the torque (skin-friction drag) on the Reynolds number for given wall roughness, and find agreement with the experimental results within $5 \%$. Our findings demonstrate that global torque measurements in the TC facility are well suited to reliably deduce wall drag properties for any rough surface. Chapter 4 deals with 'unusual' roughness. Unusual, non-homogeneous, roughness means in this context that the roughness contains a characteristic length scale that is not smaller than the gap width, but is of the same order. This leads to the invalidation of a classic modelling assumptions (outer layer similarity), and consequently adds majorly to the complexity of the problem.

In chapter 5 we shift the focus again to Taylor-Couette flow with smooth walls, and study the emergence and dynamics of very large structures at the onset of turbulence (or better, the onset of laminarization). These structures, already observed by D. Coles (1965) [16], manifest themselves as large turbulent spirals that slowly disappear when the cylinders rotate slower and slower. Prigent et al. (2000) [17] found that the formation of these spirals fits the phenomenology of the Complex Ginzburg-Landau model and this suggests that they are a finite wavelength instability. In our simulations, we show that this description holds when curvature effects do play a role.

Finally, we study Rayleigh-Bénard flow, the flow between a heated and cooled plate, in chapter 6 . In particular we focus on the frequency and amplitude modulation of small-scale turbulence by the large-scale convection role. 


\section{Samenvatting}

In dit proefschrift hebben we de effecten onderzocht van stromingsgewijze kromming op een ruwe en gladde, door een wand begrensde, turbulente stroming. Om deze effecten te onderzoeken, hebben we een van de canonieke systemen van de vloeistofmechanica, Taylor-Couette (TC) stroming, bestudeerd. TC-stroming is de stroming tussen twee onafhankelijk roterende, concentrische cilinders. In hoofdstuk 1 hebben we directe numerieke simulaties (DNS) van TC-turbulentie bestudeerd met zandkorrelruwheid gemonteerd op de binnencilinder. We richten ons op de invloed van de ruwheidshoogte $k_{s}^{+}$in het transitioneel ruwe regime. We vinden dat de neerwaartse verschuiving van de logaritmische laag, als gevolg van overgangsruwe zandkorrels, opmerkelijk vergelijkbaar gedrag vertoont met gegevens van zandkorrelruwheid in buisstroom van de Nikuradse (VDI-Forschungsheft 361, 1933), ongeacht de Taylornummerafhankelijke constanten van de logaritmische laag. Verder vinden we dat de dynamische effecten van de zandkorrels zijn vervat in de ruwheidssublaag $h_{r}$ met $h_{r}=2,78 k_{s}$.

In de afgelopen jaren hebben simulaties en experimenten de vorm van de stromingsgewijze en hoeksnelheid snelheidsprofielen in turbulente TC stroming tot zeer hoge Reynoldsgetallen onthuld. Echter, als gevolg van krommingseffecten is tot dusverre geen theorie in staat geweest om het turbulente stromingsgewijze snelheidsprofiel voor een gegeven radiusverhouding correct te beschrijven, aangezien de klassieke Prandtl-von Kármán logaritmische wet voor turbulente grenslagen over een vlak oppervlak hooguit van toepassing is in een beperkt ruimtelijk gebied dicht bij de gekromde wand. In hoofdstuk 2 pakken we deze tekortkoming aan door het idee van een Monin-Obukhov kromminglengte toe te passen op turbulente TC-stroming. Deze lengte scheidt de stroomgebieden waar de productie van turbulente kinetische energie wordt beheerst door pure afschuiving van die waar die werkt in combinatie met de kromming van de stroomlijnen. We demonstreren dat voor alle Reynoldsgetallen en radiusverhoudingen de gemiddelde hoeksnelheidsprofielen samenvalling vol- 
gens deze scheiding. Vervolgens ontwikkelen we de functionele vorm van het snelheidsprofiel. Ten slotte laten we met behulp van de nieuw ontwikkelde hoeksnelheidsprofielen zien dat deze leiden tot een alternatieve constante in het door [15] voorgestelde model voor de afhankelijkheid van het koppel van het Reynoldsgetal, of, met andere woorden, van het gegeneraliseerde Nusselt getal (dwz het dimensieloze hoeksnelheidstransport) op het Taylor-getal.

Het in kaart brengen van het verband tussen een bepaalde ruwheidsgeometrie en hydrodynamische wrijving word belemmerd door het ontbreken van nauwkeurige en directe metingen van de wrijvingsweerstand. Hier biedt het TC-systeem een kans omdat het een gesloten systeem is en het mogelijk maakt om de wrijving direct en betrouwbaar te meten. De wandkromming bemoeilijkt echter mogelijk de verbinding tussen de wandwrijving en de wandruwheidskarakteristieken. In hoofdstuk 3 onderzoeken we de effecten van een hydrodynamisch volledig ruw oppervlak op zeer turbulente, binnencilinder roterende TC stroming. We vinden dat de effecten van een hydrodynamisch volledig ruw oppervlak op TC-turbulentie, waarbij de ruwheidshoogte $k$ drie orden van grootte kleiner is dan de Obukhov-krommingslengte $L_{c}$ [97], vergelijkbaar zijn met die van een volledig ruw oppervlak op een vlakke plaat turbulente grenslaag. Daarom is de waarde van de equivalente zandkorrelhoogte $k_{s}$, die de weerstandseigenschappen van een ruw oppervlak kenmerkt, vergelijkbaar met die gevonden voor vergelijkbare schuurpapieroppervlakken in een vlakke plaat turbulente grenslaag. Vervolgens verkrijgen we de afhankelijkheid van het koppel (wrijvingsweerstand) van het Reynoldsgetal voor gegeven wandruwheid, en vinden we overeenstemming met de experimentele resultaten binnen $5 \%$. Onze bevindingen tonen aan dat koppelmetingen in de TC-faciliteit zeer geschikt zijn om op betrouwbare wijze wandweerstandseigenschappen af te leiden voor elk ruw oppervlak.

Hoofdstuk 4 gaat over 'ongebruikelijke' ruwheid. Ongebruikelijke, niet-homogene ruwheid betekent in deze context dat de ruwheid een karakteristieke lengteschaal bevat die niet kleiner is dan de afstand tussen de cylinders, maar van dezelfde orde is. Dit leidt tot de ongeldigverklaring van klassieke modelaannames (gelijkenis van de buitenste laag) en draagt bijgevolg in belangrijke mate bij aan de complexiteit van het probleem.

In hoofdstuk 5 verleggen we de focus weer naar TC stroming met gladde wanden, en bestuderen we het ontstaan en de dynamiek van zeer grote structuren bij het begin van turbulentie (of beter, het begin van laminarisering). Deze structuren, reeds waargenomen door D. Coles (1965) [16], manifesteren zich als grote turbulente spiralen die langzaam verdwijnen wanneer de cylin- 
ders langzamer en langzamer draaien. Prigent et al. (2000) [17] ontdekte dat de vorming van deze spiralen past bij de fenomenologie van het Complex Ginzburg-Landau-model en dit suggereert dat ze een eindige golflengteinstabiliteit zijn. In onze simulaties laten we zien dat deze beschrijving ook opgaat als krommingseffecten wel een rol spelen.

Ten slotte bestuderen we Rayleigh-Bénard stroming, de stroming tussen een verwarmde en gekoelde plaat, in hoofdstuk 6 . In het bijzonder richten we ons op de frequentie- en amplitudemodulatie van kleinschalige turbulentie door de grootschalige convectierol. 


\section{Acknowledgements}

Now the thesis comes to an end, it is time to thank those that helped me directly, or indirectly, in finishing this thesis. It certainly was not a oneman job. Also, I want to thank those people that did not contribute to this thesis, perhaps even did the opposite, but made my time the last four years so enjoyable.

Dear Detlef, I want to thank you first of all for letting me carry out this $\mathrm{PhD}$ in your group. I highly valued the freedom and unlimited resources you gave me in finding my own way. Meanwhile, you always found time to answer my questions, to discuss my results, and to provide me with plenty of feedback on the manuscripts. There are few better places for a young researcher, if there are any, than the group that you built and lead. Thank you.

Daniel, thank you for your immeasurable contribution to this thesis. It was a humbling experience, but great pleasure, to work with you on four papers. I am still amazed by the seemingly never ending stream of input that you provided. In particular, I remember the fruitful meetings in Turin, Madrid, Enschede and Melbourne. Thank you also for all discussions on unrelated matters which I have thoroughly enjoyed. I hope we will meet again soon.

Roberto and Richard, thank you for your help throughout. Richard, your calm and sincere supervision was always very much appreciated. I admire your fine eye for detail and strong push for code and data organization. I believe that any student in the numerics group strongly benefits from your presence. Roberto, you are the cornerstone of the numerics group. I want to thank you for the many answers on questions relating mainly the numerical procedures, but also the flow physics. You also frequently provided us during meetings with amusing anecdotes or extra motivation if need be. Thank you for all.

Dear Wim, I want to thank you for the very enjoyable collaboration we had on spiral turbulence. In particular I value the generous extra explanation you gave me, on a topic that was new to me, during meetings in Leiden and 
Amsterdam. Your kind and patient supervision meant a lot. Thank you.

Dear Harry, you supervised me when I started my academic research, during the two years when we worked on Lattice Boltzmann simulations in Ireland. Although I moved on to work on turbulence, I have enjoyed those years tremendously, and I have profited with every new paper from the many lessons you taught me. Thank you. I hope to visit Limerick again soon.

Dominik, you joined the group somewhat later but managed to teach me plenty. Your high standards in general, and your knowledge of spectral analysis of turbulent flows in particular, kept me motivated in the final year of the $\mathrm{PhD}$. More important, I hope your tendons and ligaments live long enough to finally let me beat you in beach or/and show you how to properly surf. See you soon. Sander, as head of the experimental Taylor-Couette group we frequently met. I have gotten to know you as a sharp and positive mind. You did not shy away from helping the experimentalists with the experimental work and analysis. I want to thank you for the fruitful collaborations, and helpful comments on the manuscripts. I also want to thank the rest of the TC experimental group that I collaborated with. Dennis, Rodrigo, Pim and Yuan, thank you all for the excellent work. I have learned a lot from you. Also, Dennis van Gils, thank you for assisting continuously and for providing me with your data on numerous requests. Joanita, thank you for all the help on the organizing side. Bas, thank you for your help with anything related to electronics. I had the pleasure to work with Jacco, Alvaro and Michiel in teaching Dynamics and Relativity. Thanks Jacco for your inspiring attitude, I really think students can not wish for a better teacher. Of course, I also have to thank you for our meeting in Brussels, that brought me to PoF. Alvaro, it was a pleasure to work with you too. Michiel, I very much enjoyed our trips to Nantahala and Seattle, like I enjoyed our frequent discussions about cycling and more. Be sure to visit Delft soon!

I have been very lucky with my office mates in the legendary ME24/7. Jelle, it was a great pleasure to share an office with you. We often discussed our individual projects, start-up ideas, Ron Swanson, etc. It still hurts that our collaboration on drag reduction is put on halt due to the pandemic, but I am hopeful that those measurements will someday be carried out. I want to thank you for the nice trips to Nantahala, Turin, Oktoberfest, for teaching me how to juggle, and being a very solid football player! UJ, legend, it was a blessing to be your office mate and housemate. Your ventures into Peaky Blinders, Bitcoins, Paul Theroux books and so much more will never be forgotten. I was as often stunned as I was in tears of laughter by your tweets and general 
lifestyle. Hank Moody would be proud, I am absolutely sure. Srinidhi, you were a very kind and relaxed office mate. Thanks for your elaborate lectures on Indian culture and, less frequent, wind parks. I hope we all meet again soon.

Within the research group, I was part of the turbulent numerics subgroup. Upon my arrival in February 2017, the group had a modest size of 5, but grew to more than 3 times that. I owe all of you a word of thanks for the many interactions. Xiaojue, thank you for your close supervision in my first year. Your input accelerated my research tremendously. I also enjoyed our casual interactions on Chinese culture and other things. Thanks for all your help. Vamsi, we have interacted a lot, on many topics. I admire your broad knowledge and interests and your high level coding skills. Thanks for all your help throughout, and the in particular the trips to Tata Steel Chess, Nantahala and Rome. Alex, we were part of the same generation, which gives a special bond. It was always good to visit your office and to discuss our shared hurdles. I want to thank you for your help on all kinds of matters and of course the great trip we had to Maryland with Martin. Also, thanks for continuously saving PoF United from crushing defeats!! CS, thanks for being a calm and extraordinary friendly colleague. I admire your hard-working character and strong ethics. Yantao, thanks for your help at the start. Robert, thanks for taking over the CPU hour and data storage accounting tasks. Steven, thanks for all the discussions. Qi, thanks for all your help with the proposals. All the best in your undoubtedly successful career. Yibo, thanks for being a very kind housemate. Hi Peter, in the first year we were interacted a lot. We lived together and often had dinner together in the Waaier. I want to thank you for all our interactions. You are a wise man, with strong ethics, and many books. I admire your hard work. All the best.

I owe a word of thanks to the 3 students that I supervised. Rick, you carried out both your BSc. and MSc. thesis with me. I want to thank you for your trust in me, your hard work and your positive mindset. Laura, you visited our group for only 3.5 moths. However, the amount of work you managed to do was truly amazing. Timothy, our collaboration was interrupted by the pandemic, and we had to decide to give you another project. Good luck with your $\mathrm{PhD}$ and do not forget those drag reducing bubbles!

Luckily, I have spent my time wisely over the last few years, and only worked when there was no sports to play. I want to thank all those team members that I played with. Those of Brakcelona in the evening competition. Those of PoF United during the lunch competition. In particular I would like to thanks 
Ruben for leading the team before I did. Thanks to all that played beach with me, in particular Maaike and Bram, I hope we get to play some games more. Thanks to all that joined in the Batavierenrace, in particular Martin and Maaike who helped in organizing. Thanks Ruben, for the adventure in the Rocky Mountains. Thanks Pim, for our sailing trips. One day I will make a 'plannetje' and visit you there in Norway.

I would also like to thank my friends from Ireland. Corne, I still miss our hiking and surfing, which we did so often. I also miss the entrepreneurial atmosphere that made it such a great time. Lucas, you definitely brought a tremendous amount of fun with you. The fact that we continue to visit Ireland ever since, tells the story of how much fun it was (and is). Sid, there is no one like you, who continues to give me so many interesting insights on physics and literature. Maulik, Shaurya, Thomas, thanks for your friendship. May we soon reunite for an old school session.

In the category of people that have certainly not contributed to the $\mathrm{PhD}$, or my well-being in general, there are the idiots who call themselves the KdK. Gijs, we met already in high school and have been friends ever since. We studied together, discussed everything together and have done endless stupid things together. Thanks for the laughs and everlasting memories. Patrick, we also met in high school. Same accounts to you, endless days and nights of being stupid. I am happy you only contributed to one broken bone and some broken teeth. Thanks for all. Schipper, I always thought of you as the smartest of us all. But then you married. Still, I hope to see you many more times.

Dan, tot slot, wil ik mijn familie bedanken. Het is een groot voorrecht om op te groeien in, en een onbreekbare band te houden met, ons gezin. Het doet mij goed terug in de buurt te zijn, al betekent dat wel dat ik mijn dekking vaker hoog moet houden. Leen, jij bent de volgende reden voor een feestje, nog even doorbikkelen en je bent er. Ma, ik bewonder je kracht. Ik zou je voor zoveel moeten bedanken, maar het volstaat hier om te zeggen dat ik zonder jou geen brugklas had gehaald, dan weten wij genoeg. Bedankt voor alles.

Dan als allerlaatste, mijn Sunshine. Ik kan het geluk niet bevatten dat ik jou heb gevonden. Zeker weten doe ik niets, behalve dat ik altijd van jou zal houden. Tevens heb ik ook nog eens het geluk dat je een hele leuke familie met je meebrengt. 

